Portland State University

PDXScholar

Summer 8-13-2013

\title{
Fostering Strengths in Incarcerated Youth: The \\ Development of a Measure of Psychological \\ Empowerment in Oregon Youth Authority \\ Correctional Facilities
}

Lindsey Brianna Patterson

Portland State University

Follow this and additional works at: https://pdxscholar.library.pdx.edu/open_access_etds Let us know how access to this document benefits you.

\section{Recommended Citation}

Patterson, Lindsey Brianna, "Fostering Strengths in Incarcerated Youth: The Development of a Measure of Psychological Empowerment in Oregon Youth Authority Correctional Facilities" (2013). Dissertations and Theses. Paper 1086.

https://doi.org/10.15760/etd.1086

This Dissertation is brought to you for free and open access. It has been accepted for inclusion in Dissertations and Theses by an authorized administrator of PDXScholar. Please contact us if we can make this document more accessible: pdxscholar@pdx.edu. 
Fostering Strengths in Incarcerated Youth: The Development of a Measure of Psychological Empowerment in Oregon Youth Authority Correctional Facilities

by

Lindsey Brianna Patterson

A dissertation submitted in partial fulfillment of the requirements for the degree of

Doctor of Philosophy

in

Applied Psychology

Dissertation Committee:

Keith Kaufman, Chair

Todd Bodner

Kris Henning

Lauren Lichty

Shannon Myrick

Portland State University

2013 


\begin{abstract}
Research on juvenile offender treatment and intervention has called for a shift from a deficits-based to a strengths-based approach (Marshall, Ward, Mann, Moulden, Fernandez, Serran, \& Marshall, 2005; Wormith, Althouse, Simpson, Reitzel, Fagan, \& Morgan, 2007; Zeldin, 2004). One potential approach to treatment fosters a sense of psychological empowerment in youth. Although research has yet to explore the experience of psychological empowerment within incarcerated youth, theory on empowerment suggests that it could help youth to create both cognitive (e.g., increased self-esteem, increased confidence) and behavioral (e.g., improving quality of life, social integration) changes in their lives (Cargo, Grams, Ottoson, Ward, \& Green, 2003; Holden, Crankshaw, Nimsch, Hinnant, \& Hund, 2004a). Empowerment-based programming may also help youth develop specific psychosocial capacities, such as competence, confidence, and self-efficacy, which are necessary skills for future success and community reintegration. The purpose of the current study was to establish a measure of psychological empowerment (PE) and explore potential behavioral correlates of PE for young men within Oregon Youth Authority (OYA) correctional and re-entry facilities. Using a cross-sectional, non-experimental design, quantitative data from selfreport surveys of incarcerated youth on PE in three settings within correctional facilities as well as OYA staff ratings of behavioral success in five skill areas was collected. Confirmatory factor analyses did not support the three-factor structure of PE. A singlefactor structure of Intrapersonal PE was found to fit the data in three correctional settings. The present study has implications for the reconceptualization and reoperationalization of psychological empowerment in this unique context. Using the confirmed sub-scale,
\end{abstract}


results of hierarchical linear models indicated that Intrapersonal PE was a significant predictor of behavioral success in two of the five OYA domains. Even with an imperfect operationalization of $\mathrm{PE}$, there was partial evidence for the predictive ability of Intrapersonal PE. 
Acknowledgements

This dissertation is a product of much iteration of ideas and designs. Input from many different perspectives has helped me to finalize this research, and my thanks go to everyone that has contributed to this process.

First, I would like to express my appreciation for my dissertation committee chair and advisor, Dr. Keith Kaufman. Through our work, I have gained invaluable experiences and opportunities for which I am grateful. I also thank Dr. Kaufman for his constant encouragement to combine my research interests in Community Psychology (CP) with the work of his lab. It is with his support that I was able to develop a study that uniquely combines $\mathrm{CP}$ and a youth correctional context.

My community partner, the Oregon Youth Authority, and Dr. Shannon Myrick in particular, were also essential to the completion of my dissertation. Dr. Myrick was eager and enthusiastic about my ideas and was instrumental in implementing the research. On my dissertation committee, I very much appreciate Dr. Myrick's help in critically reflecting on the ways in which the results can be interpreted within OYA. I would also like to thank other dissertation committee members, including Dr. Todd Bodner, Dr. Lauren Lichty, and Dr. Kris Henning, who each contributed to this process in important ways. Dr. Bodner's statistical expertise was essential during the development and analysis phases of this dissertation. Without his guidance, I would not have arrived at the level of confidence I have for decisions made during analysis. Dr. Lichty has provided much insight into empowerment and encouraged me to think about this research through different lenses. With a background in Criminal Justice, Dr. Henning has brought an interesting perspective to this work, situating my dissertation 
within a larger juvenile justice context. Without the involvement of all five of these committee members, this dissertation would not have been possible.

Many thanks go to the youth at the Oregon Youth Authority for the time and effort they contributed to this research. In particular, I would like to express my gratitude to the Youth Advisory Committee whose feedback was informative and encouraging. The participation of youth and staff at OYA has made this study possible.

I also have an incredible amount of gratitude for my family and friends, who have supported me in every way throughout graduate school. I am most grateful for endless support from my partner, Ben Decherd, who has helped me celebrate my successes and overcome struggles. His positive outlook and considerate demeanor has made it easier to balance graduate school and "real life." My parents, Diane and Bill Patterson, have also been instrumental in this process. Thank you both for cultivating my desire to achieve high goals throughout my education. I would also like to thank to my Mom's partner, Vicki Shinneman, who has kept me healthy and energized with plenty of cold, stress, and anxiety remedies. My thanks also go to my grandparents, Don and Mary Dobson and Robin Nichols, as well as my brother, Kellen Patterson, for always lending an ear and cheering for me loud and clear. A big thank you goes to Nancy and Jon Decherd, who have consistently stocked me with "goodies for graduate school" that have brightened my days over the last five years. Finally, I am fortunate to be part of an amazing network of friends and colleagues. I thank you all for exchanging knowledge, insights, and emotional support with me over the years. Without the encouragement of my family and friends graduate school would not have been possible. 
Table of Contents

$\begin{array}{ll}\text { Abstract } & \text { i }\end{array}$

$\begin{array}{ll}\text { Acknowledgements } & \text { iii }\end{array}$

List of Tables $\quad$ ix

List of Figures $\quad$ xi

Chapter I: Introduction and Overview 1

$\begin{array}{ll}\text { Chapter II: The Juvenile Justice System } & 10\end{array}$

$\begin{array}{lr}\text { Introduction and Brief History } & 10\end{array}$

$\begin{array}{ll}\text { Developmental Challenges During Incarceration } & 14\end{array}$

Theory and Research on the Strengths-Based Programming 16 for Incarcerated Youth

$\begin{array}{ll}\text { Chapter III: Empowerment } & 18\end{array}$

$\begin{array}{ll}\text { Introduction } & 18\end{array}$

Importance of Empowerment During Adolescence 22

$\begin{array}{ll}\text { Youth Empowerment Theories } & 27\end{array}$

$\begin{array}{lr}\text { Research on Youth Empowerment } & 28\end{array}$

$\begin{array}{ll}\text { Conclusion } & 33\end{array}$

Chapter IV: Psychological Empowerment Measure Development 36

Assessing Empowerment in Research 36

The Development of a Measure of Psychological Empowerment for 41 Incarcerated Youth

$\begin{array}{ll}\text { Conclusion } & 54\end{array}$

Chapter V: Study Context $\quad 56$

Oregon Youth Authority $\quad 56$ 
Distinctions between OYA and the General Juvenile Justice System

Empowerment with Incarcerated Youth 63

Empowerment, Positive Youth Development, and the 66 Juvenile Justice System

Empowerment and Youth at OYA 69

$\begin{array}{ll}\text { Selection of OYA Settings } & 72\end{array}$

$\begin{array}{ll}\text { Conclusion } & 76\end{array}$

Chapter VI: Development of Research Hypotheses 78

$\begin{array}{ll}\text { The Purpose of the Present Study } & 79\end{array}$

$\begin{array}{ll}\text { Research Hypotheses } & 79\end{array}$

Chapter VII: Methods $\quad 83$

$\begin{array}{ll}\text { Institutional Review Board } & 83\end{array}$

$\begin{array}{lr}\text { Study Context } & 83\end{array}$

$\begin{array}{ll}\text { Participants } & 84\end{array}$

$\begin{array}{lr}\text { Participant Recruitment } & 86\end{array}$

$\begin{array}{ll}\text { Procedure } & 87\end{array}$

$\begin{array}{ll}\text { Design } & 88\end{array}$

$\begin{array}{ll}\text { Quantitative Measures } & 89\end{array}$

Chapter VIII: Results 95

$\begin{array}{ll}\text { Data Screening } & 95\end{array}$

Confirmatory Factor Analyses on the Correlated Three-Factor 98

Model 
Confirmatory Analyses on the Factor Structure of Intrapersonal PE 102

$\begin{array}{ll}\text { Reliability Assessment of Intrapersonal PE } & 108\end{array}$

$\begin{array}{ll}\text { Validity of Intrapersonal PE } & 109\end{array}$

Confirmatory Analyses on the Factor Structure of Interactional PE 111

Confirmatory Analyses on the Factor Structure of Behavioral PE 112

$\begin{array}{ll}\text { Validity of Behavioral PE } & 116\end{array}$

$\begin{array}{ll}\text { Predicting Behavioral Success from Intrapersonal PE } & 116\end{array}$

$\begin{array}{ll}\text { Chapter IX: Discussion } & 121\end{array}$

Confirmatory Factor Analysis on the Correlated Three-factor

$\begin{array}{ll}\text { Psychological Empowerment Model } & 122\end{array}$

Confirmatory Analyses on the Factor Structure of Intrapersonal PE 123

$\begin{array}{ll}\text { Reliability of Intrapersonal PE } & 126\end{array}$

$\begin{array}{ll}\text { Validity of Intrapersonal PE } & 127\end{array}$

Confirmatory Analyses on the Factor Structure of Interactional PE 129

Confirmatory Analyses on the Factor Structure of Behavioral PE 131

$\begin{array}{ll}\text { Validity of Behavioral PE } & 134\end{array}$

Predicting Behavioral success from Intrapersonal PE 135

Predicting Behavioral Success from Intrapersonal PE 135

$\begin{array}{ll}\text { Limitations of Current Study } & 137\end{array}$

Limitations Related to the Factor Structure of Psychological 137

Empowerment

Limitations Related to Construct Validity of Psychological Empowerment

Limitations Related to Predictions of Behavioral Success 
Strengths, Implications, and Future Research Directions 150

$\begin{array}{ll}\text { Tables } & 164\end{array}$

$\begin{array}{ll}\text { Figures } & 199\end{array}$

$\begin{array}{ll}\text { References } & 207\end{array}$

$\begin{array}{ll}\text { Appendices } & 228\end{array}$

Appendix A: OYA Annual Survey 227

Appendix B: Treatment Manager Survey on Behavioral Success in Five Domains 
List of Tables

Table 1: Selected Items and Abbreviations

Table 2: OYA Facility Background

Table 3: Demographic Information for the Sample of Incarcerated Youth

Table 4: Facility Sub-sample Sizes

Table 5: OYA Annual Survey Scale Reliabilities

Table 6: Intrapersonal Psychological Empowerment Inter-Item Correlations

Table 7: Interactional Psychological Empowerment Inter-Item Correlations

Table 8: Behavioral Psychological Empowerment Inter-Item Correlations

Table 9: Estimated Latent Factor Correlations and Estimated Disturbance

Table 11: Standardized Factor Loadings and Estimated Standardized Measurement Error Variances for Intrapersonal and Behavioral PE in all Settings

Table 12: Fit Indices for Intrapersonal and Behavioral PE in all Settings

Table 13: Estimated Inter-item Correlations and Standardized Residual

Table 15: Pairwise Comparisons of Mean Intrapersonal PE Scores

Table 16: Fit Indices Assessing Measurement Invariance for Intrapersonal $P E$ in all Settings

Table 18: Intrapersonal PE and HPES Sub-scale Correlations in the Living Unit 
Table 19: OYA Annual Survey Scale Correlations in Treatment 191

Table 20: Intrapersonal PE and HPES Sub-scale Correlations in Treatment 192

Table 21: OYA Annual Survey Scale Correlations in School or Vocation 193

Table 22: Intrapersonal PE and HPES Sub-scale Correlations in School or 194 Vocation

Table 23: Estimated Inter-item Correlations and Standardized Residual 195

Covariance Matrices for Behavioral PE in all Settings

Table 24: Results of HLM Predicting Behavioral success from Intrapersonal PE 
List of Figures

$\begin{array}{ll}\text { Figure 1: OYA Process Model } & 199\end{array}$

Figure 2: Model of the Theory of Change at OYA 200

Figure 3: Three-factor Model of Components of Psychological 201

Empowerment for Confirmatory Factor Analysis

Figure 4: Theoretical Model Predicting Behavioral success of 202

Psychological Empowerment

Figure 5: Single-factor Model for Intrapersonal PE for Initial

Confirmatory Factor Analysis in Living Unit

Figure 6: Final Single-factor Model for Intrapersonal PE 204

Figure 7: Single-factor Model for Behavioral PE for Initial 205

Confirmatory Factor Analysis in Living Unit

Figure 8: Final Single-factor Model for Behavioral PE 206 


\section{CHAPTER I}

\section{Introduction and Overview}

In 2007, nearly 100,000 youth were incarcerated in the United States (Office of Juvenile Justice and Delinquency Prevention, 2011). Of these, over 60,500 were youth residing in juvenile public facilities (Office of Juvenile Justice and Delinquency Prevention, 2011). Although these statistics are alarming, they may not be completely representative of the total number of individuals under federal or state juvenile jurisdiction because these data are based strictly on incarcerated youth under the age of 21. While approximately $5 \%$ of juveniles reside within adult prisons (Austin, Johnson, \& Gregoriou, 2000), many juvenile public facilities house youth until their mid-twenties. For example, the Oregon Youth Authority (OYA) currently houses nearly 800 youth, 375 of which were sentenced as adults through the Department of Corrections (DOC; Oregon Youth Authority, 2011b). The large number of U.S. adolescents and young adults ${ }^{1}$ incarcerated within the juvenile justice system warrants an examination of the treatment received while under the jurisdiction of State government.

There is no standard treatment for youth within the juvenile justice system because laws and policies for the treatment of juveniles vary by crime and by state. Despite the lack of standards, treatment and intervention programs addressing a number of criminal behaviors typically focus on decreasing the problem behavior in order to prevent recidivism. It is widely accepted that the focus of prevention, intervention, and treatment for juveniles deficits-based, emphasizing the prevention, reduction, or

\footnotetext{
${ }^{1}$ Since the age of incarcerated youth in the juvenile justice system ranges from early adolescence to early adulthood, "youth" will refer to all individuals under the age of 25 who are incarcerated within youth correctional or re-entry facilities. For more discussion about the use of the term "youth," refer to Butts and Travis (2002).
} 
elimination of criminal behavior (Corcoran, 1997; Hunter, Gilbertson, Vedros, \& Morton, 2004; Marshall, Ward, Mann, Moulden, Fernandez, Serran, \& Marshall, 2005; Moore \& Glei, 1995; Ward \& Stewart, 2003; Zeldin, 2004) rather than addressing other factors (e.g., poverty, poor supervision) that may contribute to the direct causes of their incarceration. These programs are intended to reduce crimes committed by youth and keep the general community safe.

Recidivism rates for a variety of crimes remain high, despite the focus on reducing recidivism through treatment focused on problematic or criminal behaviors. At present, there is no national standard for measuring and comparing recidivism across crimes. That said, it is reported that between $23 \%$ and $30 \%$ of OYA youth recidivate (Oregon Youth Authority, 2011b). It is also estimated that approximately $12 \%$ of DOC youth residing in OYA facilities recidivate (Oregon Youth Authority, 2011b). These figures are consistent with national prison data, where approximately $25 \%$ of youth committing a crime at ages 16 or 17 re-offend by the time they are 19 years of age (Snyder \& Sickmund, 2006).

Given high recidivism rates of youth, it is important to examine treatment programming that youth receive while under State custody. Treatment programming has the potential to address criminal behavior and to provide youth with skills for successful community reintegration. As previously mentioned, treatment programs for juvenile offenders most often focus on negative aspects of the individual, only addressing issues related to the offenses (Corcoran, 1997; Hunter et al., 2004; Marshall et al., 2005). Although it is important to understand individual reasons for offending and identify ways to prevent future crimes, programs that emphasize reduction of offending behavior often 
omit efforts to promote positive aspects of the individual (Marshall et al., 2005). Deficits-based intervention and treatment programs can fail to highlight the potential for positive change, do not typically encourage optimism, and do not usually identify or build upon individual's strengths (Marshall et al., 2005; Wormith, Althouse, Simpson, Reitzel, Fagan, \& Morgan, 2007). This is not to say that all treatment programming focused on the reduction of recidivism or problem behavior is detrimental to youth wellbeing. For example, Andrews and Bonta's (1990) classification system for rehabilitation (i.e., the Risk Needs Responsivity or RNR model) assesses youths' criminological risks and needs and provides appropriately intensive treatment aimed at reducing recidivism based on the initial assessment. In addition to youths' risks and needs, their model also stresses the requirement for treatment staff responsivity and the ability to tailor programming to youth's learning styles and personal characteristics, allowing for appropriate fit between the individual and the treatment approach (Andrews \& Bonta, 1990). As Andrews and his colleagues (2011) argue, well-being can be enhanced by creating costs for criminal behavior and rewards for pro-social values. Although they have reported a respectable rate of success (29\%; Andrews \& Bonta, 2007), their model lacks an explicit emphasis on building upon youth strengths in order to fully address youth development in addition to criminological needs. In combination with risks and needs associated with criminal behavior, programming within correctional centers should help youth learn how to succeed in a variety of life domains (e.g., work, school, social life) once they re-enter their community. This combination of skills may help situate youth in a better position to avoid recidivating. 
A number of practitioners and interventionists working with offenders have called for research on new and innovative treatment methods. They have advocated for programs that help offenders better reintegrate into the community through an emphasis on skill development, positivity, optimism, and building a good life (Andres-Hyman, Forrester, Achara-Abrahams, Lauricella, \& Rowe, 2007; Bazemore \& Erbe, 2003; Bazemore \& Terry, 1997; Butts, Mayer, \& Ruth, 2005; Marshall et al, 2005; Wormith et al., 2007). Programs that focus on these aspects of offender treatment may evoke a sense of psychological empowerment.

The experience of psychological empowerment may be beneficial for incarcerated youth. Psychological empowerment, or one's ability to take control of his life, often includes aspects of self-efficacy, competence, mastery, and autonomy (Rappaport, 1981; Zimmerman, 1995). As discussed in later sections, research has indicated that youth empowerment can result in both positive cognitive (e.g., increased self-esteem, increased confidence) and behavioral (e.g., improving quality of life, social integration) changes (Cargo et al., 2003; Holden, Crankshaw, Nimsch, Hinnant, \& Hund, 2004a). Although there is a paucity of research on psychological empowerment among incarcerated youth, this may be a critical strength to cultivate before youth re-enter the community. The transition to community living is often a difficult one, met with a number of barriers to success (Altschuler \& Brash, 2004). Prior to release, youth need to have developed specific psychosocial capacities, such as competence, confidence, and the ability to make change in their own lives. Some leaders in the field of juvenile justice have begun designing such programs, which are implicitly and explicitly aimed at evoking aspects of psychological empowerment. 
Strengths-based programming may be uniquely suited to cultivating a sense of empowerment in incarcerated youth. There is little doubt that treatment models primarily focused on the reduction of problem-behavior, such as the RNR model (Andrews \& Bonta, 1990; Andrews, Bonta, \& Wormith, 2011), can result in positive outcomes that go beyond the prevention of recidivism (e.g., problem-solving skills, self-management). At the same time, strengths-based approaches to treatment might compliment these models by focusing on the general well-being of youth and possibly eliciting aspects of psychological empowerment (Wilson \& Yates, 2009). For example, the "Good Lives Model" (GLM) for the treatment of sexual offenders takes a strengths-based perspective to develop skills necessary to envision and enact a better life (Ward \& Stewart, 2003). Within this treatment approach, juveniles learn to identify and secure primary goods, or intrinsically beneficial actions, experiences, or states of mind, on their own and in their own way (Ward \& Stewart, 2003). In contrast to popular problem-focused treatment programming, the GLM is a relatively new theoretical framework and does not yet have sufficient empirical support, particularly related to the development of empowerment. Theoretically, however, GLM allows for a degree of self-determination in selecting and achieving their pro-social goals.

The effectiveness of a different strengths-based model that aims to empower youth, Multisystemic Therapy (MST), has been supported by a number of studies within the research literature. MST takes a systemic approach to treatment and addresses individual strengths and weaknesses across multiple life domains (e.g., family, school, peer relations). Working with juvenile offenders and their families within communitybased and home settings, MST has been found to reduce recidivism significantly more 
than parent training, individual therapy, and treatment as usual programming (Curtis, Ronan, Borduin, 2004; Timmons-Mitchell, Bender, Kishna, \& Mitchell, 2006). A major goal within MST is for youth to empower themselves to address difficulties on their own through capacity and relationship building (Henggeler, Cunningham, Pickrel, Schoenwalk, \& Brondino, 1996). While research has yet to investigate the degree to which youth participating in MST experience empowerment, it seems to offer promise for reducing offender recidivism.

The GLM and MST are two of the more common approaches to offender treatment that were designed to cultivate individuals' strengths, including aspects of psychological empowerment. Other examples of strengths-based programming exist and can be further studied in order to understand psychological empowerment in a youth incarceration context. While all programming does not fall under an overarching therapeutric approach, the Oregon Youth Authority (OYA) attempts to increase positive youth development through treatment and other activities. Services offered at OYA for incarcerated youth attempt to provide an opportunity for youth to gain a variety of life skills needed to succeed once back in the community. In addition to typical treatment programs that address risk reduction and risk management, these programs include the development of coping and social skills as well as educational and vocational skills (Oregon Youth Authority, 2012b). Within these programs, youth are offered the opportunity to take high school and college courses, work with supervisors within and outside of the facilities to complete a number of projects or jobs, and continue treatment programming focused on life skills and the satisfaction of human needs (Oregon Youth Authority, 2012b). 
There are several ways in which these programs can provide youth the opportunity to experience empowerment. Activities that allow youth the autonomy to learn about and make decisions related to their treatment programming, education, and future career have the potential to increase their perceived control and competence. With positive feedback and a supporting environment, youth may experience an increase in self-efficacy. Sustained collaboration within treatment and work programs can also help youth learn to work with one another and with authority figures in achieving common goals (e.g., demonstrate respect for others, complete a project for a community partner). Programming that strengthens social and coping skills may also help youth learn how and when to advocate for themselves as well as address unanticipated problems that might arise in a variety of life domains (e.g., work, social life).

Strengths-based programming is on the rise within the juvenile justice field. Unfortunately, there is little research that has investigated these programs, particularly those that may foster psychological empowerment. At present, there is no empirical evidence to suggest that a focus on psychological empowerment results in improved short-term behavior (i.e., inside the correctional facilities) or long-term behavior (i.e., in the community). More problematic, perhaps, is that there is no research that seeks to conceptualize and operationalize the construct of psychological empowerment within youth correctional and re-entry facilities. Without an empirically supported measure of psychological empowerment, program evaluation and other research opportunities on this topic could not be reliably or validly conducted. 
The purpose of the current study was to take initial steps toward examining psychological empowerment in OYA correctional and re-entry facilities ${ }^{2}$. The present study introduced a new measure of psychological empowerment within the youth correctional context and investigated the factor structure of the scale used to measure this construct. This research also examined relationships between psychological empowerment and behavioral success. Specifically, behavioral indicators demonstrated by youth within five domains or skill areas established by OYA were investigated in relation to psychological empowerment.

To do this, a cross-sectional, non-experimental design was utilized. Self-report survey data on psychological empowerment was collected from incarcerated youth living within OYA correctional and re-entry facilities. Indicators of behavioral success, known as competencies in the five domains or skill areas, were then collected from OYA staff. Staff data was collected within one month of the collection of self-report survey data on youth perceptions of psychological empowerment.

The following sections will provide a framework for this study by introducing the juvenile justice system (Chapter II) as well as the construct of interest, psychological empowerment (Chapter III). Chapter II will summarize a brief history of the juvenile justice system, discuss developmental challenges associated with the juvenile justice

\footnotetext{
${ }^{2}$ OYA oversees two types of public facilities: (1) correctional and (2) re-entry. Correctional facilities are those facilities that house youth from the time they enter OYA custody until the time of release. Therefore, correctional facilities serve the general population of youth within OYA custody. Re-entry facilities are specific to the transition-to-community process. Although youth can transition into the community directly from correctional facilities as well as re-entry facilities, youth who live in re-entry facilities after some time in a correctional facility are offered additional opportunities to gain life skills as well as work in the community under the supervision of an OYA staff member or a community employer. However, the transition process often begins within correctional facilities, and many youth have similar opportunities to gain life and work skills in correctional facilities as they do in re-entry facilities. For these reasons, the proposed research will include youth within correctional and within re-entry facilities.
} 
system, and highlight the need for strengths-based treatment programming. In Chapter III, theoretical and research literature on youth empowerment will be outlined. Chapter IV advances this discussion by assessing the operationalization of empowerment within previous empirical work and describes the construction of the Psychological Empowerment scale used in the present study. Connecting Chapters II and III, Chapter V argues the importance of psychological empowerment to incarcerated youth.

Relationships between psychological empowerment and OYA and its youth are made. It is also in Chapter V that the study context is thoroughly introduced.

Following this introduction, research hypotheses will be developed in Chapter VI. Relevant research and theoretical literature discussed primarily in Chapters III and V will be reflected upon in order to justify the research questions and hypotheses. Chapter VII will discuss the research design in more depth, and the results of the current study will be detailed in Chapter VI. Finally, this dissertation will conclude with a recapitulation of the research findings as well as a discussion of the limitations, potential strengths and implications of the study, as well as future directions for research (Chapter VII). 


\section{CHAPTER II}

The Juvenile Justice System

In order to understand the potential importance of empowerment to incarcerated youth, one must first understand the state of the juvenile justice system. The following sections will introduce the juvenile justice system and provide a brief history of its evolution across the U.S. over the last century. This chapter will also discuss challenges associated with youth development within the juvenile justice system. Finally, the importance of strengths-based programming for incarcerated youth will be highlighted and justification for the investigation of empowerment in youth correctional facilities will be established.

Introduction and Brief History

The institution known as the juvenile justice system is defined as a network of organizations, including State and local court systems, State and local agencies, public correctional facilities, private treatment and social service centers, school systems, and State and local law enforcement, that work together to address crime committed by youth (Butts \& Mears, 2001). The juvenile justice system has not always functioned as it does today. Originally, it was more individualized and goal-oriented, emphasizing preventative and rehabilitative services (Butts \& Mears, 2001; Steinberg, Chung, \& Little, 2004). Presently it is much more streamlined, following what can be a strict set of rules and regulations (Butts \& Mears, 2001; Steinberg, Chung, \& Little, 2004).

In their discussion of the history of the juvenile justice system, Butts and Mears (2001) noted that it was first established in the late 1800s and early 1900s. At this time, communities began to realize that, in regards to criminal activity, youth should be treated 
differently than adults. Instead of waiting for a youth to commit a crime, authorities wanted to circumvent criminal activity by addressing problems before they surfaced (Butts \& Mears, 2001). This preventative approach was adopted because it was recognized that risk factors for illegal behavior were often the result of a failed system. The goal of a youth-specific justice system at this time was to provide individualized services to youth that help meet their developmental needs (Butts \& Mears, 2001; Steinberg et al., 2004). For these reasons, the city of Chicago established the first juvenile court system in the U.S. in 1899 (Butts \& Mears, 2001). Following Chicago's lead, cities across the U.S. began establishing similar juvenile court systems. Within 20 years, almost every city and state instituted a court system specific to youth (Butts \& Mears, 2001). Even as they spread across the country, criticisms of the juvenile court system began to surface by the middle of the $20^{\text {th }}$ century.

The growing system was criticized as too individualized, treating youth differentially depending on their circumstances (Butts \& Mears, 2001). Critics of early the juvenile justice system claimed that youth were denied the right to due process, as some youth could be arrested and treated without having committed a specific crime (Butts \& Mears, 2001). As a result of increasing dissent, the Supreme Court began formalizing the laws surrounding the institution, providing more rights to youth (e.g., formal notice of charges, right to an attorney, protection against self-incrimination) in the 1960s (Butts \& Mears, 2001). This paradigmatic shift, from a focus on individualized rehabilitation to a streamlined system, was more efficient and constitutional in many ways. At the same time, they also lead to the system becoming more like the adult justice system. 
Since the Supreme Court's intervention in the 1960s, several incremental laws have been introduced that are more punitive in nature, reflecting laws in adult court (Butts \& Mears, 2001; Kempf-Leonard, 2007; Steinberg et al., 2004). In response to a spike in violence committed by youth in the late 1980s and early 1990s (Blumstein, 2002; Sickmund, Snyder, \& Poe-Yamagata, 1997), authorities began passing laws that took a "get tough" on youth crime perspective (Butts \& Mears, 2001; Steinberg et al., 2004). For example, Butts and Mears (2001) and Steinberg and colleagues (2004) discuss the establishment of laws that decreased the confidentiality of juvenile court records. Laws were also created that based punishments on severity of crimes, and increased the number of youth transferred to adult courts (Butts \& Mears, 2001). These harsher laws contributed to the paradigm shift, which valued punishment over rehabilitation of youth offenders. As an example of one such law, the State of Oregon passed Ballot Measure 11 in 1994. Measure 11 introduced a set of mandatory sentences for specific crimes and was applied to both adults and juveniles. Under Measure 11, any youth who was arrested of a measure-related crime (e.g., murder, manslaughter, assault, rape, sexual abuse) would be transferred to the Department of Corrections and tried within an adult court. Through laws like those that levied harsher and longer sentences for youth, the juvenile justice system began to mirror the proceedings of the adult system.

Despite a decrease in violent crimes committed by youth since the mid-1990s (Sickmund et al., 1997), the juvenile justice system has largely remained punitive in nature (Steinberg et al., 2004). In 1994, it was estimated that 148,430 juveniles were arrested for violent crimes (Puzzanchera, Adams, \& Kang, 2012). In 2001, violent crimes committed by juveniles were estimated at 96,150 and in 2009 at 85,890 
(Puzzanchera et al., 2012). By these estimates, violent crime has reduced by approximately $42 \%$ since the "get tough on youth crime" era of the late 1980 s and 1990 s. Parallel to the decrease in violent crimes committed by youth, there has been a reduction in juvenile commitment to adult prisons (Snyder \& Sickmund, 2006). While this reflects a positive trend, it is evident that the "get tough" mentality has persisted over time. Instead of changing sentencing laws for juveniles that recognize differences in development between adults and juveniles, sentencing simply reflects trends in crimes committed by juveniles (Benekos \& Merlo, 2008). As violent crimes decrease, so do commitments to adult prisons. There still remains a percentage of youth offenders that are sentenced and treated as adults. In fact, Amnesty International (2005) reported that 2,255 youth across the country were serving life sentences without an opportunity for parole.

It has been argued that this approach has failed to significantly reduce recidivism and has "arrested" the development of incarcerated youth (Jenson \& Howard, 1998; Steinberg et al., 2004). As a result, reintegration for youth has, in some ways, become more difficult than it had been during the era that valued rehabilitation (Steinberg et al., 2004). Harsher sentencing and problem-focused treatment have often neglected to cultivate developmentally appropriate psychosocial skills (e.g., mastery and competence, interpersonal relationships, social functioning, self-definition; Steinberg et al., 2004). Now youth often re-enter the community without skills to succeed in a variety of life domains that they are bound to encounter (e.g., family, peer relationships, romantic relationships). For this reason, there has been a serious call for a second paradigmatic 
shift, renewing a focus on sentencing based on required rehabilitative services (Steinberg et al., 2004).

A renewed rehabilitative approach would allow practitioners to integrate programming for positive development into treatment. Harsher sentencing has led to an emphasis on addressing crime-related deficits. While some risk- and need-based treatments have led to a reduction in recidivism (e.g., RNR; Andrews et al., 20011), they have yet to incorporate a concomitant focus on developing youth strengths. A more positive, rehabilitative approach could provide youth with the opportunity to engage in developmentally appropriate activities. It is here that youth could address issues within a variety of life domains, including education, mental health, and social skills while incarcerated (Butts \& Mears, 2001; Steinberg et al., 2004). New and innovative programming for incarcerated youth has the potential to address the developmental challenges that youth are likely to face during and after incarceration in addition to a reduction in criminal behavior.

\section{Developmental Challenges During Incarceration}

Although development takes place throughout the lifespan, adolescence is a time when many changes and influences converge. Adolescents can experience changes in physical appearance, peer pressures, definitions of identity and autonomy, and relationships with friends and family (Bukowski, Sippola, \& Brender, 1993; Glick \& Sturgeon, 1998). As youth develop their own sense of values, they also experience increased persuasion from media (Bukowski et al., 1993; Glick \& Sturgeon, 1998). Increased cognitive and emotional skills are also associated with this development stage (Bukowski et al., 1993; Glick \& Sturgeon, 1998). During this time, youth are expected to 
develop certain psychosocial capabilities in order to take on more roles (e.g., parenthood, contributing member of society) and responsibilities (e.g., financial independence) as they enter adulthood (Steinberg et al., 2004). Navigating these changes and defining the self is an ongoing process and can be difficult, whether incarcerated or not (Bukowski et al., 1993). In addition to individual differences, the extent to which youth are provided "opportunity structures" for growth and development within specific contexts plays a role in sculpting their psychosocial capacities (Steinberg et al., 2004). For example, in an environment that does not allow youth to take on new roles and responsibilities, it is likely that many youth will have restricted development of mastery and competence, which are key psychosocial capacities according to leading developmental psychologists (Steinberg et al., 2004). For incarcerated youth, this process can seem exponentially more difficult than for youth living in the community.

The transition from adolescence to adulthood can be more difficult for incarcerated youth due to the nature of the juvenile justice system. Research has suggested that, as a result of an increased focused on reducing problem-behavior (KempfLeonard, 2007; Steinberg et al., 2004), incarcerated youth have become less equipped with the appropriate psychosocial capacities necessary for pro-social, healthy, independent living (Steinberg et al., 2004). In fact, incarceration can deprive youth of the opportunity for natural development of healthy romantic and platonic relationships, personal mastery, competence, identity, and self-determination (Abrams, 2006). When a youth re-enters society with these limited experiences, he is at a developmental disadvantage compared to his non-incarcerated, age comparable peers. 
In addition to the challenge of being "developmentally delayed" in comparison to community-based youth, incarcerated youth experience a second challenge when reintegrating into the community. Transitioning youth must simultaneously undergo the de-institutionalization and community re-entry processes (Altschuler \& Brash, 2004). De-institutionalization is defined here as the process by which youth learn to live outside the confines of a correctional facility. Youth must immediately adjust to living in an environment where supervision is not provided 24 hours per day. They have more autonomy and physical freedom to make decisions and act upon those decisions as they see fit. This experience drastically contrasts the restricted environment in the correctional facilities. While it may seem liberating to some, other youth can struggle with this transition (Altschuler \& Brash, 2004). A history of living within the confines of a pre-scripted, routine lifestyle previously dictated by authority figures can make relationship building with community peers or defining one's independence particularly hard for youth (Altschuler \& Brash, 2004; Glick \& Sturgeon, 1998). Through the transition process, incarcerated youth experience several challenges as they navigate changes related to adolescence and to de-institutionalization.

\section{Theory and Research on Strengths-Based Programming for Incarcerated Youth}

Opportunities for growth offered in correctional facilities are critical to the future success of incarcerated youth because they can address the delayed development that occurs as a result of incarceration in public correctional facilities. For instance, a review of the incarcerated youth intervention literature indicated that programs offering supports for interpersonal and pro-social development as well as future planning (e.g., relapse prevention plans) resulted in better outcomes (e.g., less recidivism) compared to those 
that did not provide these supports (Spencer \& Jones-Walker, 2004). Altschuler and Brash (2004) suggested that services that include educational and vocational training can help to elevate the skills, abilities, and knowledge of incarcerated youth similar to those seen in their community comparison peers. Additionally, incarcerated youth have reported that preparation for community re-entry that fosters the development of a "strong sense of resolve" helps youth succeed once they return to the community (Abrams, 2006). Much of the literature presented here focuses on programming specific to the transition-to-community process, but not all youth receive these types of services prior to the re-entry process. Even though research on strengths-based programming has not studied its effects at every point during incarceration, it is plausible that all youth within correctional facilities, even within the first few weeks of incarceration, can benefit from this approach. Aspects of this type of new, innovative, and progressive curriculum can benefit youth in correctional facilities by developing appropriate psychosocial capacities and helping them learn to re-engage in the community.

Despite these theoretical assumptions and initial research findings, the scope and depth of protective factors and promotion of strengths has not been thoroughly investigated. There has been a call for more research on developmentally appropriate programming, particularly on interventions that cultivate psychosocial capacities and reintegration skills (Altschuler \& Brash, 2004). Therefore, research identifying and examining the outcomes of strengths or potential protective factors, such as psychological empowerment, in incarcerated youth is well warranted. The following section discusses psychological empowerment as a potentially beneficial strength for incarcerated youth. 


\section{CHAPTER III}

\section{Empowerment}

The following chapter will introduce the construct of empowerment. First, the chapter will broadly define empowerment and then outline its importance to youth.

Following a discussion of the theoretical underpinnings of youth empowerment, research on the antecedents, correlates, and outcomes of psychological empowerment will then be presented. Concluding this chapter will be a brief introduction to the connections between the population of interest, incarcerated youth, and empowerment.

\section{Introduction}

Empowerment was formally introduced as a construct in Community Psychology by Rappaport in the 1980s (Rappaport, 1981). While Rappaport was the first to introduce it to the field, the roots of empowerment lie in the social movements of the 1960 s and 1970s. Freire (1970) developed the foundations of empowerment through his work within the Brazilian education system. In the Pedagogy of the Oppressed, Freire (1970) discussed the need for marginalized, oppressed peoples of Brazil to undergo a process known as "critical consciousness." Through this process, oppressed groups learn about and reflect upon social, political, and economic contradictions within society as well as learn to take action against oppressive factors that have control over aspects of their lives (Freire, 1970). The notion that oppressed groups need to reflect upon and take action against oppressive forces was the foundation for Rappaport's theory of empowerment.

Throughout the past few decades, empowerment has taken on a number of definitions within a variety of different fields (Perkins \& Zimmerman, 1995). It is important for Community Psychology to put forth a consistent and concrete definition of 
empowerment in order to guide consistent and focused research. Therefore, Rappaport's conceptualization of empowerment will serve as the overarching theoretical basis for this dissertation, while Zimmerman's research on psychological empowerment will serve as the specific, operational definition of empowerment (see Chapter IV).

According to Rappaport (1981), empowerment can broadly be defined as individual determination over one's own life. More specifically, empowerment is the process or ability to move beyond oppressive factors that inhibit an individual or group from doing something (Rappaport, 1981; Rappaport, 1987). Perkins and Zimmerman (1995) offer three key definitional attributes to empowerment that align with Rappaport's conceptualization. They suggest that empowered individuals or groups: (1) can gain control of their lives; (2) are able to participate in decisions that impact them; and (3) have reflected upon and understand the social, political, and economic forces that influence their lives (Perkins \& Zimmerman, 1995). Within this definition, empowerment can refer to both individual and group level processes.

There are two critical distinctions that further guide the conceptualization of empowerment. First, Rappaport (1987) distinguishes between empowerment as a process and as a state. As a process, empowerment is a series of thoughts or ideas that reflect upon oppressive factors (Perkins \& Zimmerman, 1995). The process can also include a series of actions taken against oppressive factors that gradually release conditions of oppression, allowing individuals or groups to gain more control over their lives. The result of the process of empowerment is a state of empowerment, where individuals or groups can act free of oppressive factors that previously limited their social, political, and economic conditions. Empowerment as an outcome often surfaces as perceived control 
or resource mobilization (Perkins \& Zimmerman, 1995). The focus of this dissertation is on empowerment as an outcome.

Second, Rappaport (1987) and other Community Psychologists (Gruber \& Trickett, 1987; Riger, 1993) discuss two distinct types of empowerment, as a psychological sense and as lived or actual experience. First, empowerment is discussed as a psychological sense. Empowerment as a psychological sense is associated with an individual's perception of his or her ability to do the things s/he wants to do and that her or his voice is heard on issues of importance (Gruber \& Trickett, 1987; Rappaport, 1987; Riger, 1993). Empowerment as a lived experience refers to an individual's or a group's actual ability to affect change (Rappaport, 1981). In this way, empowerment also refers to political or decision-making power over important or needed resources (Gruber \& Trickett, 1987; Rappaport, 1987; Riger, 1993). Although actual decision-making power is a key aspect of empowerment, the focus within this dissertation is on psychological sense of empowerment because the nature of the context (i.e., correctional facilities for incarcerated youth) is more rigid and authoritarian than non-incarceration related contexts (Altschuler \& Brash, 2004; Schwartz, 2000).

In addition to these distinctions, it is recognized that empowerment is multidimensional and pluralistic. The degree to which an individual or a group experiences empowerment depends on context. Some environments are more natural settings for empowerment to occur (e.g., community rally meetings) while other settings might limit empowerment (e.g., boarding school). Empowerment can also be contradictory. For instance, Riger (1993) provides an example of the differential experience of empowerment between two victims of sexual violence. In her example, an 
Anglo-American woman finds that cooperating with law enforcement to charge the perpetrator is an empowering experience. In contrast, an African-American woman, who is committed to upholding values of solidarity within her community, may not want to cooperate with law enforcement at all, valuing community norms above personal need for justice. Beyond Riger's example, the African-American woman could experience psychological empowerment in a different context. For instance, she might feel that she gains control over important issues by participating in community-based decisions during neighborhood meetings. This example demonstrates that individuals can experience empowerment in very different ways.

The literature on empowerment began by assessing the construct in adult populations. Although not addressed in early literature, youth and young adults can also experience empowerment, albeit sometimes in different ways. Youth and young adults are oftentimes still under the authority of their parents or other caregivers as well as their teachers, coaches, or program-related staff (e.g., extra curricular activities). Outside of the context of the family, youth can experience institutional-, community-, and societallevel oppressive factors similar to those experiences by adults (e.g., racism, discrimination based on disability status). Oppressive factors, such as a patriarchal culture, can impact youth within contexts specific to their lives. Contexts where youth and young adults might experience systemic issues uniquely include school, extracurricular activities, and even leisure time in public spaces. The impact of these factors, whether similar or different than those experienced by adults, is no less important to address. Where power dynamics place youth in a subordinate position, empowerment programming may help youth overcome challenges related to an unfavorable system 
(Cargo et al., 2003; Chinman \& Linney, 1998; Jennings et al., 2006; Messias, Fore, McLoughlin, \& Para-Medina, 2005). Empowerment can also have a distinct importance to youth, particularly as they transition into early adulthood.

\section{Importance of Empowerment During Adolescence}

Empowerment can be used as an effective tool during developmental transitions associated with adolescence. Several developmental changes take place during the span of time between childhood and adulthood. During this period, youth explore their selfconceptualization and identity (Chinman \& Linney, 1998; Erikson, 1968; Steinberg \& Morris, 2001) as well as experiment with different roles and responsibilities in their community and in society (Chinman \& Linney, 1998; Mohajer \& Earnest, 2009). They also begin to focus on and further develop relationships, particularly with peers and the larger society (Chinman \& Linney, 1998; Mohajer \& Earnest, 2009; Steinberg \& Morris, 2001). In doing so, they acquire important social competencies that are required for prosocial, healthy relationships in adulthood (Chinman \& Linney, 1998) and bond to prosocial institutions (Steinberg \& Morris, 2001). Essential developmental milestones during adolescence, which include exploring the sense of self and identity, taking on roles and responsibilities, gaining social competencies, and internalizing social institutions, can be aided by empowerment-based programming targeted at youth.

The experience of empowerment can help youth navigate one of the most commonly discussed developments during adolescence, the exploration and solidification of the self or one's identity (Chinman \& Linney, 1998; Erikson, 1968; Peterson, 1988; Steinberg \& Morris, 2001). Throughout adolescence, youth explore and define their own sense of who they are based on personal beliefs and values. In this process, they seek to 
increase their self-esteem, self-worth, and self-efficacy (Chinman \& Linney, 1998; Steingberg \& Morris, 2001). These concepts are foundational to empowerment. By participating in programs that allow youth to create their own definition of the self, freely explore their life trajectory, and put into action their own plans for change or plans for their future (i.e., empowerment-based programming), youth gain a better understanding of their own identity (Chinman \& Linney, 1998). Youth empowerment programming, which aims to allow youth space for personal growth and support, can facilitate developmental growth areas related to self-identity, self-efficacy, and self beliefs (Chinman \& Linney, 1998).

Empowerment can also help youth gain positive reinforcement from adults and peers that encourage positive or pro-social decisions (Chinman \& Linney, 1998; Cargo et al., 2003). The expectations-state theory supports the idea that the youth empowerment process can reinforce societal norms and values. In their model of the youth empowerment process, Kim and colleagues (1998) purport that youth act according to the expectations placed on them from their family and the surrounding social system. By placing positive expectations that are aligned with empowerment (e.g., self-efficacy, skill development, working together to create positive change) on youth, positive or pro-social behaviors can be cultivated.

New roles and responsibilities can also be explored during adolescence within empowerment programming. Through the empowerment process, youth have the opportunity to discover their interest in different roles and responsibilities, particularly various leadership roles that do not generally arise within the community (Chinman \& Linney, 1998). Encouraging youth to take these risks, empowerment programming gives 
youth more control over decision-making and individual or group action (Chinman \& Linney, 1998). Continually experiencing the opportunity to take on roles and responsibilities and having control over important decisions allows youth to gain a sense of role stability (Chinman \& Linney, 1998). Reinforcement of the importance of their involvement and the validation of their decisions from adults and peers further increases youth self-esteem and solidifies their sense of self (Chinman \& Linney, 1998).

Empowering youth to take on new and challenging roles and responsibilities helps them to develop their identity and provides a foundation for the roles and responsibilities associated with adulthood (e.g., full-time job, family, contributing to society). In these ways, empowerment can support youth through critical developments that help the transition from adolescence to early adulthood.

Adolescence is also a time when youth gain skills and abilities related to social competence (Steinberg \& Morris, 2001). Empowerment-based programming encourages youth to work together to reflect on social institutions that impact their lives and to collectively create change that is important to them. In working with other youth and with adults who might be perceived as authority figures, youth have the opportunity to gain skills related to the initiation and maintenance of interactions, cooperation, activelistening, and collaboration. In learning to work effectively with others, youth gain a sense of social integration (Cargo et al., 2003), which helps them bond to pro-social values. Empowerment-based programming, which stresses collaboration and thoughtful reflection on interpersonal interactions, can help youth to cultivate pro-social skills behaviors. 
Within empowerment programming, bonding to institutions (e.g., pro-social roles) can occur through meaningful contributions to a group or to society or through social learning (Chinman \& Linney, 1998; Hawkins, Catalano, \& Miller, 1992; Kim, Crutchfield, Williams, \& Helper, 1998). The empowerment process encourages youth to actively participate in creating change that is important to them. Active involvement in activities related to desired change increases youths' commitment to positive, pro-social change, resulting in a bond to social institutions (Chinman \& Linney, 1998). Institutional bonding can also occur through social learning whereby youth learn to imitate or model the behavior of others, particularly when positive reinforcement or avoidance of punishment is present (Kim et al., 1998). Underlying youth empowerment is the notion that adults serve as positive role models (Cargo et al., 2003; Jennings et al., 2006; Messias, Fore, McLoughlin, \& Para-Medina, 2005). As youth learn to distinguish between what is and what is not reinforced, they adopt or internalize the norms and values of their role models (Kim et al., 1998). In empowerment programming, social values include self-efficacy and self-worth, control, competency and mastery of relevant or desired skills and abilities, pro-social participation, and change-oriented action. As youth begin to imitate program-facilitating adults and internalize program values, they learn to take on leadership roles and encourage others to adopt these same values. Bonding to social institutions, which is a key development process that occurs during adolescence, can take place within the empowerment process. With active participation in empowerment programming, youth learn to internalize and espouse social norms cultivated by staff and peers. 
There is one important caveat to institutional bonding that youth experience in empowerment-based programming. Although youth learn to internalize pro-social values that are institutionalized in their group, community, or larger society, empowerment processes should encourage youth to critique oppressive factors that inhibit their freedom of choice. Foundational to empowerment is critical reflection on social factors that influence one's environment as well as active participation to work against these factors, allowing for more choice over important decisions. Furthermore, youth will likely learn think critically about their world, in addition to the adoption of pro-social values, as they model adults facilitating the empowerment process and learn about the expectations of the group.

Empowerment programming compliments positive youth development, helping youth explore their sense of self and identity, take on different roles and responsibilities, gain social competencies, and internalize social institutions. These processes may be cyclical, wherein youth gain opportunities to demonstrate their skills and abilities. In turn, their identity, self-esteem, and self-efficacy are strengthened. Increasing youths' understanding and belief in themselves encourages them to increase their participation and involvement in positive activities. As a result, they can gain more skills and abilities related to their own as well as collective interests. Empowerment also helps youth define and strengthen the self as well as connect with others in a larger movement toward positive, pro-social change to the extent that positive beliefs about the self and pro-social behaviors are reinforced (i.e., expectation-states theory) and they have effective models to imitate (i.e., social learning theory). As a result of the positive effects that empowerment programming can have on youths' abilities to navigate development stages 
associated with adolescence, it is important to understand youth empowerment theories more broadly.

\section{Youth Empowerment Theories}

Empowerment theory has been applied to a variety of contexts, targeting several different populations. For example, Fawcett and colleagues (1994) applied empowerment theory in several ways to help people with disabilities gain access to needed resources to improve their quality of life in a number of contexts. Rappaport (1998) described empowerment theory as it related to an economically disadvantaged, marginalized African-American community working hand-in-hand with the broader community. Empowerment theory has also been used to help youth navigate their own lives and become engaged in the political system in order to help make decisions that impact them.

As early as the late 1980s, theorists and practitioners began applying empowerment theory to youth. For instance, Wallerstein and Berstein (1988) described a substance abuse prevention program that used an empowerment approach to help multiethnic youth make healthy lifestyle choices and participate in their community civically and politically. Even though there are examples of the application of empowerment in youth populations within the early literature, youth empowerment theory did not fully take root until the 2000s. Bemak and colleagues (2005) used an empowerment approach to help "at-risk" students engage in school and to help them succeed academically. Fusoni (2005) described the application of empowerment theory in a program aimed at encouraging African-American youth to change youth relations with law enforcement. Reflecting the empowerment literature on people with disabilities, Yuen and 
Shaughnessy (2001) called for an extension of empowerment theory focusing on programs for students with disabilities. In 2004, the journal Health Education and Behavior published a special issue on youth empowerment and tobacco-related interventions. Reflected by the variety of young adult populations in which empowerment theory has been applied, this construct can be conceptualized in diverse contexts.

There a number of exemplary models of youth empowerment, and theorists have synthesized these models into several youth empowerment theories. Jennings and colleagues (2006) compiled four models of youth empowerment to develop and introduce the Critical Youth Empowerment (CYE) theory. They suggested that, when youth are provided the opportunities associated with CYE, youth are more likely reap the benefits of empowerment at the individual- (e.g., increase self-esteem; sense of purpose; positive, pro-social identity) and community-levels (e.g., community engagement; Jennings et al., 2006). CYE elements impacting youth empowerment included a welcoming and safe environment, an opportunity to participate and engage in a meaningful way, a chance to share power with adults equitably, the experience of critical consciousness, an opportunity to participate in socio-political processes to affect change, and a chance to integrate individual- and community-level empowerment (Jennings et al., 2006). Although there has been some theoretical discussion of youth empowerment, research on youth empowerment is still developing. Even as these models continue to evolve, initial evidence supports these models.

Research on Youth Empowerment 
A number of studies have been conducted to support the theoretical assumptions of youth empowerment theories. All of the studies on youth empowerment evaluated programs aimed at changing youth health behavior. The majority of studies evaluated prevention or intervention programs aimed at reducing risky health behavior, such as tobacco use, drug use, and sexual risk taking (Berg, Coman, \& Schensul, 2009; Evans, Ulasevich, \& Blahut, 2004; Hinnant, Nimsch, \& Stone-Wiggins, 2004; Holden, Crankshaw, Nimsch, Hinnant, \& Hund, 2004a; LeRoy, Benet, Mason, Austin, \& Mills, 2004; Messias et al., 2005; Ribisl, Steckler, Linnan, Patterson, Pevzner, Markato, Goldstein, McGloin, \& Peterson, 2004). Many of these programs, however, incorporated aspects of health promotion into their curriculum. Only one study was found to investigate youth empowerment theory within a program that exclusively sought to enhance health and well-being of youth (Cargo et al., 2003). Results from these studies can be discussed in terms of antecedents, correlates, and outcomes of youth empowerment.

Several studies have examined the antecedents of youth empowerment. Factors most often reported as impacting the development of youth empowerment relate to the environment created by facilitating adults. Facilitating adults are those individuals responsible for creating a welcoming, safe, and social environment (Cargo et al., 2003; Jennings et al., 2005). These positive environmental characteristics are associated with an increased likelihood that youth will experience empowerment (Cargo et al., 2003; Messias et al., 2005). Adults foster a welcoming environment by caring for and respecting each youth as well as believing that youth are capable of positive, pro-social change (Cargo et al., 2003). Within a safe environment, youth are more likely to actively 
participate and learn from programming (Lee, Borden, Serido, \& Perkins, 2009). This safe environment can encourage youth to explore different roles and responsibilities, demonstrate skills that they learn, and participate in decision-making and other important activities while integrating feedback from adult facilitators into their thoughts and behaviors (Jennings et al., 2005). Within welcoming, safe, structured settings, youth begin to take multiple perspectives and enable further growth through facilitation, teaching, mentoring, and providing feedback to one another (Berg et al., 2009; Cargo et al., 2003). Structure and high standards for behavior and performance also help to create an empowering setting. By raising the bar for youth performance, creating space to get things done, maintaining positive relationships with youth, exerting control or influence when necessary and appropriate, and communicating and connecting with the broader community, programming is more likely to foster youth empowerment (Messias et al., 2005). As demonstrated through research, adults' ability to shape an environment that provides appropriate structure and demands, that is perceived as welcoming and safe, and that allows for intra- and inter-personal growth through a variety of activities is essential to the empowerment process.

Although these studies found the role of the adult in the prevention or intervention program to be particularly critical in cultivating youth empowerment, research by Evans and his colleagues (2004) suggested that the relationship between adult involvement and youth empowerment might be indirect. They found that group structure and group climate mediated the relationship between adult involvement and collective participation, an indicator of empowerment (Evans et al., 2004). Whether direct or indirect, it is clear 
that the role of adults in the prevention or intervention program can help to foster youth empowerment.

In support of the work by Evans and his colleagues (2004), research has shown that systemic reinforcement, particularly at the community and societal levels, can also precede youth empowerment. In other words, community and societal factors may increase empowerment at the individual and group levels. Some research has found mixed results at the community level, where general community support was not related to the number of group empowerment activities offered (Hinnant, Nimsch, \& StoneWiggins, 2004). While general community support mattered less, Hinnant and colleagues (2004) found that support from other youth outside of the empowerment intervention program predicted the number of policy related activities offered (Hinnant et al., 2004). It has also been suggested that community and societal values related to the purpose of the intervention program heavily impacted youth empowerment. For example, LeRoy and colleagues (2004) found that teen cultural norms around tobacco use within the community as well as funding and political support for tobacco control programming predicted youth empowerment in a tobacco control intervention. Their research also indicated that the history of tobacco control within the community played a major role in the success of the empowerment intervention program (LeRoy et al., 2004). In addition to adult facilitation and the structured of the environment, historical context as well as community and societal values precede youth empowerment.

There is a lack of research on the correlates of youth empowerment. LeRoy and her colleagues (2004) were the only researchers found to have studied potential correlates of youth empowerment. Their research indicated that organizational empowerment is 
associated with youth empowerment. In organizations where program staff and administration felt empowered and had the opportunity to contribute and participate in meaningful ways, youth may also experience a sense of empowerment within programs offered by the organization. While further research is clearly warranted, this study demonstrated that a "trickle down" effect could take place, where empowered staff are more likely to create empowered settings for youth.

Similar to the dearth of research literature on correlates, few studies have examined the outcomes of youth empowerment at the individual and group levels of analysis. At the individual level, it has been found that youth psychological empowerment predicted increased engagement and participation, actualizing potential (i.e., esteem, confidence, competence, critical consciousness), control (e.g., taking responsibility, voicing opinions, taking action, decision making, confronting challenges, learning, and improving one's quality of life), and constructive change (e.g., youth development, success, and social integration; Cargo et al., 2003). Evaluation research has also found that youth participating in an empowerment-based prevention program significantly decreased negative health behaviors (e.g., marijuana usage; Berg et al., 2009). These findings indicate that youth empowerment can result in both cognitive (e.g., increased self-esteem, increased confidence) and behavioral (e.g., improving quality of life, social integration) changes at the individual level.

As suggested by empowerment theory, behaviors enacted to gain more power or control to make desired change often result from the empowerment process (Rappaport, 1981; 1987). Research on political participation, an example of a behavioral outcome of empowerment, has yet to confirm this theory. At the individual level, some research has 
shown that the more youth are able to take on a variety of roles through youth empowerment programs, the more they report having perceived socio-political control (Holden et al., 2004a). On the other hand, findings at the group level are contradictory. One study indicated that a youth empowerment program aimed at changing tobacco control laws did not result in political group efficacy (Ribisl et al., 2004). These results were found despite significant political involvement in state government by participating youth (Ribisl et al., 2004). Within this study, researchers found that the tobacco control intervention program, aimed at empowering youth to influence laws and regulation on tobacco, did not have the intended effect at the group-level (Ribisl et al., 2004). These youth did not feel that their input helped to change tobacco policies (Ribisl et al., 2004). The authors qualified their results by acknowledging one serious limitation; government funded the youth empowerment program, and students did not always feel comfortable attempting to change the policies of the agency that funded their program (Ribisl et al., 2004). In comparing the two studies on political participation and effectiveness discussed above, analyses were conducted at different levels. It may be entirely possible that individual youth perceive political impact, as in the former study. At the same time, they may also feel incapable of making change as a group, as in the latter study. Due to inconsistencies, it is unclear whether empowerment can provide youth with the skills, abilities, and motivation to take action at the individual and group levels.

\section{Conclusion}

Research within the field of youth empowerment is still developing. Foundational to future research on youth empowerment are several concrete findings that support the continued investigation of this construct within young adult populations. It is 
clear that facilitating adults play a crucial role in creating an empowered setting, which integrates warmth and support with structure, guidance, and opportunity (Cargo et al., 2003; Jennings et al., 2005; Messias et al., 2005). Other contextual factors, including the history and values of the community, also impact the development and experience of empowerment (Hinnant et al., 2004; LeRoy et al., 2004). Perhaps more supportive of continued research on youth empowerment is the empirical evidence that suggests that the experience of empowerment has positive outcomes for youth, including cognitive and behavioral changes (Berg et al., 2009; Cargo et al., 2003). While these studies serve as an important base for additional research, it is apparent that the range of young adult populations empirically studied should be expanded. As previously mentioned, previous work has a narrowed focus on particular youth populations (e.g., minority youth) and emphasize programs with a specific intervention target (e.g., tobacco use). Broader populations, especially those with a potentially wider range of prevention, intervention, and treatment goals, should be studied.

One young adult population that has yet to be included in empowerment research is incarcerated youth. This context is a unique setting in which to study empowerment because of its historical developments. Correctional facilities for youth have, in recent history, been characterized as rigid and authoritarian (Altschuler \& Brash, 2004; Schwartz, 2000), and treatment is often aimed at problem-behavior and deficits-based (Schwartz, 2000; Zeldin, 2004). This line of research answers the call of practitioners in the field to study treatment programming that is strengths-based. Research on empowerment within youth correctional facilities also provides the opportunity to reflect upon the conceptualization and operationalization of empowerment in a setting unlike 
those that have been previously studied. As a result of the burgeoning nature of the field of research on youth empowerment, there is currently no theoretical or empirical literature that discusses empowerment within correctional settings and with incarcerated youth populations. A thorough discussion of research on the conceptualization and operationalization of empowerment is warranted, and the first steps toward measuring this complex construct need to be established. 


\section{CHAPTER IV}

\section{Psychological Empowerment Measure Development}

In defining empowerment, it is clear that there are several points at which its conceptualization varies. These "forks in the road" largely depend on the type of empowerment being discussed within specific contexts (see Chapter III). It is clear that empowerment must be conceptualized within specific contexts in order to measure the construct appropriately and in a culturally sensitive manner. The distinctions and assumptions mentioned above (e.g., state versus process, psychological sense versus lived experience, multilevel, contextually dependent, and pluralistic) dictate the way in which empowerment is studied. Specifically, the way in which researchers conceptualize the construct within a given context guides its measurement.

This chapter will first discuss the research literature aimed at measuring empowerment. Psychological empowerment (PE), the most commonly measured construct within the empowerment literature, will be defined and examples of PE scales will be provided. As PE is most commonly operationalized and has the most empirically supported measures within the research literature, this study will utilize the conceptualization of PE. PE has never been conceptualized nor operationalized within a youth correctional context, the focus of the study. For this reason, this chapter will also discuss the development of a measure of PE for incarcerated youth within Oregon Youth Authority.

Assessing Empowerment in Research

Due to a variety of different conceptualizations of empowerment, the construct has been assessed in a number of different ways, both as a state (e.g., psychological 
empowerment) and as a process (e.g., actual decision-making power). The diversity of techniques used to measure empowerment is augmented by the notion that empowerment must be contextually defined. Therefore, a single, universal measure of empowerment would not be appropriate or valid (Zimmerman, 1995).

Several scales have been developed to measure empowerment within a number of different contexts. For instance, Akey, Marquis, and Ross (2000) developed the Psychological Empowerment Scale to assess empowerment for parents of children with a disability whereas Holden and colleagues (2004b) created a measure of empowerment for youth in a tobacco control program. Israel and colleagues (1994) developed a multilevel measure of community empowerment. Still another empowerment scale was constructed for use in an organizational context (Kraimer, Seibert, \& Linden, 1999). As demonstrated by the variety of empowerment scales, the way in which this construct is measured highly depends on the type of empowerment being assessed (e.g., psychological empowerment, actual decision-making power), the level of analysis under examination (e.g., individual, organization, community), as well as the group or population under investigation.

It has been suggested that psychological empowerment is most easily measured because it is related to concrete, individual-level outcomes (e.g., perceived control, competence; Jennings, Parra-Medina, Messias, \& McLoughlin, 2006). Early work on specifying the nomological network of psychological empowerment suggests that it, too, is difficult to measure because: (1) it is experienced differently by different people through a variety of cognitions and behaviors; (2) its development is context dependent; and (3) it is dynamic, changing over time (Zimmerman, 1995). 
There are several fundamental aspects of psychological empowerment. From early conceptual research, it has been suggested that psychological empowerment is a combination of personality, cognitive, and motivational factors and is associated with an internal locus of control, desire for control, competence, mastery, and political efficacy (Zimmerman \& Rappaport, 1988). Although the measurement of empowerment, including psychological empowerment, depends on a number of factors, the work of Zimmerman and his colleagues $(1992 ; 1995)$ has identified underlying components of psychological empowerment. Based on previous empirical work (Zimmerman, Israel, Schulz, \& Checkoway, 1992), Zimmerman (1995) proposed three principal components underlying psychological empowerment: (1) the intrapersonal component; (2) the interactional component; and (3) the behavioral component. The intrapersonal component is composed of perceived control, perceived self-efficacy, motivational control, perceived competence, and mastery within specific life domains (e.g., family, work, school; Zimmerman, 1995). The interactional component relates to individuals' understanding of their environment and their ability to prepare for action through critical awareness, understanding causal agents, skill development, skill transfer across life domains, and resource mobilization (Zimmerman, 1995). Finally, the behavioral component requires individual action through community involvement, organizational participation, and appropriate coping behaviors (Zimmerman, 1995). These three components of psychological empowerment (i.e., intrapersonal, interactional, and behavioral) have guided the development of specific measurement scales of each of the components of the construct. 
The intrapersonal component of psychological empowerment is most consistently measured in the literature. Zimmerman and Zahniser (1991) developed the Sociopolitical Control Scale (SPCS) to assess intrapersonal psychological empowerment. The SPCS is composed of 17 Likert-scale items that assess two primary dimensions: (1) leadership and (2) policy control. These dimensions encompass political efficacy, perceived competence, locus of control, and sense of mastery (Peterson, Lowe, Hughey, Reid, Zimmerman, \& Speer, 2006). Since its development, the SPCS has been validated in its entirety as well as in abbreviated versions in a number of populations and contexts. Validation research has included parents of children with disabilities (Akey et al., 2000), youth participants in a tobacco control program (Holden et al., 2004a), randomly selected residents in the Northeastern part of the U.S. (Speer \& Peterson, 2000), randomly selected participants from an evaluation of a community health promotion initiative in the Midwest (Peterson et al., 2006), and randomly selected participants of needs assessment in Northeastern U.S. (Peterson et al., 2006). These studies and others have consistently demonstrated that the SPCS is a useful scale in the measurement of the intrapersonal component of psychological empowerment.

The assessments of the interactional and behavioral components of psychological empowerment are less consistent. The measurement of the interactional component of psychological empowerment reflects the diversity of contexts of study, where the context largely interacts with an individual's ability to comprehend the factors that impact his or her environment, transfer skills from other life domains, and mobilize resources (Zimmerman, 1995; Zimmerman \& Warschauski, 1998). For example, Akey and her colleagues (2000) use questions related to perceived knowledge and skills to measure 
interactional psychological empowerment, whereas others employ questions related to knowledge of resources, assertiveness, and advocacy to assess the interactional component of psychological empowerment (Holden et al., 2004a).

Similarly, the behavioral component of psychological empowerment is inconsistently measured. Some researchers use intent to participate as a measure of the behavioral component (Akey et al., 2000). Others use measures of self-reported previous actions to assess behavioral aspects of psychological empowerment (Speer \& Peterson, 2000). While behavior is required under Zimmerman's conceptualization of psychological empowerment, many researchers intentionally omit this component when assessing psychological empowerment for a variety of reasons (Holden et al., 2004; Kraimer et al., 1999; Menon, 1999). For instance, Holden and colleagues (2004) believed that action was the outcome of the empowerment process and thus measured it as their dependent variable. Kraimer and colleagues (1999) and Menon (1999), on the other hand, confounded actual behavior with perceptions of or attitudes about behavior related to the context. Partially as a result of the contextual nature of this psychological empowerment's conceptualization, the measurements of the interactional and behavioral components are typically developed for a specific context and population of study.

Empirical work has demonstrated that measures of psychological empowerment should be developed within and for specific contexts. At the same time, several measures can be used as a foundation to create a contextually-based measure of psychological empowerment. The SPCS (Zimmerman \& Zahniser, 1991) is commonly used to develop questions related to the intrapersonal component of psychological empowerment. Additionally, scales by Akey and colleagues (2000), by Holden and colleagues (2004a; 
2005), by Kraimer and colleagues (1999), and by Menon (1999) can provide examples of general and setting-based questions that have been used to reliably measure psychological empowerment.

\section{The Development of a Measure of Psychological Empowerment for Incarcerated Youth}

As previously mentioned, psychological empowerment is composed of three components: (1) intrapersonal; (2) interactional; and (3) behavioral. Currently, there is no measure of psychological empowerment that assesses all three of these components for a youth or young adult, incarcerated population. Therefore, three self-report survey sub-scales measuring the components of psychological empowerment in three OYA settings (i.e., treatment groups, school or vocational activities, and their residence or living unit) were created specifically for use in OYA correctional and re-entry facilities. These settings were chosen because the large majority of, if not all, youth participate in these settings on a regular basis. Additionally, they were chosen because activities or programming in each setting are completely separate; however, they may be conducted in the same physical space (see Chapter V for further discussion related to settings at OYA).

The general process used to create the sub-scales measuring psychological empowerment followed a specific protocol. Measure construction began with a review of the literature describing the validation of seven scales of psychological empowerment or one or more of its components. These measures were designed for specific contexts, such as tobacco-control interventions (Holden et al., 2004a; 2005) and programs for parents of children with disabilities (Akey, 2000) as well as for general use (Israel, Checkoway, Schulz, \& Zimmerman, 1994; Kraimer et al., 1999; Menon, 1999; Peterson, Lowe, Hughey, Reid, Zimmerman, \& Speer, 2006; Speer \& Peterson, 2000; Zimmerman, \& 
Zahniser, 1991). After reviewing several measures, the principal researcher selected three sub-components or domains of each component of empowerment to assess. Selection of domains was based on discussions regarding the importance of each subcomponent in the literature as well as the availability of validated measures of each. Following the selection of domains, scales of each domain were reviewed in order to select two items, one positively worded and one negatively worded, to evaluate that particular domain. Items were selected based on explicit criteria. First, the scale from which the item was selected was prioritized if it had high reliability and validity and/or was commonly cited within the literature (i.e., more than one study from different researchers utilized the measure). Second, items with the highest standardized factor loadings were prioritized. Third, items that were context specific were avoided. Next, items characterized as problematic (e.g., double-barreled) were eliminated. Items were included if they satisfied the above conditions and were negatively worded or if they were easily converted to a negatively worded item. Finally, items that fit the response format were prioritized.

Once two total items, one positively worded and one negatively worded item, were selected to measure each of the three domains for each component of psychological empowerment, the complete measure, which included 18 items per OYA context or setting, was sent to the Dissertation Committee for revisions and approval. Based on feedback from the committee, revisions were made to the items. Additional changes were made in collaboration with Dr. Todd Bodner, the expert statistician on the Dissertation Committee. The following sub-sections detail domain and item selection within each component of psychological empowerment. 
Intrapersonal Psychological Empowerment. Following the conceptualization of empowerment by Zimmerman (1995) and Zimmerman and Warschausky (1998), the Intrapersonal component of psychological empowerment typically includes topics related to perceived control, self-efficacy, motivation to control, and perceived competence. Self-efficacy, motivation to control, and perceived control were selected as the three domains assessing the Intrapersonal component of psychological empowerment. These domains were selected because they were heavily emphasized in the empowerment literature (Zimmerman, 1995; Zimmerman \& Warschausky, 1998), because they were common constructs assessed as part of the intrapersonal component of empowerment, and because several scales measuring these concepts have been validated and thus served as empirically supported measures of these three sub-components.

Four empirically supported scales measuring self-efficacy were examined (Chen, Gully, \& Eden, 2004; Muris, 2001; Scherer, Maddux, Mercandante, Prentice-Dunn, Jacobs, \& Rogers, 1982, Schwarzer, Babler, Kwiatek, \&Schoder, 1997). The two most commonly used measures of self-efficacy with the most empirical support for construct validity (i.e., Self-Efficacy Scale published by Scherer and colleagues [1982] and New General Self-Efficacy Scale published by Chen and colleagues [2004]) were chosen as scales from which self-efficacy items would be selected. Several scales measuring selfefficacy were developed based upon Scherer and colleagues (1982) original publication of the Self-Efficacy Scale (SES), which includes the General and Social Self-Efficacy sub-scales. The New General Self-Efficacy Scale (NGSES; Chen et al., 2004) was one such measure, which attempted to improve upon limitation within the SES and its subsequent revisions. 
From the SES, one negatively worded item was selected. This item, which reads, "If something looks too complicated, I will not even bother to try it," was chosen because it appeared as one of highest loading items in Sherer and colleagues' (1982) and Bosscher and Smit's (1998) work. It was also chosen because its wording was reversed and could easily fit the correctional context. No adaptations were made to this item.

The positively worded item was selected from the New General Self-Efficacy Scale (Chen et al., 2004). Although Chen and colleagues (2004) did not report factor loadings, this item, which reads "I am confident that I can perform effectively on many different tasks," was based upon an item with a high factor loading within the work of Schwarzer and colleagues ("I am confident that I could deal efficiently with unexpected events;" 1997). Chen and colleagues item was chosen because it was perceived to "flowed" better than Schwarzer and colleagues' item and was easily adapted to fit the correctional context. In order to simplify the language, the item was revised to read "I am confident that I can work effectively on many different tasks."

Two scales that explicitly measured motivation to control, the Socio-Political Control Scale (SPCS; Zimmerman \& Zahniser, 1991) and the Desirability to Control Scale (Burger \& Cooper, 1979) were examined in order to draw items for the new psychological empowerment scale. The SPCS was selected as the primary scale from which items would be drawn because it is the most commonly used and empirically validated measure of intrapersonal empowerment within the Community Psychology field. Two items were adapted from the SPCS (Zimmerman \& Zahniser, 1991) in order to measure motivation to control. 
The original SPCS item, "I would prefer to be a leader rather than a follower," was selected because it consistently loads highly onto the Leadership Competence subscale of this intrapersonal psychological empowerment measure (Peterson et al., 2006; Zimmerman \& Zahniser, 1991). It was adapted, however, to tap into general motivation to control by adding "In general," to the beginning of the item (i.e., "In general, I would prefer to be a leader rather than a follower").

In contrast to the positively worded item, which tapped into general motivation to control, the negatively worded item was adapted from the SPCS to measure motivation to control within the three OYA settings (i.e., living unit, treatment, school/vocation). The original item, "I would rather someone else took over the leadership role when I'm involved in a group project," was selected because it was the highest loading, negatively worded item onto Zimmerman and Zahniser's Leadership Competence sub-sale. This item was adapted to be more concise and use simpler language. The final item read, "When I work on group projects, I prefer to 'take a back seat."”

Seven different scales that, in part, measured perceived competence were examined in order to draw items for this domain (Bobak, Pikhart, Rose, Hertzman, \& Marmot, 2000; Holden et al., 2004; Israel et al. 1994; Lachman \& Weaver, 1998; Menon, 1999; Paulhus, 1983; Spittal, Siegert, McClure, \& Walkey, 2002). One item from the Perceived Constraints Scale (PCS; Lachman \& Weaver, 1998) and one item from the Empowerment Scale (Menon, 1999) were selected to measure perceived competence. In general, these two scales sampled from populations that were most similar to the current study's population, utilized a similar survey format, and were easily adapted to the 
correctional context. Menon's Empowerment scale was one of the only scales examined that conducted a factor analysis on its items.

The positively worded item was based on Menon's (1999) item measuring perceive competence in employed business students ("I can influence decisions taken in my department"). This item was selected because of its high factor loading and because it is aimed at measuring control over decisions. It was adapted to fit the correctional context by eliminating the phrase related to employment. The final item, which was more concise, read, "I can influence decisions made."

The negatively worded item measuring perceived control was adapted from the PCS by Lachman and Weaver (1998). This item was selected because it was the best reverse worded item available, fitting the context as well as reflecting the positively worded item selected. The order of words within the original item ("There is little I can do to change many of the important things in my life") was changed to read, "There is little I can do to change many of the things important to me," because the item did not flow well overall without a reference to the context within the item.

Overall, item selections and adaptations for the Intrapersonal component of psychological empowerment were based on criteria discussed above as well as committee feedback. Refer to Table 1 for the final items and their abbreviations and Appendix A for a copy of the entire survey, including the PE scale, administered to the youth.

Interactional Psychological Empowerment. Three domains were chosen to represent the Interactional component of psychological empowerment. The domains included awareness of resources, critical awareness (i.e., environmental/circumstantial reflection), and problem-solving. These domains were chosen because they underscore 
the interactional component of psychological empowerment (Zimmerman, 1995; Zimmerman \& Warschausky, 1998).

Only one scale (Empowerment Scale for Youth in Tobacco-Related Intervention; Holden et al., 2004; Holden et al., 2005) was found to explicitly measure awareness of resources. Therefore, the two items measuring this domain of interactional psychological empowerment were developed based on this measure. The item aimed at measuring awareness of resources within Holden and colleagues' $(2004 ; 2005)$ measure was framed as question ("What resources are available to your group in your community or school to help you work on tobacco issues?") rather than a statement. For this reason, the question was revised to fit the survey format (i.e., items as statements). Within the statement, examples were provided to help youth understand what was meant by "resources." The following is the positively worded item developed to measure awareness of resources based on Holden and colleagues' work: "I know where to go to get information about (my progress on my treatment goals; taking classes outside of OYA; starting a new activity like a basketball tournament)." Only one of the above examples was provided, depending on the OYA setting that the youth were asked to think about while responding to the items.

The negatively worded item measuring awareness of resources was also developed based on Holden and colleagues' $(2004 ; 2005)$ survey question because no other survey items were available. The negatively worded item developed to measure this domain read, "If I have a major problem with an OYA staff member, like (I disagree with something she or he asked me to work on; he or she says mean or rude things about my work; or he or she makes fun of me and it hurts my feelings), I am unable to solve it," 
and utilized different examples representing different situations in which resources could be sought. Only one of the three examples was provided per OYA setting.

Two scales (Social Worker Empowerment Scale developed by Frans [1993] and the Cognitive, Emotional, and Behavioral Empowerment Scale developed by Speer and Peterson, [2000]) were examined in order to develop items measuring critical awareness. These were the only two scales found that attempt to measure critical awareness. While Frans' (1993) scale measures environmental critical awareness, Speer and Peterson's (2000) scale assesses critical awareness related to power. The positively worded item measuring critical awareness was adapted from an empowerment scale (Frans, 1993), and the associated reverse worded item was developed based on this scale. Although Speer and Peterson's scale has been used to measure critical awareness and psychological empowerment more broadly in other work (Peterson et al., 2005), Frans' measure was the only scale that measured critical awareness in a way that was not so context-dependent as to inhibit adaptation of the items.

For this reason, the positively and negatively worded items assessing critical awareness were developed based on Frans' (1993) original item that read, "I am usually able to think through all the issues." This item, however, was found to be too general. It was suggested by the Dissertation Committee that items measuring critical awareness be more specific by asking about things that impact youths' behavior. In doing so, it was suggested that the different referents (e.g., peers, staff) be used within the positively and negatively worded items. The final positively worded item developed to measure this domain referred to other youth as a potential influence on behavior ("The beliefs of other youth at OYA make it difficult to do what is right"). In contrast, the negatively worded 
item used staff as the potentially influential referent ("OYA staff have little influence on my behavior"').

Finally, two items assessing problem-solving were adapted from an inventory of problem solving behaviors (Heppner \& Peterson, 1983). Of the two scales assessed, the Personal Problem-Solving Inventory (PPSI; Heppner \& Peterson, 1983) was used for item selection because it was more commonly used in the literature on problem-solving compared to that developed by Maydeau-Olivares and D'Zurilla (1996). Additionally, Heppner and Peterson's measure included both positively and negatively stemmed items.

The original positively worded item from the PPSI ("When making a decision, I weigh the consequences of each alternative and compare them against each other;" Heppner \& Peterson, 1983) was chosen because it was the highest loading item and because it fit the context of the present research. The only adaptation made to this item was to change "alternative" to "choices" in order to simplify the language. The final positively worded item measuring problem-solving read, "When making a decision, I weigh the consequences of each choice and compare them against each other."

The negatively worded item assessing problem-solving was also drawn from an item within the PPSI ("When confronted with a problem, I tend to do the first thing that I can think of to solve it"). This item was the highest loading negatively worded item within the problem-solving sub-scale. No adaptations or revisions were made to this item.

Overall, the selected or adapted items measuring the Interactional component of psychological empowerment were based on criteria discussed above as well as committee 
feedback. Refer to Table 1 for the final items and their abbreviations and Appendix A for a copy of the entire survey administered to the youth.

Behavioral Psychological Empowerment. Finally, three domains were chosen to assess the Behavioral component of psychological empowerment. These selections were made based on the criteria previously outlined as well as conceptualizations of psychological empowerment discussed by Zimmerman (1995) and his colleagues (Zimmerman \& Warschausky, 1998). The domains included advocacy, involvement, and coping behavior. Similar to the item selection process conducted for the other two components of psychological empowerment, two items were selected to measure each of the domains of Behavioral psychological empowerment. One item in each pair was positively and the other negatively worded.

Although two scales were examined for items measuring advocacy, the Psychological Empowerment Scale (PES) established by Akey and colleagues (2000) was used to develop items for the present scale. Akey and colleagues' PES was used for item selection instead of the Cognitive, Emotional, and Behavioral Psychological Empowerment Scale (Speer \& Peterson, 2000) because its items were more readily adapted to the correctional facility context.

Akey and colleagues' (2000) highest loading item on the Formal Participation sub-scale ("I would be likely to speak out about an important policy issue concerning families") was used as a foundation for measuring advocacy. This item was subsequently revised in order to simplify the flow of the item. The final positively worded item that assessed advocacy read, "I speak up about issues important to me." 
There were no negatively worded items within the empowerment scales examined that adequately assessed this advocacy. Therefore, the negatively worded item assessing advocacy was developed based on several empowerment scales, including Akey and colleagues' (2000) and Speer and Peterson's (2000). The negatively worded item developed based broadly on other scales reflected an individual's tendency to assert oneself in matters that are regarded as important ("I let things go rather than to speak up about them, even if they are important to me").

Involvement was emphasized by Zimmerman (1995) and colleagues (Zimmerman \& Warschausky, 1998) as an important aspect of Behavioral psychological empowerment; however, no scales adequately assessing involvement were found. For this reason, two items were developed to measure active involvement or participation in activities in each of the three OYA settings based on the researchers understanding of this domain. The positively worded item developed to measure involvement was designed to be context appropriate and well constructed. It read, "I actively participate in activities, even if I don't have to." The negatively worded item assessing this domain ("If given the choice, I prefer to do other things rather than participate in activities") was developed to inversely reflect the positively worded item.

Five scales were examined prior to item selection for the coping behavior domain of interactional psychological empowerment (Akey et al., 2000; Amirkhan, 1990; Ayers, Sandler, West, \& Roosa, 1996; Conor-Smith, Compas, Wadsworth, Thompsen, \& Saltzman, 2000; Patterson \& McCubbin, 1987). Akey and colleagues' (2000) measure was used for item selection because its items could be applied to the correctional context, regardless of the situation or problem, with only minor revisions. 
The positively worded item assessing coping behavior was also adapted from Akey and colleagues' (2000) item that read, "There is at least one other parent I can go to for emotional support." Although this item pertains to the Informal Participation subscale, it is also related to coping. This item was chosen because it was the highest loading item on Informal Participation sub-scale related to coping. The original scale was written for parents with children with disabilities. This item asked about peer-topeer support, which is why "parent" was changed to "youth." The final item measuring coping behavior in incarcerated youth read, "There is at least one other youth I can go to for support."

The reverse worded item was developed based on the positively worded item from Akey and colleagues' (2000) scale, which did not include negatively worded items. The item was intended to behaviors related to isolation and lack of support. This item read, "I only have myself to rely on for support."

The six items underlying the Behavioral component of psychological empowerment were selected, adapted, or created based on prescribed criteria and committee feedback. Refer to Table 1 for the final items and their abbreviations and Appendix A for a copy of the entire survey administered to the youth.

Instructions. A set of instructions was developed to accompany the scale items within each OYA setting. The instructions for each scale were based on instructions provided to the youth on previously administered instruments during the OYA Annual Survey. The instructions explicitly directed youth participants to think about a specific setting within OYA (e.g., living unit, treatment, school/vocational training) and provided a timeframe (i.e., the last month) within which their responses should correspond. 
Directions related to OYA setting and timeframe were in bold typeface, and the OYA setting was mentioned twice within the instructional set.

Youth Advisory Committee. In an effort to include the perspective of incarcerated youth in the scale development process, the Youth Advisory Committee (YAC) provided feedback on two early drafts of the psychological empowerment scale. The YAC is composed of approximately 16 youth residing under OYA custody. Their input was sought on two different occasions. These meetings were semi-structured, where youth took time to read over and respond to each item within the scale. Only one scale (i.e., referring to the living unit) was provided to youth for feedback. An informal conversation followed regarding youths' general thoughts about the measure as well as specific questions related to the items. For example, during the first meeting with the YAC, one item used a time referent equal to one year (i.e., "in the past year...”). Youth feedback lead to this item being changed from one year, which was much too long, to one month. Another example lead to the elimination of an item that was perceived to be identical to the youth because they did not distinguish between "understanding" and "dealing with" service systems.

The researcher also posed questions to the YAC about examples provided within specific items and about language used (e.g., language that could be perceived as too "flowery" for young men). Of particular concern to the researcher was that some items might use language or refer to the youth inappropriately. For example, one item directly referred to the young men as "incarcerated youth," which explicitly directed the youths' attention to the fact that they are incarcerated. Although the researcher was concerned about the youths' response to this item, the YAC assured the researcher that this term is 
commonly used and accepted amongst the OYA population of young men. The item wording was, thus, maintained. Other language, which had the potential to seem "girly" to the youth (e.g., "emotional support"), was also approved by the YAC.

Although early drafts of the psychological empowerment scale constructed for use in this research did not reflect items selected following the processes discussed above, input from the YAC regarding definitions of key terms, language and examples used, as well as overall understanding of items developed in the early phases of development was considered when finalizing the scale. For example, during an early meeting with the YAC, the group of young men brainstormed a list of potential examples that the researcher could use in an item related to Behavioral PE. The researcher wanted to ensure that the example used in the item was: (1) an activity that youth were interested in doing and (2) an activity that youth would be willing to advocate for. One of the ideas mentioned by the YAC was a basketball tournament. Again, although the original item that this example was generated for does not appear in the current version of the survey, the researcher used this example to expand an item in the final version of the scale ("I know where to go to get information about starting a new activity like a basketball tournament"). In addition to the goal of including youths' perspectives, referencing previous YAC feedback was done to augment face validity and cultural-sensitivity of survey items.

\section{Conclusion}

Through a stringent literature review process, scales and potential items were selected to measure one of nine domains of psychological empowerment within one of three of its principle components (i.e. Intrapersonal, Interactional, Behavioral). After 
scale and possible item identification, specific criteria (e.g., factor loadings, language, valence, contextual fit) were assessed to select the final items. Committee feedback was then used to adapt the items and YAC feedback was referenced in order to optimize face validity and language used within the scale. The final versions of the scales, which each included 18 items, are available in Appendix A. 


\section{CHAPTER V}

\section{Study Context}

This study was developed within a youth correctional context at the Oregon Youth Authority (OYA). While OYA is one of many state agencies representing the juvenile justice system across the country, distinctions between OYA and other juvenile justice systems can be made. These distinctions will be explored within this chapter. Due to the specificity and uniqueness of this particular setting, connections between empowerment and OYA will also be described within this chapter. OYA provides unique programming for youth, which has yet to be studied within the empowerment literature. Therefore, this chapter will highlight the ways in which empowerment might manifest within OYA. Justification for studying psychological empowerment within three settings at OYA will also be presented. Before doing so, a more thorough discussion of OYA and its programming will be provided.

\section{Oregon Youth Authority}

Oregon Youth Authority (OYA) is the State agency responsible for the supervision and care of youth who enter the juvenile justice system in Oregon. OYA oversees both probation (i.e., residential care, foster care, or home care) and correctional facility (i.e., public facility or community services) commitments. The focus of the current study was placed on facility services, both correctional- and re-entry-oriented. Refer to Figure 1 for a visual description of the process through which youth enter and exit the OYA system.

OYA's mission is "to protect the public and reduce crime by holding youth offenders accountable and providing opportunities for reformation in safe environments" 
(Oregon Youth Authority, 2012b). It maintains four core values: (1) professionalism; (2) accountability; (3) integrity; and (4) respect. OYA's overarching vision is to guide youth to lead productive, non-criminal lives in the community (Oregon Youth Authority, 2012b).

OYA manages a total of ten correctional and re-entry facilities across the state of Oregon. Only one of its facilities is dedicated to the treatment and rehabilitation of female offenders. As there was not a sufficient number of female offenders to constitute a separate sub-group upon which analyses could be conducted, this study focused exclusively on young men. The nine OYA facilities that house young men are budgeted to serve up to 716 total youth. Within each facility, youth reside in living units or cottages with approximately 24 other young men. Refer to Table 2 and to the sections below for detailed information about each of the nine facilities included in the present study.

Youth who enter OYA correctional and re-entry facilities are typically between the ages of 12 and 17, and can be mandated to remain in OYA facilities up to the age of 25. Youth residing within OYA facilities have been sentenced either through OYA or through Department of Corrections (DOC). Youth sentenced within OYA do not have a specific length of sentence to serve (i.e., indeterminate sentencing) and transition out of OYA as a result of progress through treatment programming. DOC youth are convicted of crimes under Oregon Ballot Measure 11. This measure established minimum sentencing for a number of crimes including first and second degree robbery, several crimes relating to sexual assault, first and second degree assault or manslaughter, and 
murder. Youth convicted under DOC jurisdiction are required to serve out a sentence of specific duration (i.e., determinate sentencing).

Within the first 30 days of contact with OYA, youth are given a Risk Needs Assessment (RNA). The RNA examines each youth's criminal risks and needs based on 11 domains or skill areas established by OYA. These domains include: (1) substance abuse; (2) mental health; (3) education/school; (4) use of free time; (5) family/parenting; (6) interpersonal relationships; (7) criminal/delinquency history; (8) employment; (9) attitudes and beliefs; (10) aggression; and (11) social skills (Oregon Youth Authority, 2012b). Results from the RNA are used to inform the development and implementation of the youth's case plan. In connection with domains relevant to each youth, several short- and long-term goals, known as competencies, are determined. The case plan is used to help the multi-disciplinary team (MDT) determine appropriate reformation and treatment services as well as educational and vocational training to help youth achieve their goals.

OYA offers a number of curricula to help youth achieve their goals. These curricula utilize cognitive-behavioral and social learning principals to address mental health, criminological, behavioral, psycho-social, and other social skills issues. Currently, OYA offers 12 core curricula addressing these issues, including: (1) "What Got Me Here?;” (2) “Changing Offender Behavior \#1 and \#2;” (3) "Skill Streaming;" (4) “Core AOD Treatment;" (5) "Social Skills/Boys Town;” (6) "Coping with Depression;" (7) “Dialectical Behavior Treatment;" (8) “Core Sex Offender Treatment;" (9) "Street Smarts;" (10) "Seeking Safety;" (11) "Pathways to Self-Discovery;” and (12) “Aggression Replacement Training” (Oregon Youth Authority, 2012b). The overarching 
goal of these services is to decrease risk factors and strengthen positive, pro-social skills (Oregon Youth Authority, 2012b).

In addition to the aforementioned reformation and treatment services, OYA offers both educational and vocational opportunities to youth. OYA has a contract with the Oregon Department of Education to provide educational services comparable to those offered in public schools (Oregon Youth Authority, 2012b). OYA also offers youth the chance to improve their knowledge, skills, and abilities in a variety of trade areas.

Although specific vocational or work experiences offered depend on the facility in which a youth resides, each facility provides a minimum of three and a maximum of 18 vocational activities. These include such jobs as a carpenter's assistant, a food service worker, an electrician's assistant, and a waste water technician. Complementing workrelated vocational opportunities, other programming, such as Project Pooch (i.e., dog training), present chances to participate in extracurricular activities. For youth residing within one of the three re-entry facilities, there are opportunities for employment within the surrounding community on a work crew, in a job shadow, or in a quasi-internship.

OYA has made an effort to be sensitive to the cultural background of youth living within its facilities and utilizing its services. It offers culturally sensitive programming, including minority youth transition programming, gang intervention programming (i.e., Street SMARTS), and other culturally specific and multi-cultural support groups (Oregon Youth Authority, 2012b). Special events, such as an assembly for Black History Month, a celebration for Cinco de Mayo, and Native American pow-wows, on or during cultural holidays are also provided (Oregon Youth Authority, 2012b). Additionally, the Office of Minority Services at OYA maintains a number of collaborative partnerships around the 
State of Oregon in order to better serve minority youth (Oregon Youth Authority, 2012b). Accommodating youth and families where English is not the primary language spoken, translation and interpretation services are provided when necessary (Oregon Youth Authority, 2012b).

After receiving the appropriate treatment, and for DOC youth who have completed their sentence, youth are released from the correctional facilities. Prior to release, some youth, both under OYA and DOC jurisdictions, have the opportunity to participate in transition-specific programming in the re-entry facilities. Within transition facilities, they have additional vocational or work experiences within the community. All youth, no matter their participation in transition-specific programming, collaborate with their assigned MDT to determine post-release placement. When released from a correctional or a re-entry facility, youth will return home, live on their own, or go to a community-based treatment center. At this point, youth work with their communitybased parole officers to satisfy any remaining requirements or stipulations of their supervision.

OYA Facilities. As previously mentioned, there are nine total facilities at OYA that house young men across the State of Oregon. Youth residing within correctional and transition facilities were included in this study. Descriptions of each type of facility (i.e., correctional or transition) follow. Refer to Table 2 for more detailed information about age, ethnicity, most serious crime committed, and other background information on youth residing at each facility.

Correctional Facilities. There are a total of 6 correctional facilities run by OYA. They typically house between approximately 50 and 190 young men. Within the 
correctional facilities, youth receive crime-specific treatment (e.g., for sexual offending) as well as other behavioral and social-emotional treatment as required by the RNA. Some facilities offer specific types of programming, such as substance abuse treatment and treatment for sexual offending. Other facility-specific functions include intake and special activities (e.g., Project Pooch, a program that provides youth the opportunity to train and care for shelter dogs). Youth under OYA and DOC jurisdiction reside within these facilities.

Transition Facilities. OYA oversees a total of three transition facilities across the state of Oregon. At the transition facilities, youth make final arrangements for release and gain additional life and social skills for future reintegration success. Through special programming provided at these facilities, youth have the unique opportunity to work in the community on work crew, job-shadowing, or quasi-internships. The environment within the transition facilities tends to be less rigid than it is within correctional facilities, allowing youth more autonomy and providing them with more responsibilities. For example, one transition facility is not enclosed by a fence, possibly providing youth with a greater sense of freedom. This shift in programming and physical environment is to encourage youth to continue strengthening skills needed upon return to the community (e.g., responsibility, self-control, self-regulation). The transition facilities are generally small compared to other correctional facilities, housing between approximately 25 and 50 young men. With the exception of one transition facility, which typically houses only sex offenders convicted under OYA jurisdiction, youth from OYA and DOC court jurisdictions can reside within the same transition facilities.

Distinctions between OYA and the General Juvenile Justice System 
OYA is nested within the broader juvenile justice system. While the juvenile justice system within Oregon reflects some national trends, Oregon stands out as one of the leading state agencies in the country for their adoption of a strengths-based perspective. One national trend that Oregon follows is a high incarceration rate. In fact, in 2010, Oregon was estimated as having the third highest youth incarceration rate in the country (Office of Juvenile Justice and Delinquency Prevention, 2011). Despite reflecting this trend, OYA remains largely independent of arrests and referral benchmarks (Oregon Youth Authority, 2012a). Officials and court systems outside of OYA typically make arrests and referrals. Policies within OYA do not always align with state and national arrest and sentencing patterns that can be characterized as punitive. Once youth enter OYA correctional facilities, they are provided treatment and other services that are aimed at holding them accountable for crimes committed as well as providing them with opportunities for reformation (Oregon Youth Authority, 2012b). Currently there is a growing commitment within OYA to offer opportunities for positive development while incarcerated. In 2012, OYA published a brief on its increasingly popular approach to the treatment of youth. This perspective is strengths-based, viewing youth as a resource as opposed to a victim or a villain. From this standpoint, youth are considered to be similar, in many ways, to non-incarcerated adolescents who are capable of engaging in pro-social behaviors and contributing to society (Oregon Youth Authority, 2012c). Through this lens, pro-social attitudes and behaviors are cultivated and youth strengths are developed in addition to efforts directed toward risk reduction, reformation, and retribution programming (Oregon Youth Authority, 2012c). 
OYA is just beginning to formally promote this strengths-based perspective from the top down, but positive outcomes of strengths-based treatment and services (e.g., skills, competencies) that are currently in place are already being measured (Peters \& Myrick, 2011). One potentially positive outcome of strengths-based programming that is not currently measured is psychological empowerment. Active participation in programs that foster positive development might elicit a sense of psychological empowerment. As a result of the experience of psychological empowerment within OYA programming, it is possible that additional constructive behaviors might surface. A more detailed discussion of the potential utility of empowerment in correctional facilities, and specifically within OYA, follows.

Empowerment with Incarcerated Youth

As discussed in Chapter III, research on youth empowerment programs have focused on "at-risk" youth, particularly minorities. It would seem a small extension of this work to conduct research of this nature with incarcerated youth, who are often also considered "at-risk" once they re-enter the community. Research on the antecedents, correlates, and impacts of youth empowerment would benefit from including important intervention work such as programming for incarcerated youth. While this might be an interesting population within which to study empowerment, to date, no research has been conducted on this construct within youth correctional facilities.

Despite the absence of research, empowerment might be of particular importance to incarcerated youth. Youth find themselves incarcerated, in part, as a result of environmental circumstances that might leave them with deficient socials skills, low or unstable self-esteem or confidence, and feelings of powerless (Baumeister, Bushman, \& 
Campbell, 2000; Donnellan, Trzesniewski, Robins, Moffit, \& Caspi, 2005; Marshall, 1989; Page, 1991). For example, a youth living in poverty with little social or economic resources may be enticed to join a gang for social support or engage in criminal activities in order to make money or satisfy his basic needs. The incarcerated atmosphere oftentimes does little to improve these circumstances. During incarceration, youth have little control over their lives (Schwartz, 2000). The typical atmosphere of State correctional facilities has been characterized as rigid (Schwartz, 2000), and treatment is often focused on reducing problem behavior rather than cultivating strengths (Schwartz, 2000; Zeldin, 2004). A strengths-based approach, such as the one being implemented by OYA, might result in increased self-esteem, confidence, and greater perceived control of one's life, which are all characteristics of psychologically empowered individuals.

At a time of significant individual and environmental change, these cognitive developments might help incarcerated youth succeed when re-entering the community. According to leading researchers and practitioners in the youth transition-to-community field, incarcerated youth need the opportunity to develop and demonstrate psychosocial skills and abilities prior to release (Abrams, 2006; Altschuler \& Brash, 2004; Steinberg et al., 2004). Developmentally appropriate skills include, among other things, mastery, competence, and autonomy or independence (Altschuler \& Brash, 2004; Steinberg et al., 2004), all of which are elements of psychological empowerment.

Psychological empowerment may be a critical skill for incarcerated youth to develop prior to their release. Psychological empowerment offers the potential to strengthen youths' abilities to reflect on the situation at hand, which, at the time of release, may be extremely difficult due to dual transitions and other barriers to success 
(Abrams, 2006; Altschuler \& Brash, 2004). It might also help incarcerated youth to feel competent and confident in their ability to create change either within themselves or within the community at large. Through the experience of psychological empowerment, youth may be better able to work positively and pro-socially with others to produce that change. Youth are often in a very different developmental stage when they return to the community as compared to when they were first incarcerated (Glick \& Sturgeon, 1998), especially youth who committed a crime several years prior to their release. For some, a more pro-social and healthy future is both anticipated and desired (Abrams, 2006). It is only fair to these youth that they are given the opportunity to cultivate the aspects of psychological empowerment (e.g., competence, confidence, and autonomy) that are required to foster successful community re-entry.

Although empowerment might have a positive impact on short- and long-term behavior as well as community re-integration, empowerment with incarcerated youth could be considered controversial. From one perspective, these youth have already demonstrated an abuse of power in one aspect of their life, resulting in incarceration. Incarcerated youth have committed at least one crime, taking advantage of or victimizing another person in some fashion. Victim advocates might argue that empowering "perpetrators," particularly those that have committed a violent or sexual act against another person, may be particularly counterintuitive.

Most incarcerated youth will, at some point in their lives, re-enter society. For this reason, the goal of programming within correctional facilities like OYA is to ensure that these youth are productive and safe upon release. Enhancing empowerment may position youth to maximize their skills to create a positive life upon re-entering the 
community. As many of these young men experience a lack of power in several important aspects of their lives (e.g., daily routine, career development; Schwartz, 2000), services offered in correctional facilities during their incarceration should assist youth in developing cognitive and behavioral strengths associated with empowerment. In doing so, dominant narratives of taking power or control over others will not be perpetuated. Instead, the process of empowerment would demand that a youth reflect upon the social, political, and economic factors that impact his life path and focus on strengths he can utilize to lead a more pro-social life.

Empowerment, Positive Youth Development, and the Juvenile Justice System

At the same time that youth empowerment models were developing, a similar approach, known as positive youth development (PYD), was also growing in popularity. PYD and empowerment have many commonalities, including a strengths-based perspective, environmental and opportunity structures, and the recognition of interactions between persons and their environment. As a result of these similarities, programming that incorporates one or both of these perspectives could offer unique advantages to helping youth succeed within correctional facilities and within the community.

PYD originated in the late 1980s and 1990s, and takes a strengths-based approach to helping youth transition from childhood to adulthood (Zeldin, 2000). It emphasizes the promotion and cultivation of strengths within individuals as well as the development of protective factors that help youth succeed (Catalano, Hawkins, Berglund, Pollard, \& Arthur, 2002; Zeldin, 2000; Zeldin \& Price, 1995; Lerner, Almerigi, Theokas, \& Lerner 2005; Roth \& Brooks-Gunn, 2003). Foundational to the PYD approach is the notion that an emphasis on the reduction of behaviors does not address the full spectrum of resources 
that youth need for healthy development. In fact, a commonly cited quotation within the PYD literature reads, "problem-free is not fully prepared" (Pittman, Irby, Tolman, Yohalem, \& Ferber, 2003, p. 6). In his introduction to a special issue of Applied Developmental Science on PYD, Zeldin (2000) summarized characteristics of PYD programs across the literature. These characteristics included: (1) youth empowerment; (2) exploration; (3) competence and mastery; (4) emotional health; (5) compassion and generosity; (6) community connections and belonging; and (7) civic participation (Zeldin, 2000). PYD has also been characterized as integrating "the 5 C's," which include: (1) competence; (2) confidence; (3) character; (4) connection; and (5) caring (Roth \& Brooks-Gunn, 2003). In doing so, it takes a developmental approach to building strengths (e.g., competence and confidence). Strengths are cultivated through the provision of opportunities (e.g., self-directed learning, participation in adult roles) and supports (e.g., emotional, strategic, motivational) by positive influencing adults (Zeldin \& Price, 1995). Through the promotion of strengths in youth, the underlying goal of PYD is to jointly decrease risk behaviors and increase successful, healthy, pro-social development.

A relatively recent movement within the juvenile justice system has pushed for a paradigm shift in youth intervention programming. This shift moves away from the exclusive use of deficits-based treatment and incorporates strengths-based approaches, such as PYD, into programming. Several researchers and practitioners have underscored the utility of PYD within the juvenile justice system (Bazemore \& Terry, 1997; Butts et al., 2005; Schwartz, 2000). One of the original uses of PYD was to address crimes committed by youth prior to their arrest (Bazemore \& Terry, 1997). For this reason, 
researchers and practitioners believe that PYD can benefit youth while incarcerated as well. Butts and colleagues (2005) argue that there is little evidence-based treatment for the majority of incarcerated youth, especially those that committed a non-violent crime (e.g., property offense, minor drug offense, misdemeanors) or are not considered “extreme cases." They suggest that PYD can be useful to the majority of incarcerated youth who have offended for reasons other than psychological problems, such as social (e.g., negative peer association), political (e.g., status, defiance of authority), or economic (e.g., poverty) reasons. Butts and colleagues (2005) also believe that PYD is a useful intervention tool for incarcerated youth because it acknowledges the less-than-ideal environments within which many delinquent youth grow up. By assessing the strengths that youth have at intake, tailoring a program of treatment and intervention that builds on their strengths, and offering new opportunities for participation and skill development, PYD can help incarcerated youth create a pathway for future success (Butts et al., 2005). Even as the theoretical underpinnings of PYD have gained more support within the field, the potential benefits of this type or of similar programming, such as empowerment, have not yet been studied empirically.

Empowerment might have similar benefits within youth correctional interventions because PYD and empowerment have much in common. Most obviously, they both emphasize the importance of personal strengths such as competence, confidence, and mastery. Both empowerment and PYD encourage youth to take on new roles (e.g., leadership) and actively engage in community activities. The characteristics of these settings are also similar in that they both include a supportive, welcoming atmosphere that cultivates positive, collaborative relationships between youth and adults and among 
youth, that allows youth the space for decision-making and leadership development, and have expectations for positive behavior that youth must satisfy (Cargo et al., 2003;

Jennings et al., 2006; Roth \& Brooks-Gunn, 2003). Less obvious commonalities include the recognition that context is important and that the nature of reality is shaped by person-environment interactions. Empowerment and PYD also take a similar approach to prevention, emphasizing the promotion of health and well-being, in addition to the reduction of risk. In fact, both empowerment and PYD originated, in part, from Community Psychology's critique of traditional prevention methods (i.e., deficits-based; Lerner et al., 2005). Due to the similarities between empowerment and PYD, an examination of the utility and outcomes of empowerment within programs where PYD has been used might be justified.

Although OYA has not specifically adopted an empowerment approach with treatment and other service programming, the agency has formally advocated for PYD to become a mainstay of OYA culture (Oregon Youth Authority, 2012c). Its programming also reflects many of the strengths-based characteristics inherent to PYD. As a result of the similarities between PYD and empowerment, OYA's services might evoke a sense of empowerment within youth in its correction facilities.

Empowerment and Youth at $O Y A$

Although not specifically characterized as empowerment programming, OYA's strengths-based or PYD goals related to treatment and other services align well with empowerment. One of the principal philosophies within OYA facilities is to encourage youth to demonstrate the skills they learn in order to deal with difficult situations in school, work, or their social life. This is designed to encourage youth to take control and 
feel efficacious about their pro-social behavioral changes (D. Martin \& T. Bendt, personal communication, September 7, 2011). Through programming, youth should also learn life skills that help them feel more confident, competent, and in greater control of their lives before they begin the community re-integration process (B. Blisard, personal communication, September 29, 2011; D. Martin \& T. Bendt, personal communication, September 7, 2011). Control, self-efficacy, competence, and other aforementioned qualities are characteristic of psychological empowerment.

Through OYA programming, there are several ways in which youth can develop a sense of psychological empowerment. OYA offers many different services that, together, help youth achieve short-term, intermediate, and long-term goals. Working backwards through the theory of change at OYA in Figure 2, OYA seeks to help youth develop the ability to lead productive, "non-criminal" lifestyles in their community upon release from their custody (Oregon Youth Authority, 2012b). Youth are better able to lead this type of lifestyle if they gain a number of positive, pro-social skills as well as learn how to decrease or address risk factors surrounding them while they are incarcerated. To do this, OYA has established several skill areas or domains (e.g., education, vocation, life/social skills, offense-specific, and mental health) to help youth achieve these goals in different aspects of their life. Within these domains, each youth is assigned several short-term goals known as competencies. These goals range from willingness to learn (educational domain) to pro-social engagement with others (life/social skills domain) to motivation to change (offense-specific).

To help youth achieve these goals, OYA offers several treatment-oriented (e.g., "What Got Me Here?," Pathways to Self-Discovery), educational (i.e., high school 
courses), vocational (i.e., youth work programs), and other programming or service opportunities (e.g., culturally-specific support groups). As depicted in theory of change model for OYA in Figure 2, the processes that youth experience during these activities have the potential to be empowering and thus may result in a greater sense of psychological empowerment. For example, a recently incarcerated youth may be involved in the treatment program known as "What Got Me Here?," which teaches youth about cognitive skill building (Oregon Youth Authority, 2012b). Within this treatment program, youth learn to reframe their risky thoughts and model pro-social behavior for one another (Oregon Youth Authority, 2012b). In doing so, they can address competencies related to the life/social skills domain, the mental health domain, as well as the offense-specific domain. These processes also have the potential be to empowering for the youth. To the extent that youth are actively engaged in the treatment program, they may receive positive feedback from staff about successful reframing or pro-social behavior, which will increase their self-efficacy and sense of competence. They may find that they are able to take on new leadership roles by modeling positive behavior for other youth. Additionally, group work aimed at positive behavior change may help youth learn to work with others in an effective manner, such as discussing important issues or making positive decisions.

Another instance where youth may become empowered through treatment programming is during "Pathways to Self-Discovery." This treatment program allows youth to decide what, within himself, requires cognitive change, encourages him to apply tools for cognitive change, and then to take control of that change within the program and within other settings (e.g., school, the living unit; Oregon Youth Authority, 2012b). By 
providing the necessary tools to a youth, yet allowing him to create the change that he believes is necessary, the youth may gain a greater sense of control as well as actual control over his treatment plan, resulting in a state of psychological empowerment.

Youth may also experience psychological empowerment in programming other than treatment. For example, youth have the opportunity to participate in work programs, which teach job-related skills (Oregon Youth Authority, 2012b). Through these activities, youth have the opportunity to take on new roles and responsibilities within the facility or even within the community as well as demonstrate their competence in productive, pro-social tasks. In doing so, they may experience psychological empowerment.

\section{Selection of OYA Settings}

Research on psychological empowerment has not been conducted in the context of youth correctional facilities. An investigation of this important construct in this environment is justified because of its possible benefits to incarcerated youth. Exploring psychological empowerment in multiple settings within youth correctional facilities may be most advantageous given that the experience of empowerment is context dependent (Perkins \& Zimmerman, 1995; Rappaport, 1981; Rappaport, 1987).

Within the present study, psychological empowerment was measured in three different settings within OYA: (1) youth's living unit; (2) youth's treatment groups; and (3) youth's school or vocational activities. There were two important reasons for studying psychological empowerment in different settings. First, it was recognized that the extent to which psychological empowerment is experienced depends largely on context (Rappaport, 1981; 1987). Studying psychological empowerment within three 
contexts prevents the conflation of its experience within distinct OYA settings. It also allows youth to report experiencing psychological empowerment differentially. Within the living unit setting, for example, youth might have very different goals related to personal and environmental change compared to school or vocational activities. Goals in the living unit might include starting basketball tournament or lobbying to increase internet access. A youth looking to gain more work experience might have the desire to establish a relationship with a specific community organization. In addition to potentially distinct interests, factors impacting the achievement of their goals or desired changes might also differ across settings. Goal achievement within each setting depends upon, among other factors, staff, rules and regulations, and norms and values. These factors are likely to vary upon setting.

Second, different OYA settings were studied in order to contribute to the validation of the newly created measure. By measuring psychological empowerment in three different settings, the researcher was able to compare its operationalization across different contexts within a broader system. It was assumed that the conceptualization, and thus operationalization, of psychological empowerment was the same across the three settings. This assumption was made because all settings were nested within the same institution. The larger context, OYA, was thought to similarly shape all three settings but programming and youth-staff interactions would impact the experience of psychological empowerment. For this these reasons, it was anticipated that the conceptualization of psychological empowerment would be the same, but the degree to which youth experience it within the three settings would be different. 
Research on empowerment supports the notion that systemic factors are influential to its experience. In their investigation of empowerment of students and parents at an alternative school, Gruber and Trickett (1987) found that the hierarchical culture of the school district limited the extent to which students and parents experienced empowerment. LeRoy and her colleagues (2004) also discovered that beliefs about smoking in the teen community and tobacco prevention funding impacted the extent to which youth reported feeling empowered in a tobacco intervention program. Even in intentional programming that centers on empowerment, aspects of the larger environment (e.g., culture, values, politics) can inhibit empowerment at lower levels of a system. At OYA, it was presumed that institutional-, facility-, unit-, and setting-level factors would shape the degree to which youth experienced psychological empowerment. While it is evident that an institution impacts individual-level empowerment, no evidence was found that gave reason to believe that the conceptualization of empowerment would be different among settings within a single organization or program.

The living unit, treatment, and school or vocational settings at OYA were chosen as the areas of focus for reasons related to sample size, overlapping activities, and research supporting community re-entry outcomes. The large majority of, if not all, youth participate in all there of these settings. At the same time, activities and programming in each are completely separate. By selecting these settings, the total possible sample size was maximized. The majority of youth participate in programming within the living unit, treatment groups, and school or vocation activities; thus few youth were excluded from the sample because these contexts applied to most, if not all, potential participants. 
These settings were also selected because the research literature points to clear benefits in gaining skills related to the living unit, treatment groups, and school or vocational activities while incarcerated. The school setting was selected because basic (e.g., reading, math) and higher education are consistently cited within the research literature as significantly reducing recidivism of incarcerated youth compared to those that do not participate in educational opportunities (Chappell, 2004; Fabelo, 2002; Gordon \& Waldon, 2003; Katsiyannis \& Archwamety, 1997; Katsiyannis, Ryan, Zhang, Spann, 2008). Not all OYA youth participate in educational programming because they are able to opt out of coursework once they receive a high school diploma or reach equivalency (i.e., GED). Collecting data from only those youth that participate in secondary or post-secondary education would have limited the sample size, so vocational activities were also included as part of this setting. Most youth that do not participate in formal education at OYA participate in vocational activities. There is also a broad base of support within the literature that connects vocational training while incarcerated to success in the community re-integration process (Bullis \& Yovanoff, 2002; Bullis \& Yovanoff, 2006; Dowden \& Andrews, 1999; Gordon \& Waldon, 2003). The educational and vocational setting is an important context within which to study psychological empowerment because skills learned within educational and vocational programming in correctional facilities are associated with job acquisition and job stability, educational attainment, and a crime-free future (Bullis \& Yovanoff, 2006; Chappell, 2004; Dowden \& Andrews, 1999).

Other correctional programming, including treatment and activities within the living unit, also help youth to engage in the community and avoid recidivating following 
release (Dowden \& Andrews, 1999; Todis et al., 2001). Treatment provided to youth with specific needs, such as substance abuse, anger or antisocial feelings, antisocial attitudes, and self-control, have all been found to reduce recidivism and predict community engagement (i.e., working, going to school, or both) following community reentry (Bullis \& Yovanoff, 2002; Dowden \& Andrews, 1999). Curricula within treatment and living unit activities build upon interpersonal, social, or other skills required in daily life. Such skills, including coping, problem-solving, and self-reflection, have been found to be characteristic of formerly incarcerated youth who have found some success in reintegrating into the community (Todis et al., 2001). Decision-making, which can be developed within treatment modules as well as within daily life activities, is also predictive of perceived post-detention success (Evans, Brown, \& Killian, 2002). In addition to skill-based activities within treatment and living units, pro-social activities within correctional settings are effective at reducing recidivism (Dowden \& Andrews, 1999). Many of these capacities, including self-control, anger awareness, coping, and problem-solving, are addressed within treatment groups and activities within living units; therefore, these settings were also included within this research.

\section{Conclusion}

Psychological empowerment has been presented as a useful tool for youth to develop during the time period in between childhood and adulthood. As discussed in Chapter III, empowerment programming can help youth to take on new roles and responsibilities, become active participants in their environment, develop pro-social and healthy relationships, and internalize pro-social values and norms (Chinman \& Linney; Mohajer \& Earnest, 2009). For incarcerated youth, skills, abilities, and knowledge 
developed through empowering processes may be particularly critical for the success of youth during the reintegration process. In fact, these skills may be essential to helping incarcerated youth make similar cognitive and behavioral changes as have been found in youth-based evaluation literature (Cargo et al., 2003). While psychological empowerment is theoretically beneficial to incarcerated youth, no research to support this notion has been conducted within this unique setting. Given that context is essential to the development of psychological empowerment, the importance of studying this construct within correctional facilities cannot be underscored more.

Empowerment can be developed in a variety of ways within any number of different programs at OYA. The living unit, treatment groups, and school or vocational activities were selected as settings within which psychological empowerment should be studied. These contexts were chosen because they maximized the total potential sample population, did not overlap, and have been found to relate to positive outcomes upon community re-entry. It is important to study psychological empowerment in different environments within OYA because it allows for the investigation of the extent to which youth experience the construct within different aspects of their lives within the correctional facility.

The justification for studying psychological empowerment within correctional facilities, specifically within OYA, has been presented within this chapter. In Chapter IV, an operationalization of psychological empowerment specific to the OYA context was put forth. The following chapters delve into the present study with the development of the research questions and hypotheses and the empirical findings related to the measure of psychological empowerment and its possible correlates. 


\section{CHAPTER VI}

\section{Development of Research Hypotheses}

The preceding sections have provided an overview of the literature on the juvenile justice system and psychological empowerment. Psychological empowerment was established as an important strength to cultivate within adolescent populations, and its possible benefits within youth correctional facilities were highlighted. Psychological empowerment was also placed within the study context, explaining the ways in which it may manifest within OYA. The following section will discuss the development of research hypotheses in the present study.

This research is the first of its kind to attempt to identify and measure psychological empowerment with a youth population in a correctional setting. The current study is the first to examine the factor structure of psychological empowerment and relationships between its primary components in three settings in this unique context. It is also the first study to investigate the short-term behavioral benefits of psychological empowerment for incarcerated youth. This study may contribute to the literature on constructs important to programming for incarcerated youth because no other empirical research has attempted to apply psychological empowerment to youth in correctional facilities. In addition to investigating psychological empowerment in a new context, its newly constructed measure was examined in three different settings in order to assess construct validity. These settings included the residential unit known as the living unit, treatment groups, and school and vocational activities. For detailed information about the study context, correctional and re-entry facilities for incarcerated youth run by OYA, see Chapter V. 
The Purpose of the Present Study

The purpose of the current study was to investigate psychological empowerment for youth residing within OYA correctional and re-entry facilities. More specifically, this study investigated the factor structure of psychological empowerment within three correctional facility settings: (1) residence, also known as the living unit; (2) treatment groups; and (3) school and vocational activities. The relationships between the primary components of psychological empowerment (i.e., intrapersonal, interactional, and behavioral) were explored in each of the three settings. After exploring the factor structure of psychological empowerment, the current study examined its effects in the three settings on behavioral success in five skill areas, known as domains, demonstrated by youth within OYA correctional and re-entry facilities.

\section{Research Hypotheses}

Relationships between dimensions of empowerment. As discussed in Chapter III (Empowerment Chapter), researchers have identified three primary components of psychological empowerment (i.e., intrapersonal, interactional, behavioral; Perkins \& Zimmerman, 1995; Zimmerman, 1995; Zimmerman \& Warschauski, 1998). No measure has been developed to assess the intrapersonal, interactional, and behavioral components of psychological empowerment in incarcerated youth populations. Without any model for measuring psychological empowerment in correctional facilities, a new measure was developed for use with incarcerated young men living within OYA correctional and reentry facilities (see Chapter IV for a description of the scale development process).

Because the intrapersonal, interactional, and behavioral components theoretically underlie psychological empowerment (Zimmerman, 1995; Zimmerman \& Warschauski, 
1998), it was hypothesized that the data would support a three-factor model and that the factors would be highly correlated. In each of the three settings within OYA correctional and re-entry facilities, this dissertation proposed that:

$H_{1 a}:$ A three-factor model will be supported by the data.

$H_{l b}$ : The Intrapersonal, Interactional, and Behavioral components of psychological empowerment will be highly correlated, indicating the presence of an underlying, higher-order factor.

Refer to Figure 3 for a visual representation of the three-factor model.

Psychological empowerment as a predictor of behavioral success. As suggested by proponents of the Good Lives Model (GLM) of sexual offender treatment (Marshall et al., 2005; Ward \& Mann, 2004) and Positive Youth Development for incarcerated youth (Bazemore \& Terry, 1997; Butts et al., 2005), aspects of psychological empowerment may help incarcerated youth lead healthy, pro-social lives. Evidence has suggested that psychological empowerment can increase engagement and participation, actualizing potential (i.e., esteem, confidence, competence, critical consciousness), control (e.g., taking responsibility, voicing opinions, taking action, decision making, confronting challenges, learning, and improving one's quality of life), and constructive change (e.g., youth development, success, and social integration; Cargo et al., 2003). These findings are indicative that psychological empowerment may help youth to create successful behavioral change. While behavioral change is a potential outcome of psychological empowerment, the extent to which youth cultivate this sense is related to the context in which it is developed. Psychological empowerment manifested in different settings within correctional and re-entry facilities should differentially predict behavioral success 
in various skill areas. In the present study, these skill areas or domains include: (1) education; (2) life and social skills; (3) offense-specific; (4) mental health; and (5) vocational. A set of three variables, which include age, total time incarcerated, and indeterminate versus determinate sentencing, served as controls in each of the hypotheses. These variables were included as controls because it was anticipated that each would explain additional variability in behavioral success. Indeterminate versus determinate sentencing was a distinction made between youth under OYA versus DOC jurisdiction. Youth within OYA are not sentenced with a specific length of time whereas youth under DOC jurisdiction must complete a minimum amount of time in the correctional facilities. It was hypothesized that:

Setting: School or Vocational Activities

$H_{2 a}$ : Controlling for age, total time incarcerated, and indeterminate versus determinate sentencing, psychological empowerment in school or vocational activities will positively predict behavioral success in the educational domain. $H_{2 b}$ : Controlling for age, total time incarcerated, and indeterminate versus determinate sentencing, psychological empowerment in school or vocational activities will positively predict behavioral success in the vocational domain.

Setting: Treatment Group(s)

$H_{2 c}$ : Controlling for age, total time incarcerated, and indeterminate versus determinate sentencing psychological empowerment in the youth's treatment group(s) will positively predict behavioral success in offense-specific domain. 
$H_{2 d}:$ Controlling for age, total time incarcerated, and indeterminate versus determinate sentencing, psychological empowerment in the youth's treatment group (s) will positively predict behavioral success in the mental health domain. $H_{2 e}$ : Controlling for age, total time incarcerated, and indeterminate versus determinate sentencing, psychological empowerment in the youth's treatment group(s) will positively predict behavioral success in the life/social skills domain.

Setting: The Living Unit

$H_{2 f}$ : Controlling for age, total time incarcerated, and indeterminate versus determinate sentencing, psychological empowerment in the living unit in which they reside will positively predict behavioral success in the life/social skills domain.

Refer to Figure 4 for the theoretical model underlying these hypotheses. 


\section{CHAPTER VII}

\section{Methods}

The purpose of the present study was twofold. First, this study examined the factor structure of psychological empowerment in three settings within OYA correctional and re-entry facilities. The second aim of this study was to examine the correlates of psychological empowerment in the same three settings. More specifically, this study investigated the relationship between behavioral success in five skill areas (i.e., OYA domains) and psychological empowerment. To accomplish the above goals, survey data was collected from young men within nine correctional and re-entry facilities as well as staff members' reports of youth behavioral success.

Few studies have investigated the development of strengths within the context of juvenile correctional and re-entry facilities. Within this setting, no research has been conducted on psychological empowerment. As a result of this gap in the research literature, there are no standardized or empirically supported quantitative tools to measure psychological empowerment in this unique context. A measure of psychological empowerment was, therefore, developed for use within OYA facilities.

\section{Institutional Review Board}

This dissertation project underwent review by two Institutions. First, the Oregon Youth Authority (OYA), the collaborating State agency, reviewed the project. Once approved by OYA, the Institutional Review Board at Portland State University reviewed and approved the present research.

\section{Study Context}


This study was developed principally as a dissertation research project. Although designed separate from other OYA research endeavors, the measure of psychological empowerment (the independent variable) was included in OYA's Annual Survey. OYA typically ask youth to complete one survey per year that assesses attitudes toward programming and climate with correctional and transition facilities. There was a natural connection between the psychological empowerment scales and the youth climate scales, so the researcher's newly constructed scale was incorporated into the annual survey. Due to the complimentary nature of the scales, data from the annual survey was incorporated into the dissertation.

This research was developed in collaboration with Dr. Shannon Myrick in the Research and Evaluation Unit at OYA. As discussed in Chapter IV, input was sought from the Youth Advisory Committee (YAC) at one of the nine correctional and re-entry facilities participating in this study. The YAC is a group of approximately 16 youth residing within one of OYA's correctional facilities. Their input was sought in order to gauge the language used as well as general content appropriateness of early versions of the measure. Their feedback was also utilized to improve validity, context specificity, and cultural appropriateness of the scales. For more information on OYA and the study context, refer to Chapter V.

\section{Participants}

Youth Participants. For detailed information about the sample, refer to Table 3. OYA houses up to 716 male youth in its nine correctional and re-entry facilities across the state. Data was collected from 550 male youth across the nine facilities, which was a response rate of approximately $77 \%$. Youth that did not participate in the study were 
either unavailable during data collection times due to prior engagements (e.g., work crew, visitation), declined participation, or were part of a unit where data collection was not possible due to behavioral issues. However, efforts to recruit participants that were unavailable during the first round of data collection were made by conducting a second round of data collection at each facility on a different day or time. Refer to Table 4 for sub-sample sizes per facility.

All youth within the nine facilities were young men between the approximate ages of 12 and 24. On average, youth were $18.43(S D=2.34)$ years old and had typically been incarcerated for $2.20(S D=1.94)$ years. The sample also included youth from various racial/ethnic backgrounds including African American (10.5\%), Anglo American (54.9\%), Asian (2.2\%), Latino (26.9\%), Native American (5.1\%), and "other" or unknown $(0.4 \%)$.

Participants were classified as either OYA (50.9\%) or DOC (49.1\%) commitment dispositions. Participants had been convicted of a range of crime types, including arson, criminal "other," person, property, public order, robbery, sexual offense, substance abuse/alcohol, and weapons. Nearly $38 \%$ of these crimes were characterized as a registerable offense (i.e., sexual offense).

Treatment Manager or Unit Coordinator Participants. The Treatment Manager or Unit Coordinator, who oversee individual OYA residential units, were recruited to complete a short survey on youth behavioral success based on competencies in five skill areas. Treatment Managers and Unit Coordinators oversee staff within each unit and are present at all meetings related to each youth. The Treatment Manager or Unit Coordinator was selected as the most appropriate OYA staff person to complete the 
surveys as $\mathrm{s} /$ he has access to the most information about each youth and has the most time and resources to be able to complete the survey (S. Myrick, personal communication, April 12, 2012). Additionally, both the Treatment Manager and Unit Coordinator have the opportunity to interact with youth in several contexts, including during treatment and in the unit. Only one of the two individuals, either the Treatment Manager or the Unit Coordinator, completed the survey for all the youth within a single residential unit. There are a total of 36 units within the nine OYA youth correctional and re-entry facilities. Either the Treatment Manager or the Unit Coordinator from each unit was recruited to participate; therefore, 36 Treatment Managers or Unit Coordinators were recruited. Data from 22 of the 36 units was returned. As a result, data on behavioral success from 22 Treatment Managers or Unit Coordinators was utilized (see Table 4 for a breakdown of data on youth competencies from staff respondents per facility).

Participant Recruitment

Youth Recruitment for Self-Report Data. All youth participants were recruited within the nine correctional or re-entry facilities that serve young men at OYA. Because the self-report measure of psychological empowerment was included in the OYA Annual Survey, research representatives from the Research and Evaluation Units at OYA and DOC were responsible for recruiting all participants. Youth participants were recruited from within their classes or vocational work crews. All youth present at the time of data collection were invited to participate. It was emphasized that their participation was not mandatory. All participants completed the OYA Annual Survey either online using the computer lab in the school associated with each facility or on paper. 
Treatment Manager or Unit Coordinator Recruitment for Data on Outcomes.

Thirty-six Treatment Managers or Unit Coordinators, one representing each unit, were invited to participate in data collection for the youth annual survey. Treatment Managers or Unit Coordinators were asked to complete the survey on the web for each youth within the unit during the youth annual survey. While it was encouraged that staff complete the survey on the same day that youth completed the OYA Annual Survey, several staff surveys were completed up to one month following data collection with the youth.

Procedure

The present study included two components. In the first part, self-report survey data on the scale of interest (i.e., psychological empowerment), which also served as the independent variable in later analyses, was collected from youth participants during the OYA annual survey. In the second part, data on the dependent variables were collected from the Treatment Manager or Unit Coordinator within each unit on the same day or within one month of self-report survey data collection with youth.

Self-report questionnaires were collected from 550 youth (i.e., all participants who were available and interested) in the nine correctional and re-entry facilities at OYA. As intended, the majority (66.4\%) took the survey online. Due to technical difficulties, a proportion of participants $(33.6 \%)$ were administered the paper-and-pencil version of the survey.

This research took place in conjunction with the OYA Annual Survey. In addition to the psychological empowerment, the self-report survey included other scales related to the correctional climate. Some of these scales were used to establish discriminant validity of the psychological empowerment sub-scales. These scales 
included measurements of locus of control, prison environment, and health care climate. The psychological empowerment scale consisted of three sub-scales measuring each of its primary components: (1) intrapersonal; (2) interactional; and (3) behavioral. Refer to Appendix A for a complete version of the OYA Annual Survey. The self-report survey took youth between 20 and 45 minutes to complete. Accommodations were made for those youth that had difficulty reading. For these youth, the survey was read aloud. Following completion of the survey, youth received a small token of appreciation (i.e., a granola bar), which was provided to the youth by the Research and Evaluation Unit at OYA. Basic demographic information (e.g., age, ethnicity, total time incarceration) was collected from the Juvenile Justice Information System (JJIS). Data from the OYA Annual Survey and JJIS were matched and entered into a single database.

Data on the dependent variables (i.e., behavioral success in five domains) was collected either on the same day or within 30 days of data collection of the independent variables. Data on behavioral success was collected directly from the Treatment Manager or Unit Coordinator within each OYA unit. Refer to Appendix B for a copy of the staff survey that was used to measure behavioral success in the five specified OYA domains. Data from staff were collected using a web-based surveyor (i.e., Survey Monkey). Finally, data from each Treatment Manager or Unit Coordinator were matched to self-report survey data provided by youth during the OYA Annual Survey and demographic data from JJIS.

Design

A cross-sectional, non-experimental design was utilized. Specifically, quantitative survey data was collected from young men within OYA correctional and re- 
entry facilities. Quantitative data on the independent (i.e., self-report survey data on psychological empowerment) and dependent (i.e., OYA staff report data on behavioral success) variables, in the form of self-reported survey data from youth and Treatment Manager- or Unit Coordinator-reported behavioral data, was measured at one time point. Quantitative Measures

Demographic variables. Demographic information was collected from JJIS using each participant's OYA identification number. Items collected from JJIS included age, ethnicity, previous convictions, total time incarcerated, and commitment disposition (i.e., OYA or DOC).

Psychological Empowerment. As outlined in Chapter IV, no measure of psychological empowerment assessing all of its three components within a youth or young adult, incarcerated population exists. For this reason, three self-report survey subscales measuring the components of psychological empowerment in three settings within OYA (i.e., treatment groups, school or vocational activities, and their residence or living unit) were created specifically for use in OYA correctional and re-entry facilities. For an in-depth discussion of the development of the measure of psychological empowerment scale constructed for use within the OYA system, refer to Chapter IV.

Eighteen items assessing psychological empowerment in one of three settings were measured on a five-point Likert scale from "Strongly Disagree" (1) to "Strongly Agree" (5). Half of the items (9 total items) were reverse or negatively worded and were subsequently reversed coded. Responses to each of the items were made in regards to three different settings within OYA. These settings included youths' residence or living unit, treatment groups, and school and vocational activities in which they were involved. 
Refer to Table 1 for the psychological empowerment scale items within each setting and their abbreviations. Appendix A contains a copy of the survey as administered to youth.

Each component of psychological empowerment was composed of six total items, one positively and one negatively worded item representing each of the components' three domains. Within each setting (i.e., treatment group, school or vocational activities, living unit), it was intended that each set of 6 items were to be averaged to create a single composite score for each component of psychological empowerment within the setting. Furthermore, there was a potential total for 9 composite scores representing psychological empowerment, one for each of the three components (i.e., Intrapersonal, Interactional, Behavioral) in each of the three settings. Higher scores on each of the composite variables indicated higher psychological empowerment within each of its components.

Results from confirmatory factor analyses did not justify the use of the planned composite scores. Instead of the planned composites, an average composite score for items that were supported under respecified factor structures was calculated and utilized within inferential analyses (see results below).

Behavioral success. OYA staff ratings on youth competencies within five OYA domains $^{3}$ were measured in order to assess behavioral success. OYA has identified several domains or skill areas, including "education," "mental health," "offense specific," and "substance abuse," that youth develop during their time in the facilities. Over 200

\footnotetext{
${ }^{3}$ Note that behavioral success was measured in five different competency areas, called domains. Three of these domains overlapped with the settings in which psychological empowerment was assessed (i.e., treatment, school, and vocation). In addition to the treatment groups, school, and vocational training, the competencies also included skill areas that were not specific to the three settings (i.e., mental health, offense-specific, life/social skills). Thus, in accordance with OYA terminology, "domain" is used to describe the five competencies.
} 
competencies, which are related to specific goals for each youth, are associated with the domains identified by OYA. OYA selects competencies for each youth based on his RNA and treatment plan; thus competencies assessed vary between youth. For the purposes of this study, five domains were selected as skill areas to assess. These five domains included: (1) education; (2) life/social skills; (3) mental health; (4) offense specific; and (5) vocational. Selection of domains was based on skill areas most related to the research and that were anticipated to pertain to the majority of participants in the study. Within each domain, several competencies were chosen from a list of related competencies provided by OYA. The inclusion of competencies was based on relevance to the study, relevance to the broad population (i.e., competencies likely to apply to most youth), and the competencies' abilities to assess the domain broadly. Four competencies were chosen to measure education, three were chosen to measure family, five were chosen to measure life/social skills, five were chosen to measure mental health, five were chosen to measure offense specific, and four were chosen to measure vocation.

The Treatment Manager or Unit Coordinator within each unit rated each youth on the competencies associated with each of the five domains. Ratings were made based on conversations with teachers, the work crew manager or other supervisors, and other OYA staff. Each response stem was on a 5-point Likert scale, where "1" indicated "Almost Never: The youth shows little or no ability to demonstrate the competency," and "5" indicated "Almost Always: The youth is able to demonstrate the competency at least 90$100 \%$ of the time." This rating scale was familiar to OYA staff, which rate competencies on this metric every 90 days in JJIS. Although staff rate competencies periodically, Treatment Mangers or Unit Coordinators were asked to rate selected competencies in a 
separate survey, apart from JJIS. They were asked to do so because competencies within JJIS are not universal across all youth. Thus, this was the first attempt at rating competencies determined to apply to most, if not all, youth at OYA. Refer to Appendix B for a complete copy of the OYA staff survey.

In order to create one composite score for behavioral success within each of the five domains, ratings of the competencies corresponding to each of the domains were averaged. Higher composite scores for each domain represented higher behavioral success whereas lower scores represented lower behavioral success. In order for the composite score to be calculated, an $80 \%$ response rate was required for items within each domain. In the few cases where $80 \%$ item completion was not achieved, no composite score was calculated.

Prison Environment. In addition to the PE scale developed for the present study, the OYA Annual Survey also included a measure of climate of the correctional setting. The Hybrid Prison Environment Scale (HPES) was developed by OYA researchers based on two previous assessments of prison climate (van der Helm, Stams, van der Laan, 2011; Wright, 1985). The Prison Environment Inventory (PEI; Wright, 1985) consisted of 80 items that assessed eight domains of the prison setting: (1) Privacy; (2) Safety; (3) Structure; (4) Support; (5) Emotional Feedback; (6) Social Stimulation; (7) Activity; and (8) Freedom. The scale and each of its eight sub-scales was found, in general, to have acceptable internal reliabilities for early stages of research. Wright (1985) reported that reliabilities for only two of the eight sub-scales within the PEI did not meet the standards for early stages of research. Items within the HPES were also based on the 63-item Prison Group Climate Instrument developed by van der Helm and colleagues (2011) to 
measure four domains of the prison environment: (1) Repression; (2) Support; (3) Growth; and (4) Group Atmosphere. Their four-factor model was supported, and subscales were reported to have good reliability (Cronbach's alpha of .76 and above for all sub-scales). Although the PGCI was constructed for use with incarcerated juveniles and adults, it was originally developed in the Netherlands. Translations of the 63-items from Dutch to English were questioned by the researchers at OYA; therefore, the PGCI and PEI were combined to create the HPES. Only select items from the PEI and PGCI were adapted to fit the American youth correctional setting. These items (see Appendix C) were hypothesized to measure eight correctional domains: (1) Activity; (2) Emotional feedback; (3) Freedom; (4) Growth; (5) Privacy; (6) Social stimulation; (7) Structure; and (8) Support. They were measured on a 5-point Likert scale from 1 (“Strongly Disagree”) to 5 (“Strongly Agree”), where higher scores were indicative of more positive attitudes about the environment within each domain. In the present study, the HPES had excellent internal reliability (Cronbach's $\alpha=.96$; see Table 5). A total composite score was calculated for the HPES.

Locus of Control. The OYA Annual Survey also included a 16-item scale measuring locus of control (LOC). The scale was based on Craig, Franklin, and Andrew's (1984) 17-item measure of locus of control of behavior (LCB). The LCB was scored on a 6-point Likert scale from 0 (“Strongly Disagree”) to 5 (“Strongly Agree”), where higher scores indicated externality and lower scores indicated internality. Craig and colleague's (1984) scale was found to be invariant across age, sex, and social desirability and to have good test-retest reliability (.90). Evidence also supported construct validity of the LCB. Researchers at OYA determined that some of the items in 
the LCB did not translate to the correctional setting well. In order to tailor the scale to the context, modifications were made. Typically, revisions were made in order to simplify the language of each item. Additionally, one item, which was thought to be redundant, was eliminated. OYA's version, hereafter referred to as LOC, was scored on a 5-point Likert scale from 1 ("Strongly Disagree") to 5 ("Strongly Agree"). Scoring reflected that within the original scale, where higher scores on the sum of all items was associated with externality. The LOC scale had good internal consistency (Cronbach's $\alpha$ $=.82$; see Table 5). Refer to Appendix D for the LOC items used within the OYA Annual Survey.

Health Care Climate. The degree of autonomy in treatment was also measured within the OYA Annual Survey. This 15-item scale was based on the Health Care Climate Questionnaire (HCCQ) developed by Williams and colleagues (1996). The HCCQ was scored on a 5-point Likert scale from 1 ("Not at all true") to 5 ("Very true") as was found to have high internal reliability (.95). OYA made minor revisions to the language of the items in order to simplify the measure and in order to fit the items to the correctional treatment context. The final version of the HCCQ (see Appendix E) was measured on a 5-point Likert scale from 1 ("Strongly Disagree") to 5 ("Strongly Agree"), where higher scores on the aggregate composite were associated with feelings of autonomy. As indicated in Table 5, the HCCQ had excellent reliability (Cronbach's $\alpha=$ $.97)$. 


\section{CHAPTER VIII}

\section{Results}

All statistical analyses examining descriptive statistics as well as assessing the hypotheses were conducted in SPSS 20.0 and Mplus 7.0. A series of confirmatory factor analyses (CFA) were conducted to address the first set of hypotheses (1a and 1b). Hierarchical linear modeling (HLM) was used to assess the second set of hypotheses (2a2f). Before these analyses were conducted, the data were explored descriptively.

\section{Data Screening}

Prior to inferential data analyses for hypothesis testing, all data were screened for missing values, patterned responses, outliers, multicollinearity, and assumptions of the General Linear Model. First, frequency distributions and range statistics were examined to determine if any data outside the plausible scale scoring existed. No cases with data points outside of the plausible range of scores were identified.

Second, response patterns were investigated by examining composite scores for PE scales on original items (i.e., prior to reverse coding). Six cases were identified as having patterned data on two or more psychological empowerment scales or on one PE scale and at least one other scale within the survey. Sixty-seven percent of these cases consistently reported "5" or "Strongly Agree" across all items in the scales. These six cases were dropped and were not included in further analyses. Additionally, two cases were dropped from the dataset because these participants were flagged during data collection as having paid little attention to the survey.

Third, outliers were assessed. Prior to conducting CFAs, multivariate outliers were examined using the Mahalanobis Distance statistic for each PE sub-scale. Seven 
cases were identified as problematic as their responses indicated a tendency to dichotomize the response scale (i.e., responses were "1" or "Strongly Disagree" and "5" or "Strongly Agree"). These seven cases were dropped and were not included in further analyses.

Next, multicollinearity was assessed using the Tolerance statistic, where Tolerance (1-SMC) below .10 was considered suggestive of issues of multicollinearity (Tabachnick \& Fidell, 2007). Prior to conducting CFAs, no multicollinearity between PE items was found.

Following results from the CFAs, outliers on the composite scores for factors that were supported (i.e., Intrapersonal PE in three settings) were assessed through an inspection of scatterplots, boxplots, and frequency distributions. Scores that were three standard deviations beyond the mean were characterized as univariate outliers (Howell, 2002). One to two univariate outliers were identified on composite scores of supported factors. All univariate outliers were low on Intrapersonal PE. Univariate outlying scores were changed to the next lowest, non-outlying score minus one unit.

Additionally, univariate outliers were examined within the other sub-scales and scales that were included in the OYA Annual Survey (i.e., Hybrid Prison Environment Scale, Locus of Control Scale, Health Care Climate Questionnaire) as well as the scales that the Unit Coordinators completed for each youth (i.e., DVs). Outlying scores that were three or more standard deviations above or below the mean were changed to the next highest or lowest score plus or minus one unit, respectively.

In order to prepare the data for analyses to examine the relationship between components of PE and behavioral success, multivariate outliers on Intrapersonal PE (i.e., 
the empirically supported factor structures), the control variables (i.e., age, total time incarcerated, and commitment disposition), and the dependent variables (e.g., behavioral success in the educational, life skills, mental health, offense-specific, and vocational domains) were assessed. Two multivariate outliers were identified as significant using the Mahalanobis Distance statistic and its associated inferential test. The two multivariate outliers were determined to be part of the overall population of incarcerated youth; therefore, their scores were retained in the sample.

Multicollinearity was also assessed for control and predictor variables used in HLM analyses on behavioral success. Although there were a few issues of multicollinearity when Tolerance and VIF were examined for each set of predictors within each living unit (i.e., grouping variable), it was evident that multicollinearity was not a problem in analyses across units. All Tolerance statistics were greater than .10 and all VIF statistics were less than 10.0, when multicollinearity was assessed across living units. Although there were no issues with multicollinearity of predictors across units, each of the continuous predictors, including the covariates, was grand mean centered for interpretational purposes.

Finally, following analyses of the factor structure in each of the three settings, the assumptions associated with the General Linear Model were examined. Specifically, descriptive statistics and exploratory regression analyses were conducted in order to examine skewness, kurtosis, histograms of residuals, normal P-P plots, and scatterplots of residuals. First, three dependent variables were found to be skewed and/or kurtotic within specific facilities. Exploratory regression analyses on each dependent variable in each unit (i.e., grouping variable) indicated that not all the assumptions were satisfied in 
every living unit. However, all exploratory regression analyses were conducted with 24 or fewer cases, with the smallest regression conducted with only six cases. Furthermore, it is not surprising that normality, linearity, and homoscedasticity did not hold within each unit because of small sub-sample sizes. For this reason, the assumptions were also checked through exploratory regression analyses on behavioral success across living units. Histograms as well as skewness and kurtosis statistics indicated that the distributions of each of the dependent variables were normal. Results of normal P-P plots for behavioral success in each domain (i.e., education, life skills, mental health, offensespecific, vocation) reflected this finding. The assumptions of linearity and homoscedasticity were also determined to be satisfied through inspections of residual histograms and scatterplots. Note that, although original values on the dependent variables were utilized in subsequent analyses, analyses were also conducted on transformed dependent variables (reflected square root of the dependent variable) in order to compare findings. The results of analyses using transformed dependent variables were substantively the same.

Confirmatory Factor Analysis on the Correlated Three-factor Psychological

\section{Empowerment Model.}

To address the research hypotheses stated in Chapter VI, several analyses were conducted. These analyses included a series of confirmatory factor analyses (CFAs) and hierarchical linear modeling (HLM). The following section will detail the analyses conducted to address each of the research questions related to the factor structure of psychological empowerment.

$H_{1 a}:$ A three-factor model will be supported by the data. 
$H_{1 b}:$ The Intrapersonal, Interactional, and Behavioral components of

psychological empowerment will be highly correlated, indicating the presence of an underlying, higher-order factor.

Prior to conducting a confirmatory factor analysis (CFA) for the three-factor model, correlation matrices for each setting were examined in order to gauge the degree to which items were associated with one another and with each of the composite scores representing the hypothesized latent factors (i.e., average score of six indicators). Refer to Tables 6,7 , and 8 for correlation matrices for each component of psychological empowerment. Note that these correlations were calculated using SPSS, which utilized listwise deletion for missing data (between 5 and 14 missing cases per bivariate correlation). Based on the correlation matrices, it was anticipated that there would be difficulties with model fit in all three settings. Specifically, there were inter-item correlations that were near zero as well as negative inter-item correlations.

CFAs were conducted using Mplus 7.0 software. Due to missing data, which was assumed to be missing at random, all CFAs were conducted using the Full Information Maximum Likelihood (FIML) technique. Models were determined to fit the data well using a combination of indicators, including when: (1) the chi-square goodness of fit statistic was non-significant; (2) the standardized residual covariance matrix demonstrated no large discrepancies between the sample implied and model covariance matrices above $|2.0|$; (3) the Comparative Fit Index (CFI) was greater than .95; and (4) the Root Mean Square Residual (RMSEA) was less than .10 (Hu \& Bentler, 1999; Kline, 2011; McDonald, 1999). 
As discussed later, exploratory factor analyses (EFAs) were also conducted to aid in model specification. All EFAs were conducted using the Direct Oblimin rotation, when more than one factor was extracted. The final number of factors for each model was selected through a combination of Kaiser's criteria and Cattell's scree plot. Additionally, it was required that all items load on to each factor saliently (i.e., factor loading of .30 or higher).

In order to address Hypotheses $1 \mathrm{a}$ and $1 \mathrm{~b}$, the factor structure of the scale measuring psychological empowerment and its three components in three different settings was examined (see Figure 3). The scale assessing psychological empowerment in the proposed study has never been used to measure the construct of interest. For this reason, three CFAs were conducted, one for each of the three settings. Theoretical literature supports the notion that psychological empowerment underlies the three components (i.e., intrapersonal, interactional, and behavioral) measured in the current study (Perkins \& Zimmerman, 1995; Zimmerman \& Warschauski, 1998). Aligned with the research literature, the fit of a three-factor model, which was over-identified (i.e., degrees of freedom $[\mathrm{DF}]=132)$, was examined.

As specified in Figure 3, six pure indicators (i.e., items) were explained by the Intrapersonal component, six pure indicators were explained by the Interactional component, and six pure indicators were explained by the Behavioral component of psychological empowerment. A unit loading identification constraint (1.0) was placed on the paths from the measurement error terms to their indicators for scaling purposes. Similarly, the unstandardized factor loadings of three items were constrained to 1.0, also for scaling purposes. Scaling constants were placed on the paths from SE2 to 
Intrapersonal psychological empowerment, from AR1 to Interactional psychological empowerment, and from A1 to Behavioral psychological empowerment. As suggested by Kline (2011), the selection of placement of the unit loading identification constraint is arbitrary when each indicator is equally reliable. Additionally, each of the three latent factors representing the three components of psychological empowerment were allowed to covary with one another. Finally, due to missing data, the Full Information Maximum Likelihood (FIML) parameter estimation technique was utilized to estimate model parameters (Kline, 2011; McDonald, 1999). This three-factor model was assessed in each of the three settings (i.e., living unit, treatment, school or vocation).

The three-factor structure was not supported in any of the three settings. Correlations between the three latent factors were high (from $r=.79$ to over 1.0 in the three settings). In fact, the latent factors (i.e., Intrapersonal, Interactional, and Behavioral PE) were so highly correlated that the models were non-positive definite, creating implausible correlation estimates (i.e., greater than 1.0) in the living unit and treatment settings. As a result of non-positive definiteness, all parameter estimates produced were invalid. An alternative model specifying a higher-order factor (i.e., PE) within each setting was investigated by conducting hierarchical CFAs in order to examine the error variances of these three first-order latent factors. In each of the three settings, most of the disturbance variances of the three latent factors were less than $|.1|$, suggesting that the first-order factors measured exactly the same construct. Refer to Table 9 for latent factor correlations and for disturbance variances of latent factors in each setting.

As a result of non-positive definiteness of each of the three models and nil to medium inter-item correlations within first-order factors (see Tables 6, 7, and 8), 
exploratory analyses were conducted in order to investigate the factor structure of each of the first-order factors. The PE scale within the living unit setting was presented first within the OYA Annual Survey; therefore, further analyses were conducted on the PE sub-scales in the living unit first. Subsequent analyses used results from the living unit setting as a basis for model respecification. The following discussion of results will detail the factor structure findings for each component of PE. Results for each setting under each PE component will be discussed in order of appearance in the survey, beginning with the living unit, then treatment groups, and finally school or vocational activities.

\section{Confirmatory Analyses on the Factor Structure of Intrapersonal PE}

Intrapersonal PE in the Living Unit. In order to assess each of the three firstorder factor structures in the living unit setting, split-half exploratory factor analyses (EFAs) followed by CFAs were conducted. First, an EFA on Intrapersonal PE in the living unit setting was conducted. All six items were entered into the EFA, which extracted two factors with eigenvalues 2.26 and 1.05 using Kaiser's criterion (i.e., eigenvalues greater than 1.0). Kaiser's criterion is suggested to over-extract factors (Tabachnick \& Fidell, 2007); therefore, Cattell's scree plot was examined to compare the number of factors extracted. The scree plot indicated that only one factor was present. In accordance with theory, it was determined that there was sufficient evidence for unidimensionality based on the scree plot.

The EFA was rerun, specifying the extraction of a single factor. The total variance in the items explained by the single factor was $25.95 \%$. The majority ( 5 out of 6) of items loaded saliently on to the factor; however, one item, the reverse coded item 
for perceived control or "There is little I can do to change the things that are important to me," had a non-salient factor loading of .26. Therefore, this item was eliminated from the model, and the EFA was again rerun.

The third EFA produced the final model, which consisted of 5 of the 6 original items (i.e., SE1, SE2, MC1, MC2, and PC1). The single factor explained $30 \%$ of the variability in the five items. All items loaded saliently on to the Intrapersonal factor (see Table 10). Factor loadings ranged from .40 (MC2) to .66 (SE2), and the residual correlation matrix indicated only few discrepancies between the sample and reproduced correlation matrices. Only one residual correlation (-.11), between SE1 and MC1, was greater than $|.10|$. Furthermore, this single-factor model with five indicators was determined to be satisfactory.

Next, a split-half CFA was conducted in order to confirm the results of the splithalf EFA on Intrapersonal PE. The model was specified as having five items explained by the Intrapersonal PE factor and no error variances were specified as correlated (see Figure 5). This model reflected the original Intrapersonal PE factor from the initial CFA with the exception that PC2 was eliminated from the model. This model was overidentified $(d f=5)$ with the path between Intrapersonal PE and SE2 constrained to 1.0. FIML was used to estimate the model parameters.

In order to evaluate the model fit, the chi-square goodness of fit statistic, the standardized residual covariance matrix, and fit indices were examined. The model fit indices suggested borderline adequate fit to the data. The chi-square goodness of fit test was significant, $\chi^{2}(5)=19.99, p=.001$, rejecting the null hypothesis of exact model fit. It is possible that the significant chi-square value was due to its sensitivity to sample sizes 
$(N=273$; Wegener \& Fabrigar, 2000); therefore, both relative and parsimony-adjusted fit indices were examined. These fit indices suggested borderline adequate model fit, where the CFI was .92 and the Root Mean Square Error of Approximation (RMSEA) was .11. To better determine if the model adequately fit the data, the standardized residual covariance matrix was examined. Although the fit indices suggested borderline adequate fit, there were several serious discrepancies between the sample and model implied covariance matrices, which exceeded the cutoff of $|2.0|$.

In order to improve the model fit by respecifying the model, estimated error variances and modification indices were assessed. First, the errors between items SE2 and $\mathrm{MC} 1$, which were both positively worded items, were allowed to covary. After doing so, the standardized factor loading for item MC2 was reduced (.27), and the estimated residual or measurement error variance of MC2 (1.05) became much higher than that of any other indicator (ranging from .11 to .77). The estimated sample correlations between MC2 and all other items were generally the lowest in the matrix. To further explore this item's impact on the sub-scale, the average inter-item correlation was examined with and without item MC2 through Cronbach's alpha if item MC2 was deleted. The average inter-item correlation was higher without item MC2 $(\alpha=.66$ without item MC2 compared to $\alpha=.65$ with item MC2); therefore, item MC2 was dropped. This decision was also supported by the fact that the reverse coded items did not load as highly onto the factor as the other, positively worded items and because item PC2, which was also reverse coded, was dropped in the EFA process.

The model was then rerun, and a final modification to the model was made based on results. In the final model, the residuals between items SE1 and PC1 were allowed to 
covary. These items were allowed to covary because they were originally worded in opposite directions (i.e., item SE1 was negatively worded, and item PC1 was positively worded); thus, a negative relationship was expected between estimated residuals.

The final model fit the data well (see Table 11 for standardized factor loadings and standardized estimated error variances and Table 12 for fit indices). The chi-square goodness of fit statistic was not significant, $\chi^{2}(1)=1.15, p=.28$, implying exact model fit. Additional fit indices $(\mathrm{CFI}=.99$ and $\mathrm{RMSEA}=.02)$ reflected chi-square findings. As illustrated in Table 13, the standardized residual covariance matrix also suggested good model fit, with discrepancies between sample and model implied covariance matrices less than $|2.0|$. Standardized factor loadings ranged from .48 to .74. As noted earlier, the highest standardized factor loadings were associated with items that were positively worded in the survey. Positively worded items were also estimated to have the least amount of residual or measurement error variance. Finally, the measurement errors for items SE1 and PC1 were negatively correlated (-.35). Refer to Figure 6 for the final model of Intrapersonal PE.

Intrapersonal PE in Treatment. The empirically supported CFA on Intrapersonal $\mathrm{PE}$ in the living unit consisted of four items (SE1, SE2, MC1, and PC1), and the residual terms between items SE1 and PC1 were allowed to covary. In order to test the model fit for Intrapersonal PE in the treatment setting, a CFA on this model (see Figure 6) was conducted for responses related to the treatment setting.

Results from the CFA demonstrated that the single factor model for Intrapersonal PE was supported in the treatment setting. The chi-square goodness of fit statistic was non-significant, $\chi^{2}(1)=.03, p=.87$, indicating exact model fit. Fit indices were also 
perfect (i.e., $\mathrm{CFI}=1.0$ and RMSEA $=.00$; see Table 12). An inspection of the standardized residual covariance matrix suggested only minor discrepancies between the sample and model implied covariance matrices (see Table 13). As depicted in Table 11, standardized factor loadings were similar to those in the living unit setting with the exception of item PC1, which was much lower. Again, measurement error terms for items SE1 and PC1 were negatively correlated (-.10).

Intrapersonal PE in School or Vocation. The model for Intrapersonal PE (see Figure 6) was also tested within the school or vocational setting. Similar to results from the other two settings, the single factor model for Intrapersonal PE was supported in the school or vocational setting. The chi-square goodness of fit statistic was non-significant, $\chi^{2}(1)=.90, p=.34$, indicating that there was no significant difference between the sample and model implied covariance matrices. Results of the fit indices were also indicative of model fit (see Table 12). After rounding, the CFI and RMSEA were perfect, 1.0 and 0 , respectively. The standardized residual covariance matrix, which can be seen in Table 13, confirmed that the majority of discrepancies between the sample and model implied covariance matrices were small. However, note that the discrepancy for items $\mathrm{MC} 1$ and PC1 was more than twice that of the common cutoff $(|2.0|)$. Additionally, the correlation between the measurement errors for items SE1 and PC1 was close to zero (.01), suggesting that this correlation might have been unnecessary. Finally, the magnitude of factor loadings was similar across items, with the exception of item SE2, which was higher than any other indicator (see Table 11). Nevertheless, the model for Intrapersonal PE in school or vocation was determined to fit the data well; however, caution must be used when interpreting this factor model and when utilizing its 
composite score in further analyses due to potential model misfit reflected in the discrepancy found in the standardized residual covariance matrix between items MC1 and PC1.

Measurement Invariance of Intrapersonal PE. A large percentage of youth that completed the OYA Annual Survey on the paper-and-pencil survey format (33.6\%). As a result, the variability in the measurement of Intrapersonal PE between paper-and-pencil and online survey formats was explored in each setting. Measurement invariance was first examined by comparing the mean scores of Intrapersonal PE between survey administration formats. Descriptively, participants that completed the annual survey online reported higher Intrapersonal PE scores in all three settings. As illustrated in Table 15, this difference was significant in the treatment and school or vocational settings, $t(263)=-2.10, p=.04$ and $t(266)=-2.14, p=.03$, respectively.

Potential differences were further explored through CFAs. First, configural invariance was examined in the living unit setting. The configural invariance model (i.e., Step 1) allowed the single-factor structure that was supported through the split-half CFA to be compared between data that was collected via paper-and-pencil format and via online format. Step 1 allowed factor loadings and estimated error variances to vary between survey administration formats; however, the same unit loading identification, on the path between Intrapersonal PE and item SE2, was used. Results from this first step indicated that the measurement of Intrapersonal PE was not consistent across paper-andpencil and online survey administrations. The factor structure on the paper-and-pencil survey administration format was found to be non-positive definite, where the estimated error variance of item $\mathrm{MC1}$ was negative (-.03). In contrast, the estimated error variance 
of item $\mathrm{MC} 1$ in the online format (.59) was more representative of other error variances in both survey administration formats. Due to non-positive definiteness of the factor structure in the paper-and-pencil format, measurement invariance was not explored further in the living unit setting.

Configural variance was also found in the school or vocational setting. The single-factor structure for the online survey administration was non-positive definite. In contrast to results in the living unit setting, however, item SE2 was responsible for the non-positive definiteness, with an estimated error variance of -.11.

Finally, measurement invariance was examined in the treatment setting. Through a series of gradually more stringent analyses, it was determined that the two survey administrations were equivalent in estimated residual variances in the treatment setting (see Table 16). First, configural invariance was examined, and the models fit the data well. Next, the factor loadings were constrained to be equal across administrations, and this model fit the data well. Additionally, the chi-square difference test was nonsignificant, $\chi^{2}(3)=1.78, p=.62$, suggesting that the model with construct metric invariance did not fit worse than the model with configural invariance. The third model restricted the intercepts to be equivalent across survey administrations, and the model again fit the data well (difference in $\chi^{2}(4)=6.39, p=.17$ ). Finally, equivalence in estimated residual variances was examined. The model fit the data well, and the chisquare difference test was non-significant, $\chi^{2}(4)=6.72, p=.15$, demonstrating no significant difference between the scalar and residual invariance models. Reliability Assessment of Intrapersonal PE 
Internal consistency (i.e., internal reliability) was assessed for Intrapersonal PE by examining Cronbach's alpha. Scales with Cronbach's alpha equal to or greater than .72 were considered to have acceptable reliability, and scales with Cronbach's alpha of .80 or greater were considered to have good reliability (John \& Benet-Martinez, 2000). Internal consistency for the Intrapersonal PE sub-scale was calculated for each of the three settings. Cronbach's alpha was relatively low, ranging from .60 in the treatment setting to .66 in the living unit. These calculations did not meet the standard criteria for acceptable reliability for Intrapersonal PE. Refer to Table 5 for Intrapersonal PE subscale reliabilities within each setting.

\section{Validity of Intrapersonal PE}

Comparisons of Intrapersonal PE Model Fit Across Settings. After examining model fit for Intrapersonal PE, results were compared across the three settings. The single-factor for Intrapersonal PE, which was composed of four indicators and one covariance between two measurement error terms (items SE1 and PC1), was found to fit the data well in each of the three settings. In fact, the chi-square goodness of fit statistic was non-significant in all three settings, and differences in the chi-square across settings were minimal. The CFI and RMSEA suggested perfect fit (i.e., 1.0 and .00, respectively) in the treatment and school or vocational settings, and near perfect fit in the living unit setting (.99 and .02, respectively). Standardized factor loadings for each indicator were, however, slightly different across settings. Most notably, the item with the highest standardized factor loading in the living unit setting was item PC1 (i.e., perceived control domain) whereas the item with the highest standardized factor loading in the treatment and school or vocational settings was item SE2 (i.e., self-efficacy domain). Despite this 
discrepancy, the standardized factor loading for item SE2 was also high within the living unit setting. The most concerning difference between models in the three settings was that the standardized residual covariance matrix in the school or vocational setting indicated a serious discrepancy between items MC1 and PC1. A model that allows for these items to covary instead of items SE2 and PC1 might fit the data better in the school or vocational setting. Overall, however, there were few descriptively noteworthy differences between the Intrapersonal PE model fit across the three settings. Therefore, it was determined that the operationalization of Intrapersonal PE was relatively consistent across these three settings. Notice that correlations between these factors were large, with Pearson's product moment correlation ranging from .69 to .74 (see Table 14).

Discriminant Validity of Intrapersonal PE. Discriminant validity of Intrapersonal PE was examined through correlation matrices (see Tables 17 to 22). The relationship between Intrapersonal PE in each of the three settings and the Hybrid Prison Environment Scale (HPES), Locus of Control (LOC), and the Health Care Climate Questionnaire (HCCQ) were assessed. Evidence supported the notion that Intrapersonal PE was a distinct construct, with correlations ranging from .20 to -.53 . Notably, the highest correlations were between LOC and Intrapersonal PE, which was consistent across all three correctional settings $(r=-.48$ in the living unit, $r=-.52$ in treatment, and $r=-.53$ in school or vocation). The correlations between LOC and Intrapersonal PE in the three settings were negative. This was due to the scoring of LOC, where higher scores were associated with externality as opposed to internality. The sub-scales that correlated lowest with Intrapersonal PE, across all three settings, were the Freedom and Privacy sub-scales within the HPES, ranging from Pearson's product moment correlation 
of .19 to .24 and ranging from .26 to .28 , respectively. Finally, it should be noted that Intrapersonal PE correlated with HCCQ differently across settings. Specifically, the correlation between Intrapersonal PE and HCCQ was highest within the treatment setting $(r=.39)$. This result was to be expected as HCCQ items referred most to the correctional treatment setting. Confirmatory Analyses on the Factor Structure of Interactional PE

Interactional PE in the Living Unit. In order to examine the factor structure of the six items within the Interactional PE factor, a split-half EFA was conducted. All six items were entered into the model, and, according to Kaiser's criterion, three factors were extracted. Although three factors were extracted, there was not much variability in the initial eigenvalues, suggesting that Kaiser's criterion, which extracted eigenvalues greater than 1.0, was somewhat arbitrary. An inspection of Cattell's scree plot reflected this finding and did not aid in model specification as there was no natural break in eigenvalues per factor. The pattern matrix indicated that only one item saliently loaded on to Factor 1, two items loaded on to Factor 2 saliently, and only one item loaded on to Factor 3 saliently (see Table 10). There was one complex item (AR1), and two of the six items (CA2 and PS2) did not load saliently on to any factor. For further model clarification, inter-item correlations were examined (see Table 7). Inter-item correlations were very low, with the majority $(60 \%)$ of correlations less than $|.10|$, and the highest inter-item correlations between items AR1 and PS1 $(r=.32)$. While most of these correlations were close to zero, many $(47.70 \%)$ were also negative. Furthermore, it was determined that these items did not "hang well together;" thus, dimensionality was not 
defined. As a result, no additional EFAs were warranted and CFAs were not conducted on Interactional PE in any of the three OYA settings.

\section{Confirmatory Analyses on the Factor Structure of Behavioral PE}

Behavioral PE in the Living Unit. A split-half EFA/CFA was conducted on the six items that were theoretically explained by Behavioral PE using the Direct Oblimin rotation to fit the pattern loadings. The EFA extracted two factors with eigenvalues of 2.20 and 1.11. However, Cattell's scree plot suggested unidimensionality among the six items. Based on the results of the scree plot, it was determined that there was sufficient evidence to support the single factor theory. A second EFA, requiring the model to extract only one factor, was conducted.

The second EFA, which was composed of a single factor, explained $25.79 \%$ of the variance in the six items. All six items loaded on to the factor, Behavioral PE, saliently, and factor loadings were somewhat variable (.35 to .72; see Table 10$)$. In addition to variability in the factor loadings, the residual correlation matrix indicated that there were several discrepancies between the reproduced and sample correlation matrices. Seven of the 15 residuals exceeded |.10|. Despite these discrepancies, the unidimensional model was retained and was then subjected to a CFA.

To confirm the results of the split-half EFA on Behavioral PE, a split-half CFA was conducted. The latent factor, Behavioral PE, explained all six of the original items and no residual terms were specified as correlated in the initial model (see Figure 7). This model reflected the Behavioral PE factor model from the original, three-factor CFA. This model was over-identified $(d f=9)$, and the path between Behavioral PE and item A1 was constrained to 1.0. Estimation of model parameters was conducted using FIML. 
The initial model, with all six items, did not fit the data well. The chi-square goodness of fit statistic was significant, $\chi^{2}(9)=80.66, p<.001$, indicative of lack of exact model fit. Additionally, the CFI (.63) was well below the standard cutoff around .95 , and RMSEA (.17) was above the .10 cutoff for an unacceptable model fit. The standardized residual covariance matrix indicated several extreme residuals (greater than $|2.0|)$, signifying serious discrepancies between the sample and model implied covariance matrices. Standardized factor loadings of the initial model varied, ranging from .26 (item I2) to .77 (item A1). All items had relatively high estimated residual variances with the exception of item A1.

Due to poor initial model fit, modification indices were explored. Based on modification indices, the model was respecified three separate times, allowing the error variances between items $\mathrm{C} 1$ and $\mathrm{C} 2$ (first), I1 and I2 (second), and C2 and I2 (third) to covary. Items I1 and I2 (i.e., involvement) and items C1 and C2 (i.e., coping behavior) were allowed to covary because it was deemed plausible that there was a concept related to involvement and coping behavior that these pairs of items measured that is different from Behavioral PE. Items $\mathrm{C} 1$ and $\mathrm{I} 2$ were allowed to covary because it was determined that the item wording might have tapped into a construct related to independence.

This model, which included all six items and allowed items C1 and C2, I1 and I2, and $\mathrm{C} 1$ and $\mathrm{I} 2$ to covary, was found to fit the data poorly. Although the chi-square goodness of fit statistic was significant, $\chi^{2}(6)=16.38, p=.01$, the fit indices suggested adequate fit $(\mathrm{CFI}=.95$ and $\mathrm{RMSEA}=.08)$. Despite the appearance of adequate model fit to the data according to the fit indices, the model was determined to fit poorly because of discrepancies between the sample and model implied covariance matrices. Table 23 
illustrates poor model fit, where several serious discrepancies between covariance matrices were found in the standardized residual covariance matrix. Additionally, two of the standardized factor loadings remained low (items I2 and C2; see Table 11), while the standardized factor loading for item A1 was the highest (.89). The estimated standardized measurement error variances reflected this trend (see Table 11). In total, these results suggested that item A1 was driving the factor, Behavioral PE.

This conclusion was also reached when items $\mathrm{I} 2$ and $\mathrm{C} 2$, which had low standardized factor loadings and high estimated standardized measurement error variances in the initial model, were eliminated from the model. In fact, this model was found to be non-positive definite. Non-positive definiteness was most likely due to the small, negative residual variance for item A1 (-.05). Therefore, no model for Behavioral PE was supported in the living unit setting. Refer to Figure 8 for the final model of Behavioral PE.

Behavioral PE in Treatment. The model for Behavioral PE did not fit the data well in the living unit setting; however, the fit nearly reached adequacy. This model (see Figure 8) was subsequently tested in the treatment setting. This was done in order to compare model fit of PE between the three settings. As in the CFA on Behavioral PE in the living unit, all six of the original indicators were included in the model and items I1 and $\mathrm{I} 2$, items $\mathrm{C} 1$ and $\mathrm{C} 2$, and items $\mathrm{I} 2$ and $\mathrm{C} 1$ were allowed to covary.

Similar to the single factor model for Behavioral PE in the living unit, the model for Behavioral PE in the treatment setting was found to have borderline adequate fit. Although the chi-square goodness of fit statistic was significant, $\chi^{2}(6)=19.92, p=.003$, the CFI was close to the standard cutoff for adequate model fit $(\mathrm{CFI}=.94)$ and the 
RMSEA (.09) was below the common cutoff for (un)acceptable fit (.10; see Table 12). Despite fit indices, an inspection of the standardized residual covariance matrix, which can be found in Table 23, resulted in support for insufficient model fit. There were six residuals greater than $|2.0|$, suggesting serious discrepancies between the sample and model implied covariance matrices for several items. Parameter estimates were assessed to confirm that the model was interpretable (see Table 11). Standardized factor loadings for the six indicators were all greater than .30 ; however, there was variability in the factor loadings, ranging from $.37(\mathrm{C} 2)$ to .75 (A1). As a result of this variability and as a result of discrepancies in the standardized residual covariance matrix, the model was determined to fit the data unsatisfactorily.

Behavioral PE in School or Vocation. Finally, the single factor model for Behavioral PE (see Figure 8) was examined in the school or vocational setting. The model for Behavioral PE was found to fit the data poorly (see Table 12). The chi-square goodness of fit statistic was significant, $\chi^{2}(6)=38.33, p<.001$, and the fit indices were poor $(\mathrm{CFI}=.86$ and $\mathrm{RMSEA}=.14)$. Upon inspection of the standardized residual covariance matrix, it was determined that the implied covariance matrix did not replicate the sample covariance matrix well (see Table 23). Evidence supported the notion that item A1 drove the Behavioral PE factor. As illustrated in Table 11, standardized factor loadings for items $\mathrm{C} 1$ and $\mathrm{C} 2$ were low. In fact, the standardized factor loading for item C2 was nearly zero. There was also a large discrepancy between the highest (.89 for item A1) and second highest (.47 for item I1) standardized factor loading. Furthermore, it was determined that the model for Behavioral PE did not fit the data well in the school or vocational setting. 
Validity of Behavioral PE

Comparisons of Behavioral PE Model Fit Across Settings. The model fit of Behavioral PE was compared across the three settings. Behavioral PE was found to be just shy of adequacy in the living unit and treatment settings. In contrast, the model more clearly did not fit the data well in the school or vocational setting. Although item A1 was found to drive the factor in all settings, distinct model specifications would have made the models fit the data better in the treatment and school or vocational settings. In these settings, other items, apart from item A1, were also strongly related to the factor. These differences were suggestive that the operationalization of Behavioral PE did not align across the three settings.

Predicting Behavioral success from Intrapersonal PE

Hypotheses $2 \mathrm{a}$ to $2 \mathrm{f}$ anticipated that Intrapersonal PE in certain settings would predict behavioral success in five OYA domains. These hypotheses were stated as the following:

$H_{2 a}$ : Controlling for age, total time incarcerated, and indeterminate versus determinate sentencing, psychological empowerment in school or vocational activities will positively predict behavioral success in the educational domain. $H_{2 b}$ : Controlling for age, total time incarcerated, and indeterminate versus determinate sentencing, psychological empowerment in school or vocational activities will positively predict behavioral success in the vocational domain. $H_{2 c}$ : Controlling for age, total time incarcerated, and indeterminate versus determinate sentencing, psychological empowerment in the youth's treatment group(s) will positively predict behavioral success in offense-specific domain. 
$H_{2 d}:$ Controlling for age, total time incarcerated, and indeterminate versus determinate sentencing, psychological empowerment in the youth's treatment group (s) will positively predict behavioral success in the mental health domain. $H_{2 e}:$ Controlling for age, total time incarcerated, and indeterminate versus determinate sentencing, psychological empowerment in the youth's treatment group(s) will positively predict behavioral success in the life/social skills domain. $H_{2 f}$ : Controlling for age, total time incarcerated, and indeterminate versus determinate sentencing, psychological empowerment in the living unit in which they reside will positively predict behavioral success in the life/social skills domain.

In order to assess Hypotheses 2a through $2 \mathrm{f}$ five hierarchical linear models (HLM) were conducted. One model for each of the five OYA-specified behavioral domains (i.e., educational or ED, vocational or VOC, offense-specific or OFF, mental health or MH, life/social skills or LSS) was assessed. HLM was conducted in order to account for nesting within living units. Each model allowed only the intercept to vary between units (i.e., random intercepts model). In total, 22 living units were used as the grouping variable. Within each unit, the number of youth participants ranged from one to 25. Three control variables were included in the analyses: (1) age (AGE); (2) total time incarcerated (TOTAL_TIME); and (3) commitment disposition (DOC) which was a proxy for indeterminate (i.e., OYA) versus determinate sentencing (i.e., DOC). Intrapersonal psychological empowerment in each of the three settings (i.e., school/vocational activities or VOC_INTRA, treatment groups or TREAT_INTRA, and the living unit or LIVE_INTRA) was also included in the appropriate model (i.e., 
depending on the hypothesis). All continuous predictors at level 1 (i.e., individual level) were grand mean centered. There were no variables at level 2 (i.e., living unit level); therefore, variability in the mean for each behavioral outcome between living units could not be further explained. The following random intercepts models were used to regress behavioral success within each of the five domains on the three control variables and Intrapersonal PE, controlling for living unit:

$$
\begin{aligned}
& \mathrm{ED}_{\mathrm{ij}}=\gamma_{00}+\gamma_{10}(\mathrm{AGE})+\gamma_{20}(\text { TOTAL_TIME })+\gamma_{30}(\mathrm{DOC})+\gamma_{40}(\text { VOC_INTRA })_{\mathrm{ij}}+ \\
& \mathrm{u}_{0 \mathrm{j}}+e_{\mathrm{ij}} \\
& \mathrm{VOC}_{\mathrm{ij}}=\gamma_{00}+\gamma_{10}(\mathrm{AGE})+\gamma_{20}\left(\mathrm{TOTAL} \_\mathrm{TIME}\right)+\gamma_{30}(\mathrm{DOC})+\gamma_{40}\left(\mathrm{VOC}_{-} \text {INTRA }\right)_{\mathrm{ij}}+ \\
& \mathrm{u}_{0 \mathrm{j}}+e_{\mathrm{ij}} \\
& \mathrm{OFF}_{\mathrm{ij}}=\gamma_{00}+\gamma_{10}(\mathrm{AGE})+\gamma_{20}(\text { TOTAL_TIME })+\gamma_{30}(\mathrm{DOC})+\gamma_{40}(\text { TREAT_INTRA })_{\mathrm{ij}} \\
& +\mathrm{u}_{0 \mathrm{j}}+e_{\mathrm{ij}} \\
& \mathrm{MH}_{\mathrm{ij}}=\gamma_{00}+\gamma_{10}(\mathrm{AGE})+\gamma_{20}(\text { TOTAL_TIME })+\gamma_{30}(\mathrm{DOC})+\gamma_{40}(\text { TREAT_INTRA })_{\mathrm{ij}} \\
& +\mathrm{u}_{0 \mathrm{j}}+e_{\mathrm{ij}} \\
& \mathrm{LSS}_{\mathrm{ij}}=\gamma_{00}+\gamma_{10}(\mathrm{AGE})+\gamma_{20}(\text { TOTAL_TIME })+\gamma_{30}(\mathrm{DOC})+\gamma_{40}(\text { TREAT_INTRA })_{\mathrm{ij}} \\
& +\gamma_{50}(\text { LIVE_INTRA })_{\mathrm{ij}}+\mathrm{u}_{0 \mathrm{j}}+e_{\mathrm{ij}}
\end{aligned}
$$

A summary of the results of the five HLM analyses can be found in Table 24 .

The grouping variable, living unit, accounted for a large percentage of the variability in behavioral success in each domain, ranging from 35 to $45 \%$. For comparison purposes, ICCs for facility-level differences in behavioral success were calculated. Note that facilities accounted for half the amount of variability in behavioral success than did living units. This is likely due to rater-biases, which was a confounding variable in this analysis (for further discussion see section on Limitations). 
Two of the control variables, commitment disposition and age, significantly predicted behavioral success in each of the five domains. While controlling for nesting within living units and all other predictors, commitment disposition was significantly and positively related to behavioral success in each domain such that DOC youth reported significantly higher behavioral success. Age was also consistently significantly related to behavioral outcome scores in each domain, where the mean age positively predicted behavioral success, controlling for nesting within living units and all other predictors.

The variable of interest, Intrapersonal PE, significantly predicted behavioral success in two of the five domains. Intrapersonal PE in the treatment setting was significantly related to the offense-specific domain, $t(355.50)=2.57, p=.01$, when controlling for nesting within living units, age, total time incarcerated, and treatment disposition. Offense-specific behavioral success scores were positively predicted by mean scores of Intrapersonal PE in the treatment setting. Although Intrapersonal PE in the treatment setting significantly predicted offense-related behavioral success controlling for all other variables, it did not significantly predict mental health or life and social skills behavioral success, $t(191.04)=.95, p=.35$ and $t(359.04)=1.55, p=.12$, respectively. In addition to Intrapersonal PE in the treatment setting, Intrapersonal PE in the living unit was included in the random intercepts HLM predicting life and social skills behavioral success. Similar to effects found for the treatment settings, Intrapersonal PE in the living unit was not significantly related to behavioral success in this domain, $t(358.23)=.08, p=.93$, when controlling for age, total time incarcerated, commitment disposition, Intrapersonal PE in the treatment settings, and living units. 
Comparable to findings related to Intrapersonal PE in the treatment setting, Intrapersonal PE in school or vocational activities significantly predicted behavioral success in only one of two domains. Controlling for all other variables and nesting within living units, Intrapersonal PE in school or vocational activities significantly predicted behavioral success in the mental health domain, $t(251.28)=2.07, p=.40$. Holding all else constant, there was a positive relationship between mental health behavioral success and mean Intrapersonal PE scores in school or vocational activities. In contrast, Intrapersonal PE in school or vocational activities did not significantly predict educational behavioral success when controlling for age, total time incarcerated, commitment disposition, and nesting within living units, $t(325.92)=.97, p=.34$.

It should also be noted that there was significant variability in mean behavioral success scores between living units across all five domains. As will be mentioned in Chapter IX, if level two variables were available, additional models could be run in order to account for some of this variability. Within these analyses, there still remained a portion of variability in behavioral success scores between living units that was unaccounted for. 


\section{CHAPTER IX}

\section{Discussion}

This study was the first of its kind to extend literature on psychological empowerment into a youth correctional context. This research took initial steps to understand the factor structure of psychological empowerment as well as its potential behavioral benefits in this context. While this research did not support the first set of hypotheses related to the factor structure and only partially supported the second set of hypotheses, this study contributes to the literature on psychological empowerment and the juvenile justice system. In light of the call for research on new and innovative strengths-based programming for incarcerated youth (Andres-Hyman, Forrester, AcharaAbrahams, Lauricella, \& Rowe, 2007; Bazemore \& Erbe, 2003; Bazemore \& Terry, 1997; Butts, Mayer, \& Ruth, 2005; Marshall et al, 2005; Wormith et al., 2007), this study was the first of its kind to attempt to establish a measure of empowerment to be used in future research within a youth correctional setting. As a result of the measurement development findings, there were several potentially important future directions that can be gleaned from this study.

This chapter will first briefly recapitulate the study findings under each set of hypotheses. Relevant literature will be reviewed to place findings in the larger context of empirical work on the topic. Within this examination, several important limitations and future research directions will be briefly highlighted. A more thorough analysis of the potential study limitations will follow. Limitations regarding both sets of hypotheses will be considered. Finally, this chapter will conclude with an in-depth discussion of the 
strengths, implications, and future directions, integrating the findings and limitations of the present study to justify the call for further research on this topic.

\section{Confirmatory Factor Analysis on the Correlated Three-factor Psychological}

\section{Empowerment Model}

The following section will recapitulate the study findings related to the factor structure of the psychological empowerment scale in the three correctional settings. In doing so, it will integrate research literature on empowerment to help explain the results. This section will parallel the Results chapter, where the three-factor structure will first be discussed, followed by results of the analyses conducted to explore the factor structure of each component. Embedded within the appropriate sub-sections, findings related reliability and validity of the sub-scales, including measurement invariance, internal consistency, and discriminant validity, will be interpreted.

The Three-Factor Structure of Psychological Empowerment. It was originally proposed that three factors would underlie psychological empowerment in each of the three settings. This structure was theoretically supported in the literature on psychological empowerment (Zimmerman, 1995; Zimmerman \& Warchausky, 1998). Leading researchers assert that psychological empowerment is composed of three principal components: (1) intrapersonal; (2) interactional; and (3) behavioral empowerment (Zimmerman, 1995; Zimmerman \& Warchausky, 1998). It has been argued that these three components cover the domain breadth of psychological empowerment as they address an individual's motivational and perceived ability to influence his or her circumstances (i.e., intrapersonal psychological empowerment), an individual's actual participation in creating desired change (i.e., behavioral), and an 
individual's critical reflection on environmental factors that impact his or her circumstances as well as knowledge of resources that are available to him or her to create desired change (i.e., interactional; Zimmerman, 1995; Zimmerman \& Warchausky, 1998).

This three-factor model, which has dominated the literature on psychological empowerment for over a decade, was not supported by data from this study. While CFAs on the three-factor structure resulted in non-positive definiteness, inter-item correlation matrices in each of the correctional settings (i.e., living unit, treatment, school or vocation) indicated that there were some potentially serious issues amongst the items, including low and negative correlations. Thus, it was concluded that some items did not represent the theoretical components of psychological empowerment well. In order to further explore dimensionality underlying the three components of psychological empowerment (i.e., Intrapersonal, Interactional, Behavioral), three split-half EFAs followed by split-half CFAs were conducted.

\section{Confirmatory Analyses on the Factor Structure of Intrapersonal PE}

Split-half EFA/CFAs on Intrapersonal PE. The split-half EFAs on Intrapersonal $\mathrm{PE}$ in the living unit supported the notion of unidimensionality. Item PC2, which was the negatively worded item under the perceived control domain, was eliminated from the Intrapersonal component. This item was only slightly revised from the original item. The scale from which this item was adapted, which measured perceived constraints, was originally developed for use within a general population of adults ages 25 to 75 (Lachman \& Weaver, 1998). It is possible that youth did not understand this item as it required them to respond to an indirect or negatively worded stem. This scale was also 
developed for use within a general, non-criminal population, and the way in which the items are perceived might be different within a correctional environment. After the final items were identified for each of the two PE components in the living unit setting, the factor structures were confirmed through split-half CFAs.

A split-half CFA on Intrapersonal PE resulted in a single-factor model, consisting of four indicators, and was determined to fit the data well in the living unit setting. In addition to item PC2, which was eliminated as a result of the split-half EFA, item MC2 was deleted from the model in the CFA. This item was negatively worded, and it is likely that youth found it to be confusing. Anecdotally, several youth questioned the meaning of the key phrase within the item, "take a back seat." This idiom might not have been commonly known to some of the youth, particularly the younger participants. The final model fit the data well, and results in the other two settings (i.e., treatment and school or vocational activities) were substantively the same. These findings justified the use of the sub-scale's average composite score to predict behavioral success (i.e., Hypotheses $\left._{2 \mathrm{a}-\mathrm{f}}\right)$. Beyond this study, the four items within this sub-scale can be used as a foundation for measuring Intrapersonal PE in the living unit, treatment groups, and school or vocational settings.

Measurement Invariance. Results comparing the factor structure of Intrapersonal PE between survey administrations were mixed. In the living unit and school or vocational settings, results revealed that the single-factor model with no constraints (i.e., Step 1) was unsatisfactory (i.e., non-positive definite) in online and paper-and-pencil survey formats. Model misfit was not anticipated as the single-factor model was shown to be a good fit within the split-half sample, as a whole. However, when further dividing 
the sub-sample into two groups based on survey administration, the models no longer fit well. While it is possible that the survey formats (i.e., online and paper-and-pencil) did not measure the construct in a similar manner, it is more likely that the sub-sample sizes, ranging from 94 to 179 , were smaller than the optimal sample size for CFA. It is assumed that CFA is a large sample technique, thus sample sizes less than 250 might result in poor model fit (Wegener \& Fabrigar, 2000). To further investigate these discrepancies, multi-group CFAs could be conducted with the full sample population, which would include over 500 youth responses.

In contrast, results of the CFAs examining measurement invariance in the treatment setting supported the notion that the survey formats elicited similar responses to items under the Intrapersonal PE sub-scale. The most stringent model, which constrained the factor loadings, intercepts, and residual variances to equivalence across survey administrations, was not significantly different than the model with no constraints. Therefore, it was concluded that there was no statistical difference in the way in which the online survey format and the paper-and-pencil survey format measured Intrapersonal $\mathrm{PE}$ in the treatment setting. This conclusion was supported by the practical insignificance of the difference in means of Intrapersonal PE. It was originally expected that the operationalization of Intrapersonal PE, or psychological empowerment more generally, would be affected by the method of survey administration. Although no studies on measurement equivalence of psychological empowerment in different survey administrations were found, empirical evidence has suggested that self-efficacy, a primary domain of Intrapersonal PE, is measured comparably in online and on paperbased self-report surveys (Schwarzer, Mueller, \& Greenglass, 1999). In combination 
with limited evidence within the research literature, these results support the notion that survey administration does not statistically impact the measurement of Intrapersonal PE in the treatment setting.

The invariance of measure administration may be called into questioned when contrasting CFA findings across the three settings. In the treatment setting, it was evident that there was no statistical difference in factor structure between survey formats. Results in the living unit and school or vocational activities did not support this conclusion. It is plausible that there was something about the way in which the scale assessed psychological empowerment in the living unit or school or vocational activities that resulted in dissimilar models between the two administrations. The explanation that youth were more engaged in the survey in the online version compared to the paper survey might be more likely than the latter explanation (i.e., construct is different between survey formats). This was the first time that OYA offered an online survey format to youth, and the opportunity to use the computer might have been more interesting to them compared to the paper version. In support of this observation, it took less time for youth to complete the survey online than on paper. In addition to differences in completion time, which ranged from 25 to 40 minutes, it is possible that fatigue or disinterest might have caused some of the discrepancies found within the measure invariance analyses in the living unit and school or vocational activities settings. Reliability of Intrapersonal PE

The internal consistency for Intrapersonal PE was unacceptable in all three settings. This was not surprising as Cronbach's alpha is a function of, among other things, number of items contained in the scale (Cronbach, 1951). As such, internal 
consistency was likely low due to the small number items $(N=4)$ in the sub-scale. While the four items that were used to measure Intrapersonal PE in the current study set the groundwork for assessing this construct, expanding this sub-scale to include more items will help increase its reliability. In particular, items related to the motivation to control and the perceived control domains, which each lost the negatively worded item, should be added to increase Cronbach's alpha.

\section{Validity of Intrapersonal PE}

Comparisons of Intrapersonal PE Model Fit Across Settings. It was originally expected that psychological empowerment within the youth correctional context could be operationalized similarly across settings because these settings are all housed within the same general context, the youth correctional facility. The results from the Intrapersonal PE scale generally supported this expectation, with the exception of a discrepancy between items $\mathrm{MC} 1$ and PC1 in the school or vocational setting. In the school or vocational setting, slight modifications to the specification of Intrapersonal PE might fit the data better. Despite this slight difference, the items measuring Intrapersonal PE serve as a foundation to the operationalization of the construct across the three settings in this context.

Discriminant Validity of Intrapersonal PE. Intrapersonal PE was found to have discriminant validity within this study. Intrapersonal PE was most highly correlated with the measure of locus of control; however, this correlation was only moderate, suggesting that these constructs were distinct. This result is consistent with previous theoretical and empirical research (Wallerstein, 1992; Zimmerman, 1990). Locus of control has been identified as a personality characteristic that often drives perceived control (Rotter, 
1966). Individuals that typically attribute life changes to internal characteristics rather than external factors are more likely to perceive that they have the power and capability to make changes in their lives (Rotter, 1966). Perceived control is a key element in Intrapersonal PE (Zimmerman, 1995; Zimmerman \& Warschausky, 1998), thus, individuals with a high degree of locus of control are likely to report high scores on Intrapersonal PE. Fostering a sense of control within one's own life, particularly for those youth that are predisposed to attributing control to personal characteristics, is likely to increase Intrapersonal PE overall.

Intrapersonal PE was also found to relate to the HCCQ, which measured feelings of autonomy in treatment. As expected, out of all three settings, Intrapersonal PE in the treatment setting had the highest correlation with HCCQ. The positive relationship between autonomy and Intrapersonal PE align with previous research findings. Empirical work on this topic has demonstrated a strong link between autonomy and empowerment, particularly within the organizational literature (DeSisto \& DeSisto, 2004; Parker \& Ohly, 2008; Pearson \& Moomaw, 2005; Seibert, Silver, \& Randolf, 2004). Additionally, constructs that are comprised by Intrapersonal PE (e.g., self-efficacy, perceived control) have also been positively associated with autonomy (Evans \& Fischer, 1992; Garcia \& Pintrich, 1996; Ng, Ang, \& Chan, 2008; Wang \& Netemeyer, 2002). Furthermore, programming that provides youth with autonomy to accomplish their goals might also increase their Intrapersonal PE.

Correlations between Intrapersonal PE and the sub-scales of the HPES were also examined. There was variability in the correlations, from modest to high. Across settings, Intrapersonal PE was generally most correlated with the Activity, Growth, and 
Support sub-scales. Moderate to high correlations between these sub-scales and Intrapersonal PE were to be expected as work on empowering settings has suggested that empowerment is best achieved when the environment provides core activities that are engaging and meaningful, space for personal growth, and egalitarian support for all of its members (Maton, 2008; Maton \& Salem, 1995). Additionally, research has found that adults play an important role in facilitating youth empowerment by creating a structured, safe atmosphere where youth can interact, take on new roles and responsibilities, and receive constructive, positive feedback about their work (Cargo et al., 2003; Messias et al., 2005). This evidence is suggestive that programming that provides a positive, supportive, active environment is likely associated with an increase in youths' experiences of Intrapersonal PE.

While Intrapersonal PE was associated with, yet distinct from these measures of related constructs, interpretations of discriminant validity should be made with caution. As discussed more thoroughly in the Limitations section, these scales were revised by researchers at OYA. For this reason, discriminant validity is restricted by compromised construct validity of the associated measures (i.e., LOC, HPES, HCCQ).

\section{Confirmatory Analyses on the Factor Structure of Interactional PE}

Split-half EFA on Interactional PE. As anticipated, due to poor inter-item correlations amongst items in the Interactional component, the split-half EFA on Interactional PE in the living unit did not reveal an interpretable model for further investigation. Thus it was concluded that these six items did not "hang together well." It might be the case that the three domains that were selected (i.e., awareness of resources, critical awareness, problem-solving) did not represent the Interactional component of PE; 
however, it is more likely that items did not sufficiently capture this domain. Of particular concern were items CA1 and CA2 (i.e., critical awareness domain), which correlated negatively with many of the other items within Interactional PE. No previously validated scales measured critical awareness in a way that could be easily adapted to the youth correctional context. Thus, these items were created based on one item, which was positively worded, measuring critical awareness within the Social Worker Empowerment Scale (Frans, 1993). The items developed for the youth correctional context might not have reflected the meaning of Frans' (1993) item well. For these reasons, items CA1 and CA2 might have been confusing for the participating young men. This explanation was supported by the fact that the meaning of these two items was frequently asked about during the data collection process.

Items CA1 and CA2 were not as closely related to one another as originally anticipated. Item CA1 referred to critical awareness related to the influence of other youth, while item CA2 attempted to capture beliefs about the influence of OYA staff on behavior. It was intended for these two items to be at least moderately correlated, suggesting that they measured something in common. Despite this presumption, the correlation between these items was close to zero $(r=-.01)$. One explanation for this result is that it is likely that youth perceived the influence of peers and staff differently. It was originally intended for these items to measure the same concept. For this reason, it will be useful for future research to be consistent in specifying the subject of the item. In other words, instead of asking participants about staff and peer influences, expecting these items to be highly correlated, items related either to the influence of peers or related to the influence of staff should be used to measure critical awareness. Other influential 
factors at higher ecological levels (e.g., policies) could also be considered as the subject of the item.

Similar to problems developing items measuring critical awareness, difficulties in item selection across the Interactional component of PE negatively impacted the subscale. The operationalization of the interactional component of psychological empowerment is least consistently defined within the research literature. Of the nine psychological empowerment measures examined, less than $50 \%$ implicitly or explicitly measured interactional PE (Akey et al., 2000; Holden et al., 2005; Speer \& Peterson, 2000; Zimmerman \& Zahniser, 1991). To the knowledge of the researcher, these items were used in only the second attempt at measuring the interactional component of PE within a population of young adults. These findings are evidence that items within the Interactional sub-scale need to be articulated better and more concisely. Before solidifying the articulation of each item, however, Interactional PE should be better conceptualized within a young (incarcerated) adult population.

\section{Confirmatory Analyses on the Factor Structure of Behavioral PE}

Split-half EFA/CFA on Behavioral PE. Unidimensionality of Behavioral PE in the living unit was supported through split-half EFAs. The EFA suggested that all items should be retained within the model. All items were then entered into confirmatory analyses.

The single-factor model for Behavioral PE included all six of the original subscale items and did not fit the data well for the living unit setting. Item A1 carried the majority of the weight of Behavioral PE factor in the living unit. In other words, out of all the items used in this sub-scale, the directly stemmed or positively worded item under 
the advocacy domain (i.e., A1) best measured Behavioral PE. It is possible that item A1, which utilized a direct stem, was most clearly worded, as it was the most concise item in the sub-scale. It is also likely that one's ability to advocate for himself was most closely related to the construct of Behavioral PE, where an individual that advocates for himself takes action to influence the social and political environment (Zimmerman \& Warchausky, 1998). The notion that advocacy is most closely associated with Behavioral PE is supported by the fact that item A2, which was the negatively worded item under this domain, was estimated to have the second highest standardized factor loading. Despite the finding that items related to advocacy might best measure Behavioral PE, the theoretical breadth of Behavioral PE also includes participation or involvement and coping behaviors (Zimmerman \& Warschausky, 1998).

The possibility that, despite evidence of unidimensionality in the EFA process, Behavioral PE is multidimensional should not be overlooked. The final model of Behavioral PE incorporated several correlated residual variances, including correlations between items $\mathrm{C} 1$ and $\mathrm{C} 2$ and items I1 and I2. These items correspond to the coping behaviors and involvement domains, respectively. Despite theoretical support for this domain breadth (Zimmerman, 1995; Zimmerman \& Warschausky, 1998), it is possible that the constructs selected to represent Behavioral PE do not have sufficient overlap to be considered as measuring a single construct. Therefore, the conceptualization of Behavioral PE should be re-examined.

As previously mentioned, the models for the Intrapersonal and Behavioral PE in the living unit included correlated residual or measurement error variances. In the Intrapersonal PE model, the residual variances for items SE1 and PC1 were allowed to 
covary, and in the Behavioral PE model, items I1 and I2, items $\mathrm{C} 1$ and $\mathrm{C} 2$, and items $\mathrm{C} 1$ and I2 were allowed to covary. Covariance of residual terms is indicative that another construct, unrelated to Intrapersonal or Behavioral PE, or measurement issues explained variability in the indicators. In the case of Behavioral PE, the association between residual terms for items I1 and I2 was assumed to be related to an aspect of involvement that was not explained by Behavioral PE. Similarly, it was assumed that items C1 and C2 captured an aspect of coping behavior that was unrelated to Behavioral PE. Items I1 and C2 were also allowed to covary in the final model, which was justified because it was plausible that these items also captured information about one's feelings of independence. As a result, the six items within the Behavioral PE sub-scale, particularly the items related to involvement and coping, did not measure Behavioral PE alone.

Reoperationalization of these items could help minimize the measurement error related to involvement and coping behavior.

Measurement error was also due, in part, to negatively worded items. Note that in both models, positively worded (items PC1 and C1) and negatively worded (SE1 and I2) item residuals correlated negatively ( $r=-.35$ and $r=-.24$, respectively). This finding suggested that direct and indirect stems might elicit opposite and related information that is not related to PE. Additionally, indirectly stemmed items were flagged as problematic within the EFA and CFA analyses, and, anecdotally, some youth participants asked questions about several negatively worded items (i.e., items MC2, AR2, I2) during data collection. Research has suggested that negatively worded items can be problematic or confusing for youth respondents (Corwyn, 2000; Schmitz \& Baer, 2001). More closely related to this study, negatively worded items have been found to cause poor model fit in 
other common operationalizations of psychological empowerment in previous research (Peterson et al., 2006; Zimmerman \& Zahniser, 1991). Within the empowerment context, it has been argued that negatively worded items might actually measure different constructs than their positively worded counterparts (Peterson et al., 2006). For these reasons, some survey researchers have suggested that all items should be positively worded and the response stem should be reversed at different points in the survey in order to identify response bias (Barnette, 2000).

\section{Validity of Behavioral PE}

Comparisons of Behavioral PE Model Fit Across Settings. In contrast to Intrapersonal PE model fit across settings, results suggested that Behavioral PE should be operationalized differently within each setting. In the research literature on empowerment, it is commonly noted that the construct is context dependent and should be conceptualized within the specific setting in which it is studied (Rappaport, 1987; Zimmerman, 1995). While the conceptualization of Intrapersonal PE might carry over from setting to setting within the correctional context, the way in which youth assert themselves, taking charge of their power-oriented goals (i.e., Behavioral PE), might be different between the living unit, treatment, and school or vocational settings. In the treatment and school settings, power-oriented goals, which are self-defined goals to gain the power to accomplish a desired outcome (Cattaneo \& Chapman, 2010), are often guided by the larger context (e.g., treatment plan, ground rules, lesson plans). In fact, these goals may not be self-defined at all. It is conceivable that youth might have more latitude to identify their own power-oriented goals in the living unit and vocational settings. Differences between goals and whether or not these goals are self-defined will 
impact the way in which youth enact them (Cattaneo \& Chapman, 2010). Furthermore, the conceptualization of Behavioral PE, which includes advocacy, involvement, and coping behaviors, is likely to look different in various contexts. For instance, in the living unit setting, a youth might be likely to advocate for changes in rules such as more recreation time based on good behavior. In the treatment setting, goals related to coping behavior, such as positively connecting with at least one staff member, might be more relevant. For this reason, it might be advisable to develop measures of Behavioral PE specific to each setting.

As an alternative to item specificity, it might also be possible to construct behavioral items that can apply universally to all contexts, even those outside of correctional settings. These items would need to refer to the self-identified poweroriented goals, which would inherently situate them within their own context. For example, an open-ended item asking youth to list the actions taken to achieve his goal in the last month could be sufficiently broad, so long as the youth identified a goal prior to responding. By selecting items that are either much more broad and refer to a particular goal or more specific might enable researchers to capture more information about the behaviors enacted within certain settings.

Predicting Behavioral success from Intrapersonal PE

The second set of hypotheses, predicting behavioral success from aspects of psychological empowerment, was partially supported (i.e., $\mathrm{H}_{2 \mathrm{~b}}$ and $\mathrm{H}_{2 \mathrm{c}}$ ). Intrapersonal PE significantly predicted behavioral success in two of the five OYA domains. Controlling for all other variables, including nesting within living units, mean Intrapersonal PE treatment scores were positively associated with offense-related 
behavioral success. There was also a positive relationship between mean Intrapersonal PE in school or vocational activities and behavioral success in vocational domain, holding all else constant.

Significant findings in the offense-specific and vocational domains reflect existing research literature on empowerment-based programming for youth. Although no research has been conducted on empowerment in a youth correctional setting, theoretical and empirical evidence indicates that youth who experience psychological empowerment are more likely to be engaged; actively participate; demonstrate behavioral control, conflict resolution, and collaboration; and create positive or constructive change (Cargo et al., 2003; Kim, Crutchfield, Williams, \& Hepler, 1998; Wilson, Minkler, Dasho, Wallerstein, \& Martin, 2008). Results that support the research hypotheses can contribute to the empirical literature by providing additional evidence of positive associations between behavioral indicators and Intrapersonal PE.

Non-significant results in the current investigation might be explained by the imperfect nature of the sub-scales used in this study. While the hypotheses predicting behavioral success from Intrapersonal PE were not supported in several OYA domains (i.e., educational, life and social skills, mental health), there were clear issues related to the reliability of psychological empowerment. As previously discussed, there was a large amount of measurement error, which impacted the scores of Intrapersonal PE as reflected in the low internal consistency estimates. In analyses on the Interactional component of psychological empowerment, dimensionality was not established. Similarly, single-factor structure of Behavioral PE was not supported. Composite scores representing Interactional and Behavioral PE were, therefore, not utilized in analyses predicting 
behavioral success. Thus the results in the current study predicting behavioral success from psychological empowerment as a broad construct were inconclusive. Further work on the operationalization and even conceptualization of each component of psychological empowerment, including Intrapersonal PE, in a youth correctional context is necessary. With improved scales, researchers will be better able to estimate the true relationship between behavioral success and psychological empowerment.

\section{Limitations of the Current Study}

Limitations in the present research impacted the conceptualization and operationalization of psychological empowerment as well as its ability to predict behavioral success. The following section will discuss limitations related to each of the sets of hypotheses. First, explanations for poor model fit will be discussed. Next, constraints on construct validity of psychological empowerment are assessed. Finally, limitations related to the second set of hypotheses, which examined associations between Intrapersonal PE and behavioral success, will be discussed in terms of design issues impacting internal validity and construct validity of the dependent variables.

\section{Limitations Related to the Factor Structure of Psychological Empowerment}

As previously mentioned, there were several issues that potentially caused the single-factor models to fit the data poorly. First, the indirectly stemmed or negatively worded items were likely confusing to some of the participants. Second, the operationalization of psychological empowerment, in particular Behavioral PE, was not consistent across correctional settings. Third, it is possible that responses varied depending on survey administration, although this result might also have been caused by small sub-sample sizes. Fourth, internal consistency of the supported sub-scale (i.e., 
Intrapersonal PE) was low in all three settings. These issues were detrimental to the factor structure of psychological empowerment, and as a result, construct validity of psychological empowerment was not established.

Beyond these issues, other limitations concerned the measurement of PE in different contexts, self-report bias, and false assumptions about power-oriented goals. First, the measurement of PE in three different contexts during a single data collection session might have been problematic. During data collection, it was apparent that many participating youth were confused by and frustrated with "repeating questions." The scales measuring psychological empowerment in the three settings were ordered consecutively within the survey. Thus, participants read what seemed to be the same set of 18 items three times in a row. The items were ordered differently within each scale; however, the repetitive nature of the items was apparent to the youth.

At the same time, the written instructions for each psychological empowerment scale specified, in bold typeface, to which correctional setting each set of items referred. In addition to the written instructions, a verbal warning was given to the youth during the introductory explanation of the annual survey made by the OYA representative. Despite attempts to make participants aware that sets of items were repeated but referred to three different contexts, many youth did not seem to understand this until they reached the second or third set of items (i.e., scales). This was made apparent by the number of questions and side comments made about these scales. Additionally, it is likely that not all participants that were confused or frustrated voiced their concerns, suggesting that the measurement of psychological empowerment in different settings, and especially presenting the scales consecutively, posed a serious threat to the validity of each scale. 
Despite the anecdotal evidence that this was an issue during survey

administration, the correlations between Intrapersonal PE in the three settings were not so high as to assume that they measured the exact same construct or that youth responded to the items in each setting in exactly the same way. If youth did not realize that each scale was presented within a different setting, it would have been expected that the correlations between the Intrapersonal sub-scales in the three settings were higher (e.g., $r \geq .85$ ). Correlations near 1.0 would have indicated that the participants responded to the items nearly identically across the three settings. Although the magnitude of the relationships between Intrapersonal PE in the three settings was within an acceptable range, allowing for the conclusion that they were distinct, it is still possible that participants were fatigued by the time that they reached the end of the second and third scales measuring psychological empowerment. Fatigue might have seriously contributed to their difficulty in understanding that the three sets of items referred to different contexts.

The factor structure of psychological empowerment might have also been influenced by use of self-report data. For example, some participants' perceptions of socially desirable answers may have influenced their responses. Another issue related to self-report is that some youth might have an unrealistic or inflated perspective of their ability to change or control their environment. For this reason, the self-report measures of psychological empowerment could have elicited dishonest responses, particularly in light of the fact that data was not collected anonymously. Ratings on psychological empowerment from other perspectives (e.g., OYA staff) were not collected; thus, triangulation of scores on psychological empowerment was not made possible. Researchers have, however, suggested that self-report questionnaires can obtain equally 
valid or more valid information from incarcerated youth compared to interviews, official reports, or other methodological procedures (Kaufman, Hilliker, Lathrop, Daleiden, \& Rudy, 1996; Krohn, Waldo, \& Chiricos, 1974; Elliott \& Ageton, 1980). Self-report was utilized because it was the most appropriate method to access personal information (e.g., perceived control, self-efficacy, perceived competence) about the youth. Ratings related to behavioral success, which might be more affected by self-report rater bias, were measured from an external perspective, eliminating the potential impact of self-report bias in the second set of hypotheses. While literature suggests that self-report data can be reliable within incarcerated populations, the factor structure of psychological empowerment was constrained. Biased responses impacted the model to the extent that measurement error was introduced.

Another limitation affecting the hypothesized model was that the conceptualization of psychological empowerment was predicated on an assumption that might have been incorrect for some youth. Underlying psychological empowerment is the notion that youth had established and were working toward meeting their poweroriented goals (Cattaneo \& Chapmna, 2010). According to Cattaneo and Chapman (2010), a power-oriented goal is a goal that is self-defined, meaningful to the goal-seeker, and relates to influencing social interactions at the dyadic, institutional, community, or societal level. A youth's power-oriented goal in the living unit setting might look completely different than his goal in the treatment setting. In addition to these contextual differences, underlying the conceptualization of psychological empowerment is the assumption that youth already established power-oriented goals. It is entirely possible that some youth had yet to define or were uninterested in defining power-oriented goals 
in certain contexts or in all contexts, for that matter. Another possibility is that poweroriented goals in the treatment setting, for example, were not self-defined. Instead, treatment related power-oriented goals might be defined by the treatment plan created by OYA staff or dictated by the treatment modules in which a youth participates. When power-oriented goals are self-defined, there is an intrinsic motivation to work toward meeting these goals, thus the youth is more likely to actively engage in the empowerment process (Cattaneo \& Chapman, 2010). Externally defined goals, however, might not inspire a youth to actively engage in this process.

The definition of and extent to which youth have established power-oriented goals was not assessed in this research; thus the operationalization of psychological empowerment was further limited. It should not be assumed that youth were working toward some power-oriented goal. Although the concept of psychological empowerment implies that a power differential exists and that participants actively seek to minimize that differential, the directions did not instruct participants to think of a goal for each setting. In this study, it is impossible to conclude which goals, if any, youth were thinking about when responding to the items within each correctional setting. It is even unknown whether or not a youth had anything to advocate for in the first place. An example of this might be that a youth that is complacent about rules in the living unit is not likely to want to advocate for change. Asking questions related to advocacy would be negligible in the case that a youth was uninterested or had nothing for which to campaign. Without having determined whether or not youth had identified a power-oriented goal in each setting, the operationalization of psychological empowerment was incomplete because psychological 
empowerment cannot be fully understood without knowing whether or not youth have identified some kind of desire for change.

\section{Limitations Related to Construct Validity of Psychological Empowerment}

Several issues impacted the extent to which construct validity of psychological empowerment could be assessed. These issues appertain to discriminant validity, facility-level factors, antecedents of psychological empowerment, and its negative effects. Future research that better addresses these limitations will have more evidence to support claims of construct validity.

By using revised versions of validated scales, discriminant validity of Intrapersonal PE was compromised. Three scales that were included in the OYA Annual Survey were used to assess discriminant validity of Intrapersonal PE (i.e., HPES, LOC, HCCQ). While Intrapersonal PE was related yet distinct from each of these three scales as well as sub-scales within the HPES, OYA adapted them, to varying degrees, from previous measures. Item wording within the LOC was only slightly modified in order to simplify language. In contrast to the LOC scale, the HCCQ and HPES were more drastically revised. Items within the HCCQ were adapted in order to simplify language as well as fit the correctional treatment context. The HPES was developed as a new measure of attitudes related to the prison environment based on two different prison environment questionnaires. Thus, the HPES was subject to changes in domains represented by the measure as well as changes to individual items. Without (re)validation of these scales, a thorough discussion of discriminant validity of Intrapersonal PE was limited. Although these scales had not been validated, it should be noted that the internal consistency of these scales was generally within the "acceptable" range or better. With 
additional validation work, it is expected that the relationships between Intrapersonal PE and prison environment, locus of control, and health care climate would reflect the relationships found in the present research.

Between facility differences in demographic composition might have also limited construct validity of Intrapersonal PE in two specific ways. First, it was not anticipated that the structure, environment, or demographic characteristics of youth at each facility would impact the operationalization of psychological empowerment. For this reason, no statistical measures were taken within confirmatory factor analyses to account for facility-level differences. This assumption might have been false, and construct validity of Intrapersonal PE is limited to the extent that facility characteristics shaped the definition of Intrapersonal PE in each facility. Second, there were significant between facility differences on four key demographic variables (i.e., age, total time incarcerated, commitment disposition, ethnicity; see Appendix C). These differences could be used to further explore the validity of the sub-scale. Cases where Intrapersonal PE was higher in facilities with older youth, for example, would support its construct validity. Unfortunately, the Intrapersonal PE sub-scale was inadequately operationalized, and additional analyses exploring these differences were not conducted. Once a defendable sub-scale and an overall psychological empowerment scale are established, these differences should be revisited in order to establish criterion-groups validity.

The incomplete understanding of the development and experience of empowerment, including negative experiences related to psychological empowerment, also impacted construct validity. First, antecedents such as individual-level and contextual factors, which impact youths' experiences of psychological empowerment in 
OYA correctional and re-entry facilities, were not thoroughly explored. Individual-level factors that may contribute to psychological empowerment in incarcerated youth include tag level (i.e., privileges allowed based on behavior), minority status, and anticipated or actual time remaining in incarceration. Beyond the individual-level, it is well known that the process and experience of empowerment is impacted by contextual factors, including a group-based belief system, opportunity role structure, a relational and welcoming environment, and positive youth-adult relationships (Cargo et al, 2003; Maton, 2008; Maton \& Salem, 1995, Messias et al, 2005). Some contextual factors that might impact youth empowerment specific to this setting include proportion of youth at certain tag levels, youth-adult relationships, a "cold" or unwelcoming environment, limited personal space or privacy, restricted access to buildings and events, limited contact with the community, staff empowerment, and problem-focused culture of the juvenile justice system. In support of the notion that contextual factors impact the experience of psychological empowerment, Schwartz (2000) has suggested that "jaded staff” members, or staff who were once passionate but burned out over time, have the potential to become barriers to positive youth development. It is possible that the environment (e.g., values, expectations, characteristics being modeled by staff) was not conducive to the experience of empowerment, and this type of observation was not possible under the present study design. Antecedents were not examined because foci of the present research related to the factor structure and potential outcomes of psychological empowerment in this unique context. While the relationship between some of these concepts and Intrapersonal PE was explored in order to investigate discriminant validity, a more thorough investigation of individual- and contextual-level factors is warranted. Future research should address 
these issues through statistical analyses that allow for both individual-level (e.g., minority status) and higher-level (e.g., staff empowerment) factors to be assessed through quantitative analysis or through in-depth qualitative research. This type of investigation would grant researchers the ability to establish the antecedents of psychological empowerment in this setting.

Despite this limitation, in order to control for some of the potential contextual impacts on youth psychological empowerment, this study included an examination of psychological empowerment within three different settings within the youth correctional and re-entry facilities. It was intended that this would allow for the comparison of reports of psychological empowerment across correctional settings more readily. While the literature encourages the measurement of psychological empowerment in specific contexts, doing so consecutively within the survey, as previously discussed, is not advisable.

Constraining predictive validity of psychological empowerment, this study did not investigate the potentially negative effects of empowerment. Although not explored in this dissertation, empowerment might result in negative outcomes, such as acting out or decreased self-efficacy. These outcomes might be more likely in the instance that contextual factors limit the degree to which young men in correctional facilities are able to demonstrate their empowered state. Believing that one is capable of influencing his environment yet being unable to do so might cause negative as opposed to positive intended effects (Cattaneo \& Chapman, 2010). For example, a youth who feels competent and confident in his skills, is able to reflect on environmental factors that impact him, and can work with other youth effectively, might attempt to create change to 
a rule that youth perceive as impacting them negatively (e.g., limited internet access). Given the restricted nature of the juvenile correctional setting, policy or rule change might be difficult or impossible for youth to initiate; therefore, youth may encounter a “glass ceiling” of sorts (L. Lichty, personal communication, March 21, 2012). This "glass ceiling" might be discouraging to youth and could foster anger, resentment, and acting out. Such an outcome is opposite the intended effect of programming designed to build self-esteem, self-efficacy, and confidence to initiate and participate in changerelated activities. It was not possible to fully understand potential negative effects on youth in this study because contextual factors that impact youths' experiences of empowerment were not explored. Within the youth empowerment literature, there is no empirical research on this type of potential negative effect. As a result, future research examining negative as well as positive impacts of contextual factors on psychological empowerment, particularly within restricted contexts, is encouraged.

A second potentially negative effect of empowerment exists within a specific subset of the incarcerated population, those youth with symptoms of psychopathology. Youth with symptoms of psychopathology might experience empowerment differently than youth without these characteristics, particularly the intrapersonal component of psychological empowerment. For example, youth expressing psychopathic characteristics might feel a sense of efficacy, competence, or other aspects of Intrapersonal PE that are unrelated to programming that seek to foster these qualities. Instead, beliefs related to the self might be inherent in youth with psychopathic traits. In addition to Intrapersonal PE, outcomes of psychological empowerment might be completely different for youth with psychopathic tendencies compared to youth that do 
not experience these symptoms. Outcomes of psychological empowerment for the youth who experience symptoms of psychopathology might have more potential to be negative. Examples of negative effects of psychological empowerment for these youth could include abusing one's sense of power by bullying or acting aggressively toward others. In the current study, psychological empowerment was not distinguished between youth who do and do not experience symptoms of psychopathology. Beyond this limitation, measuring psychopathology in youth is a controversial issue because adolescents are in a developmentally unique phase, which includes ego-centric behavior (Frick, 2002; Seagrave \& Grisso, 2002; Steinberg, 2002). Aspects of self-absorbed behaviors associated with adolescence may be characterized as psychopathic on measures of this construct. In assessing psychopathology in youth, it is possible to characterize normative behavior during this developmental phase as inaccurately reflecting psychopathology (Frick, 2002; Seagrave \& Grisso, 2002). A more thorough study of psychological empowerment's range of effects, particularly on youth that cannot enact their sense of empowerment and those that may express characteristics of psychopathology, would provide an additional foothold to establish predictive validity.

\section{Limitations Related to Predictions of Behavioral Success}

Other limitations to the current study, including the lack of establishment of causality, the lack of tracking major life events during the course of the study, the lack of randomization, lack of validity of the dependent variables, confounding of rater-bias and unit-level impacts, and missing staff data, impacted the second set of hypotheses. First, data on the independent (i.e., psychological empowerment in the three settings) and dependent (i.e., behavioral success in five domains) variables was collected cross- 
sectionally. Data collection on the dependent variables took place up to one month following the collection of the independent variables; however, some Treatment Managers or Unit Coordinators provided data for youth on the same day as data collection on the youth-reported independent variables. Furthermore, causality could not be inferred because temporal precedence was not established.

Variability in the time between data collection on the independent and dependent variables also weakened the design by allowing for potential significant events (e.g., fight, solitary confinement, transfer from one unit to the next) to change Treatment Managers' or Unit Coordinators' ratings of youth on the dependent variables. It was originally intended that all Treatment Managers or Unit Coordinators would rate youth competencies on the same day that youth provided data on psychological empowerment. This would have prevented untracked incidences from influencing the measurement of the dependent variable. Despite this planned design, the complexity and demands of their jobs prohibited some of the Treatment Managers or Unit Coordinators from completing the staff survey on the same day that data on the independent variable was collected. Within this study, it was not possible to track each participant's behavior during the time lag in order to ferret out the influence of significant negative events, such as staff-youth fights or placement into solitary confinement. It was possible, however, to track youth that transferred units during this timeframe. Using this information, cases were excluded if staff rated youth competencies for youth that they did not supervise in the living unit on the day of data collection for measures of the independent variable.

Another limitation threatening the internal validity of the current study was the lack of randomization of the sample population. The target population in the present 
research was all incarcerated young men in OYA correctional and re-entry facilities. In this case, the sampling frame nearly reflected the target population, where most of the incarcerated male youth in OYA correctional and re-entry facilities had the opportunity to participate in the study. Although the sample included a large percentage of all male youth residing within OYA facilities, the sample did not include all young men in OYA. Instead of random selection, youth participants included those present and interested in completing the survey at the time of data collection. As a result, the target population was undercovered, introducing coverage error to the sampling design (Groves, Fowler, Couper, Lepkowski, Singer, \& Tourangeua, 2009).

Validity of the dependent variables was also threatened because the dependent measures utilized within this dissertation were not psychometrically validated through previous empirical research. Construct validity and internal consistency of the dependent variables were threatened. Although they were not psychometrically examined, the measures of behavioral success were chosen from existing competencies associated with selected domains within OYA. OYA staff commonly rate these competencies, thus the measures of behavioral success included in the current study were context specific with high face validity. Additionally, OYA staff members were familiar with the rating system chosen for the measures of behavioral success as it was the same rating method that they use for regularly assessing OYA youth.

The validity of these results was also likely impacted by the fact that Treatment Manager or Unit Coordinator rater-bias was perfectly confounded with unit-level factors. In the mixed effects modeling, the living unit served as the grouping variable. The problem with this was that the staff that provided ratings of behavioral success in each 
unit only oversaw and reported on youth within a single unit. Thus, potential rater-bias and unit-level factors were confounded. While ICCs indicated that the living unit explained a great deal of variability in behavioral success within the five OYA domains, it is likely that rater-bias also explained much of this variability. When comparing unit level ICCs to facility-level ICCs, this possibility becomes obvious. Facility level ICCs, which were not confounded by rater-bias, indicated that facilities explain approximately $50 \%$ less variability in behavioral success than living units. It is possible that facilitylevel differences explain less variability in these scores than unit-level differences, but, in this case, it is impossible to ferret out unit-level differences from rater-bias. Due to this limitation, variability in behavioral success within the five OYA domains explained by unit-level differences was artificially inflated in these analyses.

Staff data was also problematic in that there was a large amount of missing data at one facility. Within one of the largest correctional facilities, $93 \%$ of staff-reported data on the youth was missing. This data was missing despite ample opportunity to respond and several reminders to complete the surveys. If OYA is interested in matching youth and staff data in the future, it will be critical to ensure a better response rate among staff, particularly within this facility.

Strengths, Implications, and Future Research Directions

While this study encountered several limitations, aspects of its design were intended to strengthen the researcher's ability to determine differences in reporting and outcomes of psychological empowerment within different correctional settings. Although the results of this investigation did not support the research hypotheses, findings can be used to make specific suggestions for future research that will help to 
reconceptualize and reoperationalize components of psychological empowerment. Recommendations can also be made to help identify other concepts related to psychological empowerment that should be measured simultaneously. These strengths and future research directions as well as implications of this line of research will be discussed next.

One strength of the current research was that psychological empowerment was investigated in different contexts. More specifically, this study recognized that psychological empowerment is context-dependent. Contextual differences were taken into account by examining the endorsement of psychological empowerment in three settings in the correctional and re-entry facilities (i.e., treatment group, school or vocational activities, living unit). By allowing scores to vary between settings, the experience of psychological empowerment between settings was not conflated.

Upon reflection of several limitations related to measuring psychological empowerment (e.g., frustration with repetitive questions), the researcher has made several recommendations. To improve upon this strength, it is suggested that alternative instructional formats are explored, making participants more cognizant of the setting in which their responses refer. In addition to changing the instructions, psychological empowerment scales in each setting should not be placed consecutively within the survey. Instead, other scales, such as LOC, HPES, and HCCQ, should be placed strategically between psychological empowerment scales in different settings so that participants are not acutely aware that the items are repetitive.

Despite the attempt to strengthen the design by measuring psychological empowerment in different settings, the operationalization of psychological empowerment 
was flawed. The three-factor model was rejected, and the Interactional and Behavioral PE single-factor models were not supported. The Intrapersonal PE sub-scale was the only sub-scale supported by factor analyses, but there was still a large amount of measurement error involved in the assessment of Intrapersonal PE. Due to insufficient operationalization, psychological empowerment, particularly the Interactional and Behavioral components of psychological empowerment, should be reconceptualized and reoperationalized.

Based on results of the present study, the researcher has made several specific suggestions for reoperationalization. First, the Intrapersonal PE sub-scale should incorporate more items, particularly related to perceived control and motivation to control. The concept of perceived competence has also been suggested as an underlying construct of intrapersonal psychological empowerment (Zimmerman \& Warschausky, 1998). Perceived competence was not included as a domain of Intrapersonal PE in this study, but it might be worth the effort to develop items assessing perceived competence in order to expand the domain breadth of this component of psychological empowerment, increasing its content validity.

Second, the Behavioral PE factor structure should be reassessed in each setting. Similar to the exploratory process undertaken to examine the factor structure of Behavioral PE in the living unit, a split-half EFA/CFA might be more useful to identifying an acceptable model than fitting the model of Behavioral PE in the living unit to data in the other two settings. If an appropriate factor structure cannot be determined, additional items should be developed to assess Behavioral PE. In creating additional items to measure Behavioral PE, actual behaviors or actions enacted by youth that are 
specific to the context in which psychological empowerment is experienced should be measured. It is possible that response formats alternative to a Likert scale response format, such as the one used in the present research, might be more appropriate for assessing these behaviors.

Third, the researcher firmly believes that the conceptualization and operationalization of Interactional PE component should be reviewed. The items developed were found to be confusing and did not relate well with one another. Similar to Behavioral PE, Interactional PE is also highly context dependent. Less generic, more specific items might need to be developed in order to adequately assess this component of psychological empowerment.

Fourth, there might be utility in including expert opinion within two distinct fields. Consultation by expert researchers that have experience quantifying psychological empowerment should be sought. Feedback on domain breadth and item wording would help to improve content and face validities of the scale. Another important source of feedback is from potential participants (i.e., incarcerated youth) and OYA staff. Their input on the scale should also be integrated into the measure development process. It is anticipated that doing so would better ensure ecological validity of the scale.

The researcher also strongly advocates for the integration of qualitative methodology into the design. Through this methodology, investigators would also be better able to explore the ways in which empowerment is experienced in each of the various critical settings in which the youth live and function (e.g., treatment groups, living unit). Using an iterative, mixed-methods approach would allow researchers to triangulate the conceptualization and operationalization of psychological empowerment, 
further validating the construct. Beyond the confirmation of psychological empowerment's conceptualization, qualitative work would provide researchers with the tools to explore the negative effects of psychological empowerment and address questions related to the "glass ceiling." Qualitative methods are uniquely suited to the study of negative cases of empowerment (e.g., examples of youth that do not experience empowerment) because they often allow researchers to probe deep into an aspect of the topic that might not surface otherwise. An additional benefit to using mixedmethodology would also allow for the perspectives and voices of incarcerated youth to be more fully incorporated into the research process. Familiarity with youths' perspectives on psychological empowerment could allow researchers to better integrate language familiar to and often utilized by youth into the scale items, improving the operationalization of the construct.

Considering the limited piloting process in the current study, the researcher suggests that one method for integrating qualitative researcher into the development of this measure would be to utilize the time with the Youth Advisory Committee (YAC) differently. Feedback from the YAC was a strength in the present study. Upon reflection, however, time spent with the youth could have been maximized by treating the session more as a pilot than as a forum for general questions. A pilot study could have been conducted using aspects of cognitive interviewing, where participants engage in "retrospective think-alouds," describing their thought process as they arrive at their response (Groves, Fowler, Couper, Lepkowski, Singer, \& Tourangeau, 2009). This type of pilot study could further inform item wording and scale formatting. Other information that could be collected during "think-alouds" could pertain to whether or not youth have 
power-oriented goals within different OYA settings and how these goals relate to the various survey items. Rather than designing the meeting around informal conversation about the survey as a whole, this pilot technique would provide the more structure to engage youth in a self-guided discussion about each item and how it potentially relates to aspects of empowerment.

Through the triangulation of findings within the present research as well as findings from qualitative research such as the pilot study mentioned above, it is advised that the conceptualization of all three components of psychological empowerment be reconsidered. It has been assumed that empowerment cannot occur within settings that are considered to be rigid and that limit one's autonomy or self-determination (Lightfoot, 1986). In this vein, characteristics associated with empowering settings (e.g., strengthsbased, collective, collaborative, diversity in roles, supportive; Maton, 2008; Maton \& Salem, 1995), do not typically coincide with common depictions of youth correctional settings. Oft cited descriptions of the juvenile justice system include "rigid" and "punitive," and the culture has been accused of exclusively focusing on negative aspects of the self (Butts \& Mears, 2001; Corcoran, 1997; Hunter et al., 2004; Marshall et al., 2005). This misalignment does not necessarily negate the notion that empowerment, as both a state and as a process, can occur within correctional settings. While there is little research explicitly aimed at studying empowerment in rigid and restrictive settings, there is evidence that suggests that empowerment can occur within settings that do not typically match Maton's (2008) description. For instance, nurses working in controlled environments have reported access to empowerment structures (Almost \& Laschinger, 2002; Laschinger, Sabiston, Kutszcher, 1997; Patrick \& Laschinger, 2006), and 
empowerment models have been posed for students facing institutional issues related to minority status and other environment-student need misfit (Bemak et al., 2005; Cleary \& Zimmerman, 2004). Although empowerment in these types of settings does not come without difficulties, particularly related to inequality that trickles down from high levels of an institution (Gruber \& Trickett, 1987), these examples demonstrate that empowerment is possible, even when the environment is hierarchical and constrained in nature.

While it is still possible the empowerment occurs in this bounded setting, the definition of psychological empowerment might ultimately look different in a youth correctional context than the one originally put forth by Zimmerman (1995) and colleagues (1998). Youth might have limited capacity to make social or structural changes to their environment. For example, incarcerated youth will always have limited contact with the surrounding community. Despite these restrictions, youth might be able to make personally meaningful changes to their educational, vocational, or treatment goals as well as affect the culture within their living units. While these smaller goals might not be directly related to the power differential between them and the institution, for example, they may still relate to personally meaningful changes that can lead to structural changes at higher levels. The case of a young man at one of the three transition facilities can be used as a primary example. A youth preparing to transition back into the community began thinking about what kind of career he would like to pursue once released from OYA custody. He decided that his skills and interests were best suited to waste water maintenance. Through his collaboration with staff, the Vocational Education Services for Older Youth, and community members, the youth was able to help establish 
a quasi-internship for youth with certification in water maintenance to help prepare for the future (C. McClellan, personal communication, November 17, 2011). It is possible to see that elements of empowerment are present within this example. The youth in this example defined a meaningful goal, which was restricted by a structured environment that did not provide the resources for him to achieve his goal. By collaborating with others and utilizing resources that were available to him, he was able to overcome these limitations and helped to put in place a program that would benefit other youth in the future. Additional work to better understand how empowerment-related (i.e., poweroriented) goals and, more broadly, psychological empowerment are defined from the youths' perspectives would help reconcile differences between Zimmerman's operationalization of psychological empowerment and the present study's findings.

As a result of several limitations related to contextual factors, the researcher suggests that other aspects of empowerment should be explored. As previously discussed, youth should be asked about their power-oriented goals in each setting. By doing so, researchers would have a better idea about: (1) the ways in which youth perceive power differentials in their environment and (2) which power-oriented goals are and are not being met through an empowerment process in different contexts. By measuring power-oriented goals, investigators could also ensure that youth have identified an aspect of their lives that is impacted by lack of power and are working toward achieving some goal related to this realization rather than assuming this to be the case.

In order to place these goals into context, the researcher believes that it would be useful to incorporate OYA staff input on goals. OYA staff have a realistic view of the 
context; thus they are in a unique position to provide input on the possibility for youth to achieve their goals within each setting. Staff input regarding the plausibility of goal achievement within the correctional setting could also lead to studies investigating the effects of perceived psychological empowerment when the state of the environment does not allow for change from the bottom up.

Second, antecedents and correlates of psychological empowerment should be examined. The validity of the scales used to establish discriminant validity in this study should be confirmed, and the relationships between these variables (i.e., prison environment, locus of control, and treatment autonomy) and psychological empowerment should be more thoroughly investigated. In doing so, construct validity of psychological empowerment would be strengthened. Additionally, psychological empowerment would be better understood if the ways in which contextual level variables (e.g., staff ratings of empowerment, characteristics of the physical environment) impact this construct in different settings.

This research has demonstrated that psychological empowerment is complex and difficult to measure. It is a broad construct, encompassing many domains. As has been discussed, there are three primary components (i.e., intrapersonal, interactional, behavioral) of psychological empowerment. In this operationalization, each of the three components consists of three domains, some of which are fairly broad constructs in and of themselves (e.g., self-efficacy). The inclusion of nine total constructs (i.e., domains) within the entire scale might have decreased the likelihood of finding a well-fitting threefactor model. Even within individual components, this issue was observed. The three domains within Behavioral PE, for example, were questioned as measuring more than 
behavioral markers of psychological empowerment. In order to cover the entire spectrum of content within psychological empowerment, many facets must be included in its assessment. Modest associations between these concepts makes it difficult to assess psychological empowerment overall. This is, perhaps, one of the reasons that a similar three-factor model of psychological empowerment was not found within the research literature.

Second, this study recognized that psychological empowerment is shaped by individual and contextual factors. As a result, empowerment could be expressed in a variety of ways, yet item wording did not reflect this notion. The items within the scales were worded in a way that assumed that empowerment would be expressed consistently across the settings. Take for instance, the item under the involvement domain of Behavioral PE, which read "I actively participate in activities, even if I don't have to." It was assumed that empowerment would be demonstrated as voluntary involvement. While voluntary involvement might be characteristic of some empowered youth, other youth that also feel empowered might decide to use their time differently in order to advance their goals. This example helps to illuminate the problem with assuming directionality of the items in this operationalization of psychological empowerment.

While it might be difficult to measure, it is worth the sustained effort to establish a working scale of psychological empowerment. In addition to the reconceptualization and reoperationalization of psychological empowerment through mixed methods and the inclusion of expert opinions, the researcher has several other suggestions on how to revise the scale that would help account for the construct's broad scope and directionality of the items. Other scales on psychological empowerment for youth are geared toward a 
specific problem or issue. For example, the scale developed by Holden and colleagues $(2004 ; 2005)$ centered on a tobacco prevention program for youth. Akey and her colleagues' (2000) measure of psychological empowerment was constructed for parents of children with a disability. These scales have a single focus or goal for empowerment programming. In contrast, incarcerated youth work on any number of criminal behaviors and psychosocial skills in several settings within correctional facilities. In order to narrow the focus of the current scale and match the scope of previously established measures, items within each OYA setting could benefit from the specification of a single issue within that context (e.g., achieving high school diploma or equivalency in school, identifying and working toward a career). Although the development of items related to a certain issue within each context would narrow the scope of the construct, this explicitness might limit the number of youth able to respond to the issue as not all youth work toward the same goals. An alternative to this specificity is to situate broad items within youths' power-oriented goals.

By instructing youth to identify and articulate one power-oriented goal within each context, youth might be more attuned to psychological empowerment items in three different scales, even if item content is repetitive. The items would be inherently nested within a given context that is self- rather than researcher- or staff-defined. Situating items within power-oriented goals would continue to allow youth scores to vary by individual but could also provide clarity to issues related to the directionality of items. In the example item above, "I actively participate in activities, even if I don't have to," psychological empowerment would best be expressed by voluntary participation in activities associated with a youth's self-defined goal. Another benefit to instructing 
youth to respond to items while thinking about their power-oriented goals is that investigators could be more certain that youth actually have some kind of goal or issue that they are interested in changing within each setting. For these reasons, the researcher advocates for revisions to the instructions and to the introduction of each scale in a way that would position youth to respond to items related only to their own power-oriented goal in each OYA setting.

While an acceptable scale of psychological empowerment has yet to be identified, some foundations for its measurement have been laid. The basis for a sub-scale measuring Intrapersonal PE in three correctional settings was established in the present study. This sub-scale could be enhanced by the addition of items that concisely tap into motivation to control and perceived control. Although sub-scales measuring Interactional and Behavioral PE were not established, further exploratory analyses of Behavioral PE in the treatment and school or vocational settings might produce justifiable sub-scales for these settings. Through this research, it has also been established that Interactional PE might look different than its original definition developed within community-based settings and that Behavioral PE should most likely be conceptualized within very specific settings or, in the least, nested under a power-oriented goal. While findings regarding the factor structure did not support the research hypotheses, continued work on psychological empowerment is further warranted.

Research that continues to explore the definition and measurement of psychological empowerment in a youth correctional context has the potential to impact general research in this area as well as future OYA work and evaluation directions. First, research along these lines would continue to extend literature on psychological 
empowerment into a new context. It would also allow researchers to respond to and advance the call to explore alternative, positive, rehabilitative models of treatment and intervention for incarcerated youth made by progressive minds in the field.

In addition to the potential to inform the understanding of psychological empowerment in a juvenile correctional context, the establishment of a measure of psychological empowerment within one or more youth correctional settings has the potential to enable future research and evaluation work at OYA and other similar institutions across the country. Equipped with a tool to measure psychological empowerment, researchers at OYA could better explore the antecedents, correlates, and outcomes of psychological empowerment. Specifically, researchers at OYA could identify aspects of their programming and the environmental context more generally that facilitate the experience of empowerment. OYA would be better able to evaluate the impact of programming that is implicitly and/or explicitly guided by empowerment-based principles. They could also identify and assess empowerment-related goals that are thwarted by contextual constraints. In some cases, this type of evaluative feedback could lead to positive program modifications. These revisions could extend opportunities for youth to set appropriate power-oriented goals and advance their growth in areas related to attaining successful treatment outcomes.

More resources will be required to establish a solid measure of psychological empowerment for use within a youth correctional context, as the process for doing so is both iterative and reflective. However, it is worth an extended effort to develop such a measure. As demonstrated in the Chapter V, empowerment can be developed through several avenues within OYA programming. In addition to programming, it has been 
implicitly and explicitly defined as a goal for youth participating in specific programs by several treatment managers. In order to evaluate OYA's ability to engage youth in the empowerment process and evoke a state of psychological empowerment, a method to quantify this complex construct is required.

In addition to the need for this measure, the present research has begun to examine beneficial correlates of empowerment related efforts. Using an imperfect measure of Intrapersonal PE, which was impacted heavily by measurement error, Intrapersonal PE in treatment and school or vocational activities significantly predicted behavioral success. Due to unsupported factor structures of Interactional and Behavioral PE, psychological empowerment's actual ability to predict behavioral success cannot be determined. It can only be assessed with validated, reliable measures of its sub-scales. Furthermore, research advancing the conceptualization and operationalization of the three components of psychological empowerment will allow researchers within OYA and the juvenile justice field to better study some of the positive, strengths-based aspects of programming that practitioners have called for. For example, as previously mentioned, a tool to measure psychological empowerment would allow OYA researchers to evaluate the outcomes of programming aimed at positively impacting self-efficacy, perceived control, locus of control, and other empowerment-related concepts. In addition to outcomes, contextual factors that facilitate or inhibit the experience of psychological empowerment and its components could be assessed more easily. Although correctional facilities are, at this point in time, characterized as rigid and deficits-based, continued research in this field has to the potential to fuel the movement for progressive, innovative, strengths-based programming and environments for incarcerated youth. 
Table 1

\section{Selected Items and Abbreviations}

\begin{tabular}{|c|c|c|c|c|}
\hline \multicolumn{5}{|r|}{ Living Unit } \\
\hline Component & Domain & Item ID & Order & Item \\
\hline Intrapersonal & Self-efficacy & $\mathrm{SE} 1 *$ & 1 & If something looks too complicated, I will not even bother to try it. \\
\hline Intrapersonal & $\begin{array}{l}\text { Self-efficacy } \\
\text { Motivation to }\end{array}$ & SE2 & 10 & I am confident that I can work effectively on many different tasks. \\
\hline Intrapersonal & $\begin{array}{l}\text { Control } \\
\text { Motivation to }\end{array}$ & $\mathrm{MC1}$ & 2 & In general, I would prefer to be a leader rather than a follower. \\
\hline Intrapersonal & $\begin{array}{l}\text { Control } \\
\text { Perceived }\end{array}$ & $\mathrm{MC} 2 *$ & 11 & When I work on group projects, I prefer to "take a back seat." \\
\hline Intrapersonal & $\begin{array}{l}\text { Control } \\
\text { Perceived }\end{array}$ & PC1 & 3 & $\begin{array}{l}\text { I can influence decisions that are made. } \\
\text { There is little I can do to change many of the things that are important to }\end{array}$ \\
\hline Intrapersonal & Control & $\mathrm{PC} 2 *$ & 12 & $\begin{array}{l}\text { me. } \\
\text {. }\end{array}$ \\
\hline Interactional & $\begin{array}{l}\text { Awareness of } \\
\text { Resources }\end{array}$ & AR1 & 13 & $\begin{array}{l}\text { I know where to go to get information about starting a new activity like a } \\
\text { basketball tournament. }\end{array}$ \\
\hline Interactional & $\begin{array}{l}\text { Awareness of } \\
\text { Resources } \\
\text { Critical }\end{array}$ & $\mathrm{AR} 2 *$ & 4 & $\begin{array}{l}\text { I am unsure about where to go if I have a major problem with an OYA } \\
\text { staff member, like he or she makes fun of me and it hurts my feelings. }\end{array}$ \\
\hline Interactional & $\begin{array}{l}\text { Awareness } \\
\text { Critical }\end{array}$ & CA1 & 5 & The beliefs of other youth at OYA make it difficult to do what is right. \\
\hline Interactional & $\begin{array}{l}\text { Awareness } \\
\text { Problem }\end{array}$ & $\mathrm{CA} 2 *$ & 14 & $\begin{array}{l}\text { OYA staff have little influence on my behavior. } \\
\text { When making a decision, I weigh the consequences of each choice and }\end{array}$ \\
\hline Interactional & $\begin{array}{l}\text { Solving } \\
\text { Problem }\end{array}$ & PS1 & 6 & $\begin{array}{l}\text { compare them against each other. } \\
\text { When confronted with a problem, I tend to do the first thing that I can }\end{array}$ \\
\hline Interactional & Solving & $\mathrm{PS} 2 *$ & 15 & think of to solve it. \\
\hline Behavioral & Advocacy & A1 & 16 & $\begin{array}{l}\text { I speak up about issues that are important to me. } \\
\text { Let things } g_{0} \text { rather than to speak un about them even if they are }\end{array}$ \\
\hline Behavioral & Advocacy & $\mathrm{A} 2 *$ & 7 & important to me. \\
\hline Behavioral & Involvement & I1 & 8 & I actively participate in activities, even if I don't have to. \\
\hline Behavioral & Involvement & $\mathrm{I} 2 *$ & 17 & $\begin{array}{l}\text { If given the choice, I prefer to do other things rather than participate in } \\
\text { activities. }\end{array}$ \\
\hline Behavioral & Coping & $\mathrm{C} 1$ & 18 & There is at least one other youth I can go to for support. \\
\hline Behavioral & Coping & $\mathrm{C} 2 *$ & 9 & I only have myself to rely on for support. \\
\hline
\end{tabular}




\begin{tabular}{|c|c|c|c|c|}
\hline Component & Domain & Item ID & Order & Item \\
\hline Intrapersonal & Self-efficacy & $\mathrm{SE} 1 *$ & 9 & If something looks too complicated, I will not even bother to try it. \\
\hline Intrapersonal & $\begin{array}{l}\text { Self-efficacy } \\
\text { Motivation to }\end{array}$ & SE2 & 1 & I am confident that I can work effectively on many different tasks. \\
\hline Intrapersonal & $\begin{array}{l}\text { Control } \\
\text { Motivation to }\end{array}$ & $\mathrm{MC} 1$ & 10 & In general, I would prefer to be a leader rather than a follower. \\
\hline Intrapersonal & $\begin{array}{l}\text { Control } \\
\text { Perceived }\end{array}$ & $\mathrm{MC} 2 *$ & 2 & When I work on group projects, I prefer to "take a back seat." \\
\hline Intrapersonal & $\begin{array}{l}\text { Control } \\
\text { Perceived }\end{array}$ & PC1 & 12 & $\begin{array}{l}\text { I can influence decisions that are made. } \\
\text { There is little I can do to change many of the things that are important to }\end{array}$ \\
\hline Intrapersonal & $\begin{array}{l}\text { Control } \\
\text { Awareness of }\end{array}$ & $\mathrm{PC} 2 *$ & 3 & $\begin{array}{l}\text { me. } \\
\text { I know where to go to get information about my progress on my }\end{array}$ \\
\hline Interactional & Resources & AR1 & 4 & treatment goals. \\
\hline Interactional & $\begin{array}{l}\text { Awareness of } \\
\text { Resources } \\
\text { Critical }\end{array}$ & $\mathrm{AR} 2 *$ & 13 & $\begin{array}{l}\text { I am unsure about where to go if I have a major problem with an OYA } \\
\text { staff member, like I disagree with something she or he asked me to work } \\
\text { on. }\end{array}$ \\
\hline Interactional & $\begin{array}{l}\text { Awareness } \\
\text { Critical }\end{array}$ & CA1 & 14 & The beliefs of other youth at OYA make it difficult to do what is right. \\
\hline Interactional & $\begin{array}{l}\text { Awareness } \\
\text { Problem }\end{array}$ & $\mathrm{CA} 2 *$ & 5 & $\begin{array}{l}\text { OYA staff have little influence on my behavior. } \\
\text { When making a decision, I weigh the consequences of each choice and }\end{array}$ \\
\hline Interactional & $\begin{array}{l}\text { Solving } \\
\text { Problem }\end{array}$ & PS1 & 15 & $\begin{array}{l}\text { compare them against each other. } \\
\text { When confronted with a problem, I tend to do the first thing that I can }\end{array}$ \\
\hline Interactional & Solving & PS2* & 6 & think of to solve it. \\
\hline Behavioral & Advocacy & A1 & 7 & $\begin{array}{l}\text { I speak up about issues that are important to me. } \\
\text { I let things go rather than to speak up about them, even if they are }\end{array}$ \\
\hline Behavioral & Advocacy & $\mathrm{A} 2 *$ & 16 & important to me. \\
\hline Behavioral & Involvement & I1 & 17 & $\begin{array}{l}\text { I actively participate in activities, even if I don't have to. } \\
\text { If given the choice, I prefer to do other things rather than participate in }\end{array}$ \\
\hline Behavioral & Involvement & $\mathrm{I} 2 *$ & 8 & activities. \\
\hline Behavioral & Coping & $\mathrm{C} 1$ & 18 & There is at least one other youth I can go to for support. \\
\hline Behavioral & Coping & $\mathrm{C} 2 *$ & 11 & I only have myself to rely on for support. \\
\hline
\end{tabular}


School or Vocational Activities

\begin{tabular}{|c|c|c|c|c|}
\hline Component & Domain & Item ID & Order & Item \\
\hline Intrapersonal & Self-efficacy & $\mathrm{SE} 1 *$ & 5 & If something looks too complicated, I will not even bother to try it. \\
\hline Intrapersonal & $\begin{array}{l}\text { Self-efficacy } \\
\text { Motivation to }\end{array}$ & SE2 & 13 & I am confident that I can work effectively on many different tasks. \\
\hline Intrapersonal & $\begin{array}{l}\text { Control } \\
\text { Motivation to }\end{array}$ & $\mathrm{MC} 1$ & 14 & In general, I would prefer to be a leader rather than a follower. \\
\hline Intrapersonal & $\begin{array}{l}\text { Control } \\
\text { Perceived }\end{array}$ & $\mathrm{MC} 2 *$ & 4 & When I work on group projects, I prefer to "take a back seat." \\
\hline Intrapersonal & $\begin{array}{l}\text { Control } \\
\text { Perceived }\end{array}$ & PC1 & 3 & $\begin{array}{l}\text { I can influence decisions that are made. } \\
\text { There is little I can do to change many of the things that are important to }\end{array}$ \\
\hline Intrapersonal & $\begin{array}{l}\text { Control } \\
\text { Awareness of }\end{array}$ & $\mathrm{PC} 2 *$ & 12 & $\begin{array}{l}\text { me. } \\
\text { I know where to go to get information about taking classes outside of }\end{array}$ \\
\hline Interactional & $\begin{array}{l}\text { Resources } \\
\text { Awareness of }\end{array}$ & AR1 & 11 & $\begin{array}{l}\text { OYA. } \\
\text { I am unsure about where to go if I have a major problem with an OYA }\end{array}$ \\
\hline Interactional & $\begin{array}{l}\text { Resources } \\
\text { Critical }\end{array}$ & $\mathrm{AR} 2 *$ & 2 & staff member, like he or she says mean or rude things about my work. \\
\hline Interactional & $\begin{array}{l}\text { Awareness } \\
\text { Critical }\end{array}$ & CA1 & 1 & The beliefs of other youth at OYA make it difficult to do what is right. \\
\hline Interactional & $\begin{array}{l}\text { Awareness } \\
\text { Problem }\end{array}$ & $\mathrm{CA} 2 *$ & 10 & $\begin{array}{l}\text { OYA staff have little influence on my behavior. } \\
\text { When making a decision, I weigh the consequences of each choice and }\end{array}$ \\
\hline Interactional & $\begin{array}{l}\text { Solving } \\
\text { Perceived }\end{array}$ & PS1 & 6 & $\begin{array}{l}\text { compare them against each other. } \\
\text { When confronted with a problem, I tend to do the first thing that I can }\end{array}$ \\
\hline Interactional & Control & $\mathrm{PS} 2 *$ & 15 & think of to solve it. \\
\hline Behavioral & Advocacy & A1 & 16 & $\begin{array}{l}\text { I speak up about issues that are important to me. } \\
\text { I let things go rather than to speak up about them, even if they are }\end{array}$ \\
\hline Behavioral & Advocacy & $\mathrm{A} 2 *$ & 7 & important to me. \\
\hline Behavioral & Involvement & I1 & 17 & $\begin{array}{l}\text { I actively participate in activities, even if I don't have to. } \\
\text { If given the choice, I prefer to do other things rather than participate in }\end{array}$ \\
\hline Behavioral & Involvement & $\mathrm{I} 2 *$ & 8 & activities. \\
\hline Behavioral & Coping & $\mathrm{C} 1$ & 9 & There is at least one other youth I can go to for support. \\
\hline Behavioral & Coping & $\mathrm{C} 2 *$ & 18 & I only have myself to rely on for support. \\
\hline
\end{tabular}

* Reverse coded. 


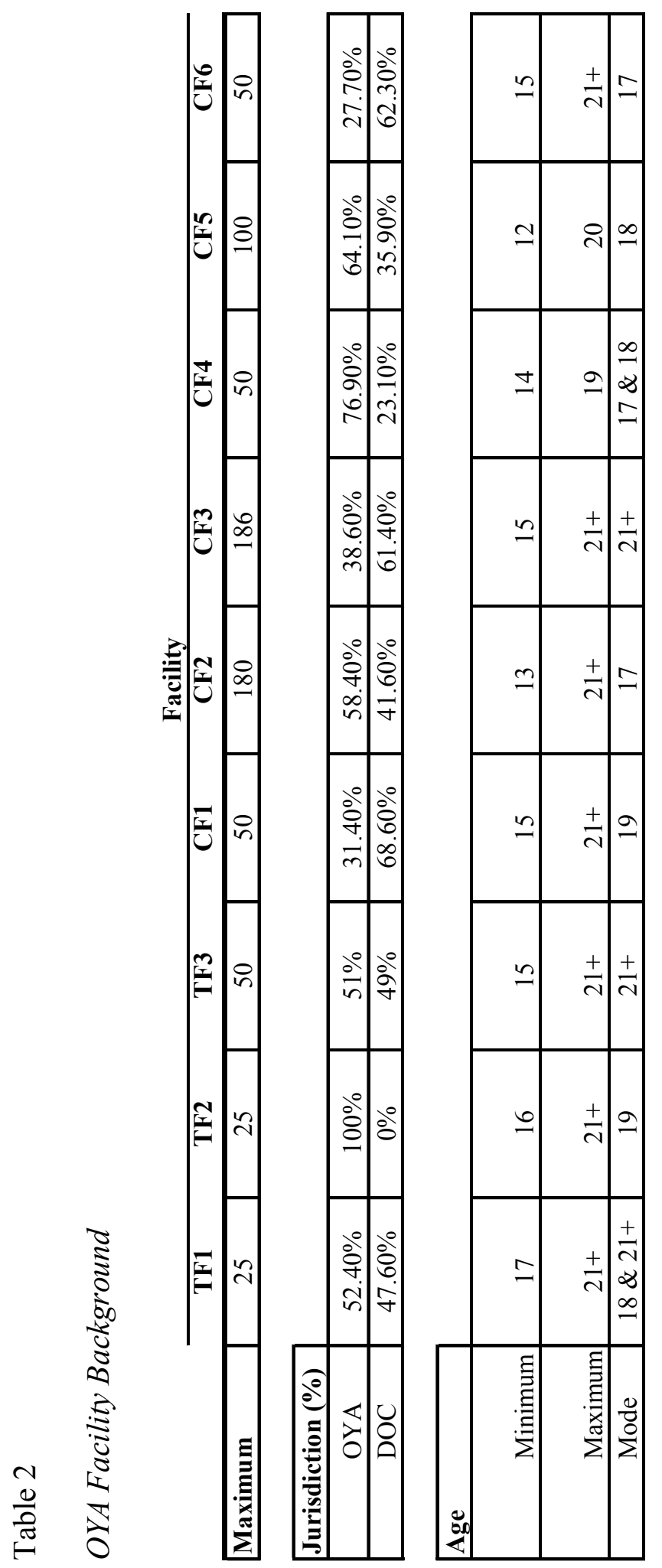




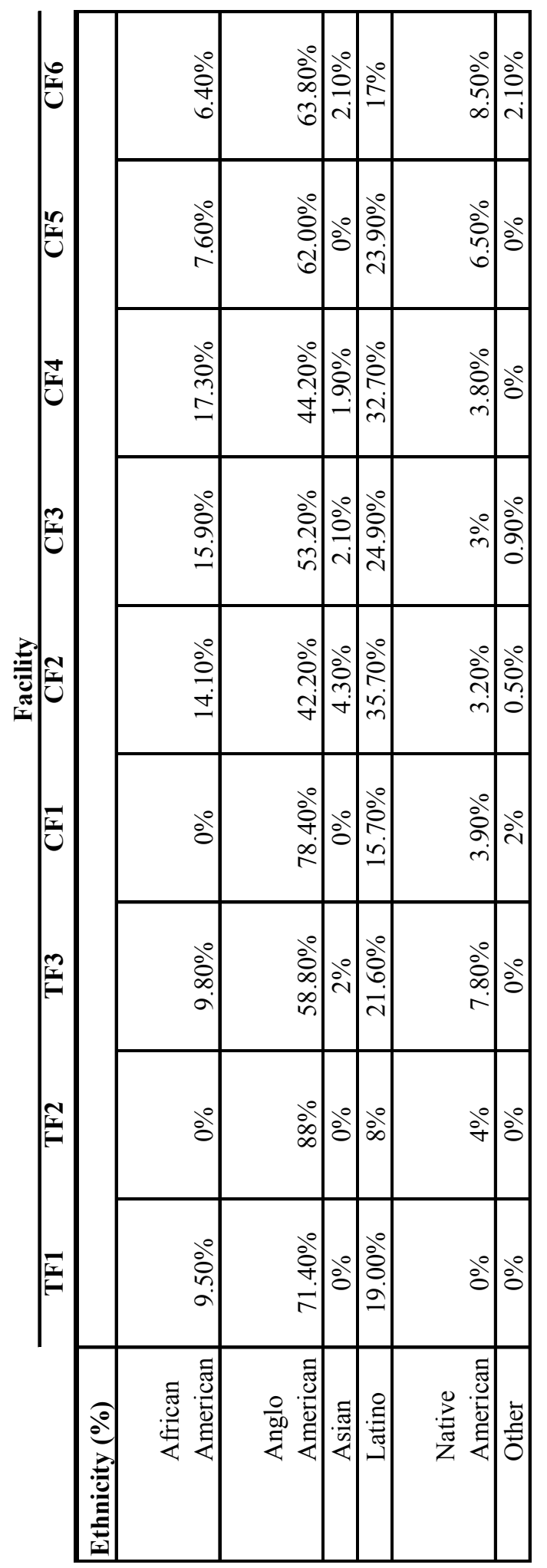




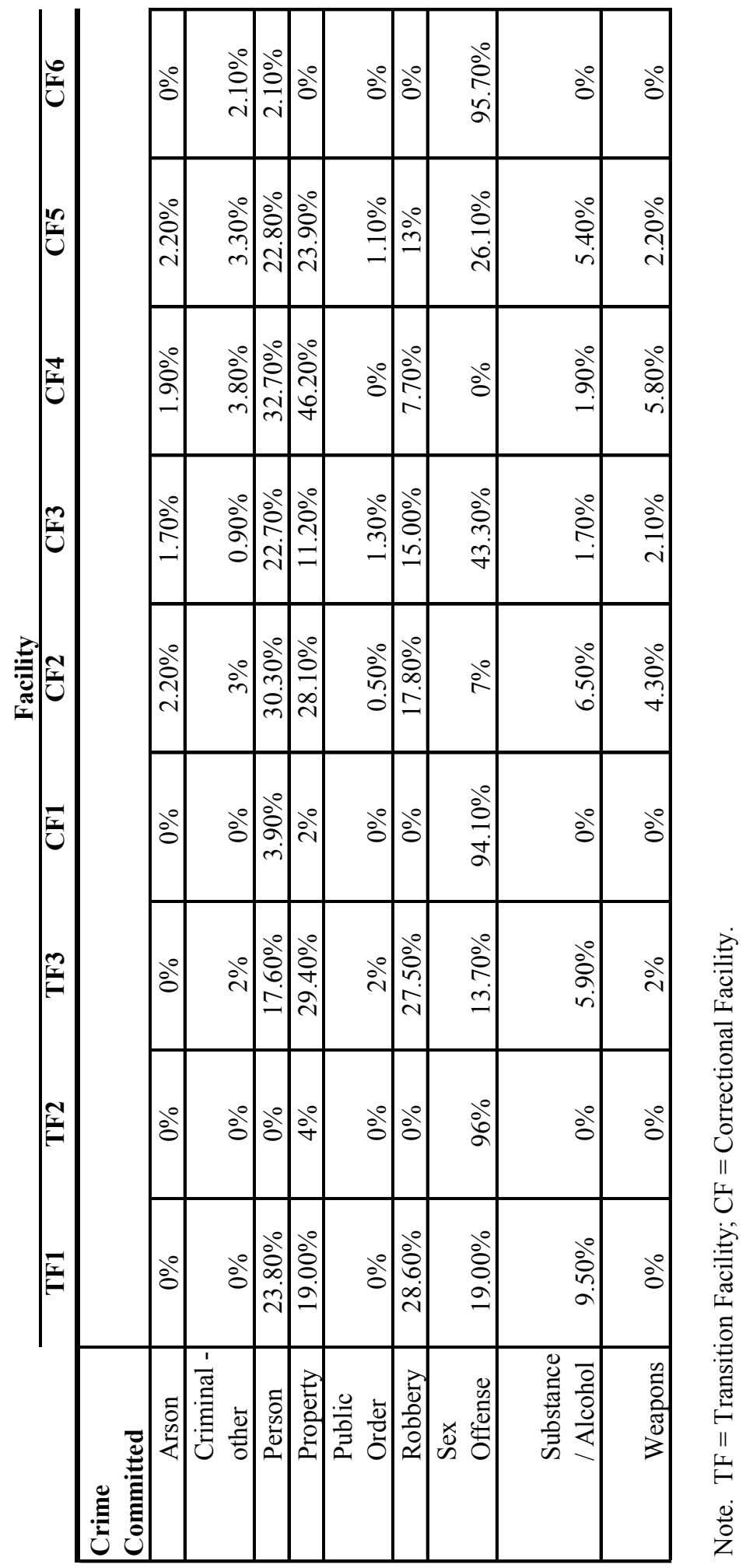


Table 3

Demographic Information for the Sample of Incarcerated Youth

\begin{tabular}{|c|c|c|c|c|c|}
\hline & \multicolumn{2}{|c|}{ Sample Population } & \multicolumn{2}{|c|}{ General Population* } \\
\hline & & $M(S D)$ & Median & $M(S D)$ & Median \\
\hline Age & & $18.43(2.43)$ & 18 & NA & 18 \\
\hline \multirow[t]{3}{*}{ Total Time Incarcerated } & & $2.20(1.94)$ & 2.00 & $2.08(1.88)$ & 1.42 \\
\hline & & \multicolumn{2}{|c|}{ Sample Population } & \multicolumn{2}{|c|}{ General Population* } \\
\hline & & $\mathrm{N}$ & Percent & $\mathrm{N}$ & Percent \\
\hline \multirow[t]{7}{*}{ Race/Ethnicity } & & 550 & & 697 & \\
\hline & African American & 58 & $10.5 \%$ & 80 & $11.5 \%$ \\
\hline & Anglo American & 302 & $54.9 \%$ & 367 & $52.7 \%$ \\
\hline & Asian & 12 & $2.2 \%$ & 14 & $2.0 \%$ \\
\hline & Latino & 148 & $26.9 \%$ & 200 & $28.7 \%$ \\
\hline & Native American & 28 & $5.1 \%$ & 32 & $4.6 \%$ \\
\hline & Other/Unknown & 2 & $0.4 \%$ & 3 & $0.4 \%$ \\
\hline \multirow{3}{*}{ Commitment Disposition } & & 550 & & 697 & \\
\hline & OYA & 280 & $50.9 \%$ & 344 & $49.4 \%$ \\
\hline & DOC & 270 & $49.1 \%$ & 353 & $50.7 \%$ \\
\hline \multirow[t]{16}{*}{ Offense Category } & & 550 & & 697 & \\
\hline & Arson & 7 & $1.3 \%$ & 10 & $1.4 \%$ \\
\hline & Assault & 91 & $16.5 \%$ & NA & \\
\hline & Burglary & 46 & $8.4 \%$ & NA & \\
\hline & Criminal Mischief & 11 & $2.0 \%$ & NA & \\
\hline & Criminal "Other" & 5 & $0.9 \%$ & 9 & $1.3 \%$ \\
\hline & Harassment & 1 & $0.2 \%$ & NA & \\
\hline & Homicide Related & 25 & $4.5 \%$ & NA & \\
\hline & Person "Other" & 13 & $2.4 \%$ & 171 & $24.5 \%$ \\
\hline & Public Order "Other" & 2 & $0.4 \%$ & 4 & $0.6 \%$ \\
\hline & Robbery & 70 & $12.7 \%$ & 97 & $13.9 \%$ \\
\hline & Sex Offense & 206 & $37.5 \%$ & 249 & $35.7 \%$ \\
\hline & Substance Abuse & 19 & $3.5 \%$ & 18 & $2.3 \%$ \\
\hline & Theft & 33 & $6.0 \%$ & NA & \\
\hline & Weapons & 18 & $3.3 \%$ & 28 & $4.0 \%$ \\
\hline & Criminal Trespassing & 3 & $0.5 \%$ & NA & \\
\hline
\end{tabular}

* General population data based on totals from November 1, 2012. 
Table 4

Facility Sub-sample Sizes

\begin{tabular}{lcc} 
& $\begin{array}{c}\text { OYA Annual } \\
\text { Survey } \\
\text { Participants } \\
\mathrm{n}(\%)\end{array}$ & $\begin{array}{c}\text { OYA Staff } \\
\text { Ratings of } \\
\text { Behavioral } \\
\text { Success } \\
\mathrm{n}(\%)^{*}\end{array}$ \\
\hline TF1 & $17(81 \%)$ & $15(71 \%)$ \\
TF2 & $21(84 \%)$ & $20(80 \%)$ \\
TF3 & $45(98 \%)$ & $42(91 \%)$ \\
CF1 & $47(94 \%)$ & $44(88 \%)$ \\
CF2 & $121(75 \%)$ & $100(62 \%)$ \\
CF3 & $111(59 \%)$ & $12(6 \%)$ \\
CF4 & $47(90 \%)$ & $45(87 \%)$ \\
CF5 & $94(93 \%)$ & $89(96 \%)$ \\
CF6 & $47(90 \%)$ & $22(42 \%)$
\end{tabular}

Note. 'TF' refers to transition facility. 'CF' refers to correctional facility. Percentages based off of total facility populations on November 1, 2012.

$*_{\mathrm{n}}$ and $\%$ are for ratings of youth, not for total number of responding staff. 


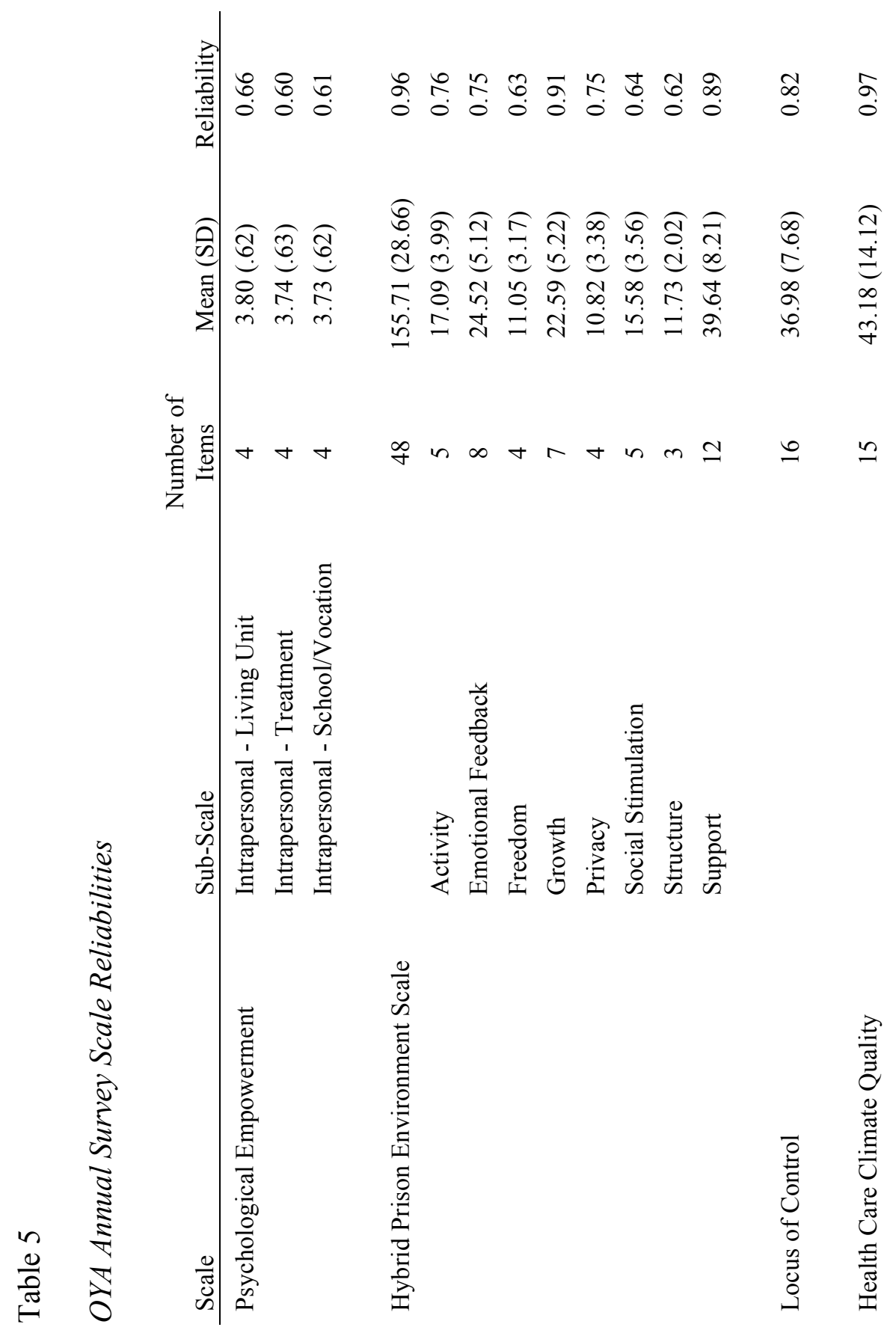




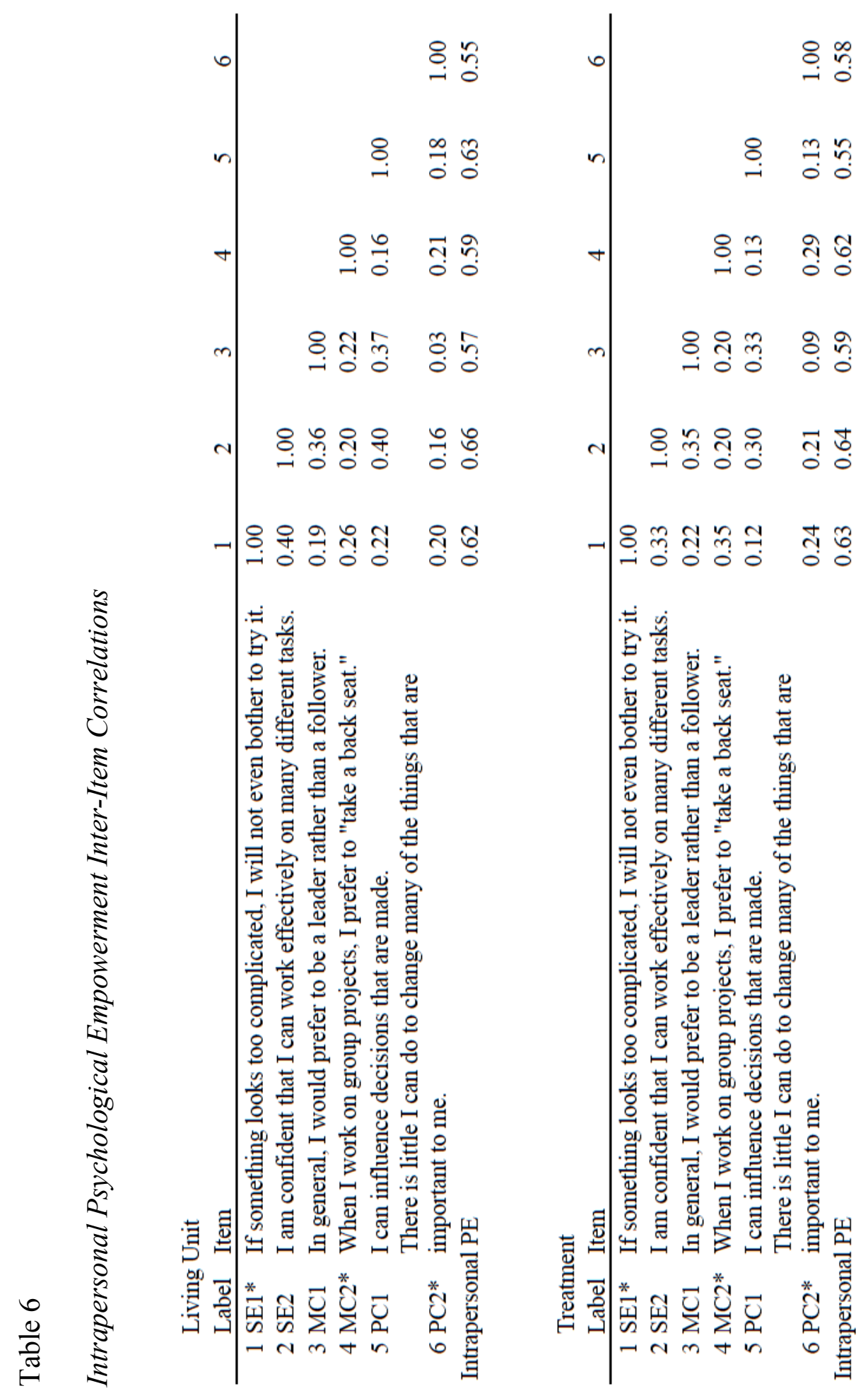




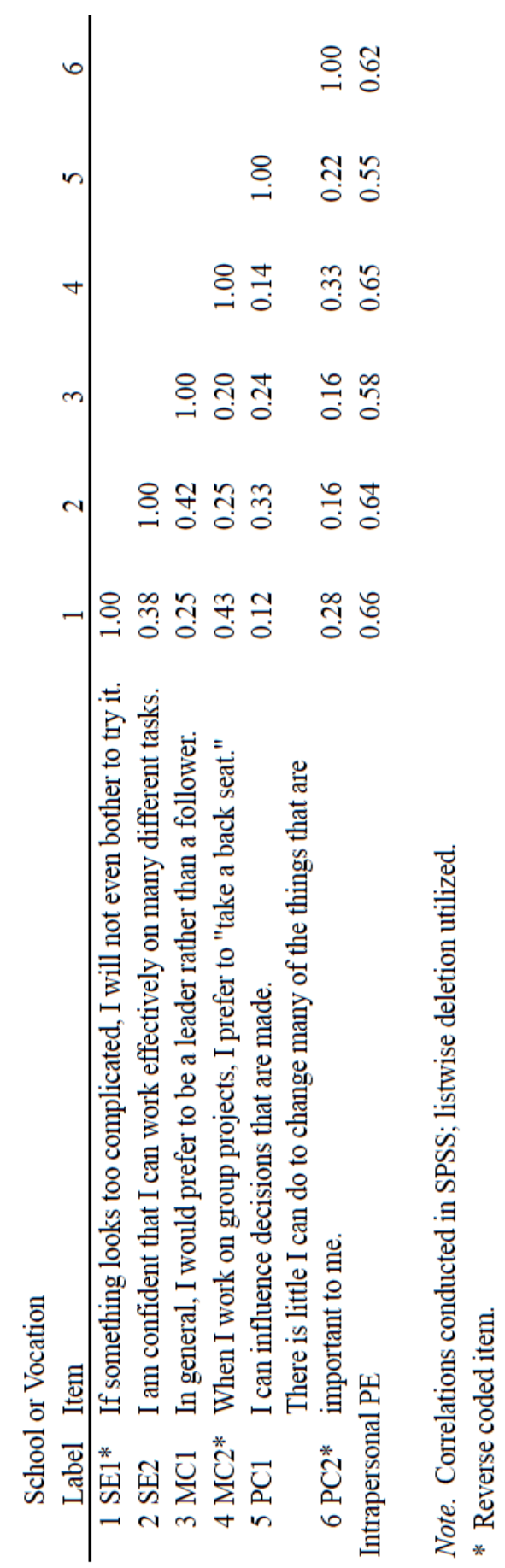




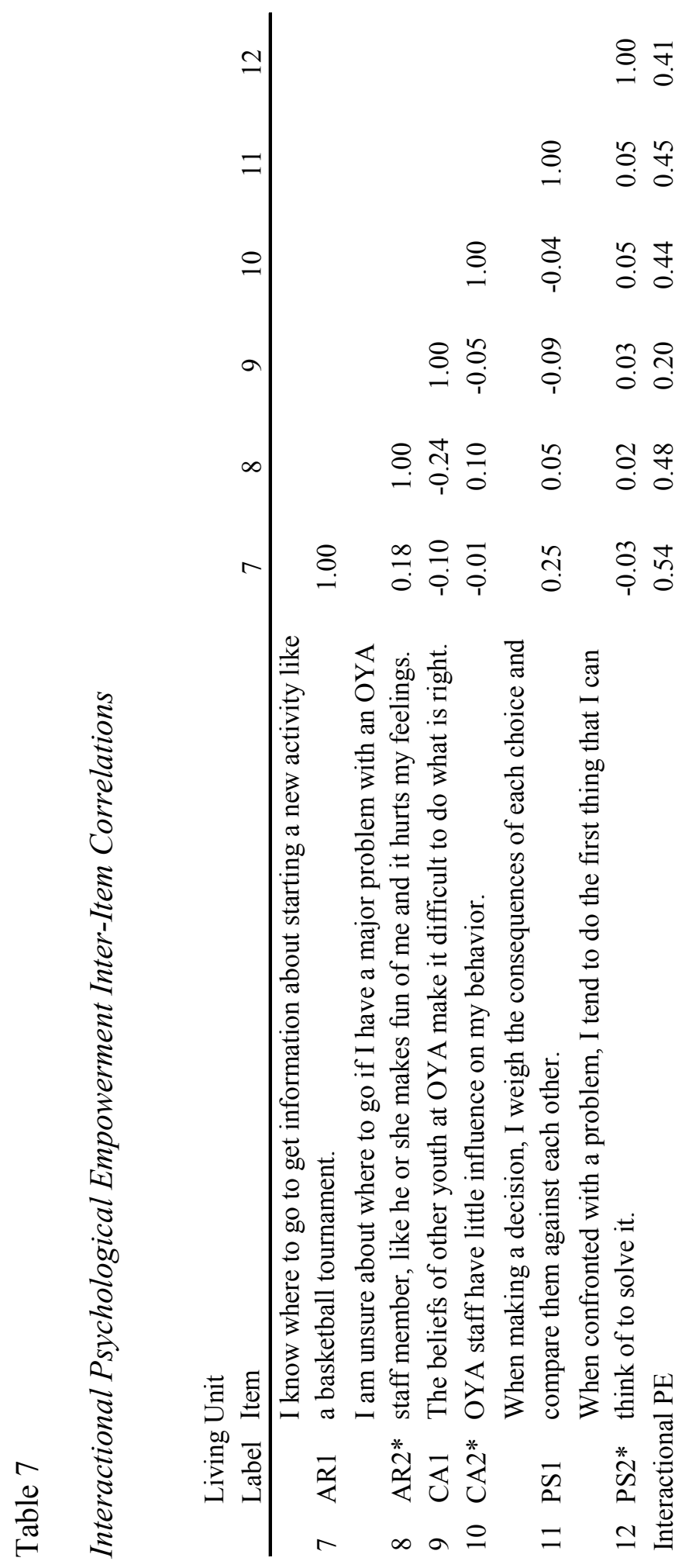




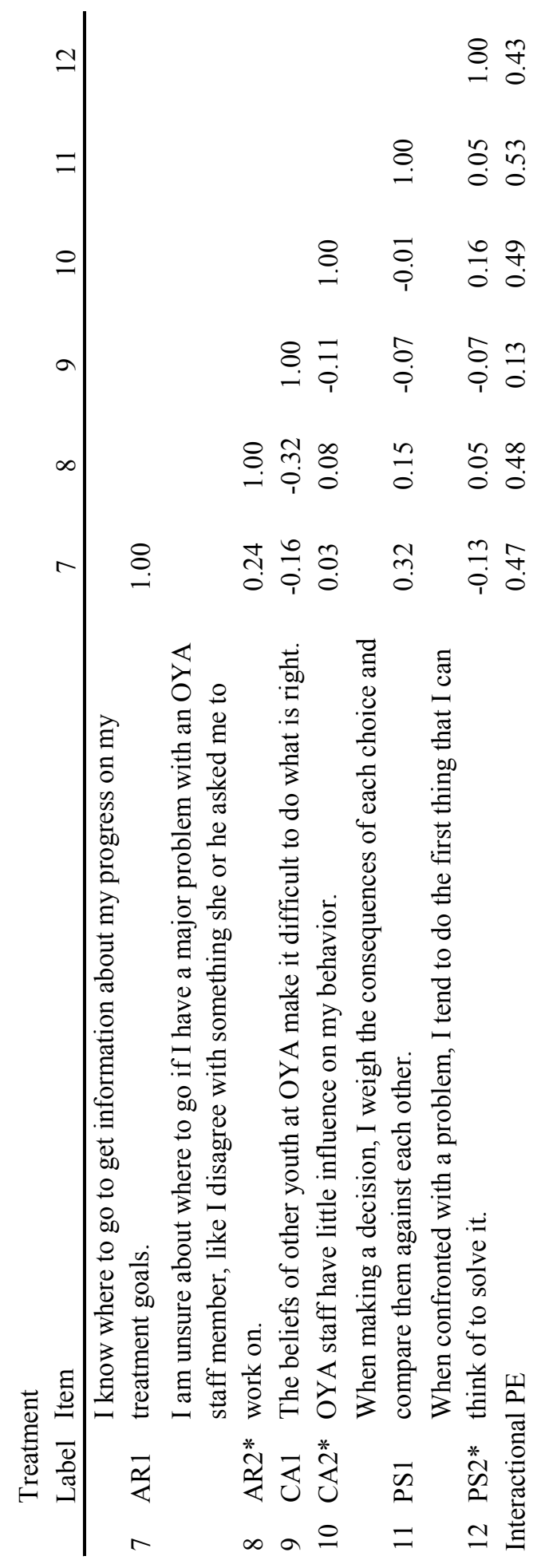




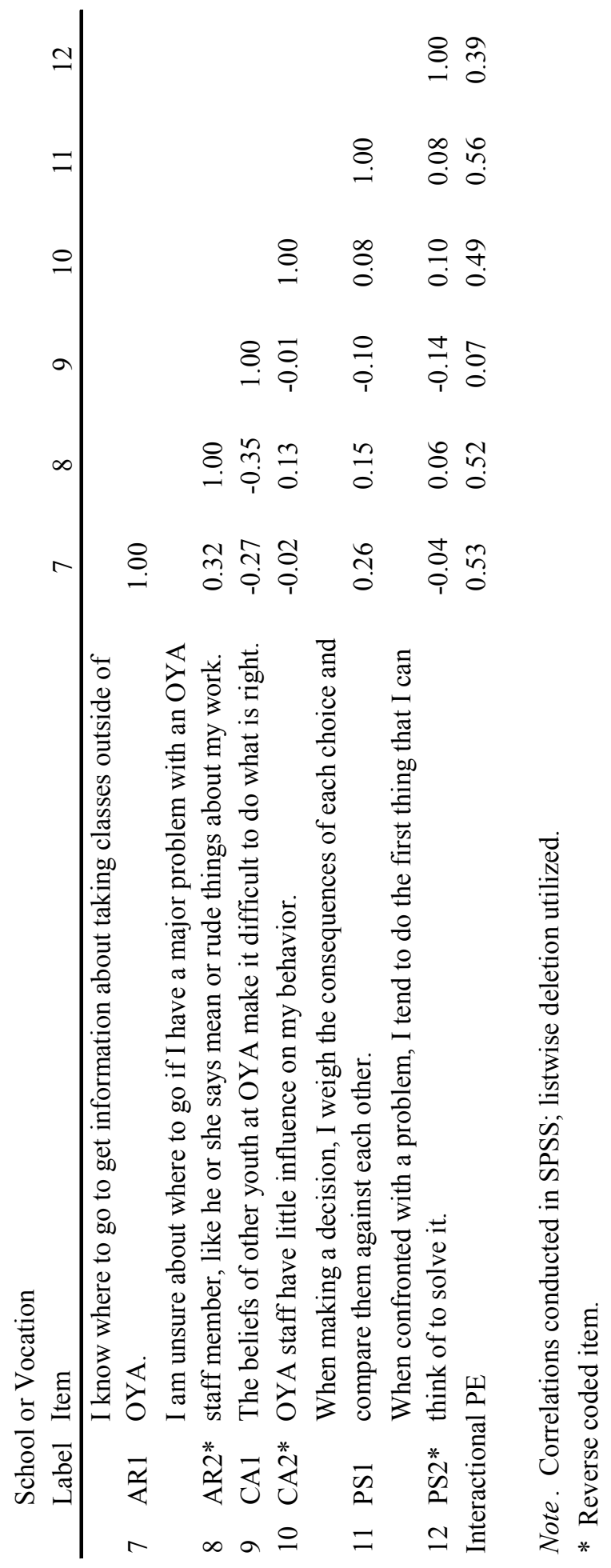




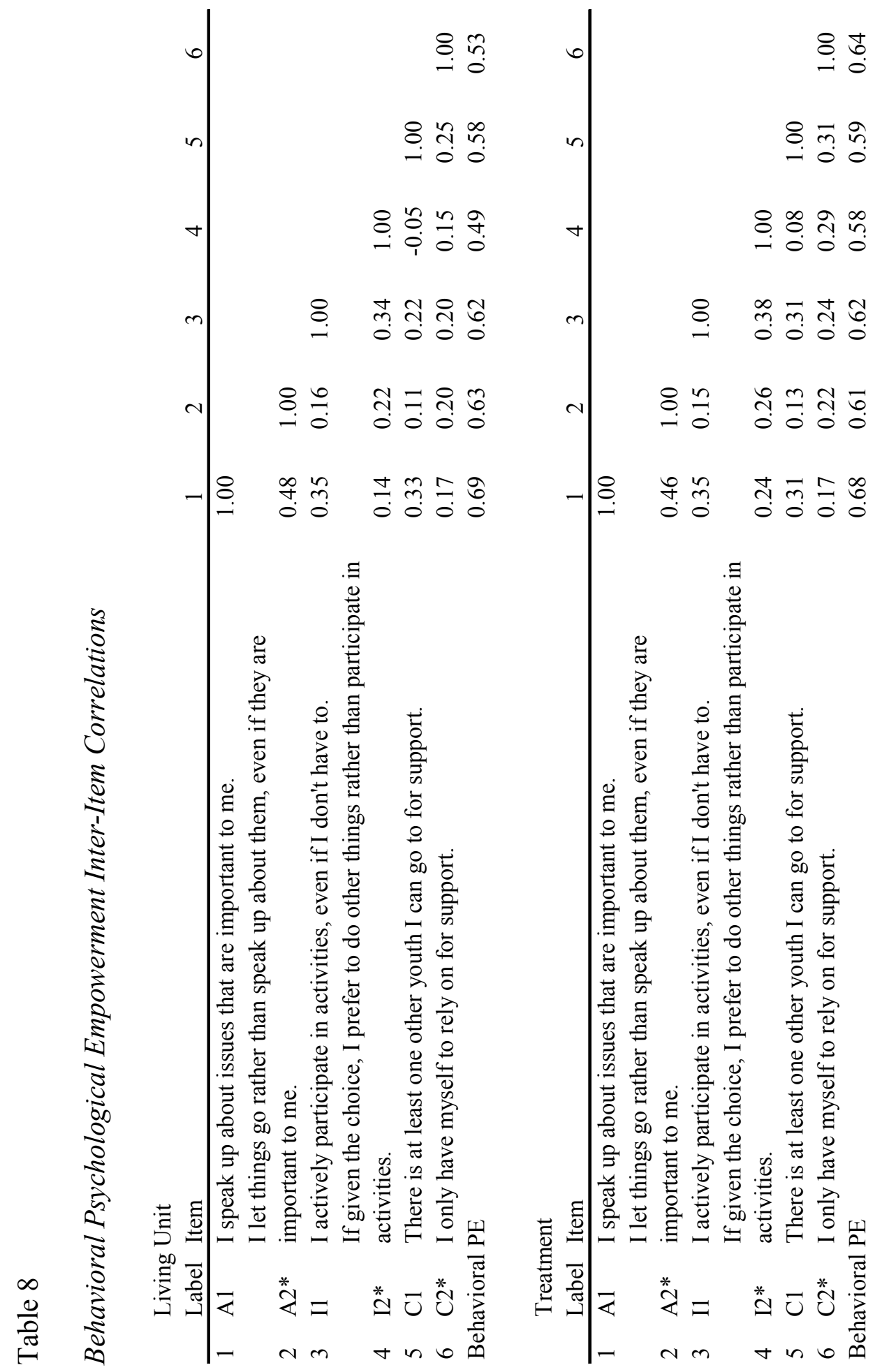




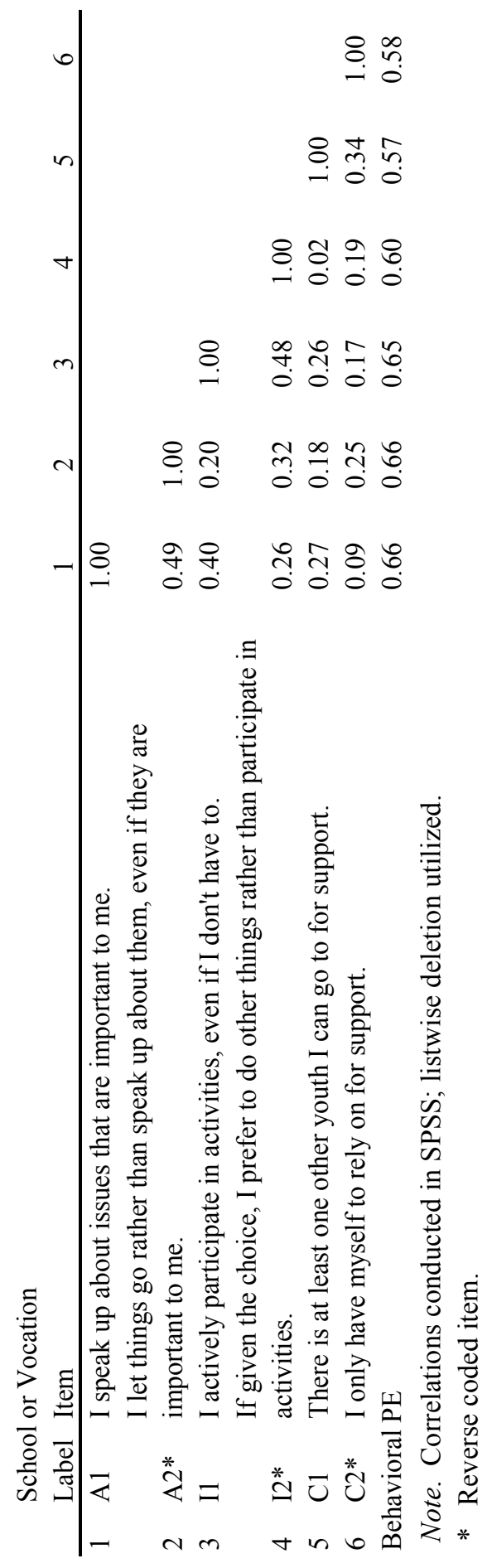




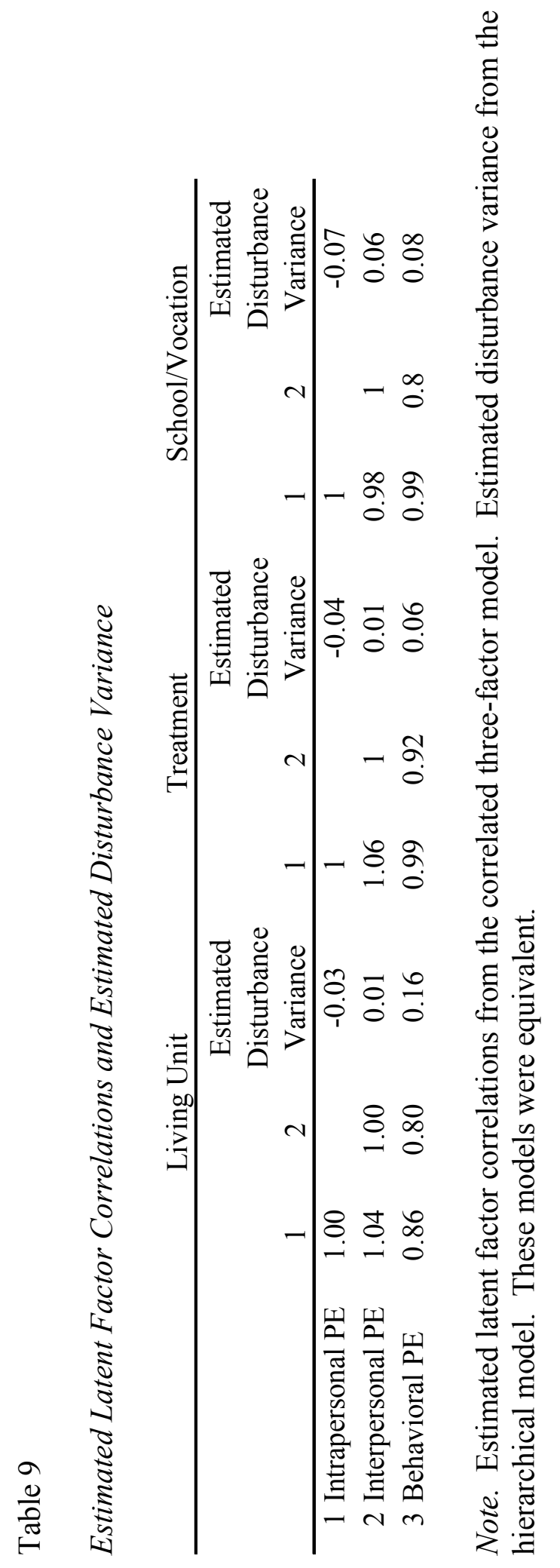




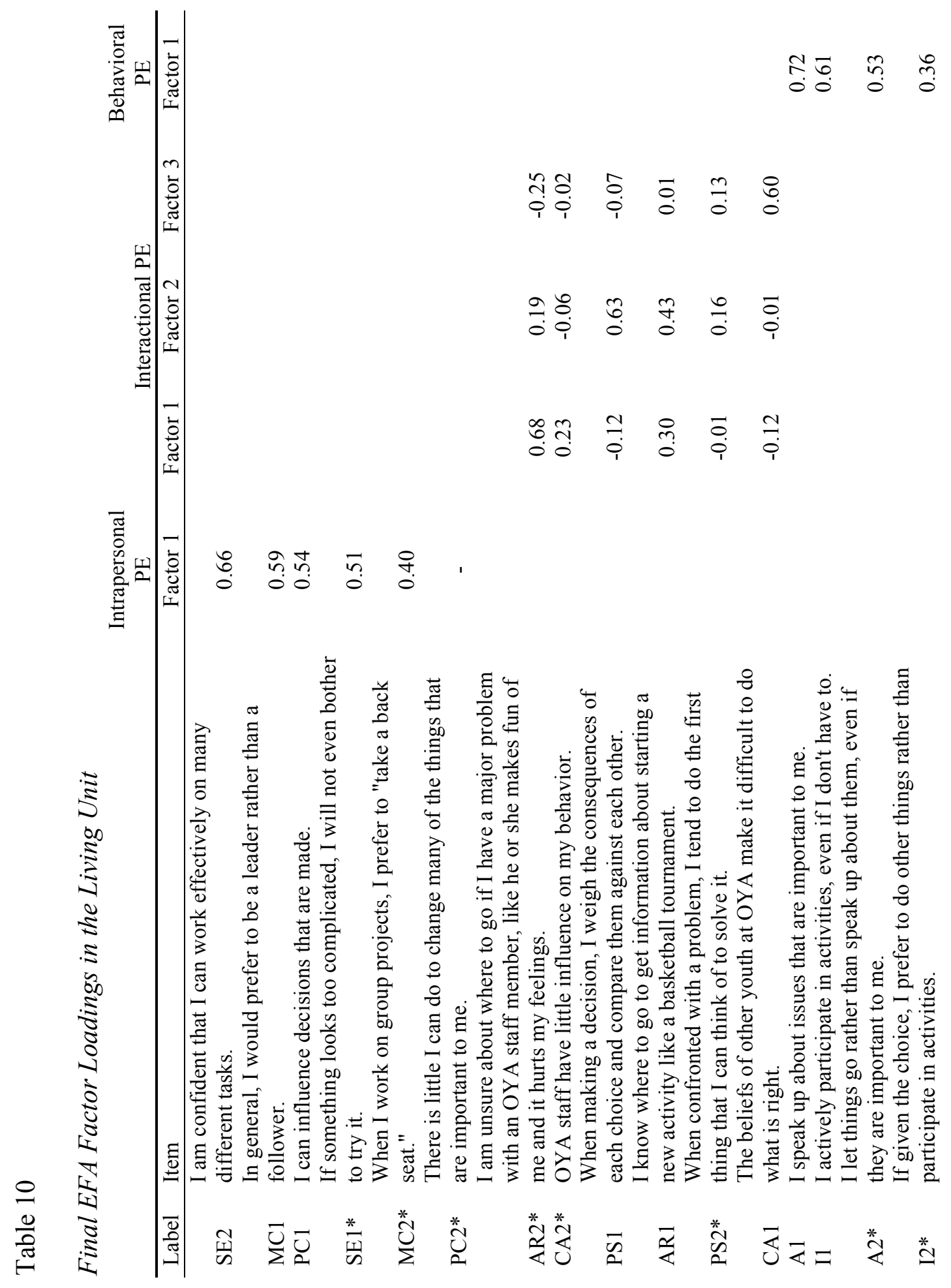


กำ

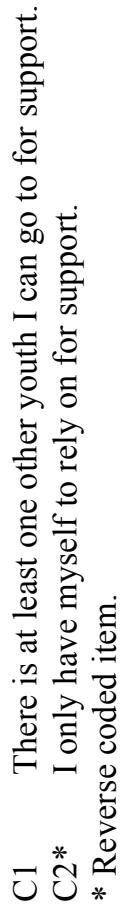




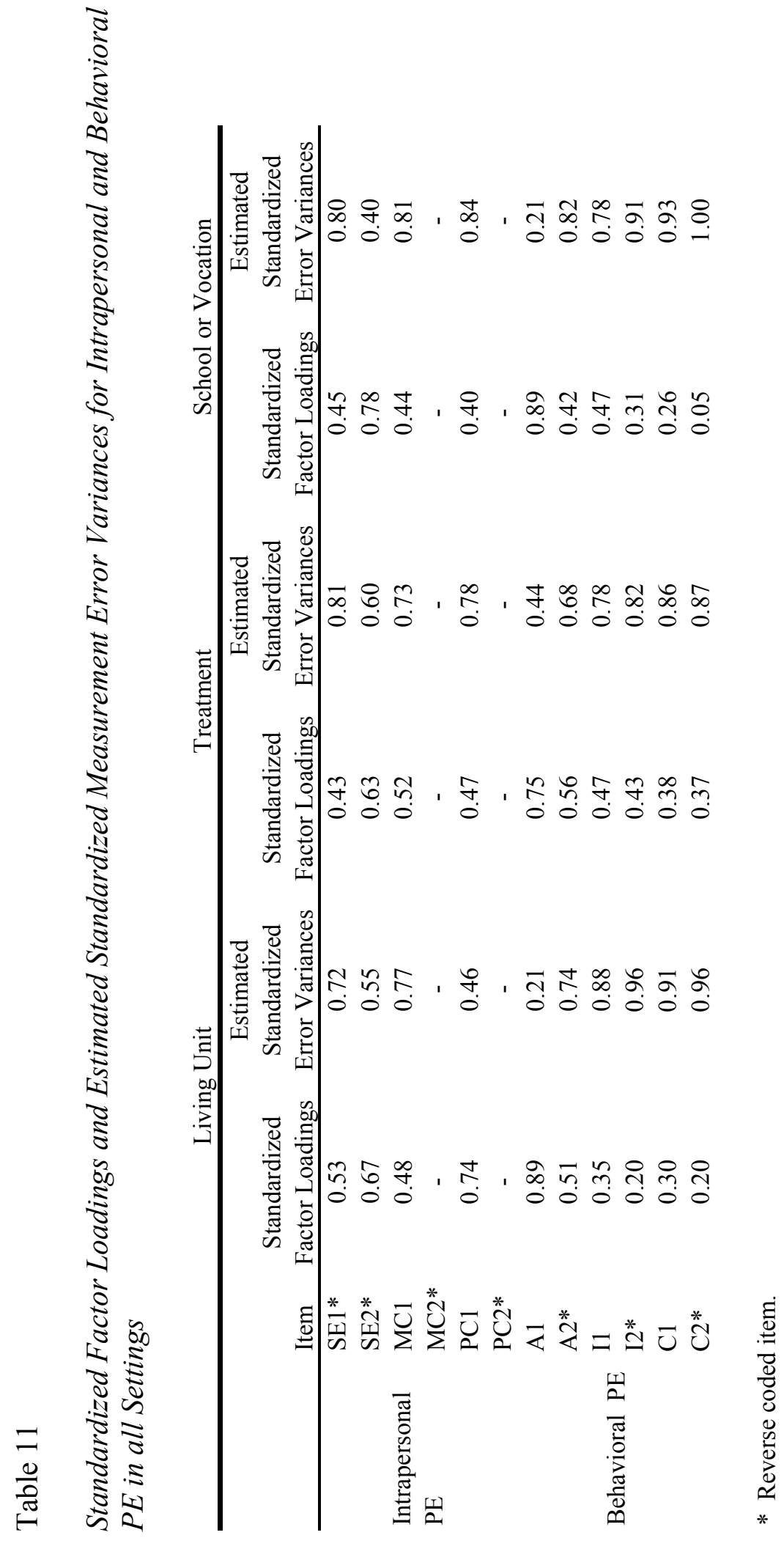




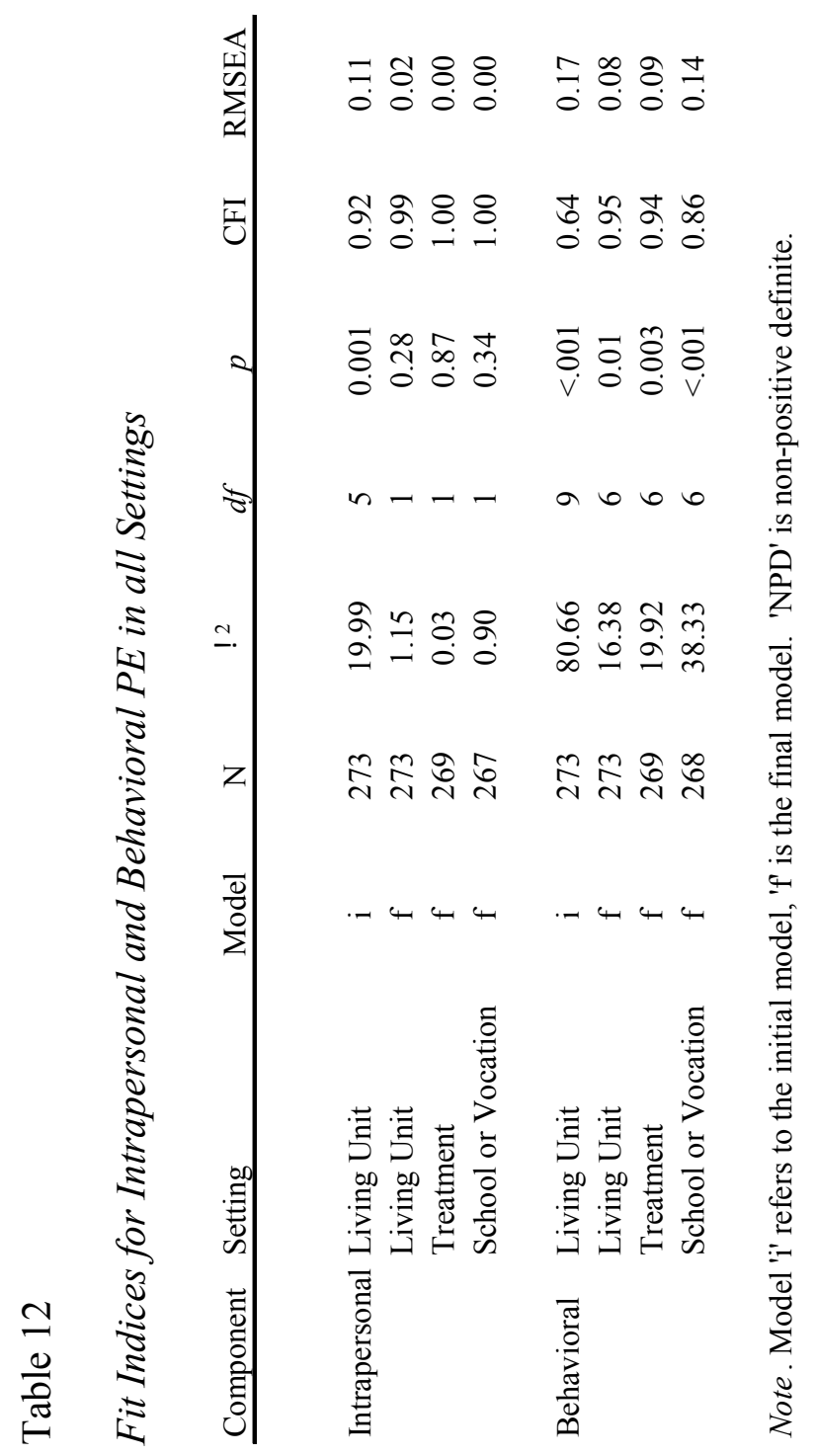




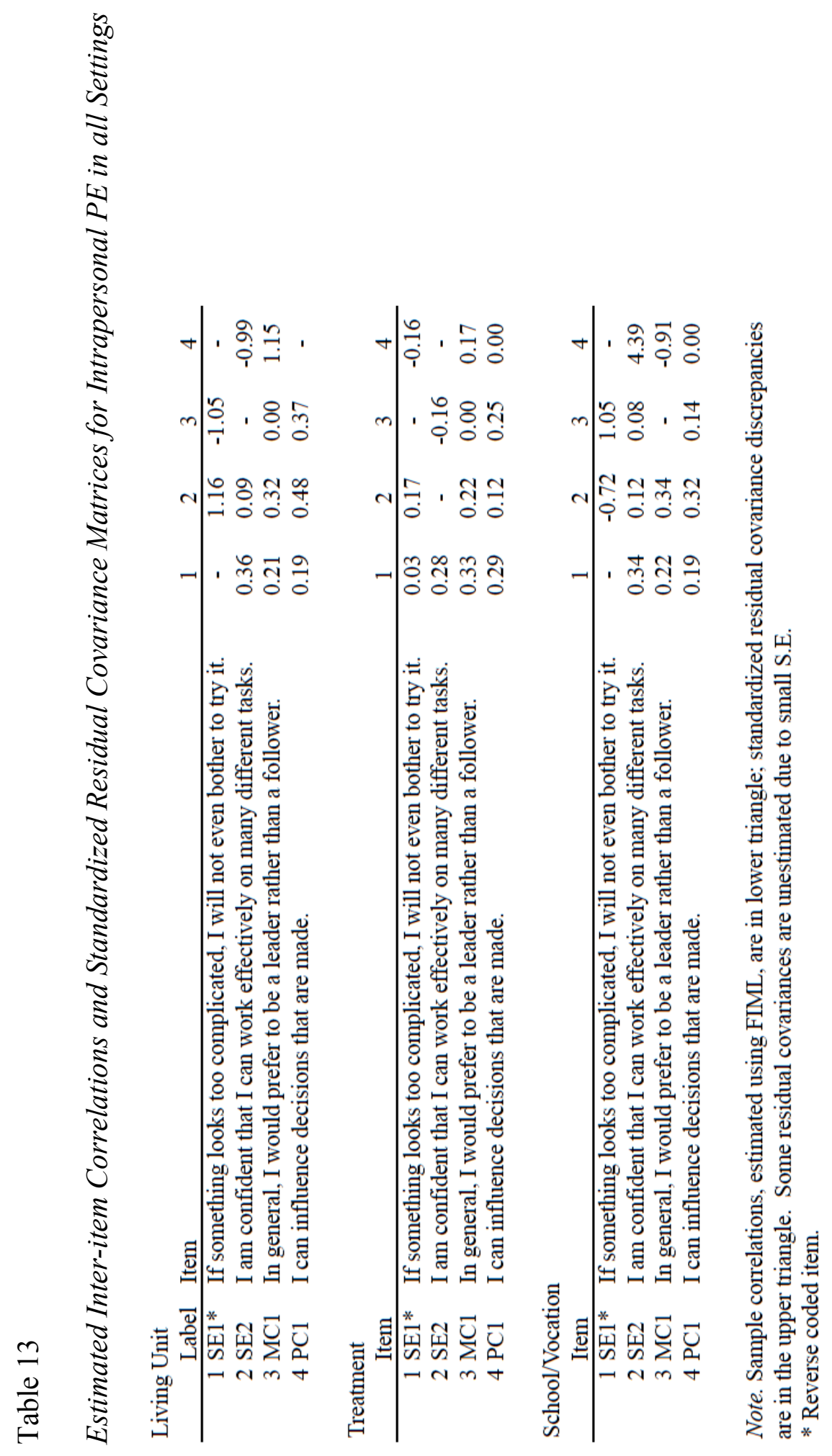


Table 14

Correlations between Intrapersonal PE in Three Settings

\begin{tabular}{llccc} 
& Setting & 1 & 2 & 3 \\
\hline 1 & Living Unit & 1 & & \\
2 & Treatment & 0.72 & 1 & \\
3 & School/Vocation & 0.69 & 0.74 & 1
\end{tabular}




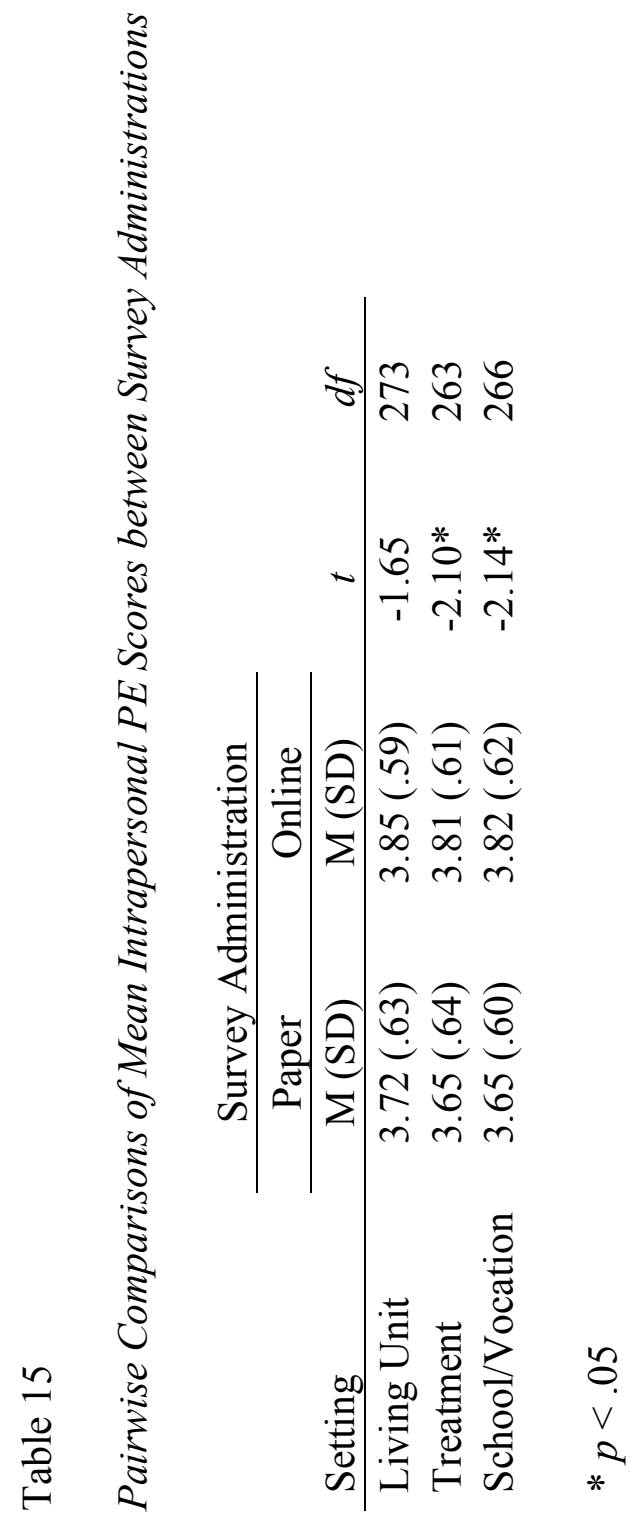




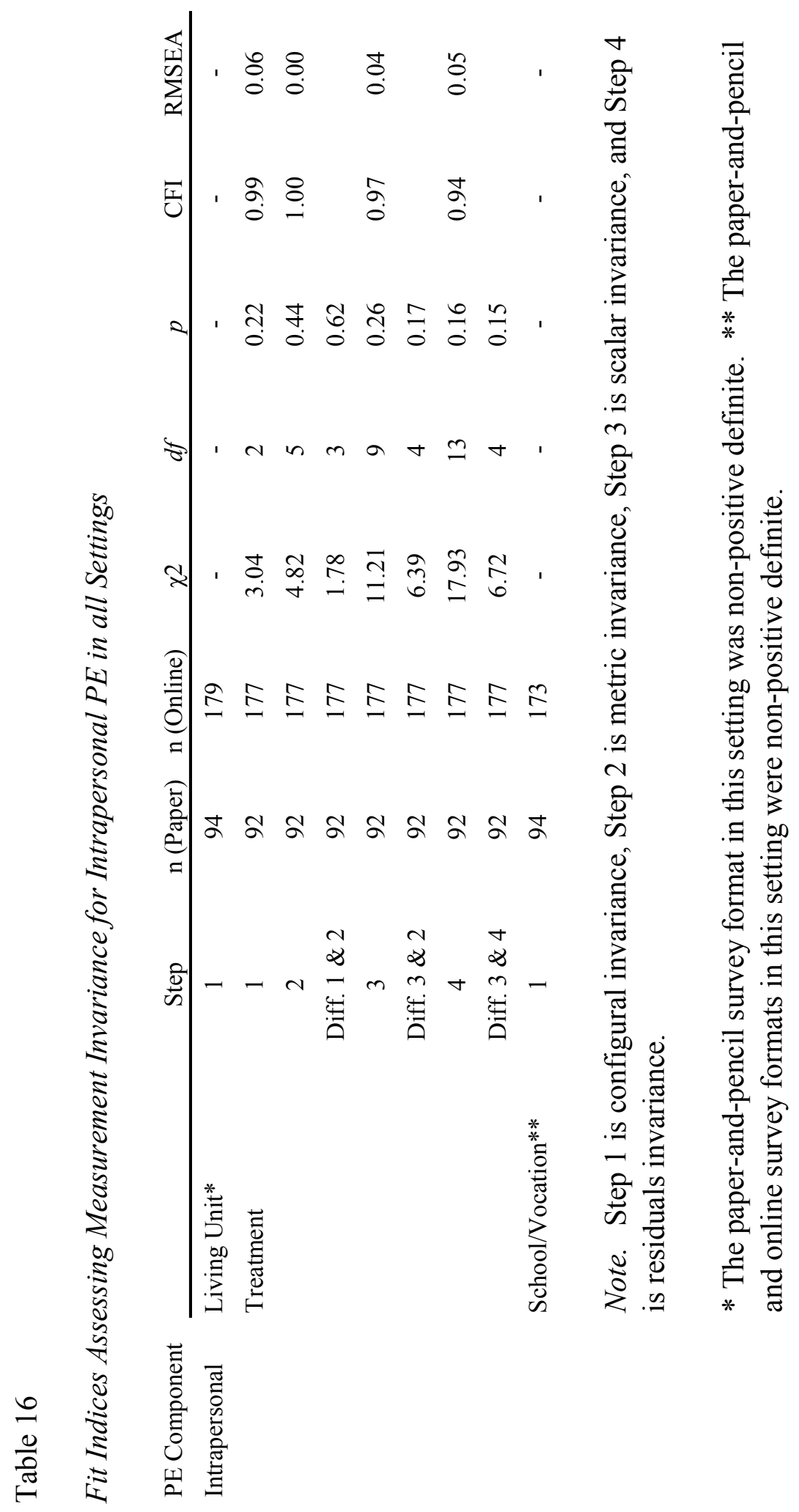




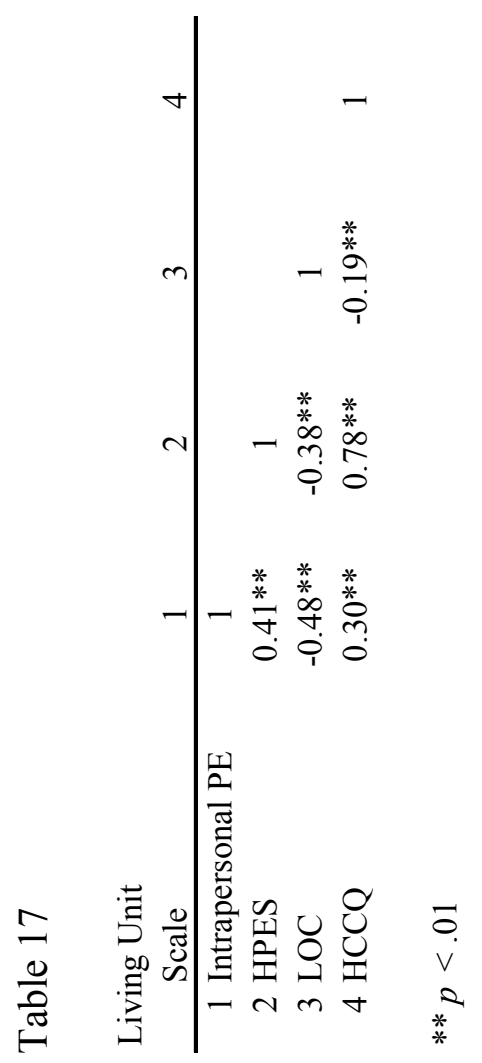




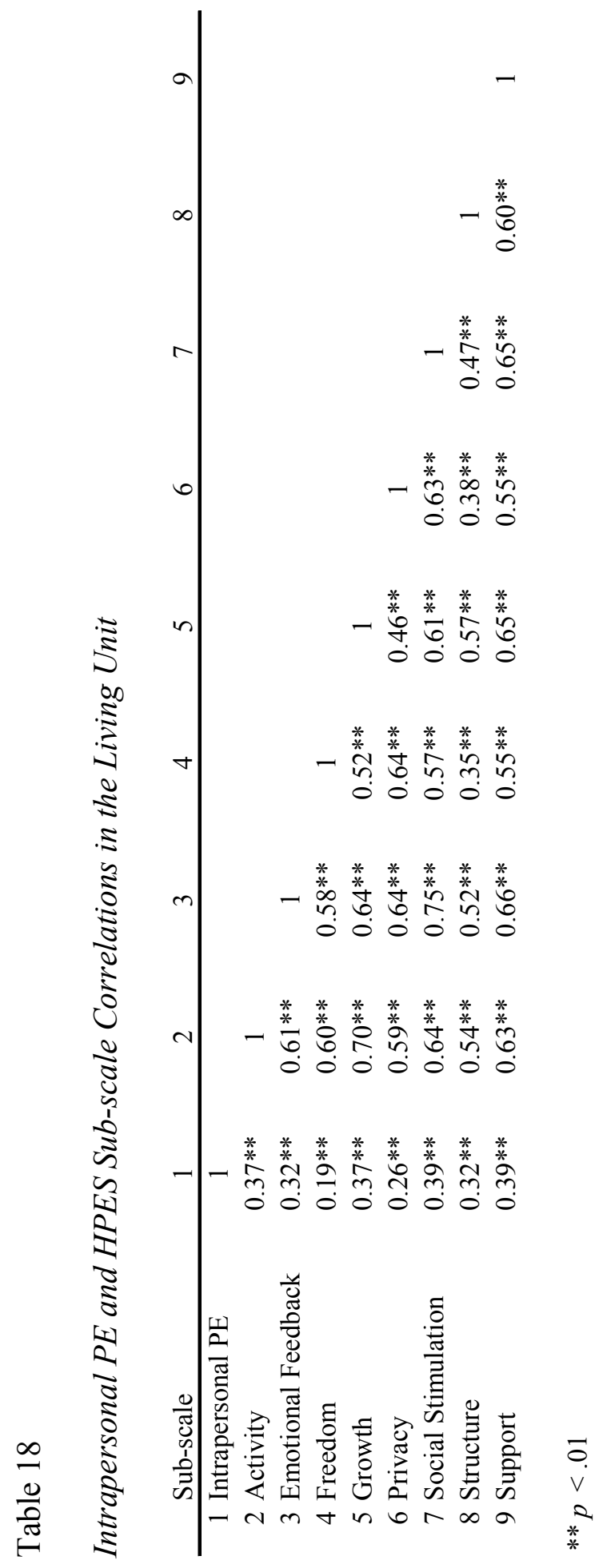




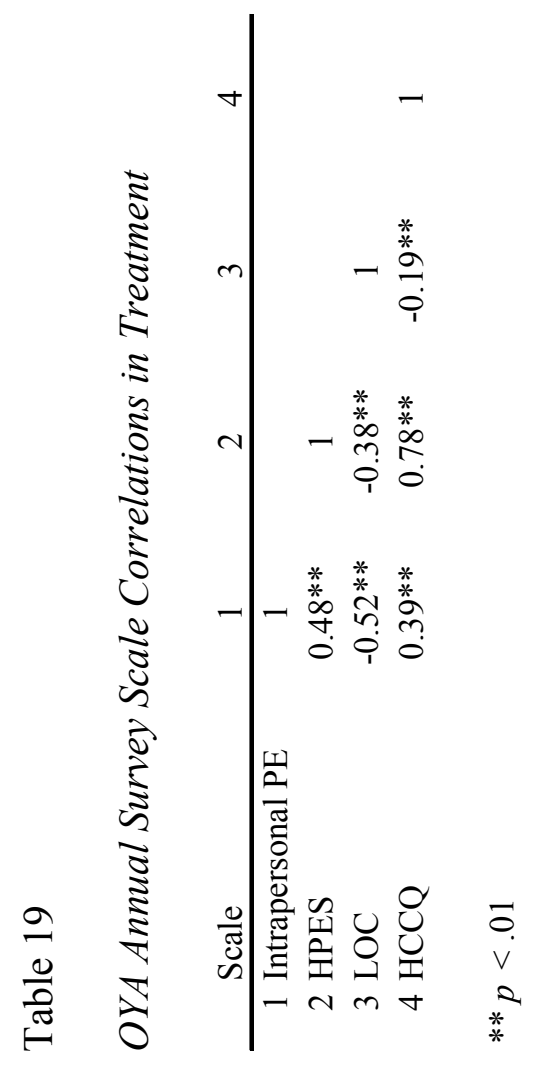




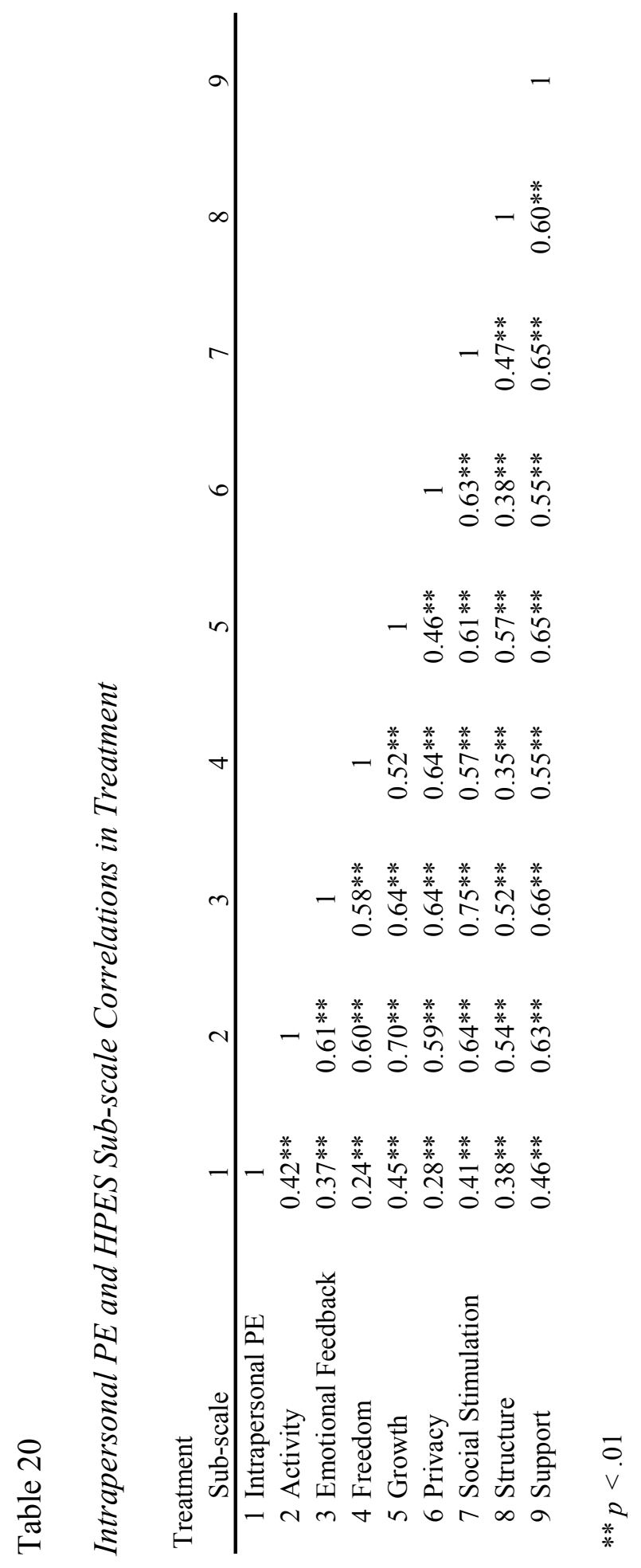




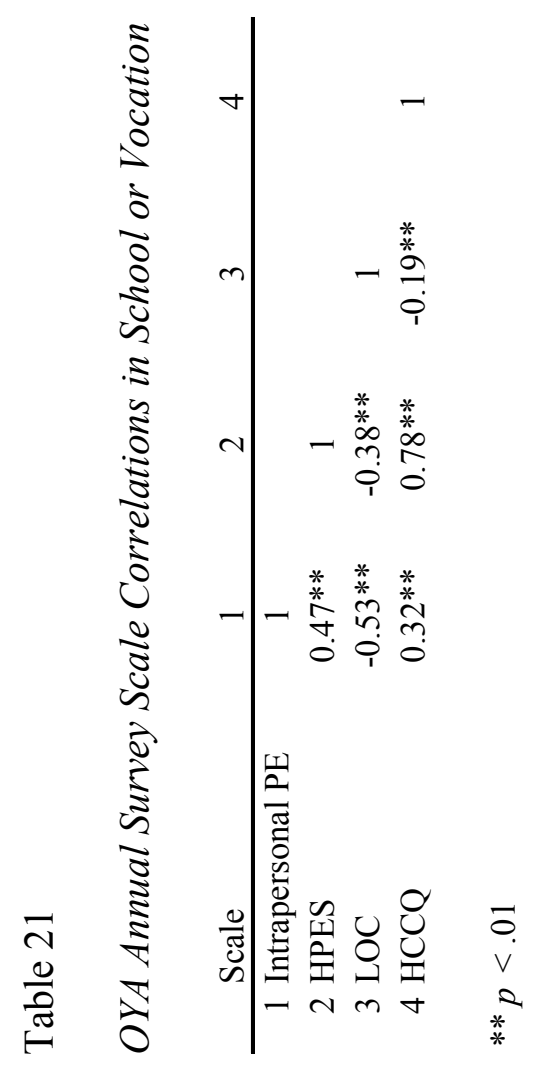




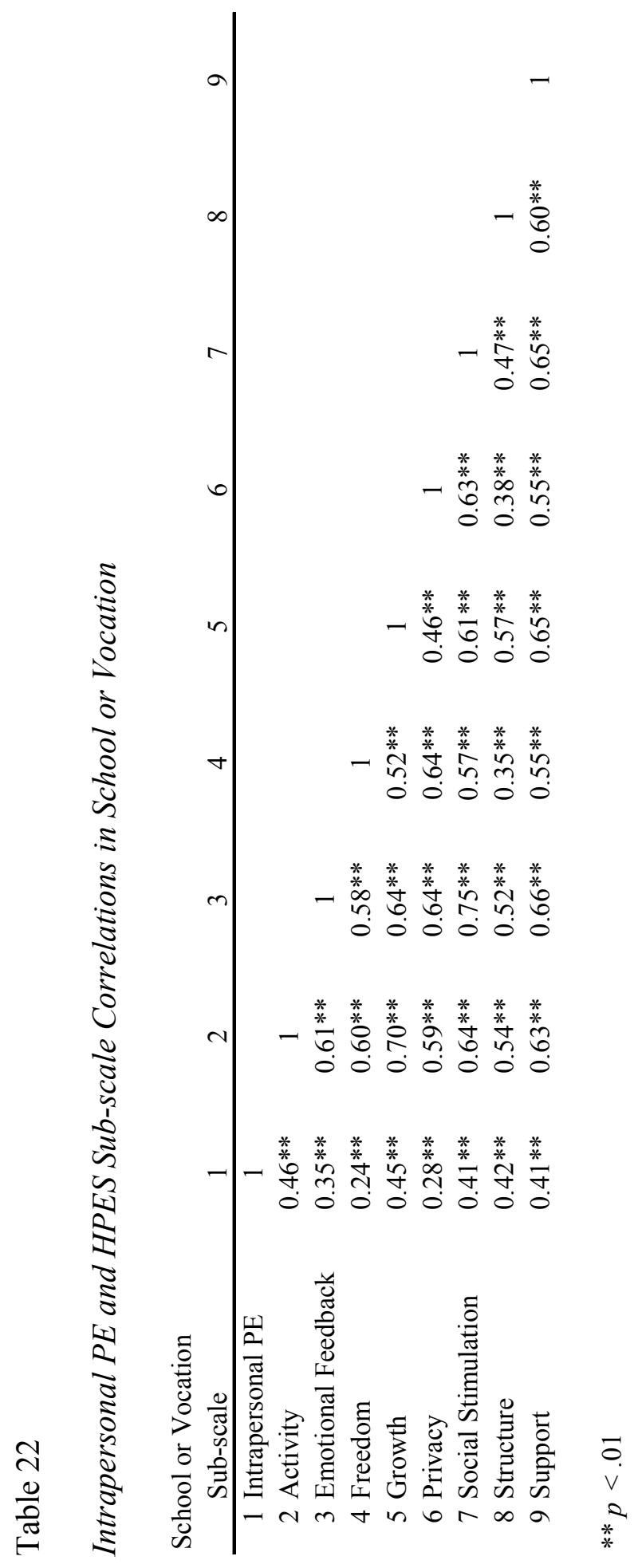




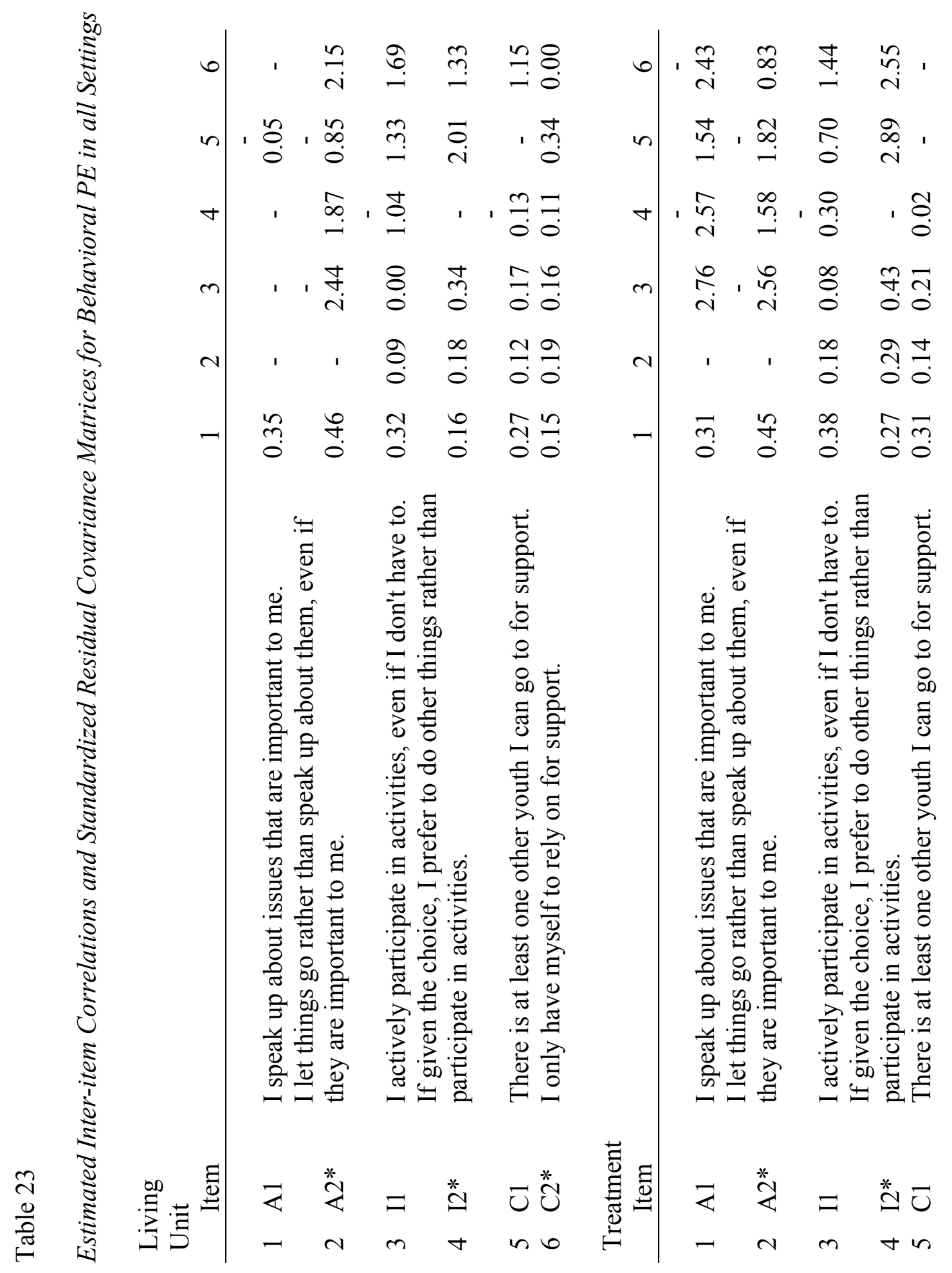




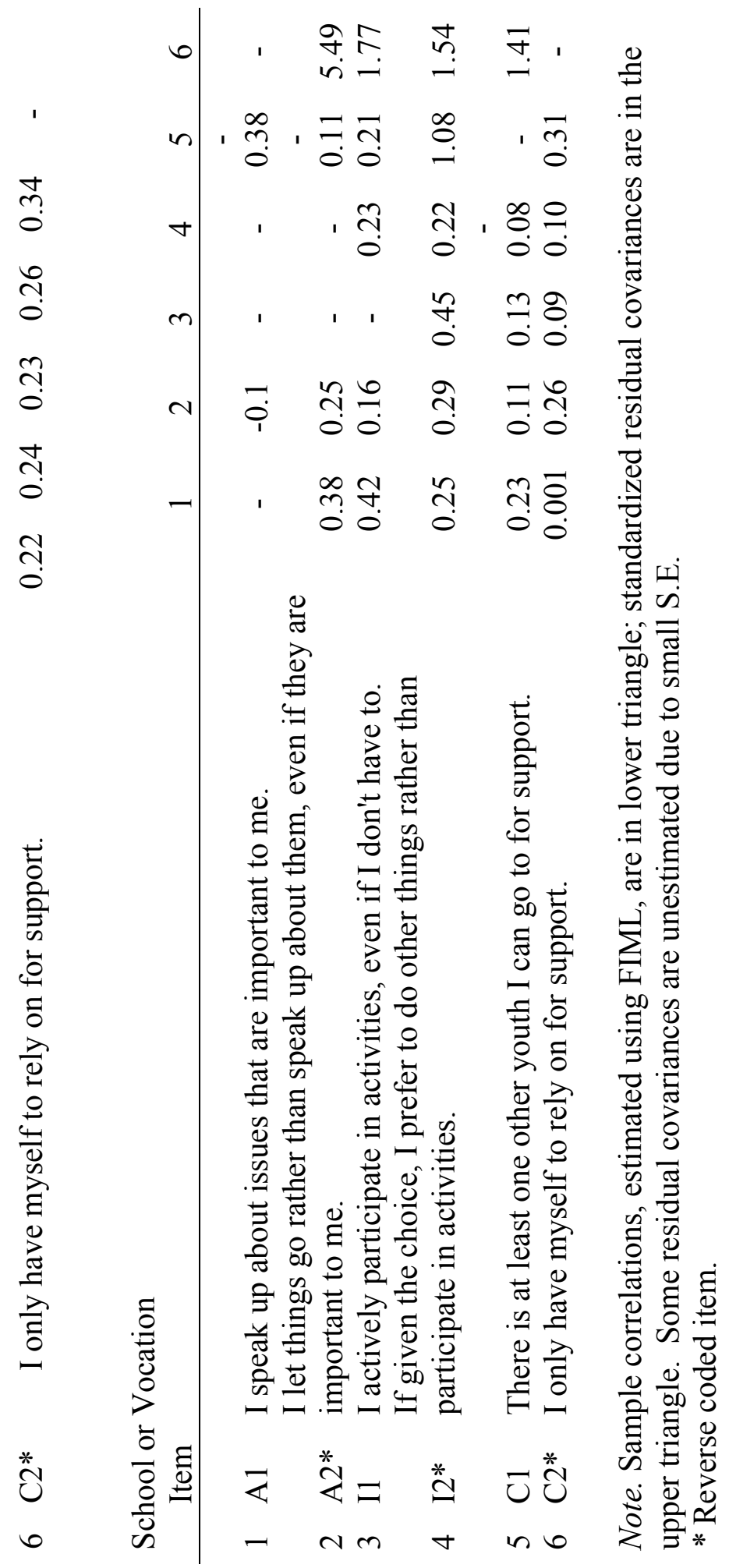


Table 24

\section{Results of HLM Predicting Behavioral Success from Intrapersonal PE}

\begin{tabular}{|c|c|c|c|c|c|c|}
\hline Domain (DV) & & $\begin{array}{l}\text { Unit } \\
\text { ICC } \\
\end{array}$ & $\begin{array}{c}\text { Facility } \\
\text { ICC }\end{array}$ & $\mathrm{B}$ & S.E. & C.I. \\
\hline \multirow[t]{8}{*}{ Education } & & 0.35 & 0.17 & & & \\
\hline & Age & & & $0.11^{* *}$ & 0.04 & $0.04,0.18$ \\
\hline & Total Time Incarcerated & & & 0.02 & 0.04 & $-0.05,0.90$ \\
\hline & Commitment Disposition & & & $0.27 * *$ & 0.10 & $0.08,0.46$ \\
\hline & Intrapersonal PE in School/Vocation & & & 0.07 & 0.07 & $-0.07,0.20$ \\
\hline & Variance Components & & & Estimate & S.E. & C.I. \\
\hline & Intercept & & & $0.27^{* *}$ & 0.09 & $0.14,0.53$ \\
\hline & & $\begin{array}{l}\text { Unit } \\
\text { ICC }\end{array}$ & $\begin{array}{c}\text { Facility } \\
\text { ICC }\end{array}$ & $\mathrm{B}$ & S.E. & C.I. \\
\hline \multirow[t]{8}{*}{ Life/Social Skills } & & 0.35 & 0.11 & & & \\
\hline & Age & & & $0.07 * *$ & 0.03 & $0.02,0.13$ \\
\hline & Total Time Incarcerated & & & 0.02 & 0.03 & $-0.03,0.08$ \\
\hline & Commitment Disposition & & & $0.45 * * *$ & 0.08 & $0.30,0.61$ \\
\hline & Intrapersonal PE in Treatment & & & 0.10 & 0.07 & $-0.03,0.24$ \\
\hline & Intrapersonal PE in Living Unit & & & 0.01 & 0.07 & $-0.13,0.14$ \\
\hline & Variance Components & & & Estimate & S.E. & C.I. \\
\hline & Intercept & & & $0.19 * *$ & 0.07 & $0.10,0.37$ \\
\hline
\end{tabular}

\begin{tabular}{|c|c|c|c|c|c|c|}
\hline & & $\begin{array}{l}\text { Unit } \\
\text { ICC }\end{array}$ & $\begin{array}{c}\text { Facility } \\
\text { ICC } \\
\end{array}$ & $\mathrm{B}$ & S.E. & C.I. \\
\hline \multirow[t]{7}{*}{ Offense-Specific } & & 0.44 & 0.22 & & & \\
\hline & Age & & & $0.06^{*}$ & 0.03 & $0.01,0.12$ \\
\hline & Total Time Incarcerated & & & 0.03 & 0.03 & $-0.03,0.08$ \\
\hline & Commitment Disposition & & & $0.42 * * *$ & 0.08 & $0.26,0.58$ \\
\hline & Intrapersonal PE in Treatment & & & $0.13 *$ & 0.05 & $0.03,0.23$ \\
\hline & Variance Components & & & Estimate & S.E. & C.I. \\
\hline & Intercept & & & $0.26 * *$ & 0.09 & $0.13,0.51$ \\
\hline
\end{tabular}

\begin{tabular}{|c|c|c|c|c|c|c|}
\hline & & $\begin{array}{l}\text { Unit } \\
\text { ICC }\end{array}$ & $\begin{array}{c}\text { Facility } \\
\text { ICC }\end{array}$ & B & S.E. & C.I. \\
\hline \multirow[t]{7}{*}{ Mental Health } & & 0.37 & 0.18 & & & \\
\hline & Age & & & $0.14 * *$ & 0.04 & $0.06,0.22$ \\
\hline & Total Time Incarcerated & & & -0.04 & 0.04 & $-0.12,0.04$ \\
\hline & Commitment Disposition & & & $0.40 * *$ & 0.11 & $0.17,0.62$ \\
\hline & Intrapersonal PE in Treatment & & & 0.07 & 0.07 & $-0.08,0.21$ \\
\hline & Variance Components & & & Estimate & S.E. & C.I. \\
\hline & Intercept & & & $0.23 *$ & 0.10 & $0.10,0.52$ \\
\hline
\end{tabular}




\begin{tabular}{|c|c|c|c|c|c|c|}
\hline & & $\begin{array}{l}\text { Unit } \\
\text { ICC } \\
\end{array}$ & $\begin{array}{c}\text { Facility } \\
\text { ICC } \\
\end{array}$ & $\mathrm{B}$ & S.E. & C.I. \\
\hline \multirow[t]{7}{*}{ Vocational } & & 0.45 & 0.22 & & & \\
\hline & Age & & & $0.12 * *$ & 0.04 & $0.05,0.19$ \\
\hline & Total Time Incarcerated & & & -0.01 & 0.03 & $-0.08,0.05$ \\
\hline & Commitment Disposition & & & $0.26^{* *}$ & 0.09 & $0.08,0.43$ \\
\hline & Intrapersonal PE in School/Vocation & & & $0.13 *$ & 0.06 & $0.01,0.25$ \\
\hline & Variance Components & & & Estimate & S.E. & C.I. \\
\hline & Intercept & & & $0.26^{* *}$ & 0.09 & $0.13,0.52$ \\
\hline
\end{tabular}

Note. The grouping variable in analyses was unit; facility ICC is provided as a comparison.

$* \mathrm{p}<.05 . * * \mathrm{p}<.01 . * * * \mathrm{p}<.001$ 


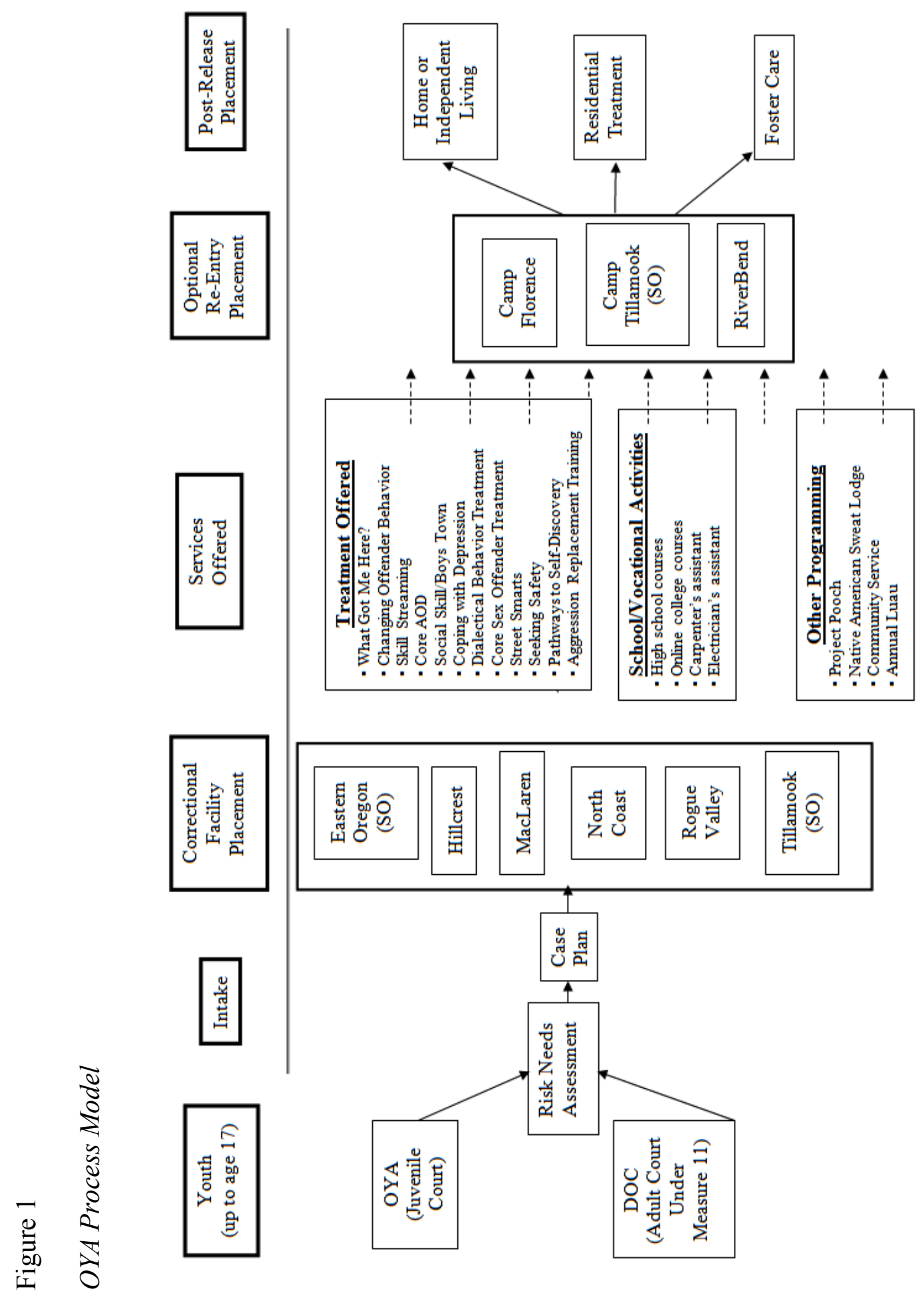




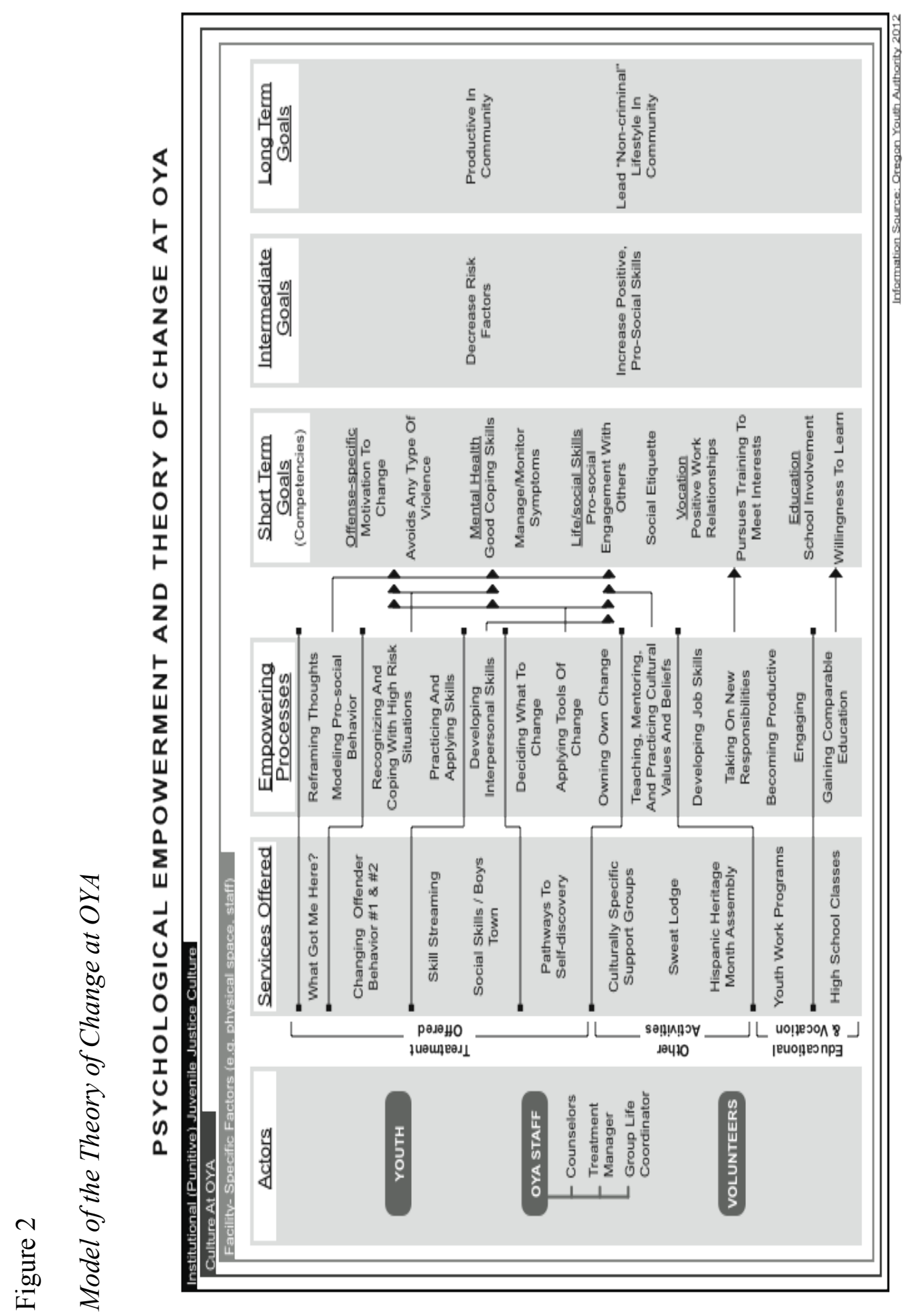




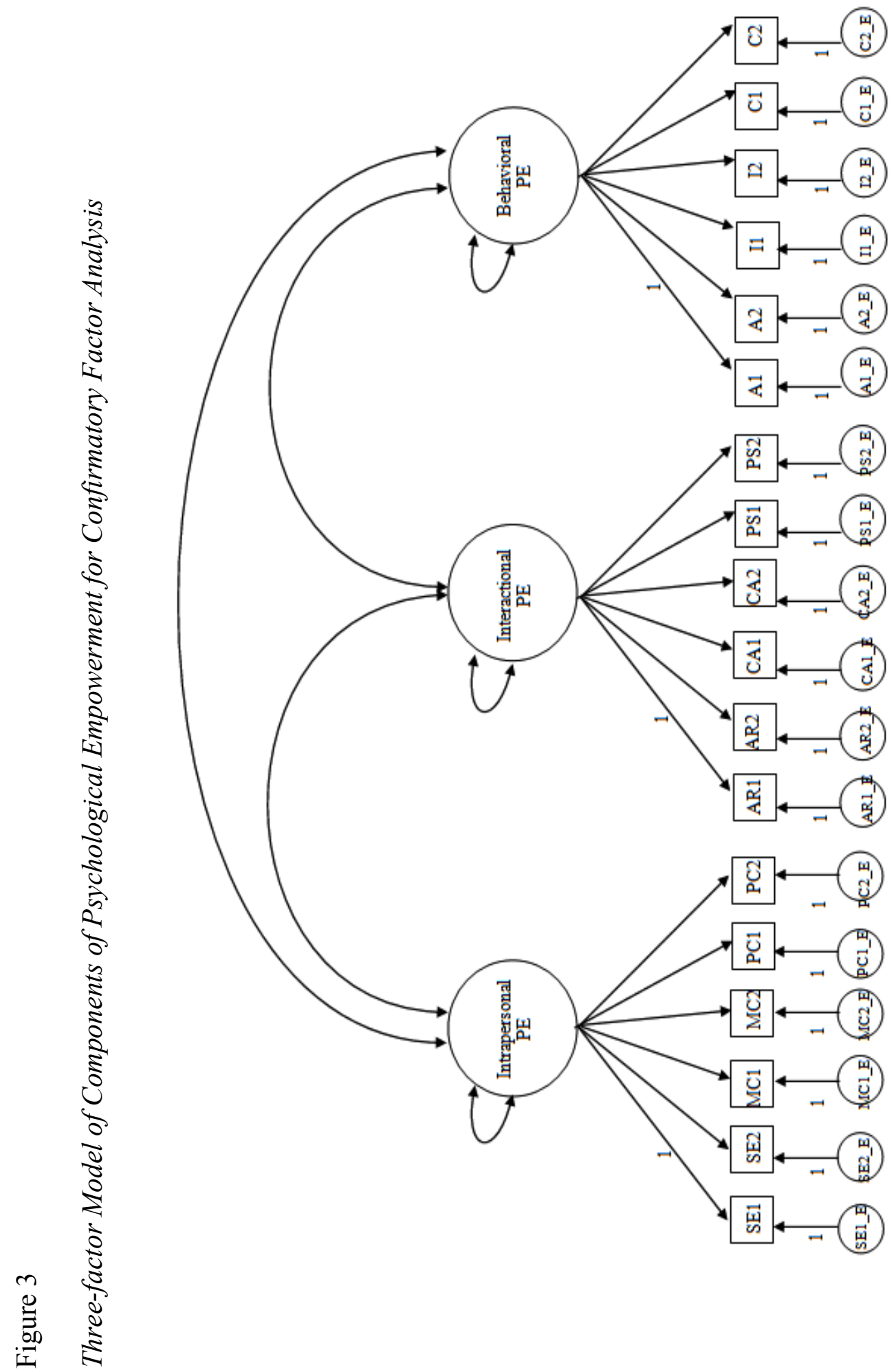


Figure 4

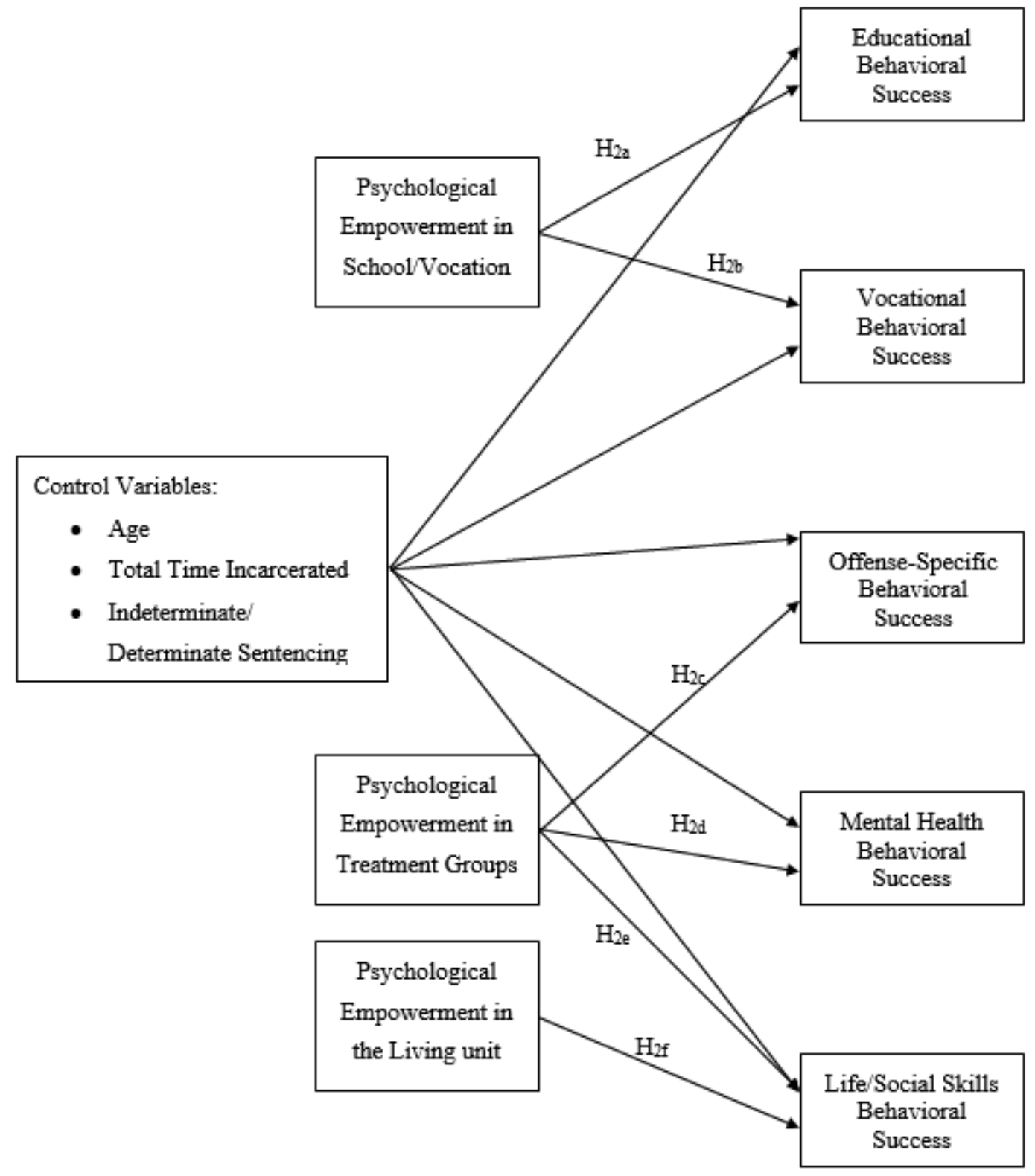




\section{Figure 5}

Single-factor Model for Intrapersonal PE for Initial Confirmatory Factor Analysis in Living Unit

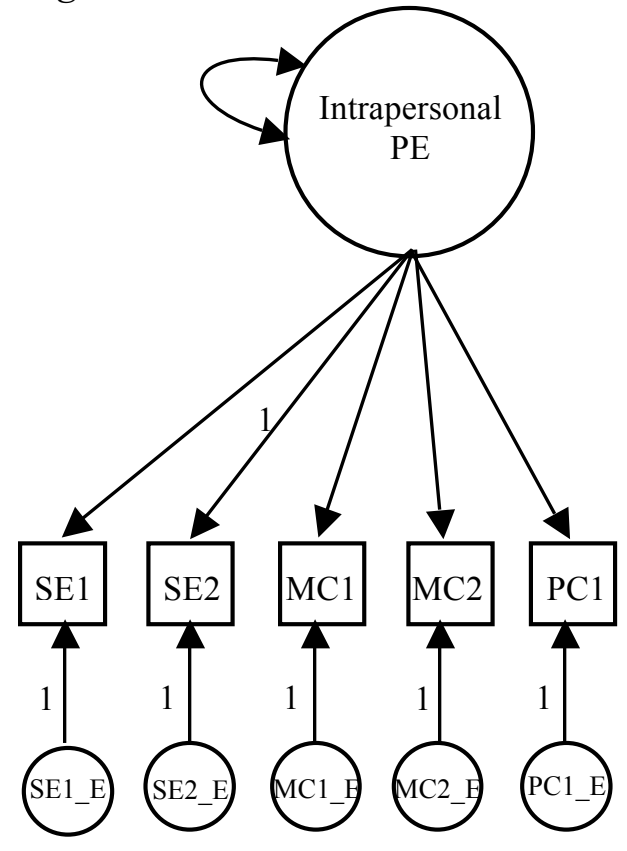


Figure 6

Final Single-factor Model for Intrapersonal PE

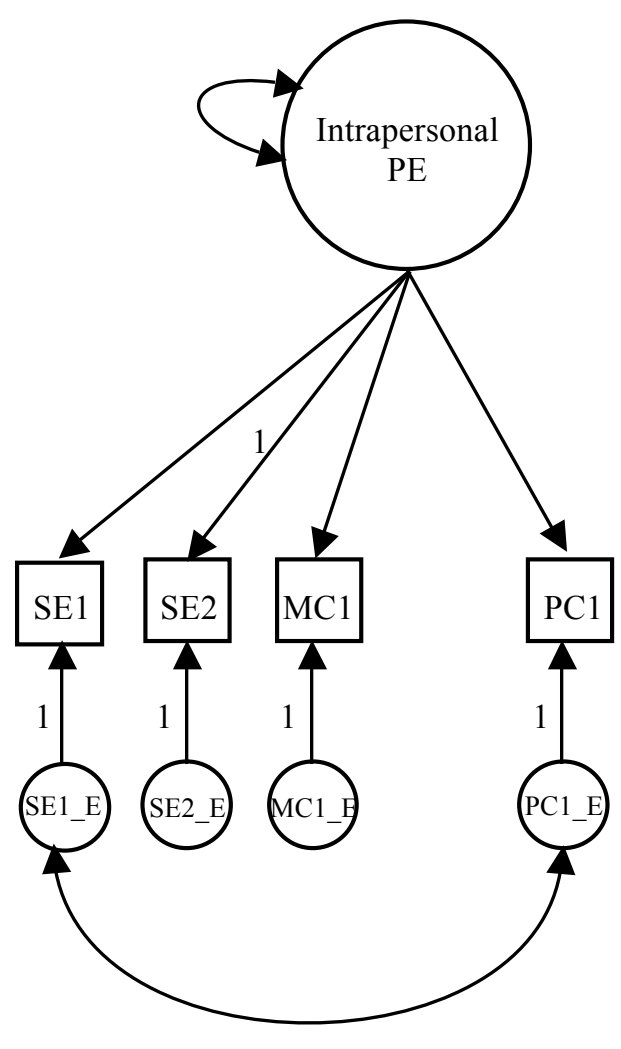


Figure 7

Single-factor Model of Behavioral PE for Initial Confirmatory Factor Analysis

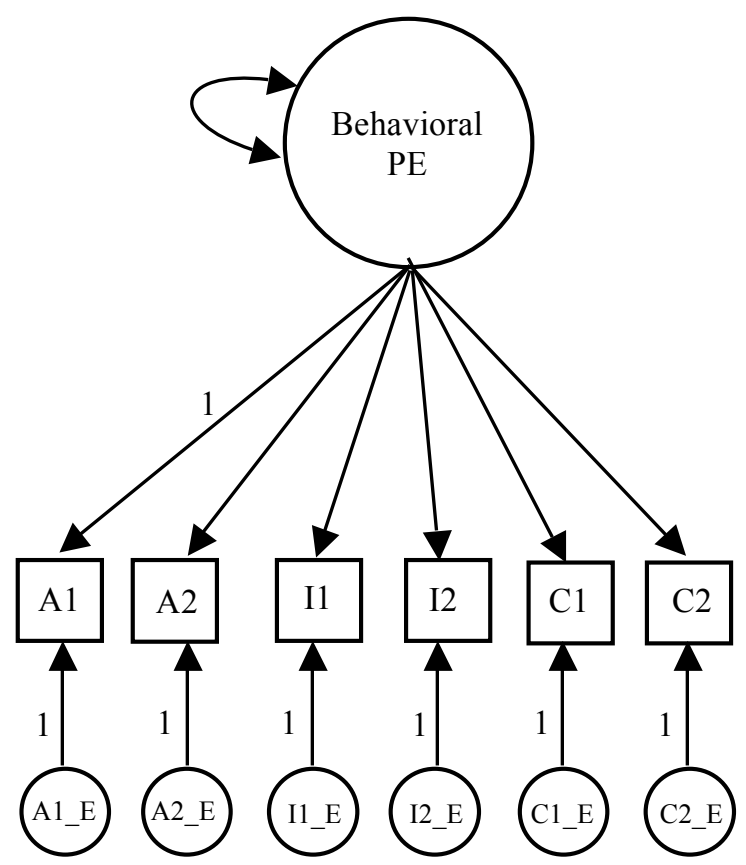


Figure 8

Final Single-factor Model of Behavioral PE

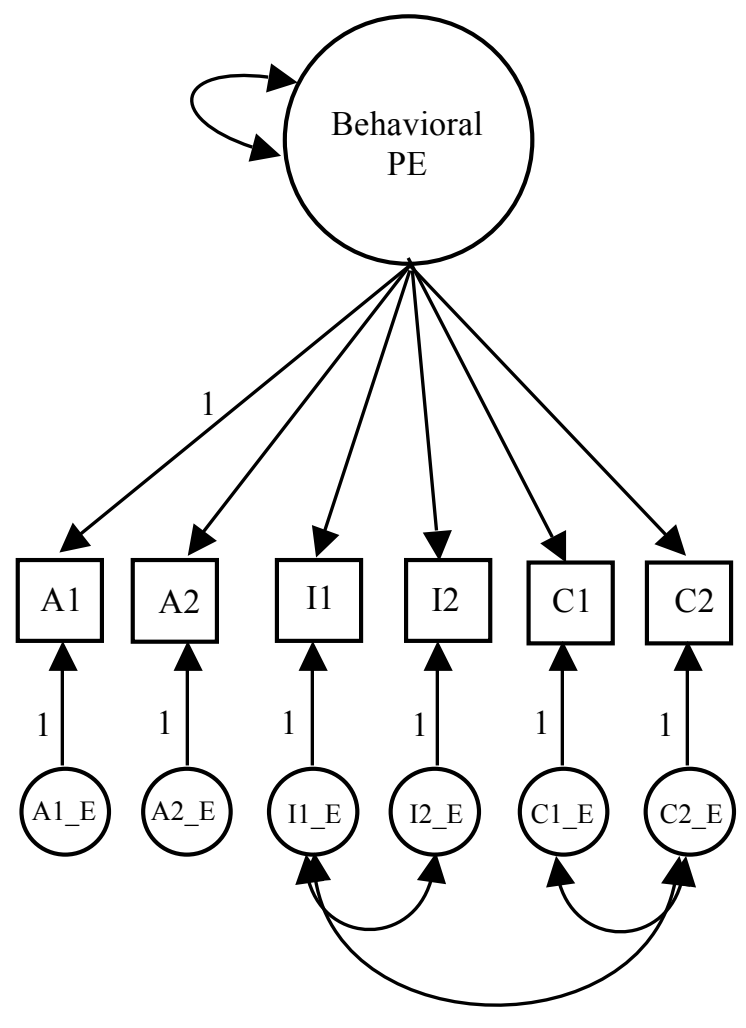




\section{References}

Abrams, L.S. (2006). From corrections to community: Youth offenders' perceptions of the challenges of transition. Journal of Offender Rehabilitation, 44(2/3), 31-53.

Akey, T.M., Marquis, J.G., \& Ross, M.E. (2000). Validation of scores on the psychological empowerment scale: A measure of empowerment for parents of children with a disability. Educational and Psychological Measurement, 60(3), 419-438.

Almost, J. \& Laschinger, H.K.S. (2002). Workplace empowerment, collaborative work relationships, and job strain in nurse practitioners. Journal of the American Academy of Nurse Practitioners, 14(9), 408-420.

Altschuler, D.M. \& Brash, R. (2004). Adolescent and teenage offenders confronting the challenges and opportunities of reentry. Youth Violence and Juvenile Justice, 2(1), 72-87.

Amirkhan, J.H. (1990). A factor analytically derived measure of coping: The coping strategy indicator. Journal of Personality and Social Psychology, 59(5), 10661074.

Amnesty International (2005). The rest of their lives: Life without parole for child offenders in the United States. Human Rights Watch. Retrieved 14 April 2013 from http:/www.amnestyusa.org/sites/default/files/pdfs/therestoftheirlives.pdf.

Andres-Hyman, R. C., Forrester, A., Achara-Abrahams, I., Lauricella, M.L., \& Rowe, M. (2007). Oppression and empowerment: Perceptions of violence among 
urban youth. Journal of Community \& Applied Social Psychology, 17, 147158.

Austin, J., Johnson, K.D., \& Gregoriou, M. (2000). Juveniles in adult prisons and jails: A national assessment. Bureau of Justice Assistance, Office of Justice Programs, U.S. Department of Justice. Retrieved 13 April 2013 from https://www.ncjrs.gov/pdffiles1/bja/182503.pdf.

Ayers, T.S., Sandler, I.N., West, S.G., \& Roosa, M.W. (1996). A dispositional and situational assessment of children's coping: Testing alternative models of coping. Journal of Personality, 64(4), 923-958.

Barnette, J.J. (2000). Effects of stem and Likert response option reversals on survey internal consistency: If you feel the need, there is a better alternative to using those negatively worded stems. Educational and Psychological Measurement, $60(3), 361-370$.

Baumeister, R.F., Bushman, B.J., \& Campbell, K. (2000). Self-esteem, narcissism, and aggression: Does violence result from low self-esteem or from threatened egotism? Current Directions in Psychological Science, 9(1), 26-29.

Bazemore, G. \& Erbe, C. (2003). Operationalizing the community variable in offender reintegration: Theory and practice for developing intervention social capital. Youth Violence and Juvenile Justice, 1, 246-275.

Bazemore, G. \& Terry, W.C. (1997). Developing delinquent youths: A reintegrative model for rehabilitation and a new role for the juvenile justice system. Child Welfare, 76(5), 665-716. 
Bemak, F., Chi-Ying, R., \& Siroskey-Sabdo, L.A. (2005). Empowerment groups for academic success: An innovative approach to prevent high school failure for at-risk, urban African. Professional School of Counseling, 8(5), 377-389.

Benekos, P.J. \& Merlo, A.V. (2008). Juvenile justice: The legacy of punitive policy. Youth Violence and Juvenile Justice, 6(1), 28-46.

Berg, M., Coman, E., \& Schensul, J.J. (2009). Youth action research for prevention: A multi-level intervention designed to increase efficacy and empowerment among urban youth. American Journal of Community Psychology, 43, 345359.

Blumstein, A. (2002). Youth, guns, and violent Crime.

Bobak, M., Pikhart, H., Rose, R., Hertzman, C., \& Marmot, M. (2000).

Socioeconomic factors, material inequalities, and perceived control in selfrated health: Cross-sectional data from seven post-communist countries. Social Science \& Medicine, $51,1343-1350$.

Bullis, M., Yovanoff, P., Mueller, G., \& Havel, E. (2002). Life on the "outs" -Examination of the facility-to-community transition of incarcerated youth. Exceptional Children, 69(1), 7-22.

Bukowski, W.M., Sippola, L., \& Brender, W. (1993). Where does sexuality come from?: Normative sexuality from a development perspective. In H.E. Barbaree, W.L. Marshall, \& S.M. Hudson (Eds.), The Juvenile Sex Offender (pp. 84-103). New York: The Guilford Press. 
Butts, J.A., Mayer, S., \& Ruth, G. (2005). Focus on juvenile justice on positive youth development (Issue brief). Chapin Hall: Center for Children at the University of Chicago. Retrieved 30 March 2012 from http://arachnos.chapinhall.org/sites/default/files/publications/249.pdf.

Butts, J.A. \& Mears, D.P. (2001). Reviving juvenile justice in a get-tough era. Youth \& Society, 33(2), 169-198.

Butts, J.A. \& Travis, J. (2002). The rise and fall of American youth violence: 19802000. Washington, DC: The Urban Institute.

Cargo, M., Grams, G.D., Ottoson, J.M., Ward, P., \& Green, L.W. (2003). Empowerment as fostering positive youth development and citizenship. American Journal of Health Behavior, 27(Suppl. 1), 66-79.

Catalano, R.F., Hawkins, J.D., Berglund, M.L., Pollard, J.A., Arthur, M.W. (2002). Prevention science and positive youth development: Competitive or cooperative frameworks? Journal of Adolescent Health, 31(Supplemental Article), 230-239.

Cattaneo, L.B. \& Chapman, A.R. (2010). The process of empowerment: A model for use in research and practice. American Psychologist, 65(7), 646-659.

Chen, G., Gully, S.M., \& Eden, D. (2004). General self-efficacy and self-esteem: Toward theoretical and empirical distinction between correlated selfevaluations. Journal of Organizational Behavior, 25, 375-395. 
Chinman, M.J. \& Linney, J.A. (1998). Toward a model of adolescent empowerment: Theoretical and empirical evidence. The Journal of Primary Prevention, 18(4), 393-413.

Cleary, T.J. \& Zimmerman, B.J. (2004). Self-regulation empowerment program: A school-based program to enhance self-regulated and self-motivated cycles of student learning. Psychology in the Schools, 41(5), 537-550.

Connor-Smith, J.K., Compas, B.E., Wadsworth, M.E., Thompsen, A.H., \& Saltzman, H. (2000). Journal of Counseling and Clinical Psychology, 68(6), 976-992.

Corcocan, J. (1997). A solution-oriented approach to working with juvenile offenders. Child and Adolescent Social Work Journal, 14(4), 277-288.

Corwyn, R.F. (2000). The factor structure of global self-esteem among adolescents and adults. Journal of Research in Personality, 34(4), 357-379.

Craig, A.R., Franklin, J.A., \& Andrews, G. (1984). A scale to measure locus of control of behavior. British Journal of Medical Psychology, 57, 173-180.

DeSisto, M.C. \& DeSisto, T.P. (2004). School nurses' perceptions of empowerment and autonomy. The Journal of School Nursing, 20(4), 228-233.

Donnellan, M.B., Trzesniewski, K.H. Robins, R.W., Moffitt, T.E., \& Caspi, A. (2005). Low self-esteem is related to aggression, antisocial behavior, and delinquency. Psychological Science, 16(4), 328-335.

Elliott, D.S. \& Ageton, S.S. (1980). Reconciling race and class differences in selfreported and official estimates of delinquency. American Sociological Review, 45(1), 95-110. 
Erikson, E.H. (1968). Identity: Youth and crisis. New York: W.W. Norton \& Company.

Evans, B.K. \& Fischer, D.G. (1992). A hierarchical model of participatory decisionmaking, job autonomy, and perceived control. Human Relations, 45(11), 1169-1189.

Evans, W.D., Ulasevich, A., \& Blahut, S. (2004). Adult and group influences on participation in youth empowerment programs. Health Education \& Behavior, 31(5), 564-576.

Fawcett, S.R., White, G.W., Balcazar, F.E., \& Suarez-Balcazar, Y. (1994). A contextual-behavioral model of empowerment: Case studies involving people with physical disabilities. American Journal of Community Psychology, 22(4), 471-496.

Frans, D.J. (1993). A scale for measuring social worker empowerment. Research and Social Work Practice, 3(3), 312-328.

Freire, P. (1970). Pedagogy of the oppressed. Continuum, New York.

Frick, P. (2002). Juvenile psychopathy from a developmental perspective: Implications for construct development and use in forensic assessments. Law and Human Behavior, 26(2), 247-253.

Fusoni, M. (2005). Teen empowerment: Youth, police, and neighbors in partnership. New Directions for Youth Development, 106, 61-71.

Garcia, T. \& Pintrich, P.R. (1996). The effects of autonomy on motivation and performance in the college classroom. Contemporary Educational 
Psychology, 21, 447-486.

Glick, B. \& Sturgeon, W. (1998). No time to play: Youthful offenders in adult correctional systems. Lanham, MD: American Correctional Association.

Groves, R.M., Fowler, F.J., Couper, M.P., Lepkowski, J.M., Singer, E., \& Tourangeau, R. (2009). Survey methodology ( $2^{\text {nd }}$ Edition). Hoboken, NJ: John Wiley \& Sons, Inc.

Gruber, J. \& Trickett, E. (1987). Can we empower others? The paradox of empowerment in governing an alternative school. American Journal of Community Psychology, 15, 353-371.

Hawkins, J.D., Catalano, R.F., \& Miller, J.Y. (1992). Risk and protective factors for alcohol and other drug problems in adolescence and early adulthood: Implications for substance abuse prevention. Psychological Bulletin, 112(1), 64-105.

Henggeler, S. W., Cunningham, P.B., Pickrel, S.G., Schoenwald, S.K., \& Brondino, M.J. (1996). Multisystemic therapy: An effective violence prevention approach for serious juvenile offenders. Journal of Adolescent, 19, 47-61.

Heppner, P.P. \& Peterson, C.H. (1982). The development and implications of a personal problem-solving inventory. Journal of Counseling Psychology, 29(1), 66-75.

Hinnant, L.W., Nimsch, C., \& Stone-Wiggins, B. (2004). Examination of the relationship between community support and tobacco control activities as a 
part of youth empowerment programs. Health Education \& Behavior, 31(5), 629-640.

Holden, D.J., Crankshaw, E., Nimsch, C., Hinnant, L.W., \& Hund, L. (2004a). Quantifying the impact of participation in local tobacco control groups on the psychological empowerment of involved youth. Health Education \& Behavior, $31(5), 615-628$.

Holden, D.J., Messeri, P., Evans, W.D., Crankshaw, E., Ben-Davies, M. (2004b). Conceptualizing youth empowerment within tobacco control. Health Education \& Behavior, 31(5), 548-563.

Holden, D.J., Evans, W.D., Hinnant, L.W., \& Messeri, P. (2005). Modeling psychological empowerment among youth involved in local tobacco control efforts. Health Education \& Behavior, 32(2), 264-278.

Howell, D. C. (2002). Statistical methods for psychology (5th Edition). Pacific Grove, CA:

Wadsworth.

Hu, L. \& Bentler, P.M. (1999). Cutoff criteria for fit indexes in covariance structure analysis: Conventional criteria versus new alternatives. Structural Equation Modeling, 6(1), 1-55.

Hunter, J. A., Gilbertson, S.A., Vedros, D., \& Morton, M. (2004). Strengthening community-based programming for juvenile sexual offenders: Key concepts and paradigm shifts. Child Maltreatment, 9(2), 177-189. 
Israel, B.A., Checkoway, B., Schulz, A., \& Zimmerman, M. (1994). Health education and community empowerment: Conceptualizing and measuring perceptions of individual, organizational, and community control. Health Education Quarterly, 21(2), 149-170.

Jackson, D.L. (2003). Revisiting sample size and number of parameter estimates: Some support for the $N: q$ hypothesis. Structural Equation Modeling, 10, 128141.

Jennings, L.B., Parra-Medina, D.M., Messias, D.K.H., \& McLoughlin, K. (2006). Toward a critical social theory of youth empowerment. Journal of Community Practice, 14(1/2), 31-55.

Jenson, J.M. \& Howard, M.O. (1998). Youth crime, public policy, and practice in the juvenile justice system: Recent trends and needed reforms. Social Work, 43 (4), 324-334.

John, O.P. \& Benet-Martínez, V. (2000). Measurement: Reliability, construct validity, and scale construction. In H.T. Reis \& C.M. Judd, Handbook of Research: Methods in Social and Personality Psychology (pp. 412-450). New York: Cambridge University Press.

Kaufman, K.L., Hilliker, D.R., Lathrop, P., Daleiden, E.L., \& Rudy, L. (1996). Sexual offenders' modus operandi: A comparison of structured interview and questionnaire approaches. Journal of Interpersonal Violence, 11(1), 19-34. 
Kempf-Leonard, K. (2007). Minority youths and juvenile justice: Disproportionate minority contact after nearly 20 years of reform efforts. Youth Violence and Juvenile Justice, 5(1), 71-87.

Kim, S., Crutchfield, C., Williams, C., \& Hepler, N. (1998). Toward a new paradigm in substance abuse and other problem behavior prevention for youth: Youth development and empowerment approach. Journal of Drug Education, 28(1), $1-17$.

Kline, R.B. (2011). Principles and practice of structural equation modeling ( $3^{\text {rd }}$ edition). New York: The Guilford Press.

Kraimer, M.L., Seibert, S.E., \& Liden, R.C. (1999). Psychological empowerment as a multidimensional construct: A test of construct validity. Educational and Psychological Measurement, 59(1), 127-142.

Krohn, M., Waldo, G.P. Chiricos, T.G. (1974). Self-reported delinquency: A comparison of structured interviews and self-administered checklists. The Journal of Criminal Law \& Criminology, 65(4), 545-553.

Lachman, M.E. \& Weaver, S.L. (1998). The sense of control as a moderator of social class differences in health and well-being. Journal of Personality and Social Psychology, 74(3), 763-773.

Laschinger, H.K.S., Sabiston, J.A., \& Kutszcher, L. (1997). Empowerment and staff nurse decision involvement in nursing work environments: Testing Kanter's theory of structural power in organizations. Research in Nursing \& Health, $20,341-352$. 
Lee, S., Borden, L.M., Serido, J., \& Perkins, D.F. (2009). Ethnic minority youth in youth programs: Feelings of safety, relationships with adult staff, and perceptions of learning skills. Youth \& Society, 41(2), 234-255.

Lerner, R.M., Almerigi, J.B., Theokas, C., \& Lerner, J.V. (2005). Positive youth development: A view of the issues. Journal of Early Adolescence, 25(1), 1016.

LeRoy, L., Benet, D.J., Mason, T., Austin, W.D., Mills, S. (2004). Empowering organizations: Approaches to tobacco control through youth empowerment programs. Health Education \& Behavior, 31(5), 577-596.

Lightfoot, S.L. (1986). On goodness in schools: Themes of empowerment. Peabody Journal of Education, 63(3), 9-28.

Marshall, W.L. (1989). Intimacy, loneliness, and sexual offenders. Behavior Research and Therapy, 27(5), 491-503.

Marshall, W. L., Ward, T., Mann, R.E., Moulden, H., Fernandez, Y.M., Serran, G., \& Marshall, L.E. (2005). Working positively with sexual offenders: Maximizing the effectiveness of treatment. Journal of Interpersonal Violence, 20(9), 10961114.

Maton, K.I. (2008). Empowering community settings: Agents of individual development, community betterment, and positive social change. American Journal of Community Psychology, 41, 4-21. 
Maton, K.I. \& Salem, D.A. (1995). Organizational characteristics of empowering community settings: A multiple case study approach. American Journal of Community Psychology, 23(5), 631-656.

McDonald, R.P. (1999). Test theory: A unified treatment. Mahwah, NJ: Lawrence Erlbaum Associates, Inc.

Menon, S.T. (1999). Psychological empowerment: Definition, measurement, and validation. Canadian Journal of Behavioral Science, 31(3), 161-164.

Messias, D.K.H., Fore, E.M., McLoughlin, K., Parra-Medina, D. (2005). Adult roles in community-based youth empowerment programs: Implications for best practices. Family \& Community Health, 28(4), 320-337.

Mohajer, N. \& Earnest, J. (2009). Youth empowerment for the most vulnerable: A model based on the pedagogy of Freire and experiences in the field. Health Education, 109(5), 424-438.

Moore, K.A. \& Glei, D. (1995). Taking the plunge: An examination of Positive Youth Development. Journal of Adolescent Research, 10(1), 15-40.

Muris, P. (2001). A brief questionnaire for measuring self-efficacy in youths. Journal of Psychopathology and Behavioral Assessment, 23(3), 145-149.

Ng, K.Y., Ang, S., Chan, K.Y. (2008). Personality and leader effectiveness: A moderated mediation model of leadership self-efficacy, job demands, and job autonomy. Journal of Applied Psychology, 93(4), 733-743. 
Office of Juvenile Justice and Delinquency (2011). OJJDP Statistical Briefing Book. Retrieved 10 August 2011 from http://ojjdp.ncjrs.gov/ojstatbb/corrections/qa08201.asp?qaDate=2007.

Office of Juvenile Justice and Delinquency (2011). OJJDP Statistical Briefing Book: Juveniles in Corrections. Retrieved 06 May 2013 from http://www.ojjdp.gov/ojstatbb/corrections/qa08601 .asp?qaDate=2010.

Oregon Youth Authority (2011a). Internal JJIS report. Unpublished.

Oregon Youth Authority (2011b). Affirmative action quarterly statistics charts as of September 30, 2011. Retrieved 19 December 2011 from http://www.oregon.gov/OYA/AA_EEO/AAQuarterlystatistics9302011.pdf.

Oregon Youth Authority (2012a). Annual performance progress report (APPR) for fiscal year (2011-2012). Retrieved 16 April 2013 from http://www.oregon.gov/oya/docs/APPR_final_2012.pdf.

Oregon Youth Authority (2012b). Oregon Youth Authority. Retrieved 25 March 2012 from http://www.oregon.gov/OYA/.

Oregon Youth Authority (2012c). Oregon Youth Authority: Issue Brief: Guiding youth offenders toward positive development. Retrieved 16 April 2013 from http://www.oregon.gov/oya/docs/issue_briefs/ib_pyd.pdf.

Oregon Youth Authority (2013). OYA Quick Facts. Retrieved 24 April 2013 from http://www.oregon.gov/oya/docs/QuickFacts_Jan2013.pdf.

Page, R.M. (1991). Loneliness as a risk factor for adolescent hopelessness. Journal of Research in Personality, 25, 189-195. 
Parker, S.K. \& Ohly, S. (2008). Designing motivating jobs: An expanded framework for linking work characteristics and motivation. In R. Kanfer, G. Chen, \& R. Pritchard (Eds.), Work motivation: Past, present, and future, pp. 233-284. New York: Routledge.

Patrick, A. \& Laschinger, H.K.S. (2006). The effect of structural empowerment and perceived organizational support on middle level nurse managers' satisfaction. Journal of Nursing Management, 14, 13-22.

Patterson, J.M. \& McCubbin, H.I. (1987). Adolescent coping style and behaviors: Conceptualizing and measurement. Journal of Adolescence, 10, 163-186.

Paulhus, D. (1983). Sphere-specific measures of perceived control. Journal of Personality and Social Psychology, 44(6), 1253-1265.

Pearson, L.C. \& Moomaw, W. (2005). The relationship between teacher autonomy and stress, satisfaction, empowerment, and professionalism. Educational Research Quarterly, 29(1), 37-53.

Perkins, D.D. \& Zimmerman, M.A. (1995). Empowerment theory, research, and application. American Journal of Community Psychology, 23(5), 569-579.

Peters, C.S. \& Myrick, S. (2011). Juvenile recidivism - measuring success or failure: Is there a difference? Corrections Today, 73(1), 41-44.

Peterson, A.C. (1988). Adolescent development. Annual Review of Psychology, 39, 583-607.

Peterson, N.A., Lowe, J.B., Hughey, J., Reid, R.J., Zimmerman, M.A., \& Speer, P.W. (2006). Measuring the intrapersonal component of psychological 
empowerment: Confirmatory factor analysis of the sociopolitical control scale. American Journal of Community Psychology, 38, 287-297.

Pittman, K.J., Irby, M., Tolman, J., Yohalem, N., \& Ferber, T. (2003). Preventing problems, promoting development, encouraging engagement: Competing priorities or inseparable goals? The Forum for Youth Investment, Impact Strategies, Inc. Retreived 30 March 2011 from http://casel.org/wpcontent/uploads/2011/04/Preventing-Problems-Promoting-DevelopmentEncouraging-Engagement.pdf.

Puzzanchera, C., Adams, B. \& Kang, W. (2012). Easy access to FBI arrest statistics: 1994-2009. Office of Juvenile Justice and Delinquency Prevention. Retrieved 14 Rappaport, J. (1981). In praise of paradox: A social policy of empowerment over prevention. American Journal of Community Psychology, 9, 14-25.

Rappaport, J. (1987). Terms of Empowerment/Exemplars of Prevention: Toward a Theory for Community Psychology. American Journal of Community Psychology.

Rappaport, J. (1998). The art of social change: Community narratives as resources for individual and collective identity. In X.B. Arriaga \& S. Oskamp (Eds.), Addressing community problems: Psychological research and interventions, pp. 225-246. Thousand Oaks, CA: Sage.

Riger, S. (1993). What's wrong with empowerment? American Journal of Community Psychology, 21, 279-292. 
Ribisl, K.M., Steckler, A., Linnan, L., Patterson, C.C., Pevzner, E.S., Markatos, E., Goldstein, A.O., McGloin, T., \& Peterson, A.B. (2004). The North Carolina youth empowerment study (NC YES): A participatory research study examining the impact of youth empowerment for tobacco use prevention. Health Education \& Behavior, 31(5), 597-614.

Roth, J.L. \& Brooks-Gunn, J. (2003). What exactly is a youth development program? Answers from research and practice. Applied Developmental Science, 7(2), 94-111.

Rotter, J.B (1966). Generalized expectancies for internal versus external control of enforcement. Psychological Monographs, 80(1), 1-28.

Rudkin, J.K. (2003). Community psychology: Guiding principles and orienting concepts. Upper Saddle River, NJ: Pearson Education.

Scherer, M, Maddux, J.E., Mercandante, B., Prentice-Dunn, S., Jacobs, B., Rogers, R.W. (1982). The Self-Efficacy Scale: Construction and validation. Psychological Reports, 51, 663-671.

Schmitz, M.F. \& Baer, J.C. (2001). The vicissitudes of measurement: A confirmatory factor analysis of the emotional autonomy scale. Child Development, 72(1), 207-219.

Schwartz, R.G. (2000). Juvenile justice and positive youth development. In Youth Development: Issues, Challenges, and Directions, pp. 233-280. Philadelphia: Public/Private Ventures.

Schwarzer, R., Babler, J., Kwiatek, P., Schroder, K. (1997). The assessment of 
optimistic self-beliefs: Comparison of the German, Spanish, and Chinese versions of the general self-efficacy scale. Applied Psychology: An International Review, 46(1), 69-88.

Schwarzer, R., Mueller, J., \& Greenglass, E. (1999). Assessment of perceived general self-efficacy on the internet: Data collection in cyberspace. Anxiety, Stress, \& Coping, 12, 145-161.

Seagrave, D. \& Grisso, T. (2002). Adolescent development and the measurement of juvenile psychopathy. Law and Human Behavior, 26(2), 219-239.

Seibert, S.E., Silver, S.R., \& Randolph, W.A. (2004). Taking empowerment to next level: A multiple-level model of empowerment, performance, and satisfaction. Academy of Management Journal, 47(3), 332-349.

Sickmund, M., Snyder, H.N., \& Poe-Yamagata, E. (1997). Juvenile offenders and victims: 1997 update on violence. Office of Juvenile Justice and Deliquency Prevention, Office of Justice Programs, U.S. Department of Justice. Retrieved 9 April 2012 from https://www-ncjrsgov.proxy.lib.pdx.edu/pdffiles/juvoff.pdf.

Snyder, H.N. \& Sickmund, M. (2006). Juvenile offenders and victims: 2006 national report. Office of Juvenile Justice and Deliquency Prevention, Office of Justice Programs, U.S. Department of Justice. Retrieved 9 April 2012 from http://www.ojjdp.gov/ojstatbb/nr2006/downloads/NR2006.pdf. 
Speer, P.W. \& Peterson, A. (2000). Psychometric properties of an empowerment scale: Testing cognitive, emotional, and behavioral domain. Social Work Research, 24(2), 109-118.

Spencer, M.B. \& Jones-Walker, C. (2004). Interventions and services offered to former juvenile offenders reentering their communities: An analysis of program effectiveness. Youth Violence and Juvenile Justice, 2(1), 88-97.

Spittal, M., Siegert, R., McClure, J., \& Walkey, F. (2002). The spheres of control scale: The identification of a clear replicable three-factor structure. Personality and Individual Differences, 32, 121-131.

Steinberg, L. (2002). Adolescence. San Francisco: McGraw Hill. Steinberg, L., Chung, H.L., Little, M. (2004). Reenty of young offenders from the justice system: A developmental perspective. Youth Violence and Juvenile Justice, 2(1), 21-38.

Steinberg, L. \& Morris, A.S. Adolescent development. Annual Review of Psychology, $52,83-110$.

Tabachnick, B.G. \& Fidell, L.S. (2007). Using Multivariate Statistics (5 ${ }^{\text {th }}$ Edition). San Francisco: Pearson Education, Inc.

van der Helm, P., Stams, G.J., \& van der Laan, P. (2011). Measuring group climate in prison. The Prison Journal, 91(2), 158-176.

Wallerstein, N. (1992). Powerlessness, empowerment, and health: Implication for health promotion programs. American Journal of Health Promotion, 6(3), 197-205. 
Wallerstein, N. \& Bernstein, E. (1988). Empowerment education: Freire's ideas adapted to health education. Health Education \& Behavior, 15(4)(379-394).

Wang, G. \& Netemeyer, R.G. (2002). The effects of job autonomy, customer demandingness, and trait competitiveness on salesperson, learning, selfefficacy, and performance. Journal of Academy of Marketing Science, 30(3), 217-228.

Ward, T. \& Mann, R. (2004). Good lives and the rehabilitation of offenders: A positive approach to sex offender treatment. In P.A. Linley and S. Joseph (Ed.), Positive psychology in practice. Hoboken, NJ: Wiley \& Sons, Inc.

Ward, T. \& Stewart, C.A. (2003). The treatment of sex offenders: Risk management and good lives. Professional Psychology: Research and Practice, 34(4), 353360.

Wegener, D.T. \& Fabrigar, L.E. (2000). Analysis and design for nonexperimental data: Addressing causal and noncausal hypotheses. In H.T. Reis \& C.M. Judd, Handbook of Research: Methods in Social and Personality Psychology (pp. 412-450). New York: Cambridge University Press.

Williams, G.C., Grow, V.M., Freedman, Z.R., Ryan, R.M., \& Deci, E.L. (1996). Motivational predictors of weight loss and weight-loss maintenance. Journal of Personality and Social Psychology, 70(1), 115-126.

Wilson, N., Minkler, M., Dasho, S., Wallerstein, M, \& Martin, A.C. (2008). Getting to social action: The Youth Empowerment Strategies (YES!) project. Health Promotion Practice, 9(4), 395-403. 
Wormith, J. S., Althouse, R., Simpson, M., Reitzel, L.R., Fagan, T.J., \& Morgan, R.D. (2007). The rehabilitation and reintegration of offenders: The current landscape and some future directions for correctional psychology. Criminal Justice and Behavior, 34(7), 879-892.

Wright, K.N. (1985). Developing the Prison Environment Inventory. Journal of Research in Crime and Delinquency, 22(3), 257-277.

Yuen, J.W.L. \& Shaughnessy, B. (2001). Cultural empowerment: Tools to engage and retain postsecondary students with disabilities. Journal of Vocational Rehabilitation, 16, 199-207.

Zeldin, S. (2004). Integrating research and practice to understand and strengthen communities for adolescent development: An introduction to the special issue and current issues. Applied Development Science, 4(Supplemental 1), 2-10.

Zeldin, S. (2004). Preventing youth violence through the promotion of community engagement and membership. Journal of Community Psychology, 32(5), 623641.

Zeldin, S. \& Price, L.A. (1995). Creating supportive communities for adolescent development: Challenges to scholars: An introduction. Journal of Adolescent Research, 10(1), 6-14.

Zimmerman, M.A. (1990). Toward a theory of learned hopefulness: A structural model analysis of participation and empowerment. Journal of Research in Personality, 24, 71-86. 
Zimmerman, M.A. (1995). Psychological empowerment: Issues and Illustrations. American Journal of Community Psychology, 23(5), 581-599.

Zimmerman, M.A., Israel, B., Schulz, A., \& Checkoway, B. (1992). Further explorations in empowerment theory: An empirical analysis of psychological empowerment. American Journal of Community Psychology, 20(6), 707-727.

Zimmerman, M.A. \& Rappaport, J. (1988). Citizen participation, perceived control, and psychological empowerment. American Journal of Community Psychology, 16(5), 725-750.

Zimmerman, M.A. \& Warschauski, S. (1998). Empowerment theory for rehabilitation research: Conceptual and methodological issues. Rehabilitation Psychology, 43(1), 3-16.

Zimmerman, M.A. \& Zahniser, J.H. (1991). Refinements of sphere-specific measures of perceived control: Development of a sociopolitical control scale. Journal of Community Psychology, 19, 189-204. 
APPENDIX A: OYA Annual Survey.

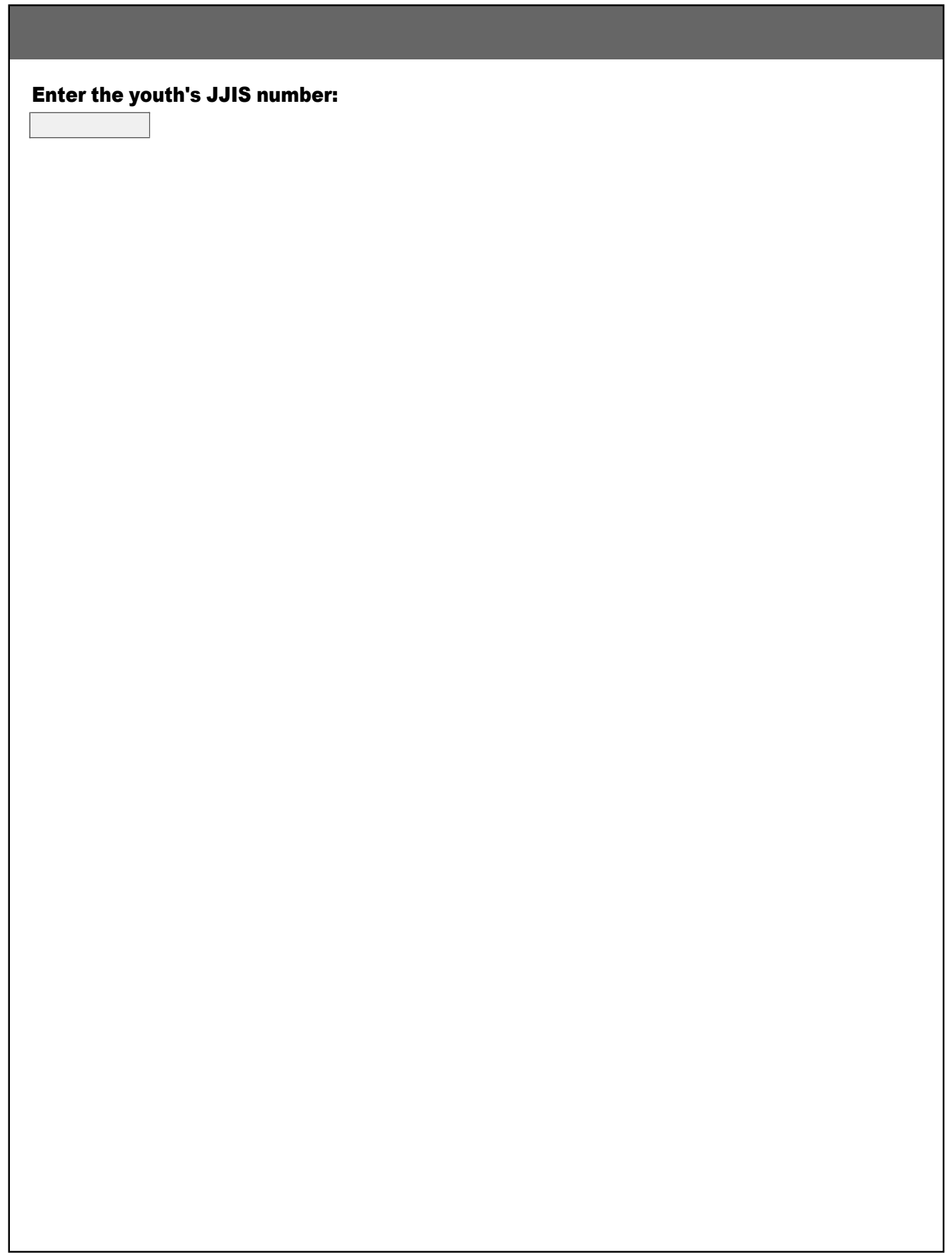


You are invited to take part in a survey for youth who are living at OYA close custody facilities. This survey will ask you a lot of different questions about your facility, treatment, and staff. There will also be questions about recreation and exercise at your facility.

If you were living at an OYA facility last summer, you probably took a survey a lot like this. This survey is being done by the OYA Research and Evaluation Unit. THIS IS NOT THE PBS SURVEY.

All of your answers and anything you tell us in this survey will be KEPT SECRET. Only the researchers will know that you took the survey. When we talk about the results of the survey to other people, we will never talk about you specifically. We will only talk about the results as a group. Please be honest.

Please read the directions at the top of each page before answering the questions. If you have a question, please raise your hand. 
The statements below are about living units. We want to know how you feel about YOUR living unit. Think about the unit where you are living and say how much you agree or disagree with each of the statements below. There are no right or wrong answers. We just want your opinion. All of your answers will be kep secret. Please be honest.

\begin{tabular}{|c|c|c|c|c|c|}
\hline & $\begin{array}{c}\text { Strongly } \\
\text { Agree }\end{array}$ & Agree & Neutral & Disagree & $\begin{array}{l}\text { Strongly } \\
\text { Disagree }\end{array}$ \\
\hline I keep busy by participating in activities. & 0 & C & C & 0 & 0 \\
\hline Staff tell me when I do well. & 0 & 0 & $\mathrm{O}$ & $\mathrm{O}$ & $\mathrm{O}$ \\
\hline I exercise when I want to. & 6 & C & C & 0 & O \\
\hline I feel anxious when I am on my living unit. & 0 & 0 & 0 & 0 & 0 \\
\hline I am working on my future here. & 0 & 0 & 0 & 0 & 0 \\
\hline
\end{tabular}

Please choose the best answer to the following statements.

\begin{tabular}{|c|c|c|c|c|c|}
\hline & $\begin{array}{l}\text { Strongly } \\
\text { Agree }\end{array}$ & Agree & Neutral & Disagree & $\begin{array}{l}\text { Strongly } \\
\text { Disagree }\end{array}$ \\
\hline The unit is quiet. & 0 & 0 & 0 & 0 & 0 \\
\hline I enjoy interacting with staff. & 0 & 0 & 0 & 0 & 0 \\
\hline I know the rules. & 0 & 0 & 0 & 0 & 0 \\
\hline I have to ask for permission for everything. & 0 & 0 & 0 & 0 & 0 \\
\hline $\begin{array}{l}\text { If I let other people know I do not want to be bothered, other people will } \\
\text { not bother me. }\end{array}$ & 0 & 0 & 0 & 0 & 0 \\
\hline
\end{tabular}

Please choose the best answer to the following statements.

I learn new skills here.

I am allowed to read or watch TV when I want.

Strongly
Agree
0
0
0
0

$\begin{array}{cccc}\text { Agree } & \text { Neutral } & \text { Disagree } & \begin{array}{c}\text { Strongly } \\ \text { Disagree }\end{array} \\ 0 & 0 & 0 & 0 \\ 0 & 0 & 0 & 0 \\ 0 & 0 & 0 & 0 \\ 0 & 0 & 0 & 0 \\ 0 & 0 & 0 & 0\end{array}$

Please choose the best answer to the following statements.

Staff allow me space if I need it.

I know what my goals are.

$\begin{array}{ccccc}\begin{array}{c}\text { Strongly } \\ \text { Agree }\end{array} & \text { Agree } & \text { Neutral } & \text { Disagree } & \begin{array}{c}\text { Strongly } \\ \text { Disagree }\end{array} \\ 0 & 0 & 0 & 0 & 0 \\ 0 & 0 & 0 & 0 & 0 \\ 0 & 0 & 0 & 0 & 0 \\ 0 & 0 & 0 & 0 & 0 \\ 0 & 0 & 0 & 0 & 0\end{array}$

I let my peers know I care about them.

What I am learning here will help me when I leave. 


\begin{tabular}{|c|c|c|c|c|c|}
\hline \multicolumn{6}{|c|}{ Please choose the best answer to the following statements. } \\
\hline & $\begin{array}{l}\text { Strongly } \\
\text { Agree }\end{array}$ & Agree & Neutral & Disagree & $\begin{array}{l}\text { Strongly } \\
\text { Disagree }\end{array}$ \\
\hline If I try something new, staff encourage me. & o & 0 & 0 & 0 & 0 \\
\hline I can be alone without being disturbed. & 0 & 0 & 0 & 0 & 0 \\
\hline If I feel anxious, I have someone to talk to. & 0 & 0 & 0 & 0 & 0 \\
\hline These surroundings make me feel depressed. & 0 & 0 & 0 & 0 & 0 \\
\hline I know what will happen if I violate the rules. & 0 & 0 & 0 & 0 & 0 \\
\hline \multicolumn{6}{|c|}{ Please choose the best answer to the following statements. } \\
\hline & $\begin{array}{l}\text { Strongly } \\
\text { Agree }\end{array}$ & Agree & Neutral & Disagree & $\begin{array}{l}\text { Strongly } \\
\text { Disagree }\end{array}$ \\
\hline I feel comfortable here. & o & 0 & 0 & 0 & 0 \\
\hline I can read without being disturbed. & 0 & 0 & 0 & 0 & 0 \\
\hline Staff help me with my problems. & 0 & 0 & 0 & 0 & 0 \\
\hline I care about other residents. & 0 & 0 & 0 & 0 & 0 \\
\hline I do not have to work if I do not want to. & 0 & 0 & 0 & 0 & 0 \\
\hline \multicolumn{6}{|c|}{ Please choose the best answer to the following questions. } \\
\hline & $\begin{array}{l}\text { Strongly } \\
\text { Agree }\end{array}$ & Agree & Neutral & Disagree & $\begin{array}{l}\text { Strongly } \\
\text { Disagree }\end{array}$ \\
\hline What I am learning here is helping me. & 0 & 0 & 0 & 0 & 0 \\
\hline I have something to do every night. & 0 & 0 & 0 & 0 & 0 \\
\hline Staff tease me if I am depressed. & 0 & 0 & 0 & 0 & 0 \\
\hline I know what will get me written up by staff. & 0 & 0 & 0 & 0 & 0 \\
\hline I know when the canteen is open. & 0 & 0 & 0 & 0 & 0 \\
\hline \multicolumn{6}{|c|}{ Please choose the best answer to the following statements. } \\
\hline & $\begin{array}{l}\text { Strongly } \\
\text { Agree }\end{array}$ & Agree & Neutral & Disagree & $\begin{array}{l}\text { Strongly } \\
\text { Disagree }\end{array}$ \\
\hline Staff don't have enough time for me. & 0 & 0 & 0 & c & 0 \\
\hline I am learning the right things here. & 0 & 0 & 0 & 0 & 0 \\
\hline $\begin{array}{l}\text { I am willing to go up and talk to other residents even if they are } \\
\text { strangers. }\end{array}$ & 0 & 0 & 0 & 0 & 0 \\
\hline I can obtain vocational training if I want. & 0 & 0 & 0 & 0 & 0 \\
\hline I am making progress here. & 0 & 0 & 0 & 0 & 0 \\
\hline
\end{tabular}




\begin{tabular}{|c|c|c|c|c|c|}
\hline \multicolumn{6}{|c|}{ Please choose the best answer to the following statements. } \\
\hline & $\begin{array}{l}\text { Strongly } \\
\text { Agree }\end{array}$ & Agree & Neutral & Disagree & $\begin{array}{l}\text { Strongly } \\
\text { Disagree }\end{array}$ \\
\hline I spend several hours each day talking with peers. & 0 & 0 & 0 & 0 & 0 \\
\hline $\begin{array}{l}\text { Treatment programs will help me make parole. (If you are a DOC youth } \\
\text { you may skip this question) }\end{array}$ & 0 & 0 & ○ & 0 & 0 \\
\hline I feel that staff respect me for my ethnicity. & 0 & 0 & 0 & 0 & 0 \\
\hline I feel that other residents respect me for my ethnicity. & 0 & 0 & 0 & 0 & ○ \\
\hline I feel that staff respect me for my gender. & 0 & 0 & 0 & 0 & 0 \\
\hline I feel that other residents respect me for my gender. & 0 & 0 & 0 & 0 & ○ \\
\hline I feel that staff respect me for my sexual orientation. & 0 & 0 & 0 & 0 & 0 \\
\hline I feel that other residents respect me for my sexual orientation. & 0 & 0 & 0 & 0 & 0 \\
\hline
\end{tabular}


The sentences below talk about beliefs about yourself in your LIVING UNIT at this facility. When reading each sentence, think about what you did and how you felt IN THE LAST MONTH.

\section{IN MY LIVING UNIT...}

\begin{tabular}{|c|c|c|c|c|c|}
\hline & $\begin{array}{c}\text { Strongly } \\
\text { Agree }\end{array}$ & Agree & Neutral & Disagree & $\begin{array}{l}\text { Strongly } \\
\text { Disagree }\end{array}$ \\
\hline If something looks too complicated, I will not even bother to try it. & $\mathrm{C}$ & 0 & 0 & 0 & 0 \\
\hline In general, I would prefer to be a leader rather than a follower. & O & $\mathrm{O}$ & O & 0 & O \\
\hline I can influence decisions that are made. & 0 & 0 & 0 & 0 & 0 \\
\hline $\begin{array}{l}\text { I am unsure about where to go if I have a major problem with an OYA } \\
\text { staff member, like he or she makes fun of me and it hurts my feelings. }\end{array}$ & O & $\mathrm{O}$ & $\mathrm{O}$ & $\mathrm{O}$ & O \\
\hline The beliefs of other youth at OYA make it difficult to do what is right. & 0 & 0 & 0 & 0 & C \\
\hline $\begin{array}{l}\text { When making a decision, I weigh the consequences of each choice and } \\
\text { compare them against each other. }\end{array}$ & 0 & 0 & 0 & 0 & 0 \\
\hline $\begin{array}{l}\text { I let things go rather than to speak up about them, even if they are } \\
\text { important to me. }\end{array}$ & C & C & C & 0 & O \\
\hline I actively participate in activities, even if I don't have to. & 0 & 0 & 0 & 0 & 0 \\
\hline I only have myself to rely on for support. & 0 & 0 & 0 & 0 & 0 \\
\hline I am confident that I can work effectively on many different tasks. & 0 & 0 & 0 & 0 & 0 \\
\hline When I work on group projects, I prefer to "take a back seat." & 0 & 0 & 0 & 0 & 0 \\
\hline $\begin{array}{l}\text { There is little I can do to change many of the things that are important } \\
\text { to me. }\end{array}$ & 0 & 0 & 0 & 0 & 0 \\
\hline $\begin{array}{l}\text { I know where to go to get information about starting a new activity like a } \\
\text { basketball tournament. }\end{array}$ & 0 & 0 & 0 & 0 & 0 \\
\hline OYA staff have little influence on my behavior. & 0 & 0 & 0 & 0 & 0 \\
\hline $\begin{array}{l}\text { When confronted with a problem, I tend to do the first thing that I can } \\
\text { think of to solve it. }\end{array}$ & 0 & 0 & 0 & 0 & 0 \\
\hline I speak up about issues that are important to me. & 0 & 0 & 0 & 0 & 0 \\
\hline $\begin{array}{l}\text { If given the choice, I prefer to do other things rather than participate in } \\
\text { activities. }\end{array}$ & 0 & 0 & 0 & 0 & 0 \\
\hline There is at least one other youth I can go to for support. & 0 & 0 & 0 & 0 & 0 \\
\hline
\end{tabular}


The sentences below talk about beliefs about yourself in your TREATMENT GROUP at this facility. When reading each sentence, think about what you did and how you felt IN THE LAST MONTH.

\section{IN MY TREATMENT GROUP...}

When I work on group projects, I prefer to "take a back seat."

There is little I can do to change many of the things that are important to me.

I know where to go to get information about my progress on my treatment goals.

OYA staff have little influence on my behavior.

$\begin{array}{ccccc}\begin{array}{c}\text { Strongly } \\ \text { Agree }\end{array} & \text { Agree } & \text { Neutral } & \text { Disagree } & \begin{array}{c}\text { Strongly } \\ \text { Disagree }\end{array} \\ 0 & 0 & 0 & 0 & 0 \\ 0 & 0 & 0 & 0 & 0 \\ 0 & 0 & 0 & 0 & 0 \\ 0 & 0 & 0 & 0 & 0\end{array}$

When confronted with a problem, I tend to do the first thing that I can think of to solve it.

I speak up about issues that are important to me.

If given the choice, I prefer to do other things rather than participate in activities.

If something looks to complicated, I will not even bother to try it.

In general, I would prefer to be a leader rather than a follower.

I only have myself to rely on for support.

I can influence decisions that are made.

I am unsure about where to go if I have a major problem with an OYA staff member, like I disagree with something she or he asked me to work on.

The beliefs of other youth at OYA make it difficult to do what is right.

When making a decision, I weigh the consequences of each choice and compare them against each other.

I let things go rather than to speak up about them, even if they are important to me.

I actively participate in activities, even if I don't have to. 6

c

0

0

There is at least one other youth I can go to for support.

\section{C}

C

C

C $C$

C $\mathrm{C}$

C 0

C 6

C $c$

$0 \quad 0$

o 0

C $\quad 0$

C $C$

$6 \quad 6$

o 0

0

0

○

0

$$
0
$$

o

0

$0 \quad 0$

00

0

00

00

00

o

$\begin{array}{lll}0 & 0 & 0 \\ 0 & 0 & 0 \\ 0 & 0 & 0\end{array}$

\section{o}

6

$\begin{array}{ll}0 & 0 \\ 0 & 0\end{array}$


The sentences below talk about beliefs about yourself in your SCHOOL or VOCATIONAL TRAINING at this facility. When reading each sentence, think about what you did and how you felt IN THE LAST MONTH.

IN SCHOOL Or VOCATIONAL TRAINING...

I am unsure about where to go if I have a major problem with an OYA staff member, like he or she says mean or rude things about my work.

I can influence decisions that are made.

When I work on group projects, I prefer to "take a back seat."

If something looks too complicated, I will not even bother to try it.

When making a decision, I weigh the consequences of each choice and

\begin{tabular}{|c|c|}
\hline $\begin{array}{l}\text { Strongly } \\
\text { Agree }\end{array}$ & Agree \\
\hline 0 & 0 \\
\hline 0 & 0 \\
\hline 0 & 0 \\
\hline 0 & 0 \\
\hline 0 & 0 \\
\hline 0 & 0 \\
\hline
\end{tabular}

compare them against each other.

I let things go rather than to speak up about them, even if they are important to me.

If given the choice, I prefer to do other things rather than participate in activities.

There is at least one other youth I can go to for support.

OYA staff have little influence on my behavior.

I know where to go to get information about taking classes outside of OYA.

There is little I can do to change many of the things that are important to me.

I am confident that I can work effectively on many different tasks.

In general, I would prefer to be a leader rather than a follower.

When confronted with a problem, I tend to do the first thing that I can think of to solve it.

I speak up about issues that are important to me.

I actively participate in activities, even if I don't have to

I only have myself to rely on for support.

$\begin{array}{lllll}0 & 0 & 0 & 0 & 0 \\ 0 & 0 & 0 & 0 & 0 \\ 0 & 0 & 0 & 0 & 0 \\ 0 & 0 & 0 & 0 & 0 \\ 0 & 0 & 0 & 0 & 0 \\ 0 & 0 & 0 & 0 & 0 \\ 0 & 0 & 0 & 0 & 0 \\ 0 & 0 & 0 & 0 & 0 \\ 0 & 0 & 0 & 0 & 0 \\ 0 & 0 & 0 & 0 & 0 \\ 0 & 0 & 0 & 0 & 0 \\ 0 & 0 & 0 & 0 & 0\end{array}$


These items are related to you and your facility. Facilities have different styles of dealing with youth and we would like to know more about how you feel about your experiences. Your responses will be kept secret. Please be honest.

I feel that my facility gives me a reasonable amount of choices and
options.
I feel understood by staff.
I am able to be open with staff.
Staff show confidence in my ability to make changes.
I feel that staff accept me.
Staff make sure that I understand my issues and what I need to do.


The following questions ask you about the treatment you have participated in while at OYA. Your responses will be kept secret. Please be honest.

The treatment I receive is helpful.
I feel I have changed because of treatment.
I think the treatment programs are boring.
The staff who lead treatment are good at it.
I wish treatment was different than it is.
I have a hard time relating to the treatment books.
I learn things in treatment.
Staff don't seem to like the treatment programs.
The youth in my treatment groups don't pay attention.
I think about things differently because of treatment.
I like the treatment programs I have participated in.
I look forward to treatment groups.
I think I am getting the right kind of treatment for me.


The following questions ask you about recreation/exercise you have participated in while at OYA. Your responses will be kept secret. Please be honest.

\begin{tabular}{|c|c|c|c|c|c|}
\hline & $\begin{array}{c}\text { Strongly } \\
\text { Agree }\end{array}$ & Agree & Neutral & Disagree & $\begin{array}{l}\text { Strongly } \\
\text { Disagree }\end{array}$ \\
\hline I feel like I get enough exercise. & 0 & 0 & 0 & 0 & 0 \\
\hline Having more time for exercise would be good for me. & 0 & 0 & 0 & 0 & 0 \\
\hline I always know when my unit will have recreation/exercise time. & 0 & 0 & 0 & 0 & 0 \\
\hline Recreation/Exercise time never gets canceled. & $\mathrm{O}$ & 0 & 0 & 0 & 0 \\
\hline There are lots of activities to choose from during recreation/exercise. & 0 & 6 & 0 & 0 & 0 \\
\hline Staff help organize activities for youth. & $\mathrm{O}$ & 0 & 0 & 0 & 0 \\
\hline I would feel better if I had more opportunities for exercise. & 0 & 0 & 0 & 0 & 0 \\
\hline Gym time is very important to me. & $\mathrm{O}$ & 0 & 0 & 0 & 0 \\
\hline If recreation/exercise is canceled, it is usually for a good reason. & 0 & 6 & 0 & $\mathrm{O}$ & 0 \\
\hline Exercise helps me with my mood. & 0 & 0 & 0 & 0 & 0 \\
\hline
\end{tabular}


Have you ever participated in the Hope

Partnership program?

(The Hope Partnership is a program that is only offered at MacLaren YCF.)

0 Yes

C No 
Tell us what you think about the Hope Partnership program.

Strongly agree

The Hope Partnership program is helping me become

better.
Agree

$O$

Neutral

○

Disagree

Strongly disagree 
This last set of questions is a chance for you to look at how you think and feel about yourself. Your responses will be kept secret. Please be honest.

I can make changes now that will help me avoid problems in the future.
A lot of what happens in my life is because of bad luck.
I can only control my problems if other people help me.
When I make plans, I can always make them work.
The problems I have now will stay with me all my life.
People's lives are determined by things outside of their control.
Luck or chance mostly determine my future.
Its my job to deal with my problems and mistakes, no one else's.
If I work hard, I will be successful--luck won't have anything to do with it.
My life is controlled by outside actions and events.
I need professional help to manage my problems.
When I am stressed out, there isn't much I can do to calm myself down.
I think it's up to each person to determine their own future.
I understand what my problems are.
I know that I will be able to deal with problems in the future.
The only reason I can keep my problems under control is because I'm

lucky. 
Thank you so much for taking this survey!

Please take a second to tell us anything else you think we should know. Remember, everything you tell us on this survey will be kept secret. 


\section{APPENDIX B: Treatment Manager Survey on Behavioral Success in Five Domains.}

Today's Date

Youth OYA ID

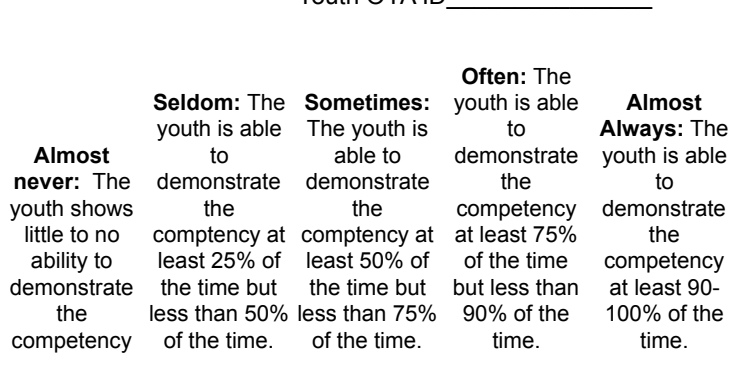

Directions: The following statements are indicators of competency in five different domains. Please use the rating scale below to indicate how often the youth demonstrates each competency.

\begin{tabular}{|c|c|c|c|c|c|c|}
\hline & EDUCATION DOMAIN & $\begin{array}{l}\text { Almost } \\
\text { Never }\end{array}$ & Seldom & Sometimes & Often & $\begin{array}{l}\text { Almost } \\
\text { Always }\end{array}$ \\
\hline 1 & $\begin{array}{l}\text { Demonstrates involvement in school } \\
\text { activities. }\end{array}$ & 1 & 2 & 3 & 4 & 5 \\
\hline 2 & Exhibits a willing to learn. & 1 & 2 & 3 & 4 & 5 \\
\hline 3 & $\begin{array}{l}\text { Plans education path to meet personal long } \\
\text { term goals. }\end{array}$ & 1 & 2 & 3 & 4 & 5 \\
\hline \multirow[t]{2}{*}{4} & $\begin{array}{l}\text { Routinely discusses education process with } \\
\text { teachers. }\end{array}$ & 1 & 2 & 3 & 4 & 5 \\
\hline & LIFE/SOCIAL SKILLS DOMAIN & $\begin{array}{l}\text { Almost } \\
\text { Never }\end{array}$ & Seldom & Sometimes & Often & $\begin{array}{l}\text { Almost } \\
\text { Always }\end{array}$ \\
\hline 1 & $\begin{array}{l}\text { Demonstrates ability to pro-socially engage } \\
\text { others. }\end{array}$ & 1 & 2 & 3 & 4 & 5 \\
\hline 2 & Displays appropriate social ettiquette. & 1 & 2 & 3 & 4 & 5 \\
\hline 3 & $\begin{array}{l}\text { Exhibits ability to decide whether to join in } \\
\text { with others. }\end{array}$ & 1 & 2 & 3 & 4 & 5 \\
\hline 4 & Initiates social time with positive peers. & 1 & 2 & 3 & 4 & 5 \\
\hline \multirow[t]{2}{*}{5} & $\begin{array}{l}\text { Resolves conflict in a manner appropriate to } \\
\text { situation. }\end{array}$ & 1 & 2 & 3 & 4 & 5 \\
\hline & MENTAL HEALTH DOMAIN & $\begin{array}{l}\text { Almost } \\
\text { Never }\end{array}$ & Seldom & Sometimes & Often & $\begin{array}{l}\text { Almost } \\
\text { Always }\end{array}$ \\
\hline 1 & $\begin{array}{l}\text { Demonstrates ability to monitor/manage } \\
\text { symptoms. }\end{array}$ & 1 & 2 & 3 & 4 & 5 \\
\hline 2 & $\begin{array}{l}\text { Demonstrates good coping skills with normal } \\
\text { life stressors. }\end{array}$ & 1 & 2 & 3 & 4 & 5 \\
\hline 3 & $\begin{array}{l}\text { Describes plan of action when symptoms } \\
\text { appear. }\end{array}$ & 1 & 2 & 3 & 4 & 5 \\
\hline 4 & Develops alternative coping skills. & 1 & 2 & 3 & 4 & 5 \\
\hline 5 & Develops stress management skills. & 1 & 2 & 3 & 4 & 5 \\
\hline
\end{tabular}




\begin{tabular}{|c|c|c|c|c|c|c|}
\hline & OFFENSE SPECIFIC DOMAIN & $\begin{array}{l}\text { Almost } \\
\text { Never }\end{array}$ & Seldom & Sometimes & Often & $\begin{array}{l}\text { Almost } \\
\text { Always }\end{array}$ \\
\hline 1 & $\begin{array}{l}\text { Avoids physical, verbal, and relational } \\
\text { violence. }\end{array}$ & 1 & 2 & 3 & 4 & 5 \\
\hline 2 & Exhibits motivation to change. & 1 & 2 & 3 & 4 & 5 \\
\hline 3 & $\begin{array}{l}\text { Identifies impact on self, family, victims, and } \\
\text { community. }\end{array}$ & 1 & 2 & 3 & 4 & 5 \\
\hline 4 & $\begin{array}{l}\text { Understands logical consequences of violent } \\
\text { behavior. }\end{array}$ & 1 & 2 & 3 & 4 & 5 \\
\hline \multirow[t]{2}{*}{5} & $\begin{array}{l}\text { Utilizes peer resistance to avoid dangerous } \\
\text { situations. }\end{array}$ & 1 & 2 & 3 & 4 & 5 \\
\hline & VOCATION DOMAIN & $\begin{array}{l}\text { Almost } \\
\text { Never }\end{array}$ & Seldom & Sometimes & Often & $\begin{array}{l}\text { Almost } \\
\text { Always }\end{array}$ \\
\hline 1 & $\begin{array}{l}\text { Exhibits positive work relationship with } \\
\text { supervisor. }\end{array}$ & 1 & 2 & 3 & 4 & 5 \\
\hline 2 & $\begin{array}{l}\text { Exhibits positive work relationship with co- } \\
\text { workers. }\end{array}$ & 1 & 2 & 3 & 4 & 5 \\
\hline 3 & $\begin{array}{l}\text { Explores vocational skills necessary to } \\
\text { pursue employment. }\end{array}$ & 1 & 2 & 3 & 4 & 5 \\
\hline 4 & $\begin{array}{l}\text { Pursues available training to meet vocational } \\
\text { interests. }\end{array}$ & 1 & 2 & 3 & 4 & 5 \\
\hline
\end{tabular}


APPENDIX C: Additional Validation Check of Intrapersonal PE.

\section{Rationale}

Although not originally planned as part of this study, it was recognized that additional analyses could be conducted to further explore the validity of the Intrapersonal PE scale. Support for construct validity could be enhanced through the examination of Intrapersonal PE based on youth demographic differences across OYA facilities (i.e., criterion-group validity). For example, given the knowledge that youth gain more complex cognitive abilities (e.g., critical thinking, abstract thinking, advanced reasoning) associated with psychological empowerment as they age (Steinberg, 2002), it would be possible to test for differences in PE across OYA facilities based on the mean age of the population that they serve. Demographic variables investigated included age, ethnicity, commitment disposition (i.e., OYA or DOC), and total time incarcerated.

Results in the predicted direction would indicate that higher psychological empowerment scores are more likely to be reported in some facilities due, in part, to the demographic features of its youth. Where no differences are anticipated, nonsignificant findings would also lend credence to construct validity.

Procedure

Initial analyses provided statistics for each of the targeted demographic variables. Analyses were conducted to assess between-group facility population differences on each of the demographic variables. If the factor analysis of the 
Psychological Empowerment scale supported its utility in this study, ANOVAs could have been utilized to compare PE scores across OYA facilities.

Results

Demographic Variables and Between Facility Differences. In order to better understand the sample of incarcerated young men, demographic information was explored. Specifically, age, ethnicity, commitment disposition (i.e., OYA or DOC), and total time incarcerated were assessed. Two one-way ANOVAs and two chisquare tests of independence were conducted to assess demographic differences between facilities.

One-way ANOVAs were conducted to investigate differences in age and total time incarcerated between facilities. There was a significant difference in current age of participants within the nine facilities, $F(8,526)=26.08, p<.001$, partial $\eta^{2}=.28$. Levene's test indicated that the homogeneity of variance assumption was not satisfied, $F(8,526)=2.87, p=.004$. Post hoc tests that accounted for differences in variances between groups (i.e., Games-Howell) indicated that there were significant differences in age between several facilities. Refer to Appendix C Table 1 for results of these paired comparisons.

There also were significant differences in total time incarcerated between the nine facilities, $F(8,526)=20.01, p<.001$, partial $\eta^{2}=.23$. The assumption for homogeneity of variance was not satisfied, $F(8,526)=6.04, p<.001$; therefore, post hoc tests were conducted using Games-Howell pairwise comparisons (see Appendix C Table 2). Significant differences in age and total time incarcerated between the nine 
facilities were to be expected as facilities house youth at different points during their sentence. Therefore, it was not surprising that, for example, youth at CF2 were significantly younger than youth at all three transitional facilities. CF2 typically houses youth during the intake process and latter three facilities are specific to the transition-to-community process, which generally involves older youth.

Two chi-square tests of independence were conducted to examine differences in ethnicity and commitment disposition (i.e., OYA or DOC) between facilities. While significant differences between facilities existed, $\chi^{2}(40)=76.45, p<.001$, Cramer's $\mathrm{V}=.17$ and $\chi^{2}(8)=79.17, p<.001$, Cramer's $\mathrm{V}=.39$, respectively, these differences were again to be expected, particularly for commitment disposition. As previously mentioned, facilities house youth at different points in time during their sentence and provide different services; therefore, it is not surprising to see, for example, that the large majority $(84.80 \%)$ of youth residing at North Coast were sentenced within OYA. North Coast provides substance abuse treatment, and while OYA and DOC youth might equally struggle with substance abuse issues, DOC youth have been convicted of more serious crimes under Measure 11 and thus typically receive different treatment services. Additionally, significant differences in ethnicity between facilities might also be due, in part, to low cell counts for ethnic minorities. Refer to Appendix C Table 3 for descriptive differences in commitment disposition and ethnicity between facilities.

Confirmatory Analyses on the Psychological Empowerment Scale. Factor analyses did not support the use of the psychological empowerment scale. The only 
sub-scale that was found to fit the data well was Intrapersonal PE in the living unit, treatment groups, and school or vocational activities. Even though the single-factor structure was supported for Intrapersonal PE, its internal consistency was low in all three settings. Thus, further empirical work is needed in order to improve the operationalization of this construct. Additional analyses to explore aspects of construct validity of Intrapersonal PE, and psychological empowerment more broadly, were not warranted because revisions to the sub-scale are required. Construct validation through the aforementioned analyses would be useful after the scale has consistently been supported statistically.

Since the factor analysis of the Psychological Empowerment scale was not psychometrically sound, it did not make sense to further explore its validity. If future work on this scale has a more positive result, it would be possible to use comparisons of this nature to explore the validity of such a scale. Findings from these analyses are the first steps toward establishing construct validity based on group differences. 


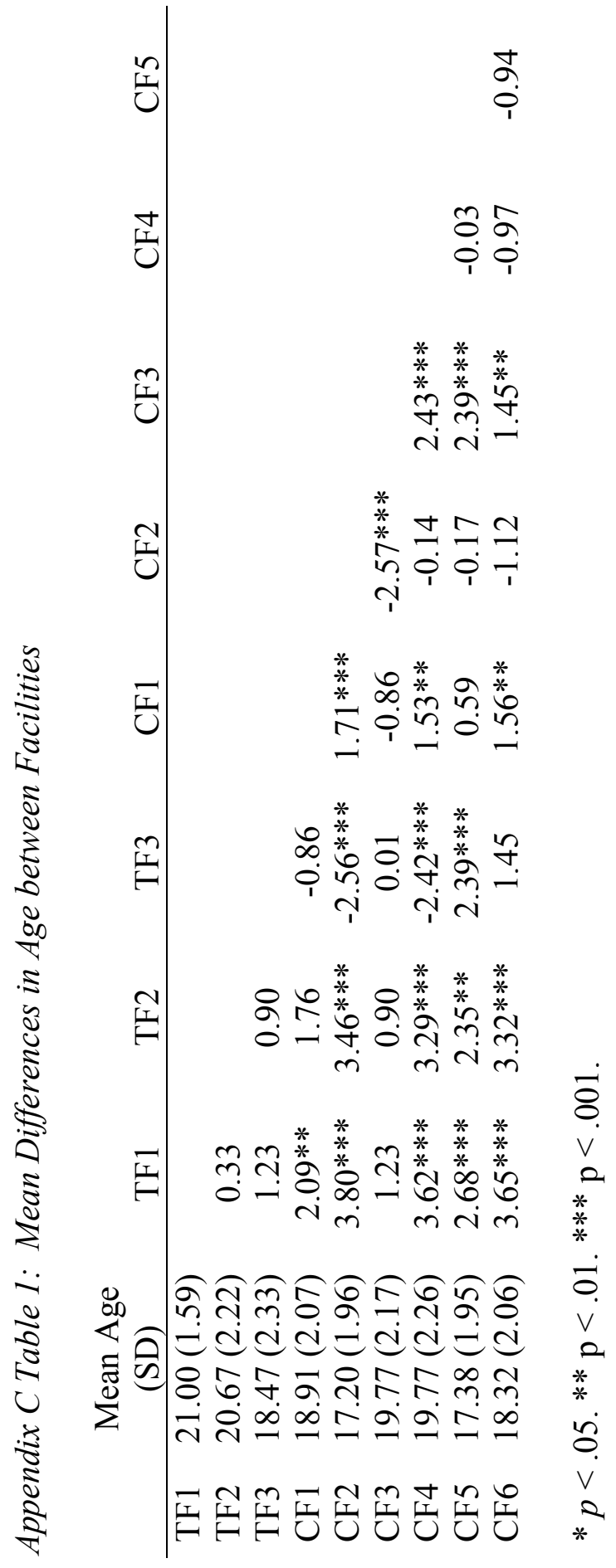




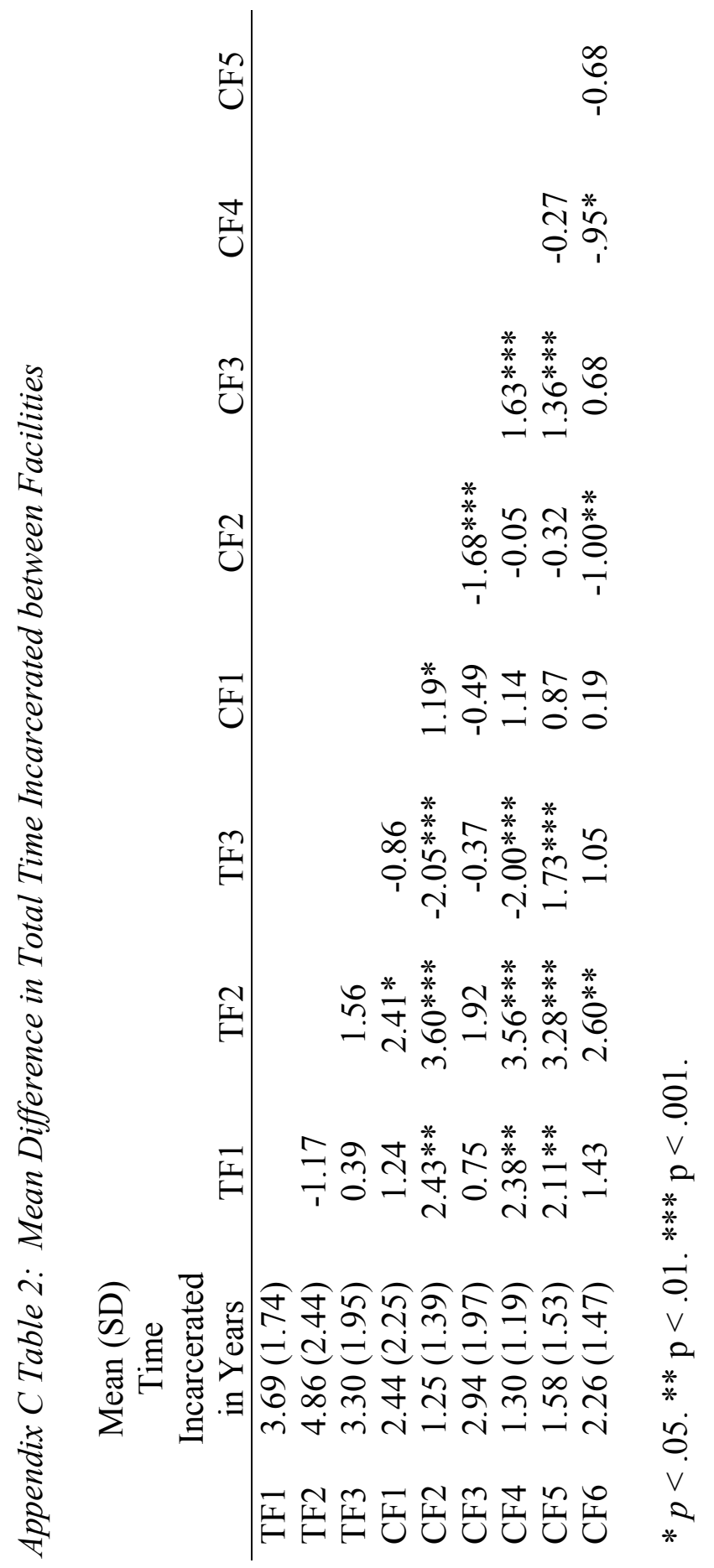




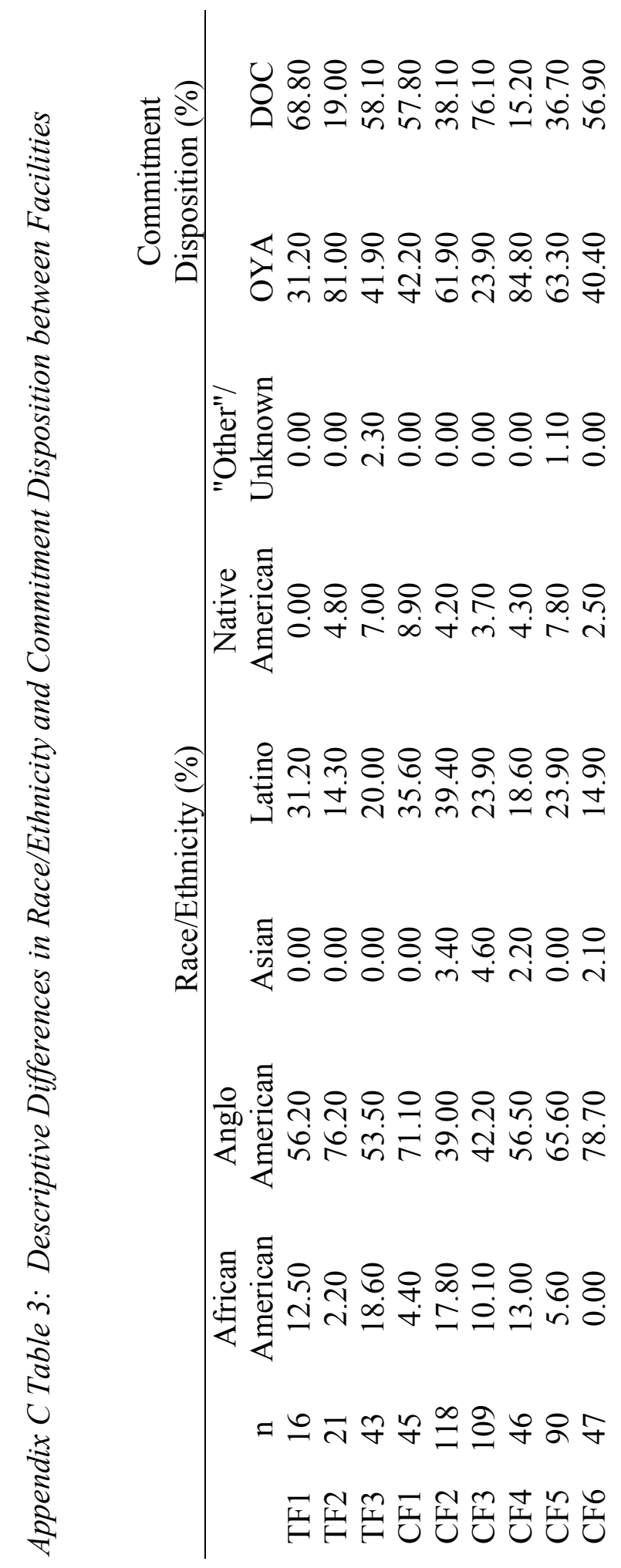

